\title{
AN ASSESSMENT OF NONPOINT-SOURCE DISCHARGES, STREAMFLOW, AND WATER QUALITY IN ONION RIVER, WISCONSIN
}

By

S.J. Field and R.A. Lidwin

U.S. GEOLOGICAL SURVEY

Water-Resources Investigations

Report 84-4066

Prepared in cooperation with the

Wisconsin Department of Natural Resources

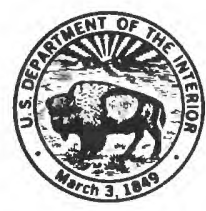

August, 1984 


\title{
UNITED STATES DEPARTMENT OF THE INTERIOR
}

\author{
WILLIAM P. CLARK. SECRETARY
}

\section{GEOLOGICAL SURVEY}

Dallas L. Peck, Director

For additional information write to:

District Chief

U.S. Geological Survey, WRD

1815 University Avenue

Madison, Wisconsin 53705-4096
Copies of this report can be purchased from:

Open-File Services Section

Western Distribution Branch

U.S. Geological Survey

Box 25425, Federal Center

Denver, Colorado $\mathbf{8 0 2 2 5}$

(Telephone: (303) 2341-5888) 


\section{CONTENTS}

Page

Abstract $\ldots \ldots \ldots \ldots \ldots \ldots \ldots \ldots \ldots \ldots \ldots \ldots \ldots \ldots \ldots \ldots \ldots \ldots \ldots \ldots$

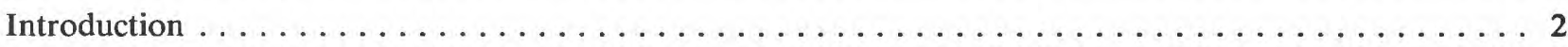

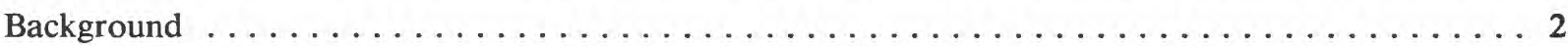

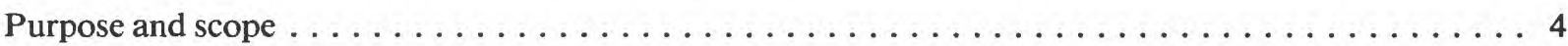

Acknowledgments . . . . . . . . . . . . . . . . . . . . . . . . 4

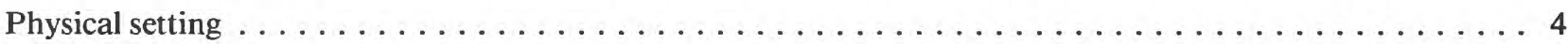

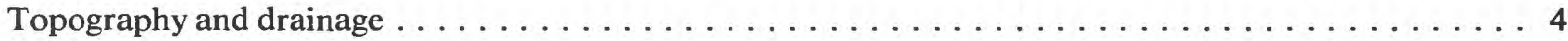

Stream-channel characteristics . . . . . . . . . . . . . . . . . . . . 4

Climate. . . . . . . . . . . . . . . . . . . . . . . . . . 4

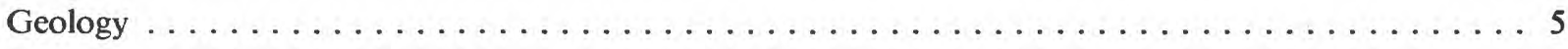

Soils . . . . . . . . . . . . . . 5

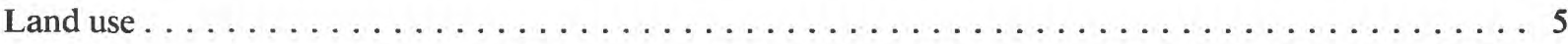

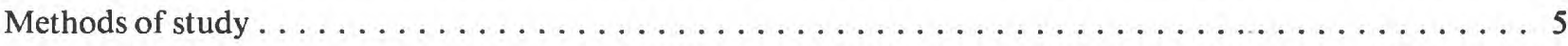

Streamflow $\ldots \ldots \ldots \ldots \ldots \ldots \ldots \ldots \ldots \ldots \ldots \ldots \ldots \ldots \ldots \ldots \ldots \ldots \ldots \ldots$

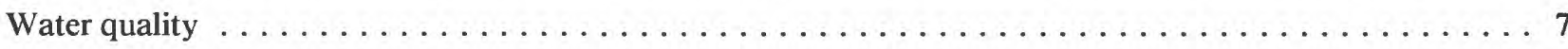

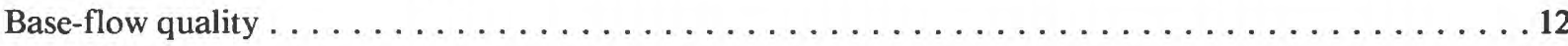

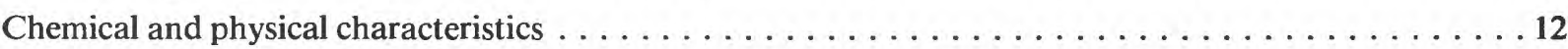

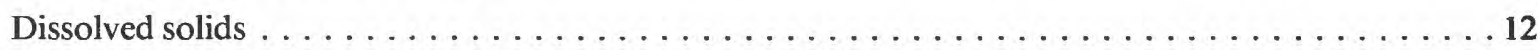

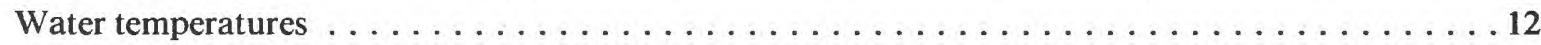

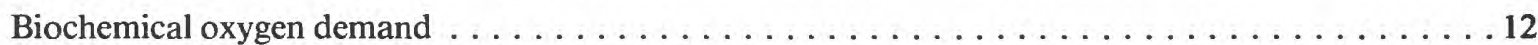

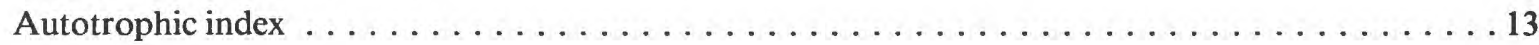

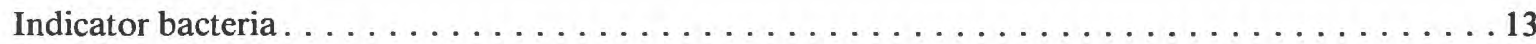

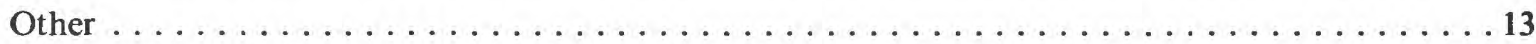

Relation of suspended sediment and nutrients to streamflow $\ldots \ldots \ldots \ldots \ldots \ldots \ldots \ldots \ldots \ldots \ldots$

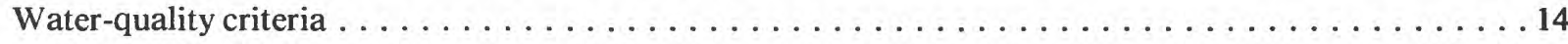

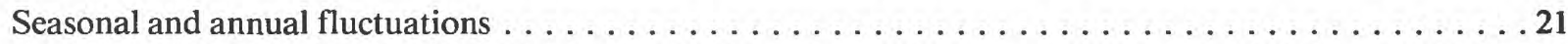

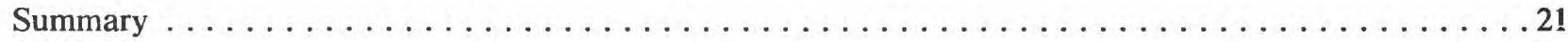

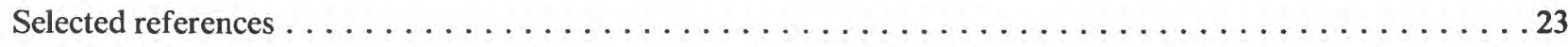




\section{ILLUSTRATIONS}

Page

Figure 1. Map showing location of the Onion River basin in Wisconsin $\ldots \ldots \ldots \ldots \ldots \ldots$

2. Graph showing double mass-accumulation curve of monthly runoff for Onion River at Hingham and Onion River downstream of Hingham $\ldots \ldots \ldots \ldots \ldots$

3. Hydrographs of base flow and surface runoff for Onion River at Hingham,

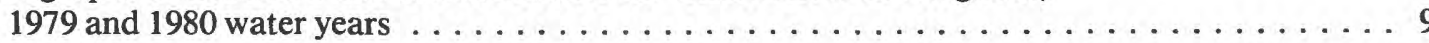

4. Hydrographs of base flow and surface runoff for Onion River near Sheboygan

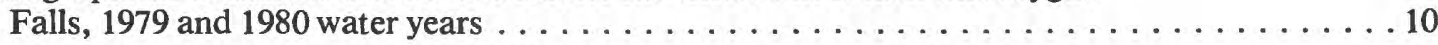

5. Total phosphorus concentration trends of dry- and wet-weather surveys

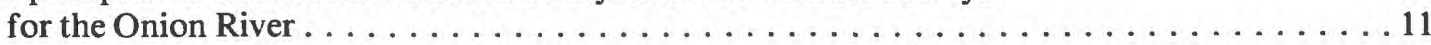

6-12. Graphs showing:

6. Double mass-accumulation curves of suspended sediment as a function of monthly runoff for Onion River at Hingham and Onion River downstream of Hingham . . 15

7. Double mass-accumulation curves of phosphorus and orthophosphate phosphorus as a function of monthly runoff for Onion River at Hingham . . . . . . . . . 16

8. Double mass-accumulation curves of phosphorus and orthophosphate phosphorus as a function of monthly runoff for Onion River downstream of Hingham . . . . . . . 17

9. Double mass-accumulation curves of total nitrogen, ammonia nitrogen, nitrite plus nitrate nitrogen, and organic nitrogen as a function of monthly runoff for Onion River at Hingham. . . . . . . . . . . . . . . . 18

10. Double mass-accumulation curves of total nitrogen, ammonia nitrogen, nitrite plus nitrate nitrogen, and organic nitrogen as a function of monthly runoff for Onion River downstream of Hingham . . . . . . . . . . . . . . . . . . 19

11. Double mass-accumulation curves of dissolved solids as a function of monthly runoff for Onion River at Hingham and Onion River downstream of Hingham . . . . . . . 20

12. Average monthly suspended-sediment and total phosphorus concentrations during low flow in the Onion River and Steiner Branch $\ldots \ldots \ldots \ldots \ldots \ldots \ldots \ldots \ldots \ldots$

\section{TABLES}

Table 1. Percentage of land use in the Onion River basin $\ldots \ldots \ldots \ldots \ldots \ldots \ldots \ldots \ldots \ldots \ldots \ldots \ldots$

2. Summary of streamflow characteristics for Onion River at Hingham, Onion River near Sheboygan Falls, and Sheboygan River at Sheboygan, 1979 and 1980 water years . . . . 25

3. Precipitation and runoff for Onion River near Sheboygan Falls for selected periods . . . . . . . 25

4. Base flow and storm runoff for Onion River at Hingham and Onion River at Sheboygan Falls, 1979 and 1980 water years . . . . . . . . . . . . . . . 26

5. Comparison of base-flow discharges at Hingham, Gibbsville, and Sheboygan Falls . . . . . . 26

6. Concentrations of nutrients in base-flow samples from the Onion River at

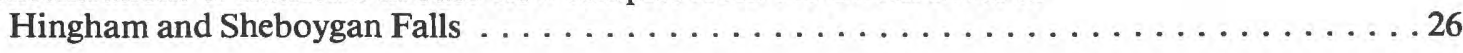

7. Constituent loads and percentage of annual load for storms sampled $\ldots \ldots \ldots \ldots \ldots \ldots \ldots$

8. Loads and yields of chemical constituents in the Onion River basin,

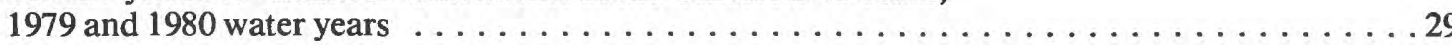


9. Suspended-sediment and nutrient concentrations during low-flow and storm-runoff periods in the Onion River, Steiner Branch, and Bruce Valley Creek . . . . . . . . . . . . 29

10. Stream discharges for Onion River at Hingham, 1979 and 1980 water years . . . . . . . . 30

11. Stream discharges for Onion River near Sheboygan Falls, 1979 and 1980 water years . . . . . . . . . . . . . . . . . . . . 31

12. Water and bed-material analyses from Onion River at Hingham, 1979 and

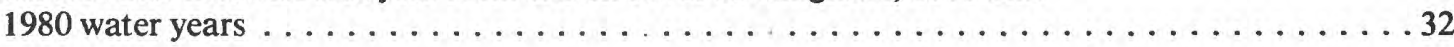

13. Water and bed-material analyses from Onion River near Sheboygan Falls,

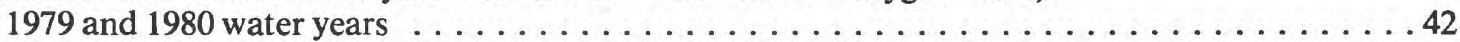

14. Water temperatures for Onion River at Hingham, 1979 and 1980 water years . . . . . . . . 53

15. Water temperatures for Onion River near Sheboygan Falls, 1979 and 1980 water years . . . . . . . . . . . . . . . . . . 56

16. Suspended-sediment loads for Onion River at Hingham, 1979 and $1980 \ldots \ldots \ldots \ldots \ldots$

17. Suspended-sediment loads for Onion River near Sheboygan Falls, 1979

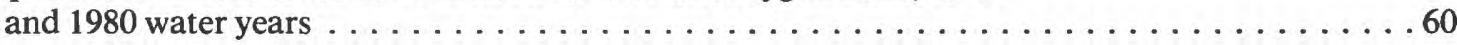

18. Total organic nitrogen load, in pounds per day, for Onion River at Hingham, 1979 and 1980 water years . . . . . . . . . . . . . . . . . . . 61

19. Total nitrogen load, in pounds per day, for Onion River at Hingham,

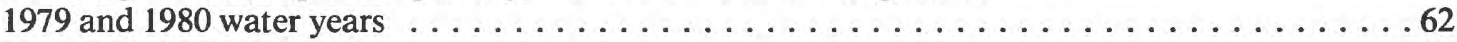

20. Total nitrite plus nitrate nitrogen load, in pounds per day, for Onion River at Hingham, 1979 and 1980 water years . . . . . . . . . . . . . 63

21. Total ammonia nitrogen load, in pounds per day, for Onion River at Hingham, 1979 and 1980 water years . . . . . . . . . . . . . . . . . . . 64

22. Total phosphorus load, in pounds per day, for Onion River at Hingham,

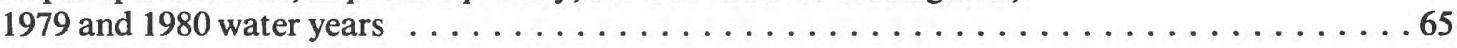

23. Total orthophosphate phosphorus load, in pounds per day, for Onion River

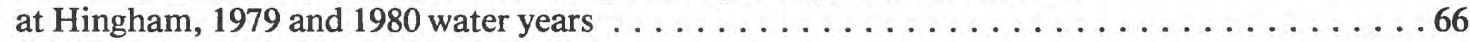

24. Total organic nitrogen load, in pounds per day, for Onion River at Hingham, 1979 and 1980 water years . . . . . . . . . . . . . . . . . 67

25. Total nitrogen load, in pounds per day, for Onion River at Hingham,

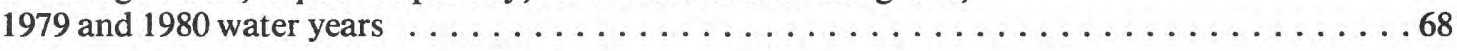

26. Total nitrite plus nitrate nitrogen load, in pounds per day, for Onion River near Sheboygan Falls, 1979 and 1980 water years

27. Total ammonia nitrogen load, in pounds per day, for Onion River near

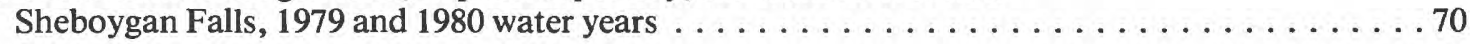

28. Total phosphorus load, in pounds per day, for Onion River near Sheboygan

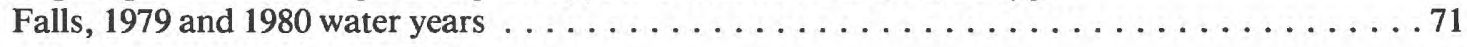

29. Total orthophosphate phosphorus load, in pounds per day, for Onion River near Sheboygan Falls, 1979 and 1980 water years . . . . . . . . . . . . . . 72

30. Specific conductance for Onion River at Hingham, 1979 and 1980 water years . . . . . . . 73

31. Specific conductance for Onion River near Sheboygan Falls, 1979 and 1980 water years . . . . . . . . . . . . . . . . $6 \ldots \ldots \ldots \ldots \ldots \ldots \ldots$ 


\section{CONVERSION TABLE}

For readers who prefer to use International System of Units (SI) rather than inch-pound units, conversion factors for terms used in this report are listed below. Multiply inch-pound unit By

inch (in.)

foot (ft)

mile (mi)

square mile $\left(\mathrm{mi}^{2}\right)$

foot per mile ( $\mathrm{ft} / \mathrm{mi})$

pound per square mile $\left(\mathrm{lb} / \mathrm{mi}^{2}\right)$

ton per square mile (ton $\left./ \mathrm{mi}^{2}\right)$

cubic foot per second $\left.\left(\mathrm{ft}^{3}\right) / \mathrm{s}\right)$

degrees Fahrenheit $\left({ }^{\circ} \mathrm{F}\right)$
25.40

0.3048

1.609

2.590

0.1894

$1.751 \times 10^{2}$

0.3503

$2.832 \times 10^{2}$

$0.555(\mathrm{~F}-32)$ To obtain SI unit

millimeter (mm)

meter $(m)$

kilometer $(\mathrm{km})$

square kilometer $\left(\mathrm{km}^{2}\right)$

meter per kilometer $(\mathrm{m} / \mathrm{km})$

kilogram per square kilometer $\left(\mathrm{kg} / \mathrm{km}^{2}\right)$

metric ton per square $\left(\mathrm{t} / \mathrm{km}^{2}\right)$

cubic meter per second $\left(\mathrm{m}^{3} / \mathrm{s}\right)$

degrees Celsius $\left({ }^{\circ} \mathrm{C}\right)$ 


\title{
AN ASSESSMENT OF NONPOINT-SOURCE DISCHARGES, STREAMFLOW, AND WATER QUALITY IN ONION RIVER, WISCONSIN
}

\author{
By
}

\author{
S.J. Field and R.A. Lidwin
}

\begin{abstract}
The Onion River in eastern Wisconsin was studied during the 1979 and 1980 water years to define the relationship between water quality and streamflow. Agricultural nonpoint-source discharges in the lower part of the Onion River are suspected of contributing significantly to degradation of water quality.

Two streamflow water-quality monitoring stations were established on the Onion River, one at Hingham upstream of the affected area, and one near Sheboygan Falls downstream of the affected area. Streamflow at Onion River at Hingham ranged from a minimum 7-day mean low flow $\left(\mathrm{Q}_{7}\right)$ of 8.7 to a maximum discharge of 600 cubic feet per second; at Onion River near Sheboygan Falls streamflow ranged from a $Q_{7}$ of 13 to a maximum discharge of 2,350 cubic feet per second. Based on discharges at the Sheboygan River at Sheboygan, these discharges ranged from about double the low flow that occurs on the average of once every 2 years, to discharges of between 5-and 10-year recurrence intervals. The average discharges at Hingham in the 1979 and 1980 water were 32.2 and 27.6 cubic feet per second, respectively. At Sheboygan Falls, the average discharges in the 1979 and 1980 water years were 94.2 and 55.2 cubic feet per second, respectively. Based on the average discharge at the Sheboygan River at Sheboygan, the 1979 discharge was 60 percent greater than average, and the 1980 discharge was about 5 percent less than average.
\end{abstract}

Precipitation in the study area for the 1979 water year was 33.1 inches and for the 1980 water year it was 36.8 inches; these amounts are 3.3 inches and 7.0 inches, respectively, more than average.

Suspended-sediment yields and, probably, phosphorus yields were slightly above average for both years because of greater than normal precipitation, and stream discharges that were greater than normal in 1979 and near normal in 1980. Suspended-sediment yields were 79.1 tons per square mile for the 1979 water year and 63.9 tons per square mile for the 1980 water year at Hingham, while downstream of Hingham the yields were 93.5 tons per square mile for the 1979 water year and 84.2 tons per square mile for the 1980 water year. Phosphorus yields were 331 pounds per square mile for the 1979 water year and 317 pounds per square mile for the 1980 water year at Hingham. Downstream of Hingham, the phosphorus yields were 656 pounds per square mile for the 1979 water year and 647 pounds per square mile for the 1980 water year.

A population of bottom-dwelling carp resuspends the bottom sediments during its late spring and early summer active period, possibly causing high concentrations of suspended sediment and phosphorus.

Nutrient yields and loading rates were highest downstream of Hingham. Nonpoint-source contribution of phosphorus amounted to 362 pounds per square mile downstream of Hingham compared to 
272 pounds per square mile upstream of Hingham in the 1979 water year.

Part of the high nutrient yields are due to point sources from Belgium Creek, which drains an area of 16.2 square miles. In the 1979 water year, point sources contributed an estimated 16,700 pounds of phosphorus or 45 percent of the total annual load downstream of Hingham, whereas, upstream of Hingham point sources contributed an estimated 2,200 pounds of phosphorus, or 18 percent of the total annual load.

At Hingham during base flow, four of five phosphorus concentrations exceeded levels recommended by the U.S. Environmental Protection Agency; most concentrations during storm runoff exceeded these levels. At Sheboygan Falls, all phosphorus concentrations at base flow and most storm runoff concentrations also exceeded the Environmental Protection Agency criteria.

All samples at both Hingham and Sheboygan Falls contained concentrations of un-ionized ammonia that were less than the U.S. Environmental Protection Agency's criteria ( 0.02 milligrams per liter), except the March 1980 samples. No samples exceeded the Wisconsin Department of Natural Resources criteria for un-ionized ammonia (0.04 milligrams per liter).

\section{INTRODUCTION}

\section{Background}

In 1972, Congress mandated, through the Federal Water Pollution Control Act Amendments (FWPCAA) ${ }^{1}$ that the surface waters of the United States shall be "fishable and swimmable" by 1983 (U.S. Congress, 1972). To reach this goal, the states must identify and establish programs to improve water quality. It was evident that water-quality goals established by the FWPCAA of 1972 cannot be attained by regulation of only point-source pollution. Indeed, in many areas pollutants discharged from nonpoint sources constitute the major contribution to water-quality degradation (Donigon and Crawford, 1976).

The Wisconsin Department of Natural Resources (DNR) has been designated as the State agency responsible for water-quality protection in Wisconsin (Wisconsin Department of Natural Re- sources, 1976) and has a primary role in meeting Section 208 requirements. However, to assess nonpoint-source effects on surface-water quality, a data base must first be established. To assist in establishing an adequate data base, the U.S. Geological Survey in cooperation with DNR, began a study in 1977 to define the water quality in relation to streamflow in several areas where surface-water quality has been degraded by nonpoint sources.

Among the river basins studied were the Steiner Branch basin in Lafayette County in southwest Wisconsin and the Elk Creek basin (including Bruce Valley Creek) in Trempealeau County in westcentral Wisconsin.

In 1978 the Wisconsin Legislature enacted the Wisconsin Nonpoint Source Water Pollution Abatement Program (Wisconsin Fund). The program works through "priority watersheds" to provide cost sharing and technical assistance to individual property owners, cities, and villages for the control of nonpoint sources of water pollution. To be eligible for cost sharing and technical assistance under the Wisconsin Fund the area must be designated as a priority watershed. The watersheds are selected through a three-step process involving an impartially ranked list of watersheds, regional advisory groups, and the State Nonpoint Coordinating Committee. The first of nine priority watersheds throughout the State to be selected was the Onion River in eastern Wisconsin (Wisconsin Department of Natural Resources, 1981).

This report describes the study done in the Onion River basin in Sheboygan and Ozaukee Counties. Ninety percent of the Onion River basin is in Sheboygan County. The study area is located about $10 \mathrm{mi}$ southwest of Sheboygan (fig. 1). Agricultural nonpoint-source runoff was identified as a major contribution to degraded water quality (Wisconsin Department of Natural Resources, 1979). In DNR's preliminary assessment of the basin in 1978, they found that the waters of the Onion River upstream of Waldo had water quality capable of supporting trout. However, downstream of the Waldo-Hingham area the water quality degraded rapidly. They also found that between Waldo and Gibbsville a large carp population reduced water clarity by resuspending silt and clay deposits.

The nutrient loads in this report reflect the contribution from point and nonpoint sources. Although this report will deal primarily with nonpoint sources, point sources will be discussed brief- 


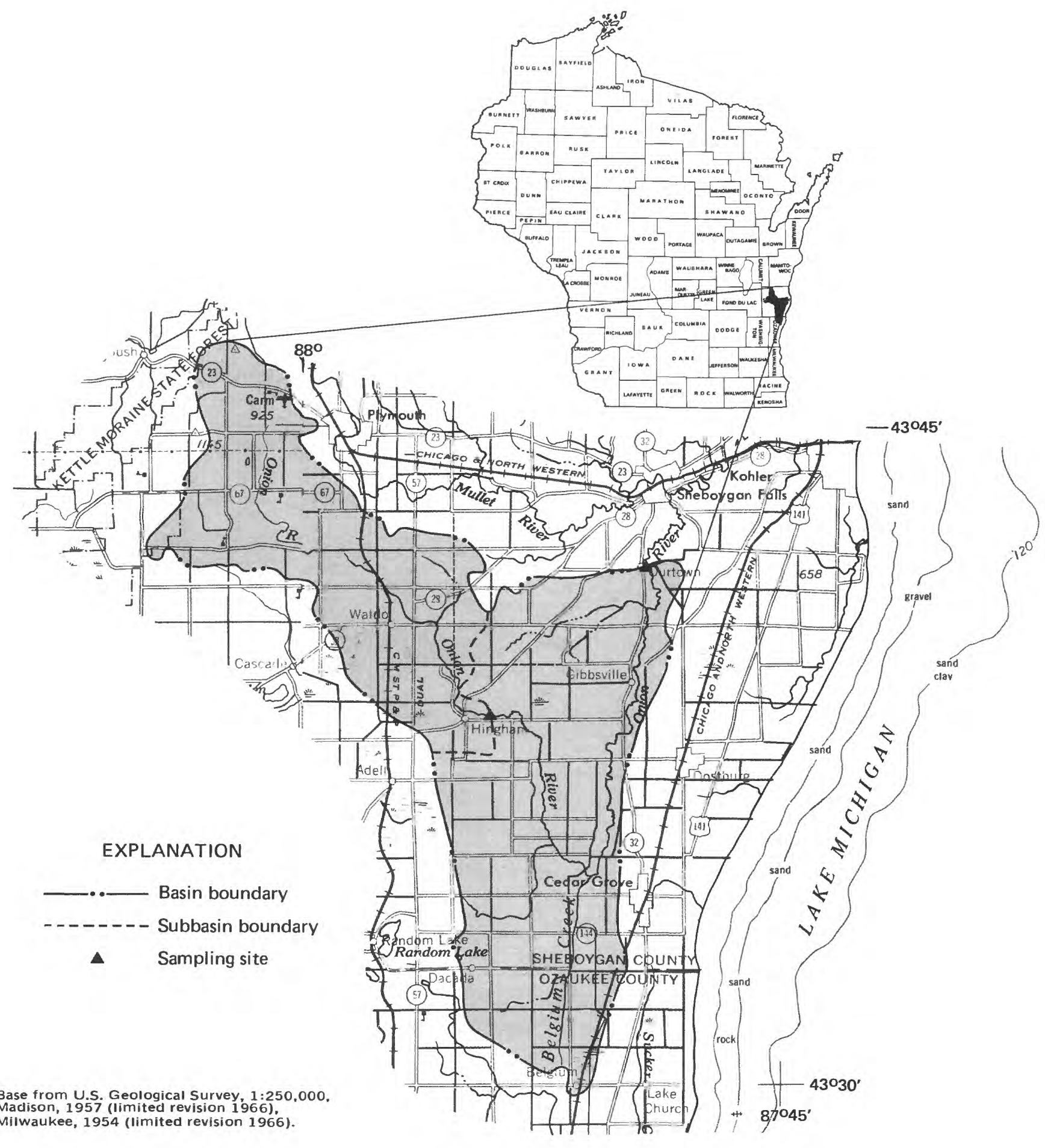

SCALE 1:250,000

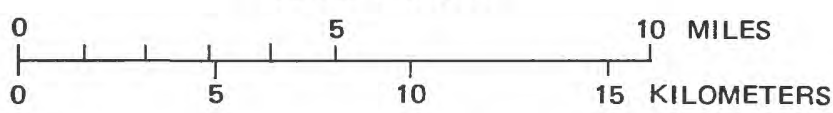

Figure 1. Location of Onion River basin in Wisconsin. 
ly. Point sources of pollution are defined as discharges of waste water from discrete, specific sites such as sewage-treatment-plant and industrial-waste outfalls. Nonpoint sources of pollution are diffuse discharge of pollutants that cannot readily be identified as point sources and include storm water and snowmelt runoff from urban and rural land surfaces, livestock operations, and construction activities. Although substandard septic systems are categorized as point sources, because of their minor contribution to the total nutrient load, nonpoint sources, as used in this report, include these septic systems.

An intensive water-quality-monitoring program in the Onion River basin was started by the U.S. Geological Survey in cooperation with the DNR in December 1978 and was continued through September 1980. Two water-quality and stream-discharge monitoring sites (fig. 1) were selected: one at Hingham, upstream of the problem area, and one near Sheboygan Falls, downstream of the problem area. The drainage areas are 37.2 and $94.1 \mathrm{mi}^{2}$, respectively.

\section{Purpose and Scope}

The purpose of this report is to define the relationship between water quality and nonpointsource discharges in the Onion River. The scope includes determination of (1) streamflow, (2) the annual loadings of suspended sediment, nitrogen, and phosphorus; (3) water temperature and dissolved-solids concentrations; and (4) miscellaneous water-quality characteristics, including $\mathrm{pH}$, biochemical oxygen demand, periphyton biomass, and concentrations of dissolved oxygen, fecal coliform and fecal streptococci bacteria, alkalinity, chloride, trace metals, and pesticides.

\section{Acknowledgments}

The authors would like to thank James S. Baumann, Wisconsin Department of Natural Resources, for his assistance on this project, as well as land owners Rueben Tenhaken and Eugene Kretschmann, who allowed gaging stations to be installed on their property.

\section{PHYSICAL SETTING}

\section{Topography and Drainage}

The Onion River begins southwest of Plymouth on the eastern edge of the Kettle Moraine State Forest at an altitude of about 1,080 ft. It flows southeastward to near Cedar Grove where it turns abruptly northward to Sheboygan Falls and the Sheboygan River, which flows into Lake Michigan. The Onion River basin is an undulating plain sloping toward Lake Michigan. The valleys and uplands are broad and gently sloping and trend parallel to Lake Michigan. Relief in the headwaters area is generally as much as $200 \mathrm{ft}$; downstream of Hingham the relief is generally less than $50 \mathrm{ft}$. Along the western edge of the lower Onion River basin, relief is as great as $150 \mathrm{ft}$.

\section{Stream-Channel Characteristics}

The Onion River from the headwaters to the gaging station at Hingham is $15.4 \mathrm{mi}$ long and has a gradient of $8.8 \mathrm{ft} / \mathrm{mi}$. From Hingham to the gaging station near Sheboygan Falls the river is $19.7 \mathrm{mi}$ long, with a gradient of $5.6 \mathrm{ft} / \mathrm{mi}$. At Hingham the stream is about $50 \mathrm{ft}$ wide and about $1 \mathrm{ft}$ deep; at the Sheboygan Falls gaging station the stream is about $100 \mathrm{ft}$ wide and 1 to $2 \mathrm{ft}$ deep. The river is a series of pools and riffles. The bed material consists of sand, silt, clay, and cobbles with a few boulders. At the Sheboygan Falls station, boulders are common.

Impoundments are located at Hingham and at Waldo. The impoundment at Hingham has a surface area of 38 acres with a maximum depth of $6 \mathrm{ft}$ (Wisconsin Department of Natural Resources, 1972). The impoundment at Waldo has a surface area of 40 acres and a maximum depth of $7 \mathrm{ft}$.

\section{Climate}

The climate is a continental type with four definite seasons (Wisconsin Department of Agriculture, 1961). Winters are cold and snowy, summers have periods that are hot and humid, and spring and fall are at times moderate mixtures of summer and winter. Temperatures and precipitation recorded at Plymouth (fig.1), range from a mean monthly temperature of $19.9^{\circ} \mathrm{F}$ in January, to $71.0^{\circ} \mathrm{F}$ in July. The mean annual temperature is $45.8^{\circ} \mathrm{F}$. The average annual precipitation is 28.3 in. (1930-59) with

${ }^{1}$ Enacted under Section 208 of Public Law 93-500. 
February the driest month (1.41 in.) and June the wettest ( 3.50 in.). About 53 percent of the average precipitation falls from May through September. Snowfall averages $46.6 \mathrm{in}$. annually.

Precipitation values in this report are the arithmetic means of the National Weather Service stations at Sheboygan, Plymouth, and West Bend, except as explained in the text. The average annual precipitation for these stations is $29.8 \mathrm{in}$. for 30 years of record (Environmental Data Service, 1973).

Total precipitation for the 1979 and 1980 water years was $33.1 \mathrm{in}$. and $36.8 \mathrm{in}$., which is $3.30 \mathrm{in}$. more than normal during the 1979 water year and 7.0 in. more than normal during the 1980 water year.

The maximum recorded snow depths at Sheboygan during the study were 28 in. during the 1979 water year and 6 in. during the 1980 water year. The moisture equivalents in the snowpack for the study period were also significantly different. On March 1, 1979, before snowmelt, there was an average moisture equivalent in the snowpack at Green Bay, (60 mi to the north), and Milwaukee (45 mi to the south) of 4.4 in. (U.S. Department of Commerce, 1979). On March 8, 1980, however, the moisture equivalent in the snowpack at these same two stations averaged only 0.3 in. (U.S. Department of Commerce, 1980).

\section{Geology}

Surficial deposits in the Onion River basin are glacial drift consisting of clay, sand, gravel, and boulders deposited by the Lake Michigan glacial lobe during the Pleistocene Epoch. These deposits generally are from 20 to $100 \mathrm{ft}$ thick, except in the Kettle Moraine State Forest area where they can be as much as $250 \mathrm{ft}$ thick.

Bedrock underlying the basin is dolomite of Silurian age and is about 250 to $500 \mathrm{ft}$ thick across the basin. The Silurian Dolomite is underlain by the Maquoketa Shale of Ordovician age.

\section{Soils}

Soils of the Onion River basin are described by Engel, Roberts, and Steingraeber (1978) for Sheboygan County and by Parker, Kurer, and Steingraeber (1970) for Ozaukee County.
Two major soil associations, the Casco-FoxRodman and the Hochheim-Theresa, occur upstream of Waldo. The Casco-Fox-Rodman association is well-drained to excessively drained soils with a subsoil of mainly silty clay loam to sandy clay loam or gravelly sandy loam underlain by stratified gravel and sand outwąsh. The Hochheim-Theresa association is well-drained soils with a subsoil of mainly clay loam or silty clay loam underlain by gravelly sandy loam glacial till. These soils have a slight or moderate erosion potential.

The Kewaunee-Waymor-Manawa association occurs predominantly in the river basin downstream of Waldo. These soils are well drained to somewhat poorly drained soils with a subsoil of mainly clay loam to clay underlain by loam or silty clay loam glacial till. This soil association has a moderate to severe erosion potential.

\section{Land Use}

Agriculture is the principle land use in the Onion River basin (Wisconsin Department of Natural Resources, 1981); dairy farming and cash cropping are the two major uses (table 1). The basin supports about 11,600 animal units ${ }^{2} ; 2,800$ animal units are in the basin upstream of Hingham at a density of 75 per square mile and about 8,790 animal units are in the basin downstream of Hingham at a density of 154 per square mile.

Land use in the basin as determined by J. S. Bauman (written commun., Wisconsin Department of Natural Resources, 1981) for the Onion River basin is shown in table 1. The potential exists for greater nonpoint sources of runoff downstream of Hingham because of more cropland and high animal density compared to more woodland and low animal density upstream of Hingham.

\section{METHODS OF STUDY}

Gaging stations to measure streamflow, temperature, and specific conductance were installed in December 1978 at Hingham and at Sheboygan Falls. Isco Model $1680^{3}$ automatic water samplers were installed to collect samples during storm runoff for analyses of suspended sediment and nutrient concentrations. A local observer collected weekly suspended-sediment samples during nonstorm periods.

${ }^{2}$ One animal unit equals $1,000 \mathrm{lb}$ live weight.

3 The use of trade names in this report is for identification purposes only and does not constitute endorsement by the U.S. Geological Survey. 
Streamflow characteristics for Onion River at Hingham (04085813) and Sheboygan Falls (04085845) during the study were supplemented with long-term data from the Sheboygan River at Sheboygan gaging station $(04086000)$. The station is $12.7 \mathrm{mi}$ downstream of the gaging station on the Onion River near Sheboygan Falls, and has a drainage area of $418 \mathrm{mi}^{2}$.

Precipitation records were obtained from the National Weather Service (U.S. Department of Commerce, 1979, 1980) for stations located at Plymouth, at Sheboygan Falls (fig. 1), and at West Bend (about $25 \mathrm{mi}$ southwest of the basin). Recording rain gages were also located at the gaging stations and were operated only during ice-free periods.

For the collection of nutrient samples, alternate, empty sample bottles in the automatic sampler were pretreated with $1 \mathrm{~mL}$ (milliliter) of mercuric chloride to inhibit biological activity and were remóved from the automatic sampler as soon as possible and chilled to $4^{\circ} \mathrm{C}$. Four samples pretreated with mercuric chloride and four untreated samples were selected to define the temporal concentration curves for suspended sediment and nutrients throughout the stream discharge hydrograph. Those treated with mercuric chloride were analyzed for:

- Nitrite nitrogen

- Nitrite plus nitrate nitrogen

- Ammonia plus organic nitrogen

Ammonia nitrogen

- Phosphorus

- Phosphorus, orthophosphate

The four untreated samples were filtered and analyzed for:

- Chloride

- Alkalinity

- Dissolved solids, residue on evaporation at $180^{\circ}$

Samples were selected from the remaining bottles for suspended-sediment analyses. All water-quality data were published in the Water Resources Data for Wisconsin, Water Years 1979 and 1980 (U.S. Geological Survey, 1980 and 1981).

Several stream cross-section samples were manually collected concurrently with automatically collected samples that cover a range of stream discharges. This method insured that the automatically collected samples represented the average water quality in the stream cross section. These samples were collected using the equal-width-increment method described by Guy and Norman (1970).

Samples were collected manually at 6-week intervals and the following water-quality characteristics were determined:

- $\mathrm{pH}$

- Concentration of fecal coliform bacteria

- Dissolved-oxygen concentration

- Concentration of fecal streptococci bacteria

- Biochemical oxygen demand--5 day

- Turbidity (1980 water year only)

- Nitrite nitrogen

- Nitrite plus nitrate nitrogen

- Ammonia plus organic nitrogen

- Ammonia nitrogen

- Phosphorus

- Phosphorus, orthophosphate

Dissolved-oxygen concentrations and $\mathrm{pH}$ were determined in the field at the time of sampling with a Leeds and Northrup Model $7417 \mathrm{pH}$ meter and Yellow Springs Model 54 dissolved-oxygen meter. Samples for biochemical oxygen demand, fecal coliform, and fecal streptococci bacteria were chilled to $4^{\circ} \mathrm{C}$ and analyzed later at the U.S. Geological Survey laboratory at Madison. Samples for turbidity and suspended-sediment concentration were also analyzed at the Madison laboratory. Samples for the nitrogen and phosphorus species were treated with $1 \mathrm{~mL}$ of mercuric chloride and the chlorophyll $a$ and $b$ samples were chilled to $4{ }^{\circ} \mathrm{C}$ and sent to the National Water Quality Laboratory at 
Doraville, Ga., for analyses.

\section{STREAMFLOW}

Streamflow characteristics for the Onion River stations and Sheboygan River at Sheboygan are summarized in table 2. Daily streamflow data for the Onion River stations for the 1979 and 1980 water years are shown in tables 10 and 11 .

Based on the discharges at the Sheboygan River gaging station, discharge for the 1979 water year was about 60 percent above average; the 1980 discharges were about 5 percent below average.

Stream discharge was greater during the 1979 water year than during the 1980 water year. Total precipitation during the 1980 water year was greater than that of 1979 , but the 1979 water year had higher winter precipitation. Greater winter precipitation combined with lower evapotranspiration in winter resulted in more runoff and, therefore, greater 1979 water-year stream discharge. This is shown in table 3.

The runoff upstream of Hingham was generally greater than that of the area downstream during most months. However, during the 1979 water year, runoff in the Onion River basin upstream of Hingham was 2.60 in. less than the runoff downstream of Hingham; in the 1980 water year, runoff was 3.50 in. greater. The differences in runoff probably can be attributed to differences in precipitation patterns in the basins and to the differences in ground-water discharge. To illustrate the effects of these two variables, a double-massaccumulation plot of runoff for the two areas is shown in figure 2. The slope of the line indicates a higher runoff rate upstream of Hingham than downstream except during the periods February 28 to April 1, 1979, and during September 1980.

Hydrograph separations, such as those described by Linsley, Kohler, and Paulhus, (1975), made for both stations are shown in figures 3 and 4 . Base flow and storm runoff components are summarized in table 4. The separations show that base flow at Onion River at Hingham is a greater percentage of total stream discharge than near Sheboygan Falls. Base flow is discharge that is composed of ground-water runoff.

Streamflow extremes occurred during the 1979 water year; the minimum 7-day mean discharge at both stations occurred in September, 1979. Based on the recorded discharge for the Sheboygan River at Sheboygan, the minimum flows were at the 76 percent flow duration point--about double the average 7-day low flow that occurs on the average of once every 2 years $\left(Q_{7,2}\right)$. The maximum discharge at the Onion River stations occurred in March 1979, and was caused by melting of the snowpack with a high moisture content. The peak discharge of 6,460 $\mathrm{ft}^{3} / \mathrm{s}$ at the Sheboygan River gaging station (March $31,1979)$ was between expected peak discharges for recurrence intervals of 5 and 10 years $\left(5,310 \mathrm{ft}^{3} / \mathrm{s}\right.$ and $6,940 \mathrm{ft}^{3} / \mathrm{s}$ ), respectively (Conger, 1981). During 1980 the peak discharges in September at the Onion River stations were about one-half the recurrence interval of a mean annual flood.

Holmstrom (1979) found that, during extended drought periods, (which did not occur during the study), there is little ground-water discharge downstream of Hingham (table 5). Table 5 shows that the Onion River loses water between Gibbsville and Sheboygan Falls.

\section{WATER QUALITY}

The water in the Onion River at Hingham and at Sheboygan Falls is of the calcium-magnesium bicarbonate type; alkalinity values are typical of those in streams tributary to Lake Michigan in east-central Wisconsin (alkalinity as calcium carbonate ranges from 80 to $360 \mathrm{mg} / \mathrm{L}$ (milligrams per liter).

Water quality from numerous springs in the headwaters of the Onion River is capable of maintaining a trout fishery (Wisconsin Department of Natural Resources, 1979). From the headwaters to County Highway N, $0.1 \mathrm{mi}$ upstream from the impoundment at Waldo, the stream is classified as a Class II trout stream--one in which there is some natural reproduction but moderate to heavy fish stocking is required to maintain a good fishery. Stream temperatures are raised at the impoundments at Waldo and Hingham above the maximum temperature allowable to maintain a trout fishery. Downstream of Waldo, the Onion River supports a warm-water fishery.

The poorest water quality (for most uses) in the basin resulting from strong organic pollution and high nutrient levels (Wisconsin Department of Natural Resources, 1979) is in Belgium Creek and in the main stem of the Onion River downstream of 


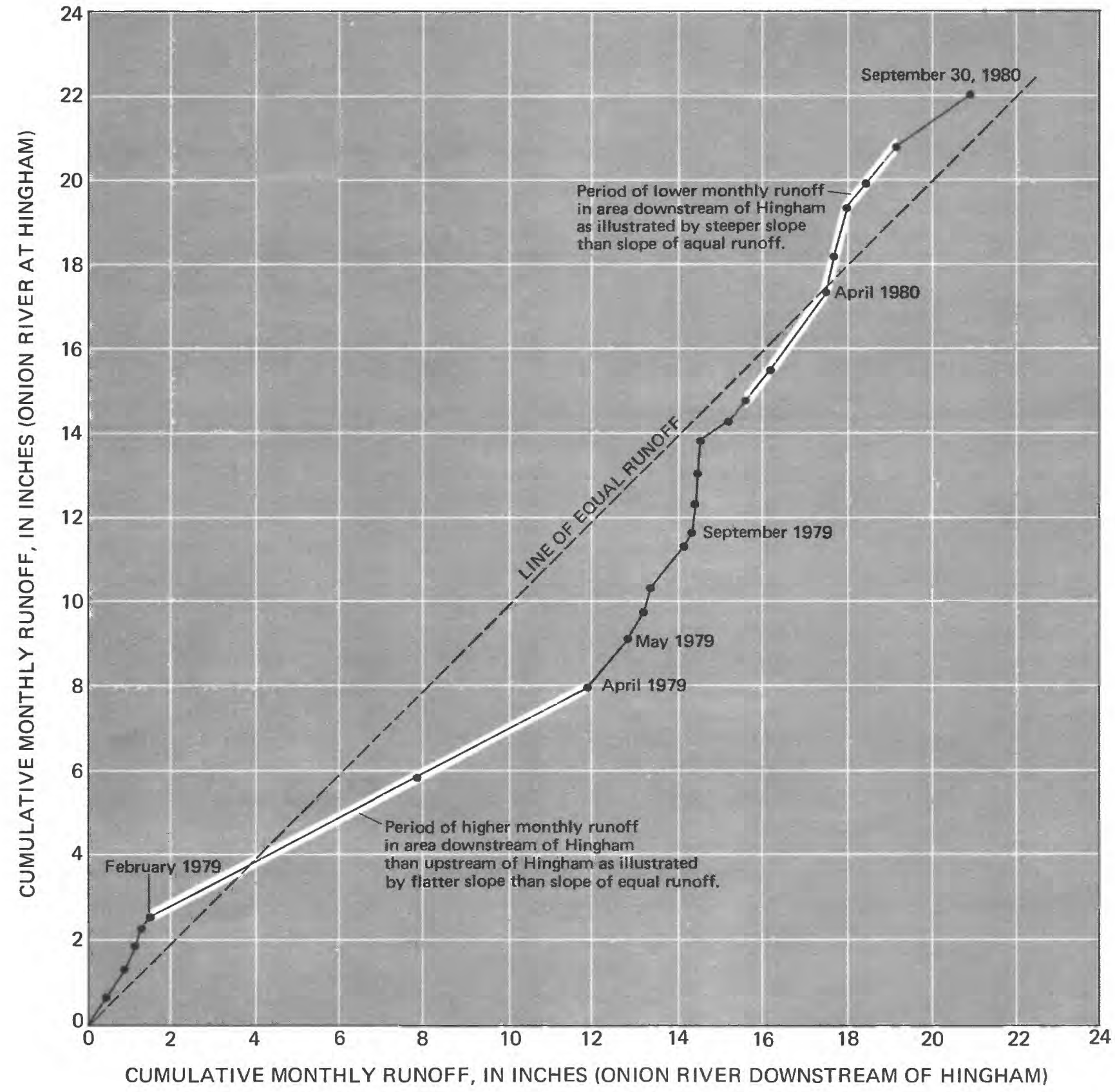

Figure 2. Double mass-accumulation curves for monthly runoff at Onion River at Hingham and Onion River downstream of Hingham. 

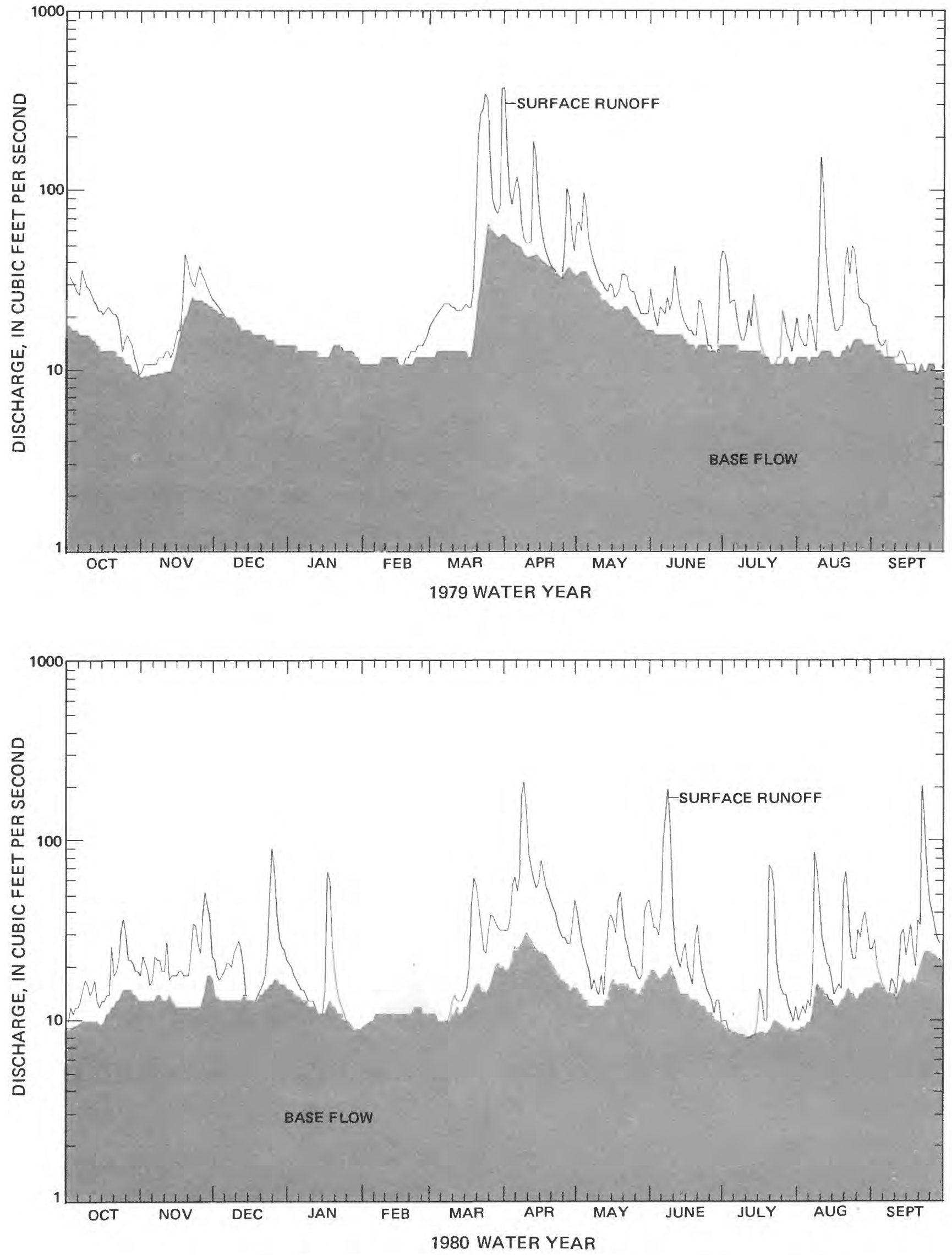

Figure 3. Hydrographs of base flow and surface runoff for Onion River at Hingham, 1979 and 1980 water years. 

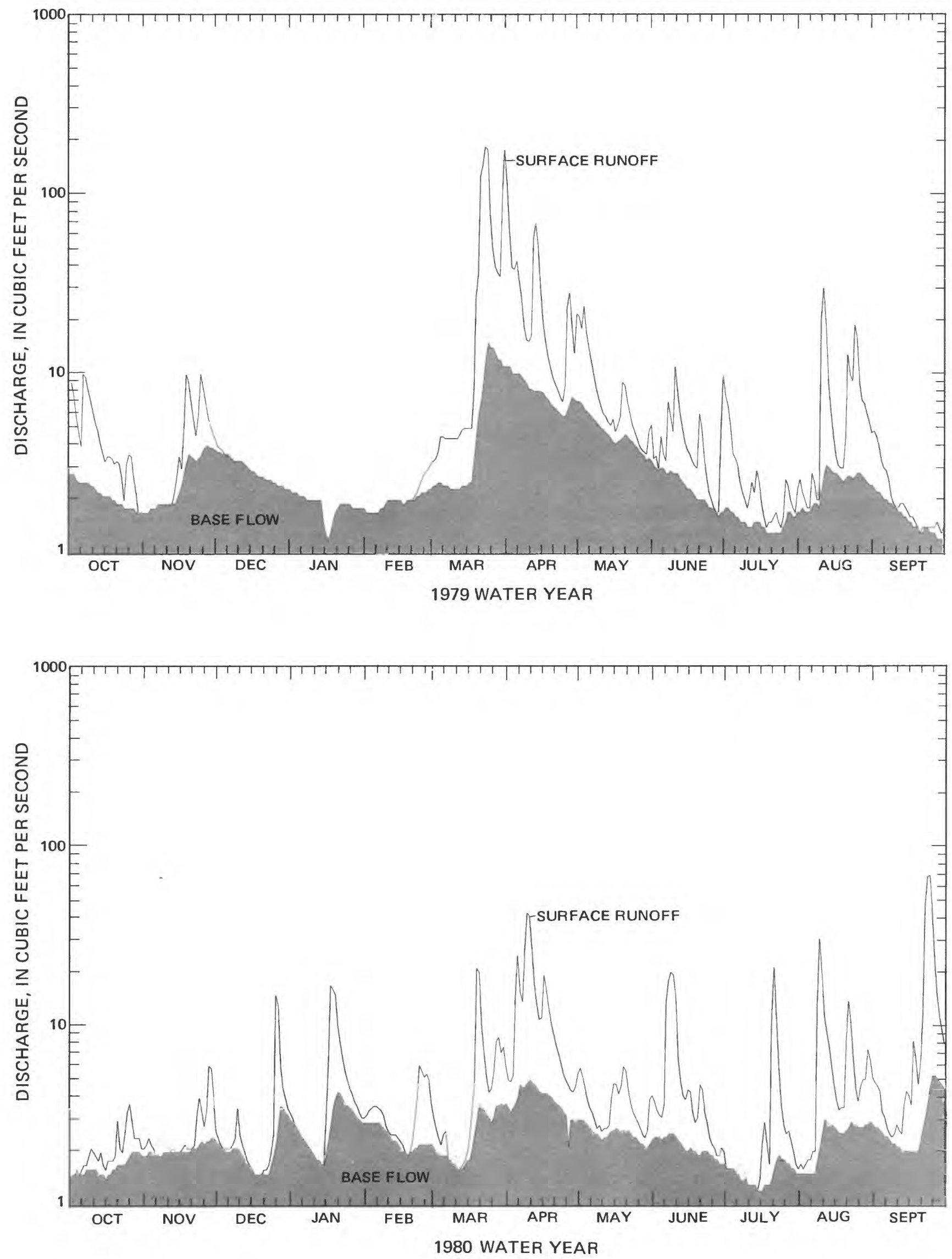

Figure 4. Hydrographs of base flow and surface runoff for Onion River near Sheboygan Falls, 1979 and 1980 water years. 
the confluence with this creek.

Point sources of pollution include waste-water treatment plants at the villages of Waldo, Hingham, Belgium, and Gibbsville. A vegetable canning company near Belgium discharges into a sewage lagoon that in turn discharges into Belgium Creek. The preserving company discharged wastes intermittently from April 25, to October 30, 1979, and from about June 1 to October 30, 1980 (D. Sauer, Wisconsin Department of Natural Resources, oral commun., January 5, 1982).

In a preliminary assessment of the basin in 1978 the Southeast District of the DNR made dry and wet weather surveys of the Onion River on September 12 and October 30 to determine water-quality trends. They found that phosphorus concentrations during runoff progressively increased in a downstream direction. The results of their data are shown in figure 5.

Complete water-quality data for the Onion River at Hingham and Sheboygan Falls for the 1979 and 1980 water years are given in tables 12 and 13 . throughout the study. Turbidity changed drastically from very low during winter to exceptionally high during summer when the carp are most active. During 1980 at Sheboygan Falls, turbidity ranged from 5 (March 1) to 80 (June 27) Jackson turbidity units. The Wisconsin Department of Natural Resources (1979), in their preliminary assessment of the basin, also recognized increased turbidity due to carp activity.

Carp are a bottom-dwelling fish species whose activities can cause problems in the aquatic environment. They uproot aquatic vegetation and roil the water while foraging for food (Burns, 1966). The bulk of their vegetation diet consists of filamentous green algae and higher aquatic plants (Dill, 1944; Wales, 1943). Generally inactive at water temperatures below $3.5^{\circ} \mathrm{C}$ (Sigler, 1958), as the water begins to warm in the spring they move into the shallows to begin spawning. Spawning begins at water temperatures of about $15.5^{\circ} \mathrm{C}$, and becomes most active between $18.5^{\circ}$ and $20^{\circ} \mathrm{C}$ (Greeley, 1927; Sigler 1958). When these temperatures are attained spawning occurs both day and night and is accompanied by much commotion and splashing (Burns, 1966).

Numerous carp were observed at both stations

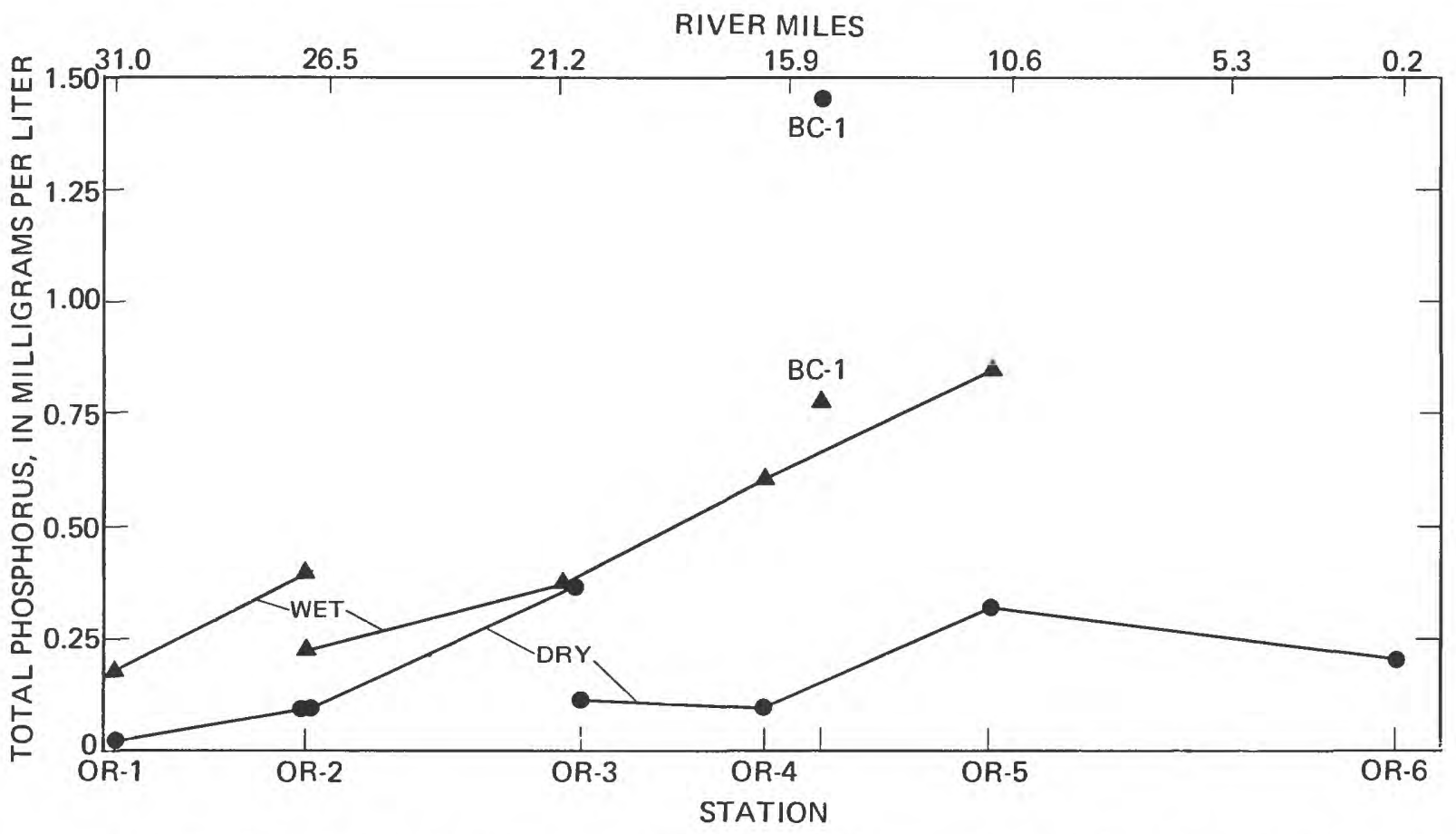

Note: The Onion River at Sheboygan Falls gaging station is at 3.9 river miles and the Onion River at Hingham gaging station is at 23.6 river miles. The letters and numbers on the abscissa correspond to the DNR sampling stations. "BC-1" refers to a sampling station about 1 mile upstream from the mouth of Belgium Creek. IIlustration courtesy of Jeffrey Bode, Southeast District, Wisconsin Department of Natural Resources.

Figure 5. Total phosphorus concentration trends of dry-and wet-weather surveys for the Onion River. 


\section{Base-Flow Quality}

The chemical quality of the ground-water component of streamflow can be determined from data collected during base-flow periods. The average concentrations of the nitrogen species during baseflow periods were nearly the same in water from the Onion River at Hingham as at Sheboygan Falls. However, the concentrations of orthophosphate and total phosphorus at Sheboygan Falls were about twice those at Hingham (table 6).

Nitrite nitrogen was almost nonexistent in the base-flow water samples. This was not unexpected due to the relatively rapid rate of oxidation of nitrite $\left(\mathrm{NO}_{2}\right)$ to nitrate $\left(\mathrm{NO}_{3}\right)$ under well-aerated conditions during base-flow periods. At Hingham nitrate nitrogen accounted for an average of 38 percent of the total nitrogen present. Organic nitrogen accounted for 52 percent and ammonia nitrogen, 10 percent. At Sheboygan Falls organic nitrogen accounted for 62 percent of the total nitrogen present, whereas nitrate nitrogen accounted for 31 percent and ammonia nitrogen 7 percent.

Total phosphorus concentrations in water collected from the Onion River at Hingham during base-flow periods ranged from 0.05 to $0.31 \mathrm{mg} / \mathrm{L}$. The median value was $0.17 \mathrm{mg} / \mathrm{L}$. Thirty-five percent of the total phosphorus was orthophosphate phosphorus. At Sheboygan Falls the range was from 0.13 to $0.44 \mathrm{mg} / \mathrm{L}$ with a median of 0.40 $\mathrm{mg} / \mathrm{L}$. Thirty-seven percent of the total phosphorus was orthophosphate phosphorus.

The concentration of total ammonia nitrogen at Hingham ranged from 0 to $0.31 \mathrm{mg} / \mathrm{L}$ with a median of $0.14 \mathrm{mg} / \mathrm{L}$. At Sheboygan Falls concentrations ranged from 0.02 to $0.36 \mathrm{mg} / \mathrm{L}$ with a median of $0.11 \mathrm{mg} / \mathrm{L}$.

\section{Chemical and Physical Characteristics}

\section{Dissolved Solids}

A continuous record of specific conductance was obtained at both stations from April 1979 to September 30,1980 . The daily maximum, minimum, and mean values, and yearly figures are shown in tables 28 and 29. Specific conductance generally is inversely related to stream discharge and directly related to dissolved-solids concentration. At the Onion River at Hingham, the relation between specific conductance and dissolved solids was

$0.557 \times$ specific conductance $(\mu \mathrm{mho} / \mathrm{cm})=$ dissolved solids $(\mathrm{mg} / \mathrm{L})$.

At Onion River near Sheboygan Falls, the relation was

$0.651 \times$ specific conductance $(\mu \mathrm{mho} / \mathrm{cm})=$ dissolved solids $(\mathrm{mg} / \mathrm{L})$.

Dissolved solids loads for the study are shown below.

\section{Water Temperatures}

A continuous record of water temperatures was obtained at both stations during the study. The daily maximum, minimum, and mean values are shown in tables 14 and 15.

\section{Biochemical Oxygen Demand}

Values for 5-day biochemical oxygen demand (BOD) indicate moderate levels of oxidizable organic material in the stream a both Hingham and Sheboygan Falls. The 5-day BOD values at Hing-

\section{Total dissolved solids}

Upstream of Hingham

\begin{tabular}{lcccc} 
Water year & Tons & $\begin{array}{c}\text { Tons per square } \\
\text { mile per day }\end{array}$ & Tons & $\begin{array}{c}\text { Tons per square } \\
\text { mile per day }\end{array}$ \\
\hline $\begin{array}{l}1979 \text { (May through } \\
\text { September) }\end{array}$ & 3,170 & 0.56 & 4,500 & 0.52 \\
1980 & 8,610 & .63 & 14,000 & .67 \\
\hline
\end{tabular}


ham ranged from 1.1 to $6.3 \mathrm{mg} / \mathrm{L}$; at Sheboygan Falls the 5-day BOD values ranged from 2.2 to 8.7 $\mathrm{mg} / \mathrm{L}$.

\section{Autotrophic Index}

Ratios of periphyton biomass to chlorophyll, known as the autotrophic index, indicated low levels of available organic loading at Hingham and high levels at Sheboygan Falls. At Hingham the ratios ranged from 47 to 155; the range at Sheboygan Falls was from 149 to 70,500 . The autotrophic index is computed by dividing ash-free dry weight $\left(\mathrm{g} / \mathrm{m}^{2}\right)$ of the periphyton sample by its chlorophyll $a$ content $\left(\mathrm{g} / \mathrm{m}^{2}\right)$. Values greater than 100 indicate some organic contamination; values range into the thousands for organically contaminated streams.

\section{Indicator Bacteria}

Wisconsin water-quality standards for recreational waters for fecal coliform bacteria are the same as those of the U.S. Environmental Protection Agency, 1977: "The geometric mean of not less than 5 samples within a 30 -day period shall not exceed 200 per $100 \mathrm{~mL}$ nor shall more than $10 \%$ of total samples during any 30-day period exceed 400 per 100 mL."

Concentrations of fecal coliform and fecal streptococci bacteria indicate fecal contamination and the possible presence of pathogenic organisms. At Hingham the values for concentrations of fecal coliform bacteria ranged from 4 to 830 colonies per $100 \mathrm{~mL}$ of sample with a median of 113 ; those for fecal streptococci bacteria ranged from 11 to 3,300 colonies per $100 \mathrm{~mL}$ with a median of 57 .

Concentrations of fecal coliform bacteria at Sheboygan Falls ranged from 25 to 13,000 colonies per $100 \mathrm{~mL}$, with a median of 170 ; those for fecal streptococci bacteria ranged from 12 to 2,600 colonies per $100 \mathrm{~mL}$ with a median of 200 .

\section{Other}

The concentrations of pesticides, polychlorinated biphenyls, and polychlorinated naphthalenes at Hingham in an unfiltered water sample collected June 28, 1979, and a sample of bed material collected August 15, 1980, were less than the detection limit.
Concentrations of pesticides in unfiltered water and bed-material samples at Sheboygan Falls were less than the detection limit. The concentration of polychlorinated biphenyls (PCB) was $1 \mu \mathrm{g} / \mathrm{kg}$ in a bed-material sample collected on August 15, 1980.

\section{RELATION OF SUSPENDED SEDIMENT AND NUTRIENTS TO STREAMFLOW}

Suspended-sediment and nutrient loads, computed by streamflow and concentration-integration techniques described by Porterfield (1972) are given in tables 16-29. The loads for constituents in the individual runoff events are shown in table 7. At Hingham, runoff from five storms during the 1979 water year was sampled for selected chemical constituents; at Sheboygan Falls runoff from six storms was sampled. In the 1980 water year runoff from seven storms was sampled at each station.

Annual yields of suspended sediment and nutrients (various forms of nitrogen and phosphorus) were greater in the 1979 water year than in the 1980 water year. To assess the impact of the drainage area on suspended sediment and nutrient yields downstream of Hingham, the discharges and loads at Hingham were subtracted from those at Sheboygan Falls; the results are shown in table 8 . The 2-year average suspended-sediment yield for Hingham was 72 tons $/ \mathrm{mi}^{2}$; for the basin downstream from Hingham it was 89 tons $/ \mathrm{mi}^{2}$. The effect of the impoundments at Hingham and Waldo on suspended-sediment and nutrient loads is unknown. Because the stream discharge in 1979 was 60 percent above average and near normal in 1980, and precipitation for those years was $3.3 \mathrm{in}$. and $7.0 \mathrm{in}$. more than normal, respectively, the sediment and nutrient loads may be greater than the long-term average for the Onion River. The suspended-sediment yields for the Onion River stations are greater than the long-term average of $62 \mathrm{ton} / \mathrm{mi}^{2}$ for Sheboygan River at Sheboygan reported by Hindall (1975).

An initial comparison of suspended-sediment and nutrient yields at Hingham and Sheboygan Falls may be misleading. During the 1979 water year runoff at Sheboygan Falls was 13.33 in. and at Hingham, 11.75 in. The greater runoff at Sheboygan Falls would result in higher loads if the concentrations for the same constituents at both stations were equal. During the 1980 water year, the runoff at Sheboygan Falls was 6.58 in.; runoff at Hingham was 10.10 in. If all constituent concentrations at both stations were equal, a lower load would result 
at Sheboygan Falls. The use of double-massaccumulation curves helps eliminate bias due to runoff by providing a comparison of loadings and seasonal changes in loadings between the stations. The double mass-accumulation curves (figs. 6-10) are constructed by plotting the cumulative monthly constituent yield against cumulative inches of runoff.

The slopes of the double mass-accumulation curves for suspended sediment (fig. 6) show a slight difference between the two stations; during the 1979 water year the load at Hingham was slightly more than that downstream. During the 1980 water year the load at Hingham was less.

Loading rates downstream of Hingham were higher than those upstream of Hingham for all nutrients. A significant proportion of this may be attributed to point-source pollution from Belgium Creek. The Wisconsin Department of Natural Resources (1981) estimated that during the 1979 water year the phosphorus contribution to the Onion River from point sources on Belgium Creek (including a vegetable canning company that usually discharges on a seasonal basis from May to September) amounted to $16,000 \mathrm{lb}$; other point sources contributed $700 \mathrm{lb}$. Based on a phosphorus load of $37,300 \mathrm{lb}$ downstream of Hingham, the contribution of phosphorus from these point sources represents 45 percent of the total load. Upstream of Hingham, point-source contributions of phosphorus were estimated to be $2,200 \mathrm{lb}$ or 18 percent of the total load. Nonpoint contribution of phosphorus amounted to $362 \mathrm{lb} / \mathrm{mi}^{2}$ downstream of Hingham, compared to $272 \mathrm{lb} / \mathrm{mi}^{2}$ upstream of Hingham. The double mass-accumulation curves for the area downstream of the Hingham station (figs. 9 and 10) show greater fluctuations than the curves (figs. 6-8) for the area upstream of Hingham. This may be due to point-source contributions from Belgium Creek.

In general, the curves (figs. 8 and 10) for nitrite plus nitrate nitrogen and ammonia nitrogen tend to flatten out during the growing season, possibly reflecting the utilization of the nutrients by aquatic plants. The shapes of the curves for phosphorus and orthophosphate (figs. 7 and 9) and organic nitrogen (figs. 8 and 10) closely follow the shapes of the suspended-sediment curves illustrating the association of the nutrients with suspended sediment.

The loading rates of dissolved solids, in tons per square mile, from Onion River at Hingham and the
Onion River downstream of Hingham appear similar. However, the area above Hingham had more runoff than the area downstream, producing a disproportionate load at Hingham. A plot of double mass-accumulation curves (fig. 11), however, again illustrates a higher loading rate of dissolved solids downstream of Hingham.

\section{Water-Quality Criteria}

Concentration standards for nutrients, trace metals, and pesticides have been established by the U.S. Environmental Protection Agency (1977) and the Wisconsin Department of Natural Resources for the protection of freshwater aquatic life. Ammonia nitrogen concentrations at both Hingham and Sheboygan Falls did not exceed the DNR criterion of $0.04 \mathrm{mg} / \mathrm{L}$ of un-ionized ammonia $\left(\mathrm{NH}_{3}-\mathrm{N}\right)$ (Schuettpelz and Harpt, Wisconsin Department of Natural Resources, written commun., 1980); only the March 1980 samples reached the U.S. Environmental Protection Agency's criterion of $0.02 \mathrm{mg} / \mathrm{L}$. Concentrations of un-ionized ammonia can be determined from analyses of ammonia nitrogen $\left(\mathrm{NH}_{3}\right.$ $+\mathrm{NH}_{4}{ }^{+}$) if the $\mathrm{pH}$ and water temperature of the samples are known. However, the resulting concentrations may be low due to possible loss of gaseous ammonia from the sample before analysis.

The U.S. Environmental Protection Agency (1977) has suggested that to prevent biological nuisance growths the following concentrations of total phosphorus should not be exceeded:

$0.1 \mathrm{mg} / \mathrm{L}$ for streams not discharging into lakes or impoundments,

$0.05 \mathrm{mg} / \mathrm{L}$ in any stream at the point where it enters a lake or reservoir,

\section{$0.025 \mathrm{mg} / \mathrm{L}$ within a lake or reservoir.}

At Hingham during base flow, four of five samples analyzed exceeded the EPA criterion; at Sheboygan Falls all base-flow samples exceeded the criterion. During storm runoff most samples at both stations exceeded the criterion.

At both stations, concentrations of trace metals (tables 12 and 13) from the water-column samples, June 28, 1979, and from bed-material samples August 15, 1980, were below the EPA criterion where established. 


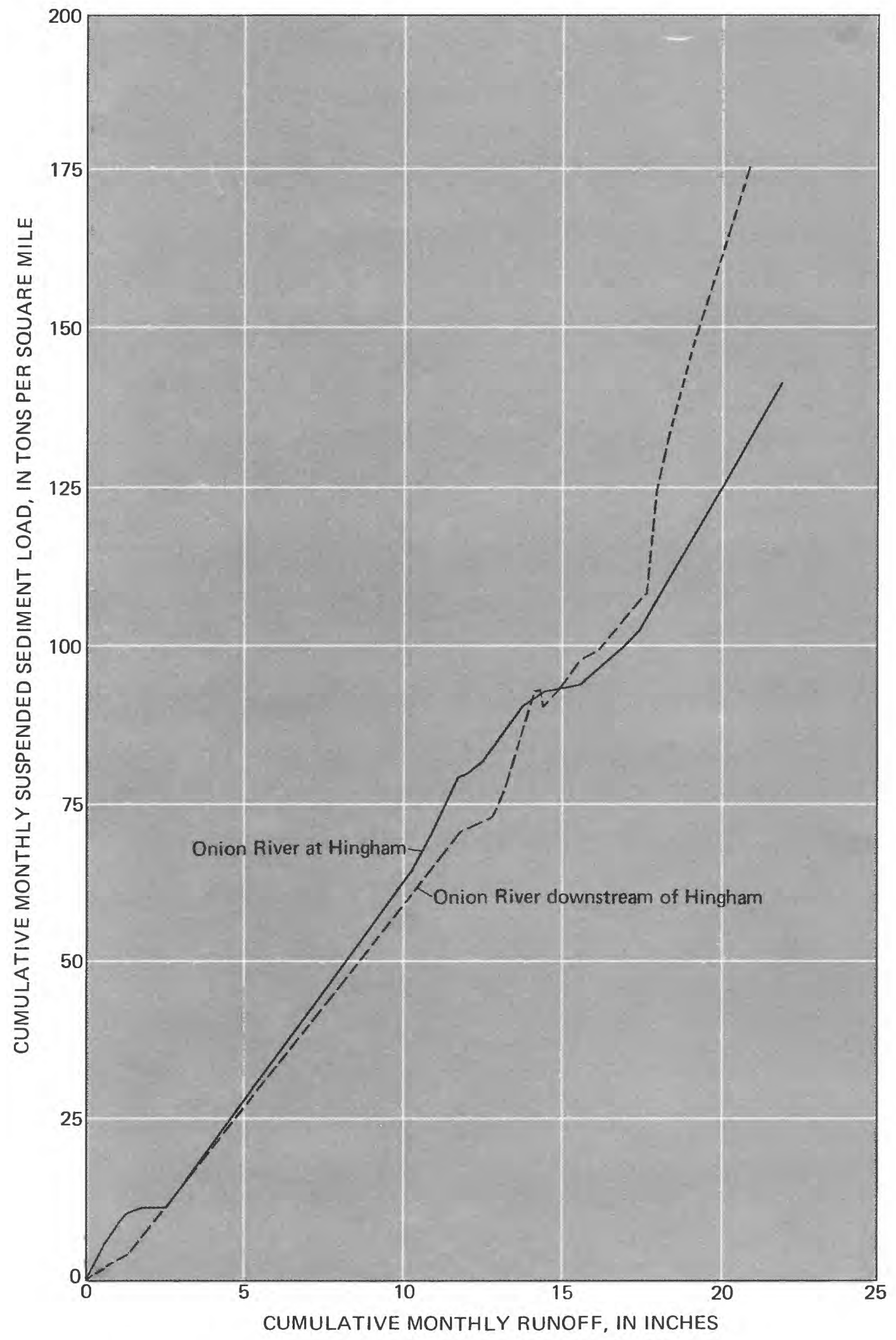

Figure 6. Double mass-accumulation curves of suspended sediment as a function of monthly runoff for Onion River at Hingham and Onion River downstream of Hingham. 


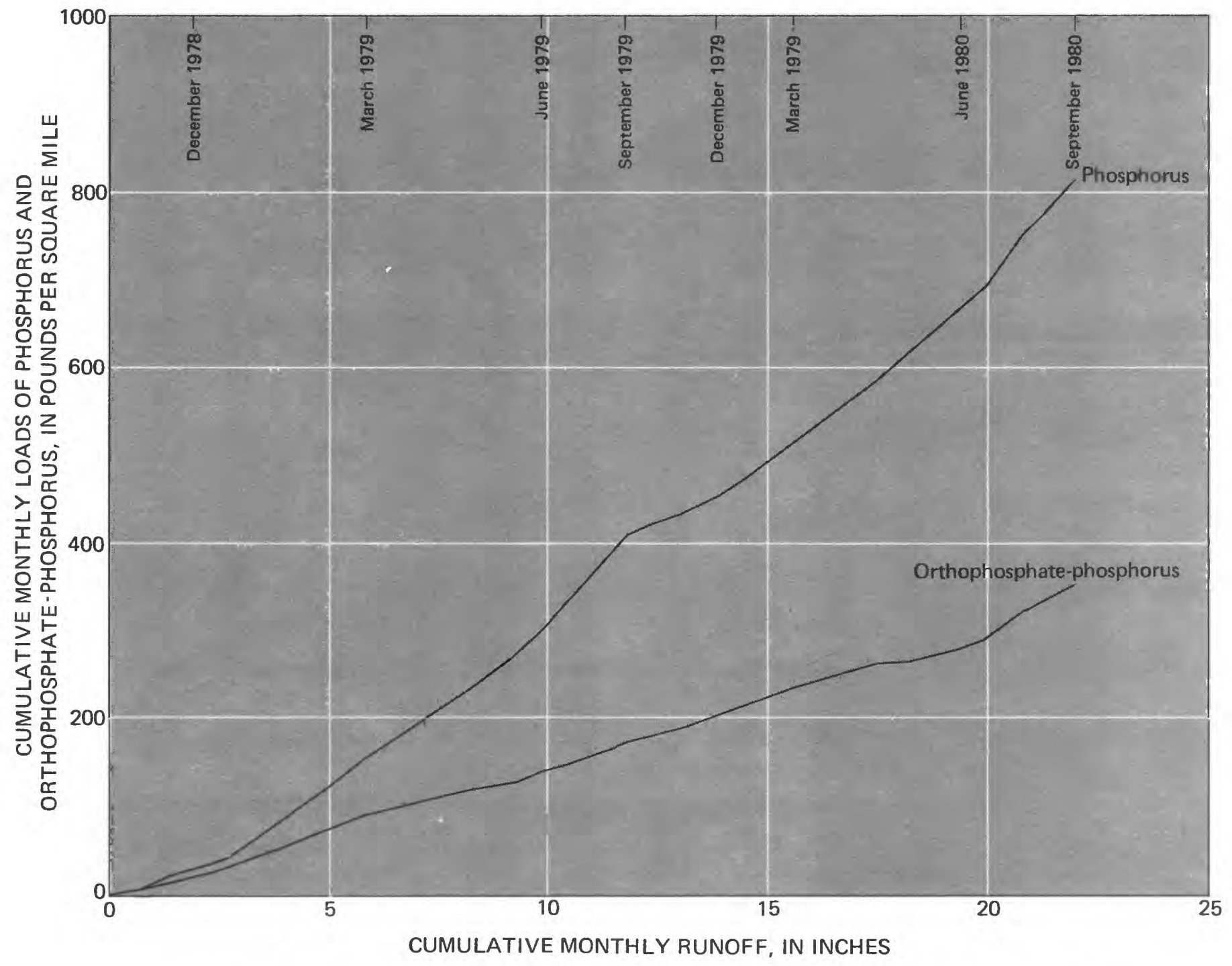

Figure 7. Double mass-accumulation curves of phosphorus and orthophosphate-phosphorus as a function of monthly runoff for Onion River at Hingham. 


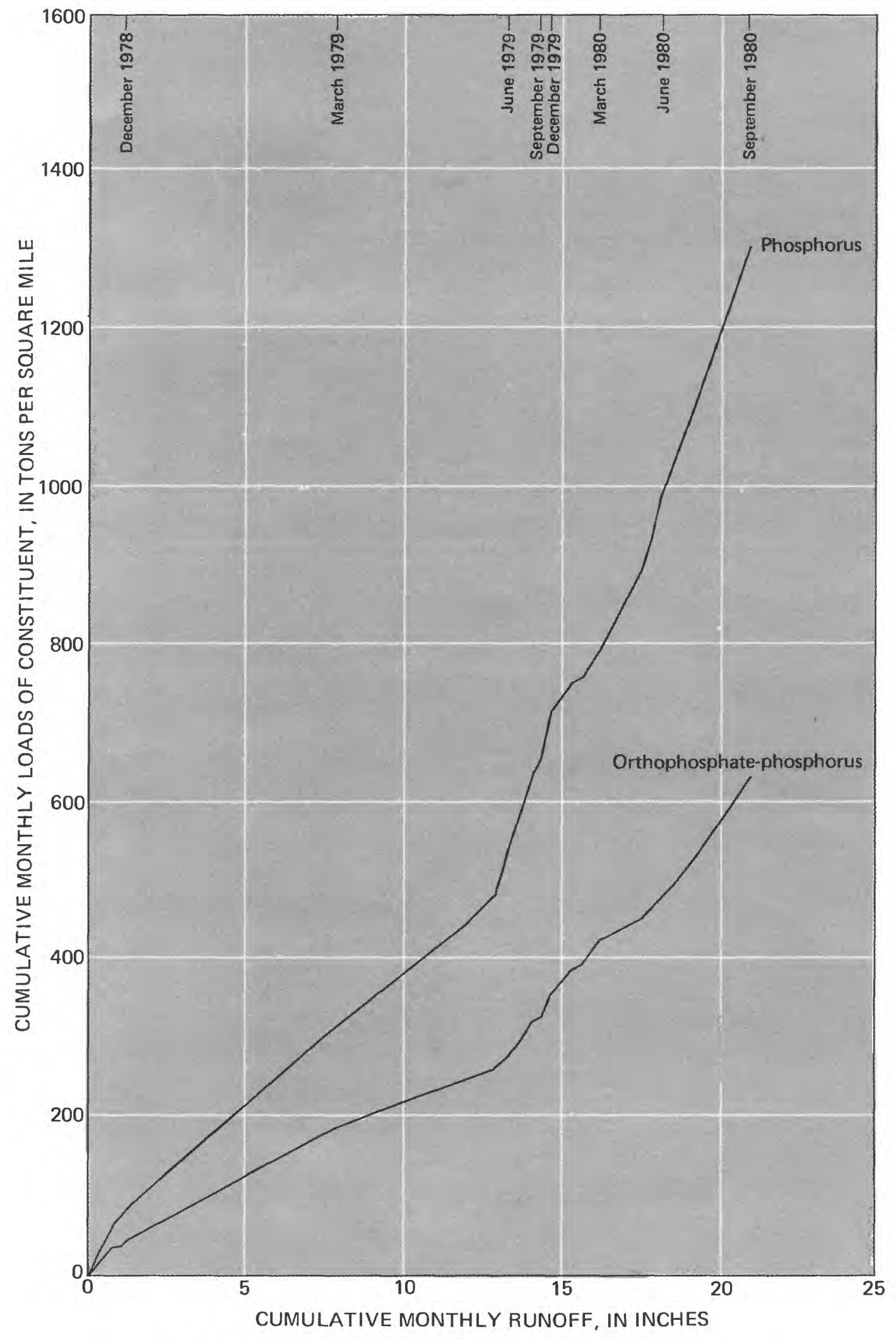

Figure 8. Double mass-accumulation curves of phosphorus and orthophosphate-phosphorus as a function of monthly runoff for Onion River downstream of Hingham. 


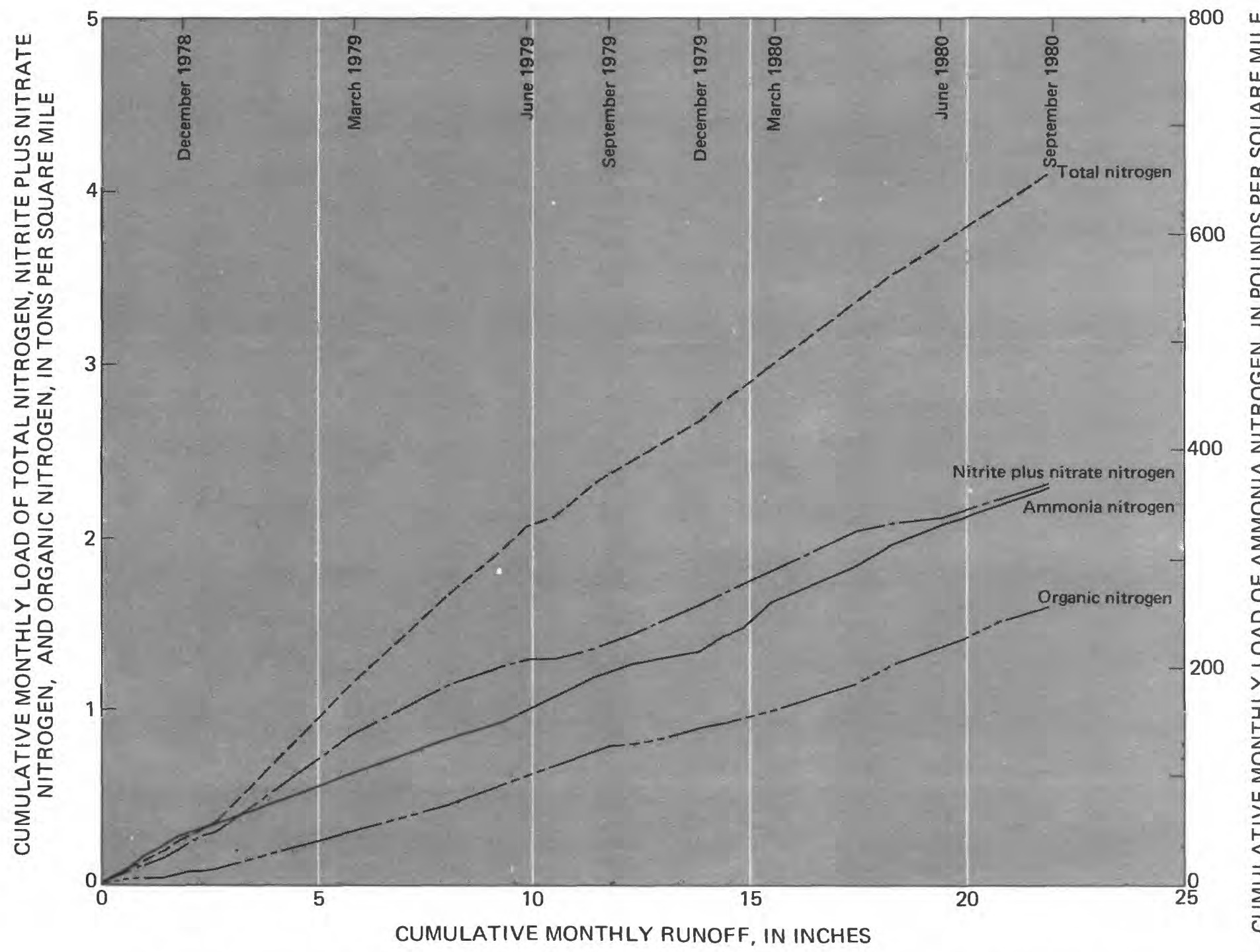

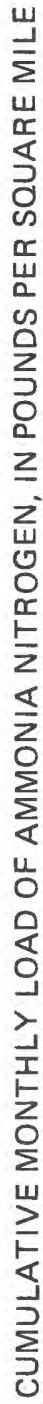

Figure 9. Double mass-accumulation curves of nitrogen, ammonia nitrogen, nitrite plus nitrate nitrogen and organic nitrogen as a function of monthly runoff for Onion River at Hingham. 


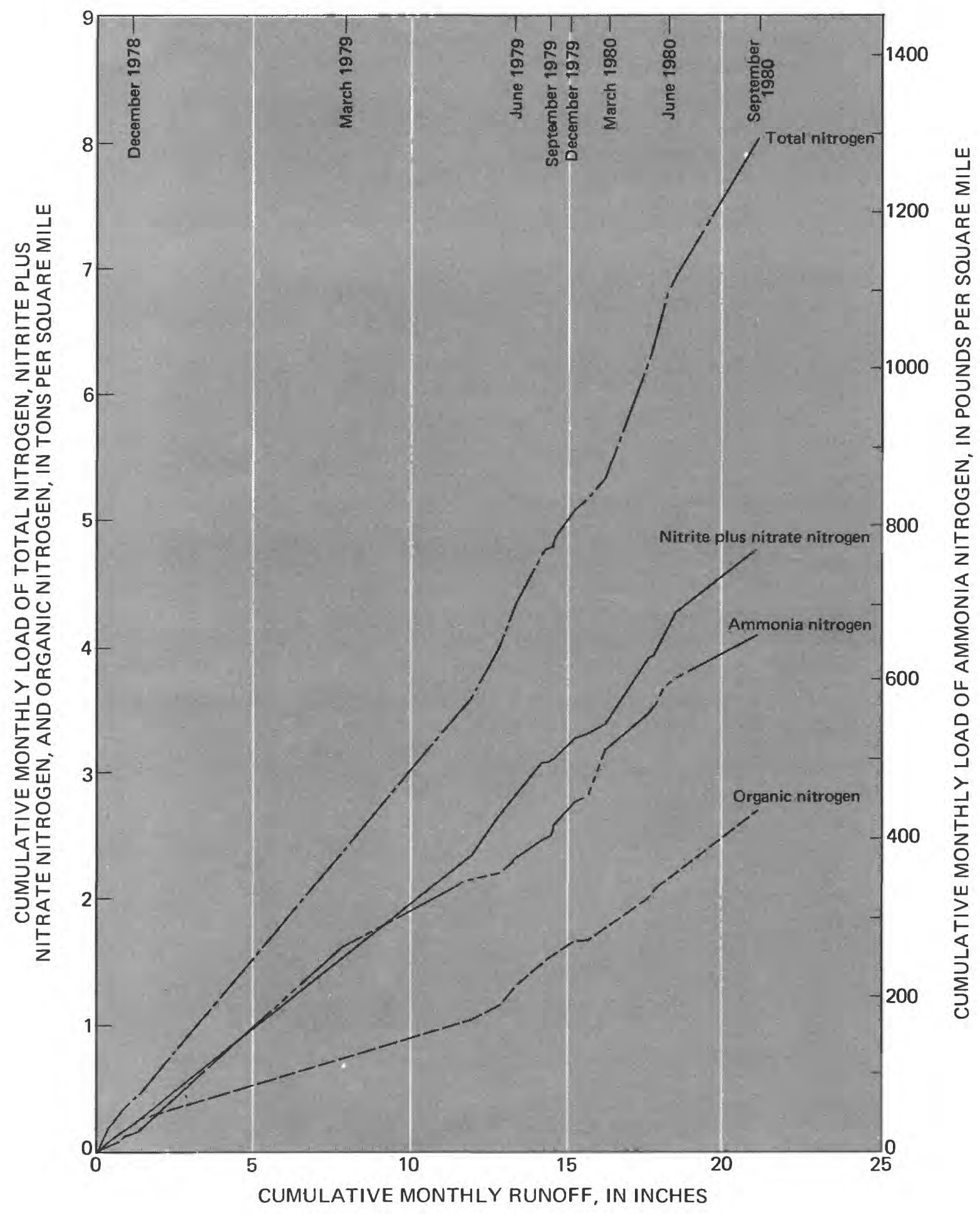

Figure 10. Double mass-accumulation curves of total nitrogen, ammonia nitrogen, nitrite plus nitrate nitrogen, and organic nitrogen as a function of monthly runoff for Onion River downstream of Hingham. 


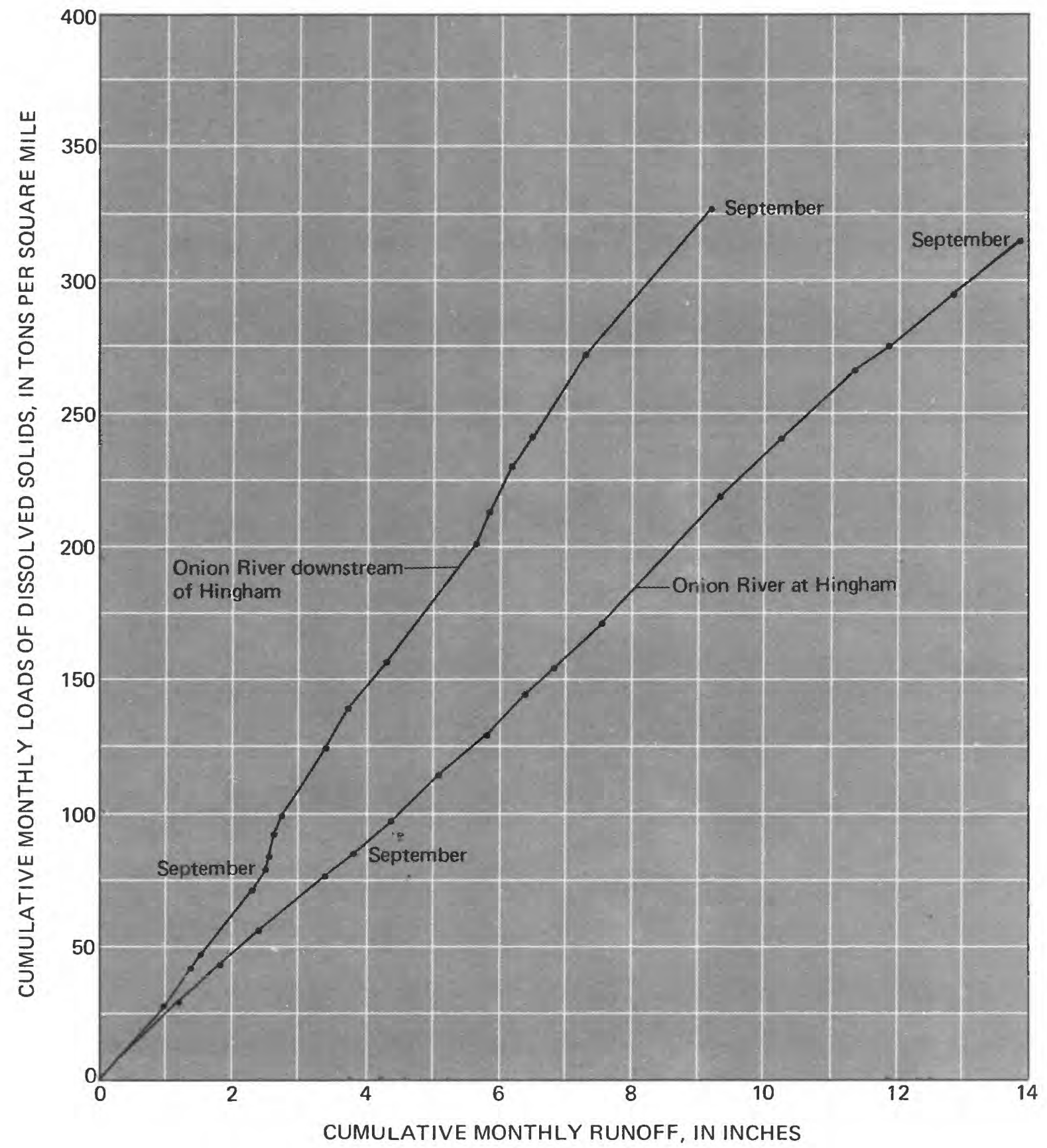

Figure 11. Double mass-accumulation curves of dissolved solids as a function of monthly runoff for Onion River downstream of Hingham. 


\section{Seasonal and Annual Fluctuations}

Seasonal and annual fluctuations in nutrients and sediments were apparent throughout the study. High ammonia concentrations in March 1980 (tables 12 and 13) contrast sharply with the low concentrations in March 1979. The high concentrations in 1980 may be attributed to runoff from manure spread on frozen ground. Snow depths of less than 6 in. in the 1979-80 winter allowed farmers access to the fields to spread manure. During the 1978-79 winter, deep snow (31 in. recorded on January 24 at Sheboygan) may have prevented manure spreading during most of the winter.

Unusually large seasonal fluctuations in concentrations of suspended sediment and total phosphorus during low flow also were noted. The close relationships of phosphorus with suspended sediment have been demonstrated in the Steiner Branch basin (Field and Lidwin, 1982) as well as in other basins (Ward and Eckardt, 1979; McElroy and others, 1976; Verhoff and others, 1979). Some phosphorus sorbs to the soil particles and is transported "piggy-back" with suspended sediments.

To illustrate the seasonal changes in concentrations of suspended-sediment and total phosphorus, average monthly low-flow concentrations are shown in figure 12. The seasonal changes in the concentration for Steiner Branch also are shown for comparison. There is considerable scatter among the data points, and the solid and dashed lines that were drawn are subject to subjective interpretation. However, the purpose of these lines is not to depict absolute values but rather to illustrate the seasonal fluctuations in suspended sediment and phosphorus concentrations in the Onion River. Suspendedsediment concentrations may increase slightly seasonally as shown on the Steiner Branch plot with the phosphorus-concentration curve following the sediment concentration curve. However, the concentrations during summer on Onion River at Hingham and Sheboygan Falls are considerably higher than the authors found in any of the other nonpointsource studies. The higher phosphorus concentrations in summer at Sheboygan Falls may be attributed in part to point sources from Belgium Creek during the canning season, but there are no point sources of contamination above Hingham to cause the high concentrations at that station during summer.

The low points in the suspended sediment and total phosphorus concentration curves coincide with the inactive period of carp because of cold water temperatures. The high point of the curve corresponds to the spawning period when carp are most active. As previously discussed, carp begin spawning at about $15.5^{\circ} \mathrm{C}$ and become most active between $18.5^{\circ}$ and $20^{\circ} \mathrm{C}$. In the spring, these water temperatures were attained at both stations near the end of May and in early June (tables 30 and 31). Therefore, the data suggest that the high concentrations of suspended sediment and total phosphorus during late spring and early summer may be due to carp activity.

Nutrient concentrations during storm runoff at Sheboygan Falls were less than expected, despite the degraded quality of water in the river. The maximum concentrations during storm runoff for suspended sediment, and maximum and median concentrations for total phosphorus, total organic nitrogen, and ammonia nitrogen were generally lower in the Onion River than at Steiner Branch and Bruce Valley Creek; low-flow concentrations were greater (table 9).

Trout require good stream-water quality. Steiner Branch and Bruce Valley Creek can support a trout population (Wisconsin Department of Natural Resources, 1980). In comparison to these streams, storm-runoff concentrations of nutrients and sediments do not seem to be the major factor contributing to the degraded water quality of the Onion River.

\section{SUMMARY}

The U.S. Geological Survey, in cooperation with the Wisconsin Department of Natural Resources, investigated the water quality of the Onion River basin in east-central Wisconsin during the 1979 and 1980 water years. Dairy farming and cash cropping are the major agricultural activities in the basin. Nonpoint-source pollution in the lower part of the Onion River was suspected of contributing significantly to degraded water quality. Two streamflow water-quality-monitoring stations were established on the Onion River; one at Hingham upstream of the problem area and one near Sheboygan Falls downstream from the problem area.

The data-collection program began in December 1978 and ended in September 1980. Its scope included determination of (1) streamflow; (2) the suspended-sediment, nitrogen, and phosphorus loads; (3) water temperature and dissolved solids; and (4) miscellaneous water-quality constituents including dissolved oxygen, $\mathrm{pH}$, biochemical oxy- 

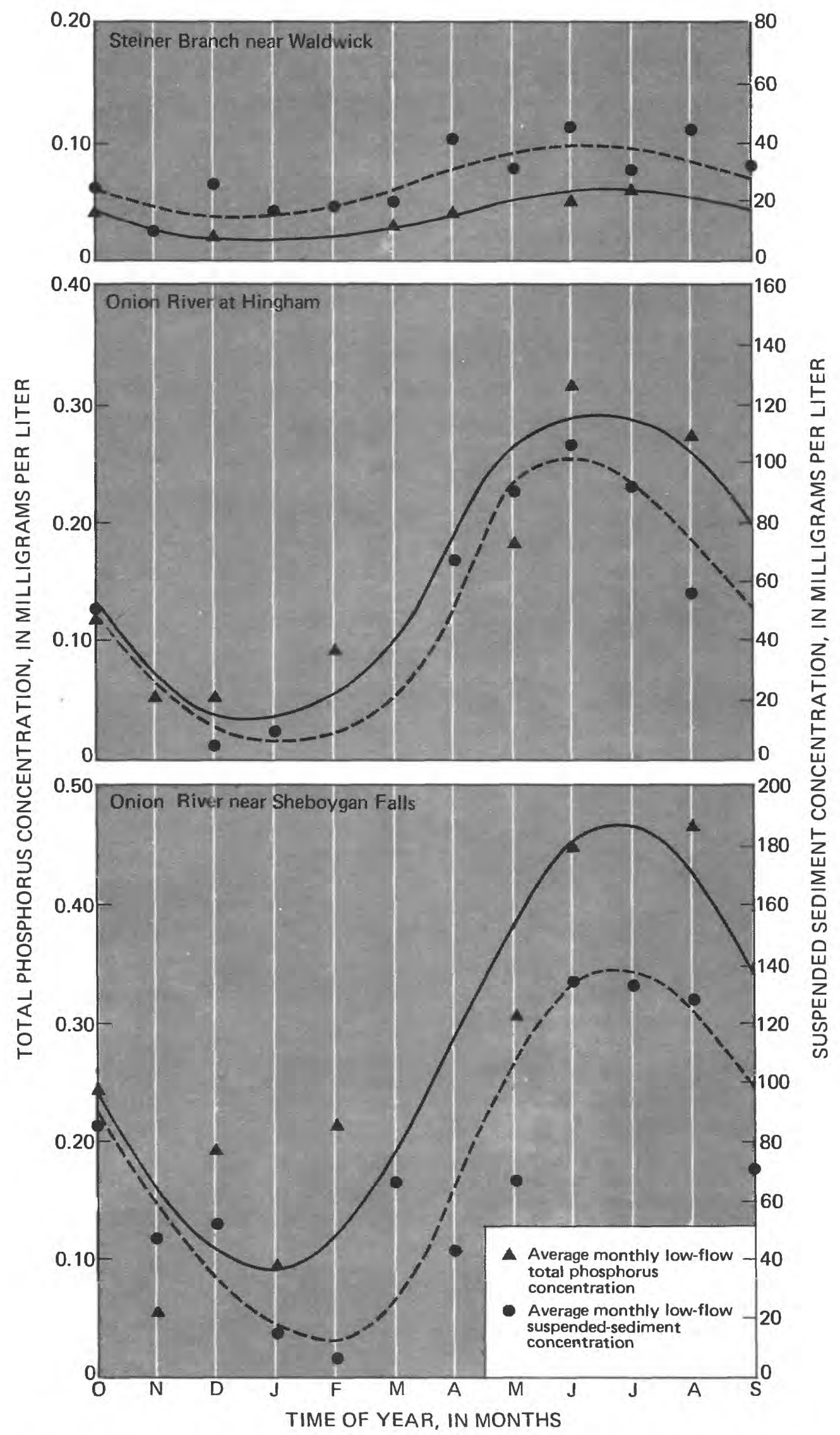

Figure 12. Average monthly suspended-sediment and total phosphorus concentrations during low-flow in the Onion River and Steiner Branch. 
gen demand, fecal coliform and fecal streptococci bacteria, periphyton biomass, pesticides, trace metals, alkalinity, and chloride.

Streamflow at Onion River at Hingham ranged from a minimum 7-day mean flow of 8.7 to a maximum discharge of $600 \mathrm{ft}^{3} / \mathrm{s}$; at Onion River near Sheboygan Falls the range was from a minimum 7-day mean flow of 13 to a maximum discharge of $2,350 \mathrm{ft}^{3} / \mathrm{s}$. Based on discharges at the Sheboygan River at Sheboygan these discharges ranged from about double the low flow that occurs on the average of once every 2 years to discharges of between a 5-year and a 10-year flood frequency. The average discharge at Hingham in the 1979 water year was $32.2 \mathrm{ft}^{3} / \mathrm{s}$ and in the 1980 water year it was $27.6 \mathrm{ft}^{3} / \mathrm{s}$. At Sheboygan Falls the average discharge in the 1979 water year was $94.2 \mathrm{ft}^{3} / \mathrm{s}$ and in the 1980 water year it was $55.2 \mathrm{ft}^{3} / \mathrm{s}$. Based on the average discharge at the Sheboygan River at Sheboygan, the 1979 water year was 60 percent greater than average and the 1980 water year was about 5 percent less than average.

Precipitation for the 1979 water year was 33.1 in. and for the 1980 water year it was 36.8 in.; these amounts are $3.3 \mathrm{in}$. and $7.0 \mathrm{in}$., respectively, above average.

Suspended-sediment yields were greatest at the Sheboygan Falls station but comparison of the double-mass-accumulation curves show loading rates to be about the same for both stations. Suspended-sediment yields were 79.1 ton $/ \mathrm{mi}^{2}$ for the 1979 water year and 63.9 ton $/ \mathrm{mi}^{2}$ for the 1980 water year at Hingham, whereas the yields were 93.5 ton $/ \mathrm{mi}^{2}$ in the 1979 water year and $84.2 \mathrm{ton} / \mathrm{mi}^{2}$ in the 1980 water year downstream of Hingham. Carp resuspend bottom sediments and nutrients causing unusually high concentrations during the summer of both 1979 and 1980.

All nutrient loads were greater in the 1979 water year than in the 1980 water year. The area downstream of Hingham produced higher nutrient yields than the basin upstream of Hingham. In addition, double-mass-accumulation curves indicated highest loading rates downstream of Hingham. These higher loading rates may be due to the impact of point sources on Belgium Creek. The data on pointsource loading are rather limited, however, and warrant further investigation. Phosphorus yields were $331 \mathrm{lb} / \mathrm{mi}^{2}$ for the 1979 water year and 317 $\mathrm{lb} / \mathrm{mi}^{2}$ for the 1980 water year at Hingham; downstream of Hingham the yields were $656 \mathrm{lb} / \mathrm{mi}^{2}$ for the 1979 water year and $647 \mathrm{lb} / \mathrm{mi}^{2}$ for the 1980 water year. During the 1979 water year downstream of Hingham, 45 percent of the total phosphorus load was attributed to point sources of contamination. Ammonia concentrations at both stations did not exceed the DNR criterion of $0.04 \mathrm{mg} / \mathrm{L}$ of un-ionized ammonia $\left(\mathrm{NH}_{3}-\mathrm{N}\right)$. Most phosphorus concentrations during base flow and surface runoff exceeded the U.S. EPA criterion of $0.10 \mathrm{mg} / \mathrm{L}$ for prevention of biological nusiance growths.

\section{SELECTED REFERENCES}

Burns, J. W., 1966, Carp, in Reproductive behavior of carp, compiled by Calhoun, Alex, 1966: California Department of Fish and Game, Inland Fisheries Management, $546 \mathrm{p}$.

Conger, D. H., 1981, Techniques for estimating magnitude and frequency of floods for Wisconsin streams: U.S. Geological Survey WaterResources Investigations Open-File Report $80-1214,116 \mathrm{p}$.

Dill, W. A., and Wales, J. H., 1945, The fishery of Shasta Lake, Shasta County: California Department of Fish and Game, Inland Fisheries Administration, Report No. 1.A, preliminary account, Report 045-8, 149 p.

Donigan, A. S., Jr., and Crawford, N. H., 1976, Modeling nonpoint pollution from the land surface: U.S. Environmental Protection Agency, EPA-6003-76-083, 279 p.

Engel, R. J., Roberts, B. A., Steingraeber, J. A., 1978, Soil survey of Sheboygan County, Wisconsin: U.S. Department of Agriculture Soil Conservation Service, $116 \mathrm{p}$.

Environmental Data Service, 1973, Monthly normals of temperature, precipitation and heating and cooling degree days for Wisconsin: Washington, D.C., U.S. Department of Commerce, Climatography of the United States, No. 81.

Field, S. J., and Lidwin, R. A., 1982, Water quality assessment of Steiner Branch basin, Lafayette County, Wisconsin: U.S. Geological Survey Water-Resources Investigations 81-52, $58 \mathrm{p}$.

Greeley, J. R., 1927, Fishes of the Oswego watershed: New York Conservation Department, Annual report, Supplement 17, 93 p.

Guy, P., and Norman, V. W., 1970, Field methods for measurement of fluvial sediment: U.S. Geological Survey Techniques of WaterResources Investigations, Book 3, Chapter C2, $50 \mathrm{p}$.

Hindall, S. M., 1975, Measurement and prediction of sediment yields in Wisconsin streams: U.S. Geological Survey Water-Resources Investigations 54-75, $27 \mathrm{p}$.

Holmstrom, B. K., 1979, Low-flow characteristics of Wisconsin streams at sewage-treatment 
plants and industrial plants: U.S. Geological Survey Water-Resources Investigations 79-31, $123 \mathrm{p}$.

Linsley, R. K. Jr., Kohler, M. A., Paulhus, J. L. H., 1975, Hydrology for engineers (2d ed.): New York, McGraw-Hill Book Company, 482 p.

McElroy, A. D., Chiu, S. Y., Nebgen, J. W., Aleti, A., and Bennett, F. W., 1976, Loading functions for assessment of water pollution from nonpoint sources: Midwest Research Institute, Kansas City, Mo., U.S. Environmental Protection Agency Report EPA-600-2-76-151, 444 p.

Parker, D. E., Kurer, D. C., Steingraeber, J. A., 1970, Soil Survey of Ozaukee County, Wisconsin: U.S. Department of Agriculture Soil Conservation Service, $92 \mathrm{p}$.

Porterfield, George, 1972, Computation of fluvialsediment discharge: U.S. Geological Survey Techniques of Water Resources Investigations, Book 3, Chapter C3, 66 p.

Sigler, W. F., 1958, The ecology and use of carp in Utah: Utah Agricultural Experimental Station, Bulletin 405, $63 \mathrm{p}$.

U.S. Department of Commerce, 1979, Climatological data, Wisconsin: U.S. Department of Commerce, v. 84 , no. 3,18 p.

1980, Climatological data, Wisconsin: U.S. Department of Commerce, v. 85, no. 3, 18 p.

U.S. Congress, 1972, Federal water pollution control act amendments of 1972, Public Law 92-500, section 208: U.S. 92d Congress, 2d session, p. 25-26.

U.S. Environmental Protection Agency, 1977 [1978], Quality criteria for water, 1976: U.S. Environmental Protection Agency, Washington, D.C., $256 \mathrm{p}$.
U.S. Geological Survey, 1980, Water resources data for Wisconsin, 1979: U.S. Geological Survey Water-Data Report WI-79-1, 514 p.

1981, Water resources data for Wisconsin, 1980: U.S. Geological Survey Water Data Report WI-80-1, $505 \mathrm{p}$.

Verhoff, F. G., Melfi, D. A., and Yalesick, S. M., 1979, Storm travel distance calculations for total phosphorus and suspended material in rivers: Water Resources Research, v. 15, no. 6, p. 1,354-1,360.

Wales, J. H., 1943, Report of investigation on Clear Lake, Lake County: U.S. Department of Fish and Game, Inland Fishery Administration, Report No. 43-4, 30 p.

Ward, J. R., and Eckhardt, D. A., 1979, Nonpoint-source discharges in Pequea Creek basin, Pennsylvania, 1977: U.S. Geological Survey Water-Resources Investigations Report 79-88, $110 \mathrm{p}$.

Wisconsin Department of Agriculture, 1961, Wisconsin climatological data: Madison, Wisconsin Crop Reporting Service Publication, 168 p.

Wisconsin Department of Natural Resources, 1972, Wisconsin lakes: Madison, Wisconsin Department of Natural Resources Publication 218-72, $79 \mathrm{p}$.

1976, DNR information paper on nonpoint-source pollution: Madison, Wisconsin Department of Natural Resources, $5 \mathrm{p}$.

1979, Sheboygan River assessment reports: Madison, Wisconsin Department of Natural Resources, $165 \mathrm{p}$.

1980, Wisconsin trout streams: Madison, Wisconsin Department of Natural Resources, $67 \mathrm{p}$.

1981, The Onion River priority watershed plan: Madison, Wisconsin Department of Natural Resources, $31 \mathrm{p}$. 
Table 1. Percentage of land use in the Onion River basin

[Data, in percentage of total area, from J. S. Bauman (Wisconsin Department of Natural Resources, written commun., 1982)]

\begin{tabular}{lcc}
\hline Use & $\begin{array}{c}\text { Upstream of } \\
\text { Hingham dam }\end{array}$ & $\begin{array}{c}\text { Downstream of } \\
\text { Hingham dam }\end{array}$ \\
\hline Cropland & 71 & 89 \\
Pasture & 1 & 1 \\
Woodland & 22 & 6 \\
Wetlands & 4 & 3 \\
Urban & 1 & 1 \\
Other & 1 & 0 \\
\hline
\end{tabular}

Table 2. Summary of streamflow characteristics for Onion River at Hingham, Onion River near Sheboygan Falls, and Sheboygan River at Sheboygan, 1979 and 1980 water years

\begin{tabular}{|c|c|c|c|c|c|c|}
\hline \multirow[t]{2}{*}{ Streamf low characteristic } & \multicolumn{2}{|c|}{$\begin{array}{l}\text { Onion River } \\
\text { at Hingham }\end{array}$} & \multicolumn{2}{|c|}{$\begin{array}{l}\text { Onton River near } \\
\text { Sheboygan Falls }\end{array}$} & \multicolumn{2}{|c|}{$\begin{array}{l}\text { Sheboygan River } \\
\text { at Sheboygan }\end{array}$} \\
\hline & 1979 & 1980 & 1979 & 1980 & 1979 & 1980 \\
\hline $\begin{array}{l}\text { Total discharge, in fublc } \\
\text { feet per second-days }\end{array}$ & 11,747 & 10,097 & 33,717 & 20,193 & 141,421 & 83,986 \\
\hline $\begin{array}{l}\text { Mean discharge, in cubic } \\
\text { feet per second. }\end{array}$ & 32.2 & 27.6 & 92.4 & 55.2 & 387 & 229 \\
\hline $\begin{array}{l}\text { Mean discharge, in } \\
\text { cuble feet per second } \\
\text { per square mile. }\end{array}$ & 0.87 & 0.74 & 0.98 & 0.59 & 0.93 & 0.55 \\
\hline Runoff, In Inches & 11.75 & 10.10 & 13.33 & 7.98 & 12.59 & 7.47 \\
\hline $\begin{array}{l}\text { Minimum 7-day mean low } \\
\text { flow, in cubic feet per } \\
\text { second. }\end{array}$ & 11 & 8.7 & 14 & 13 & 47 & 52 \\
\hline $\begin{array}{l}\text { Maximum recorded peak } \\
\text { discharge, in cubic feet } \\
\text { per second. }\end{array}$ & 600 & 407 & 2,350 & 774 & 6,460 & 2,540 \\
\hline
\end{tabular}

1 The sum of the dally mean discharges for the entire water year.

Table 3. Precipitation and runoff for Onion River near Sheboygan Falls for selected periods

\begin{tabular}{|c|c|c|c|c|c|c|}
\hline & 1979 & \multicolumn{2}{|l|}{ water year } & \multicolumn{3}{|c|}{1980 water year } \\
\hline & Nov.-Apri1 & May-Sept. & Total & Nov.-Apr11 & May-Sept. & Total \\
\hline $\begin{array}{l}\text { Precipitation } \\
\text { (1nches). }\end{array}$ & 16.2 & 17.0 & 33.2 & 10.1 & 26.7 & 36.8 \\
\hline Runoff (inches) & 9.79 & 3.54 & 13.3 & 3.89 & 4.09 & 7.98 \\
\hline
\end{tabular}


Table 4. Base flow and storm runoff for Onion River at Hingham and

Onion River at Sheboygan Falls, 1979 and 1980 water years

\begin{tabular}{llllllll}
\hline & \multicolumn{3}{c}{ Onion River at Hingham } & & Onion Rfver near Sheboygan Falls \\
\cline { 2 - 5 } $\begin{array}{l}\text { Water } \\
\text { year }\end{array}$ & Base flow & $\begin{array}{c}\text { Surface } \\
\text { runoff } \\
\left(\mathrm{ft}^{3} / \mathrm{s}\right)\end{array}$ & $\begin{array}{c}\text { Surface runoff } \\
\text { as a percentage } \\
\text { of base flow }\end{array}$ & & Base flow & $\begin{array}{c}\text { Surface } \\
\text { runoff } \\
\left(\mathrm{ft}^{3} / \mathrm{ft}^{3} / \mathrm{s}\right)\end{array}$ & $\begin{array}{c}\text { Surface runoff } \\
\text { as a percentage } \\
\text { of base flow }\end{array}$ \\
\hline 1979 & 6,620 & 5,130 & 56 & & 11,600 & 22,100 & 34 \\
1980 & 5,020 & 5,070 & 50 & 8,750 & 11,400 & 43 \\
\hline
\end{tabular}

Table 5. Comparison of base-flow discharges at Hingham, Gibbsville, and Sheboygan Falls [Adapted from Holmstrom, 1979. ---, no measurements made.]

\begin{tabular}{|c|c|c|c|}
\hline Date of measurement & Hingham & Gibbsville & Sheboygan Falls \\
\hline Drainage area, $\mathrm{ml}^{2}$ & 36.2 & 82.0 & 94.1 \\
\hline July 11,1973 & 8.35 & --- & --- \\
\hline July 12,1973 & -- & 11.3 & 8.45 \\
\hline Aug. 12, 1975 & 5.62 & --- & --- \\
\hline Aug. 13, 1975 & -- & 12.2 & 5.58 \\
\hline Aug. 10, 1976 & 9.06 & 10.6 & 9.15 \\
\hline July 12, 1977 & 8.51 & 8.38 & 8.64 \\
\hline
\end{tabular}

Table 6. Concentrations of nutrients in base-flow samples from the Onion River at Hingham and Sheboygan Falls [Data in millgrams per 11ter]

\begin{tabular}{|c|c|c|c|c|c|c|c|c|c|c|c|c|c|c|}
\hline 1979 & \multicolumn{2}{|c|}{$\begin{array}{r}\text { Nitrite } \\
\text { nitrogen }\end{array}$} & \multicolumn{2}{|c|}{$\begin{array}{c}\text { Nitrite plus } \\
\text { nitrate nitrogen }\end{array}$} & \multicolumn{2}{|c|}{$\begin{array}{r}\text { Ammonia } \\
\text { nitrogen }\end{array}$} & \multicolumn{2}{|c|}{$\begin{array}{r}\text { Organic } \\
\text { nitrogen }\end{array}$} & \multicolumn{2}{|c|}{$\begin{array}{l}\text { Total } \\
\text { nitrogen }\end{array}$} & \multicolumn{2}{|c|}{$\begin{array}{c}\text { Total } \\
\text { phosphorus }\end{array}$} & \multicolumn{2}{|c|}{$\begin{array}{l}\text { Orthophosphate } \\
\text { phosphorus }\end{array}$} \\
\hline June $\mathbf{3 8}$ & 0.05 & 0.01 & 0.59 & 0.09 & 0.20 & 0.23 & 1.5 & 2.4 & 2.3 & 2.6 & 0.31 & 0.44 & 0.11 & 0.15 \\
\hline August 8 & .01 & .03 & .17 & .43 & .14 & .11 & .78 & .88 & 1.1 & 1.4 & .19 & .40 & .06 & .15 \\
\hline December 14 & .01 & $<.01$ & 1.6 & 1.2 & .05 & .08 & .54 & .51 & 2.2 & 1.8 & .05 & .13 & .05 & .09 \\
\hline$\underline{1980}$ & & & & & & & & & & & & & & \\
\hline May 8 & .02 & .02 & .41 & .03 & .31 & .36 & 1.5 & 1.7 & 2.2 & 2.1 & .17 & .40 & .02 & .03 \\
\hline October 7 & .01 & $1-$ & 1.4 & 1.5 & $<.01$ & .02 & .67 & 1.1 & 2.1 & 2.6 & .12 & .24 & .06 & .12 \\
\hline Mean & .02 & .02 & .83 & .65 & .14 & .16 & 1.0 & 1.3 & 2.0 & 2.1 & .17 & .32 & .06 & .11 \\
\hline
\end{tabular}

1 --, no sample collected. 
Table 7. Constituent loads and percentage of annual load for storms sampled

\begin{tabular}{|c|c|c|c|c|c|c|}
\hline \multirow{2}{*}{$\begin{array}{l}\text { Dates } \\
\text { of storms }\end{array}$} & \multicolumn{2}{|c|}{ Total nitrogen } & \multicolumn{2}{|c|}{$\begin{array}{c}\text { Nitrite plus nitrate } \\
\text { nitrogen }\end{array}$} & \multicolumn{2}{|c|}{ Organic nitrogen } \\
\hline & $\begin{array}{l}\text { Load } \\
\text { (1b) }\end{array}$ & $\begin{array}{l}\text { Percentage of } \\
\text { annual load }\end{array}$ & $\begin{array}{l}\text { Load } \\
(1 b)\end{array}$ & $\begin{array}{l}\text { Percentage of } \\
\text { annual load }\end{array}$ & $\begin{array}{l}\text { Load } \\
\text { (1b) }\end{array}$ & $\begin{array}{l}\text { Percentage of } \\
\text { annual load }\end{array}$ \\
\hline \multicolumn{7}{|c|}{ Onion River at Hingham } \\
\hline March 19-24 & 31,900 & 18.1 & 23,600 & 22.4 & 7,640 & 12.7 \\
\hline March 30 to April 1 & 17,100 & 9.7 & 10,300 & 9.8 & 6,360 & 10.6 \\
\hline June 28 to July 1 & 2,220 & 1.3 & 1,020 & 1.0 & 1,100 & 1.8 \\
\hline August $8-12$ & 5,160 & 2.9 & 1,780 & 1.7 & 3,080 & 5.1 \\
\hline August $20-22$ & 1,620 & .9 & 662 & .6 & 1,050 & 1.7 \\
\hline \multicolumn{7}{|l|}{$\underline{1980}$} \\
\hline January $17-24$ & 4,950 & 3.8 & 2,200 & 3.2 & 2,290 & 3.8 \\
\hline March 17-18 & 1,450 & 1.1 & 530 & .8 & 675 & 1.1 \\
\hline Apri1 8-10 & 10,400 & 8.0 & 6,730 & 9.9 & 3,330 & 5.6 \\
\hline June 5-12 & 8,590 & 6.6 & 3,330 & 4.9 & 5,060 & 8.5 \\
\hline July 20-22 & 2,700 & 2.1 & 1,060 & 1.6 & 1,440 & 2.4 \\
\hline August $8-11$ & 3,170 & 2.4 & 1,470 & 2.2 & 1,700 & 2.9 \\
\hline September $22-25$ & 6,940 & 5.3 & 3,110 & 4.6 & 3,560 & 6.0 \\
\hline \multicolumn{7}{|c|}{ Onion River near Sheboygan Falls } \\
\hline \multicolumn{7}{|l|}{1979} \\
\hline March 19-24 & 166,000 & 22.9 & 116,000 & 25.3 & 41,500 & 17.8 \\
\hline March 30 to April 1 & 73,500 & 10.2 & 46,900 & 10.2 & 22,300 & 9.6 \\
\hline June 28 to July 1 & 9,970 & 1.4 & 6,200 & 1.4 & 3,690 & 1.6 \\
\hline August $8-12$ & 22,800 & 3.2 & 12,000 & 2.6 & 10,300 & 4.4 \\
\hline August $20-22$ & 7,870 & 1.1 & 3,820 & .8 & 3,680 & 1.6 \\
\hline August 25 & 4,610 & .6 & 2,220 & .5 & 2,100 & .9 \\
\hline \multicolumn{7}{|l|}{1980} \\
\hline January $16-19$ & 15,900 & 3.2 & 11,000 & 4.2 & 9,340 & 4.8 \\
\hline March 18-21 & 14,300 & 2.8 & 5,330 & 2.0 & 6,190 & 3.2 \\
\hline Apr11 5-10 & 76,600 & 15.2 & 57,900 & 21.9 & 18,500 & 9.5 \\
\hline June 5-12 & 43,800 & 8.7 & 25,700 & 9.7 & 14,500 & 7.5 \\
\hline Ju1y 20-22 & 21,100 & 4.2 & 12,400 & 4.7 & 7,360 & 3.8 \\
\hline August $8-11$ & 24,500 & 4.9 & 13,100 & 4.9 & 9,740 & 5.0 \\
\hline September $17-30$ & 89,500 & 17.7 & 53,300 & 20.2 & 36,200 & 18.6 \\
\hline
\end{tabular}


Table 7. Constituent loads and percentage of annual load for storms sampled--Continued

\begin{tabular}{lllll}
\hline Ammonia nitrogen & Total phosphorus & $\begin{array}{c}\text { Orthophosphate } \\
\text { phosphorus }\end{array}$ & Suspended sediment \\
\hline $\begin{array}{l}\text { Load Percentage of } \\
\text { (1b) annual load }\end{array}$ & $\begin{array}{c}\text { Load Percentage of } \\
\text { (1b) }\end{array} \begin{array}{c}\text { Load Percentage of } \\
\text { annal load }\end{array}$ & $\begin{array}{c}\text { Load Percentage of } \\
\text { (1b) }\end{array}$ \\
\hline
\end{tabular}

Onion River at Hingham

$\begin{array}{rrrrrrrr}951 & 12.8 & 1,430 & 11.7 & 879 & 16.7 & 182 & 6.2 \\ 678 & 9.2 & 1,540 & 12.5 & 637 & 12.1 & 483 & 16.4 \\ 116 & 1.6 & 239 & 1.9 & 84 & 1.6 & 79.2 & 2.7 \\ 301 & 4.1 & 717 & 5.8 & 314 & 6.0 & 176 & 6.0 \\ 82 & 1.1 & 213 & 1.7 & 78 & 1.5 & 29.9 & 1.0\end{array}$

$\begin{array}{rrrrrrrr}278 & 4.5 & 485 & 4.1 & 193 & 3.7 & 24.9 & 1.0 \\ 244 & 3.9 & 164 & 1.4 & 117 & 2.2 & 5.6 & .2 \\ 339 & 5.5 & 767 & 6.5 & 234 & 4.5 & 176 & 7.4 \\ 374 & 6.0 & 955 & 8.1 & 291 & 5.6 & 234 & 9.8 \\ 96 & 1.5 & 393 & 3.3 & 148 & 2.8 & 96.0 & 4.0 \\ 292 & 4.7 & 532 & 4.5 & 457 & 8.7 & 95.9 & 4.0 \\ 254 & 4.1 & 1,010 & 8.6 & 432 & 8.3 & 251 & 10.6\end{array}$

Onion River near Sheboygan Fa11s

\begin{tabular}{rrrrrrrr}
8,490 & 28.2 & 8,520 & 17.2 & 5,340 & 22.3 & 1,300 & 15.8 \\
4,110 & 13.7 & 5,600 & 11.3 & 2,780 & 11.6 & 1,710 & 20.7 \\
244 & .8 & 728 & 1.5 & 255 & 1.1 & 140 & 1.7 \\
607 & 2.0 & 2,570 & 5.2 & 1,030 & 4.3 & 581 & 7.0 \\
181 & .6 & 858 & 1.7 & 299 & 1.2 & 133 & 1.6 \\
120 & .4 & 490 & 1.0 & 188 & .8 & 72 & .9 \\
1,060 & 5.2 & 2,280 & 4.7 & 1,220 & 5.5 & 145 & 2.0 \\
2,890 & 14.0 & 1,570 & 3.2 & 1,120 & 5.0 & 53.2 & .7 \\
1,380 & 6.7 & 3,600 & 7.4 & 1,200 & 5.4 & 587 & 8.2 \\
939 & 4.5 & 3,260 & 6.7 & 1,090 & 4.9 & 917 & 12.8 \\
426 & 2.1 & 1,870 & 3.9 & 696 & 3.1 & 511 & 7.1 \\
794 & 3.8 & 2,660 & 5.5 & 1,600 & 7.2 & 517 & 7.2 \\
2,260 & 10.9 & 11,600 & 23.9 & 6,110 & 27.3 & 1,970 & 27.5 \\
\hline
\end{tabular}


Table 8. Loads and yields of chemical constituents in the Onion River basin, 1979 and 1980 water years

\begin{tabular}{|c|c|c|c|c|c|c|c|c|c|}
\hline \multirow[t]{2}{*}{ Const1tuent } & \multicolumn{3}{|c|}{$\begin{array}{l}\text { Onfon River near Hingham } \\
\text { (Drainage area }=37.2 \mathrm{mi}^{2} \text { ) }\end{array}$} & \multicolumn{3}{|c|}{$\begin{array}{l}\text { Onton R1 ver near Sheboygan Falls } \\
\text { (Drainage area }=94.1 \mathrm{mi}^{2} \text { ) }\end{array}$} & \multicolumn{3}{|c|}{$\begin{array}{l}\text { Onton River downstream } \\
\text { from Hingham } \\
\text { (Drainage area }=56.9 \mathrm{mi}^{2} \text { ) }\end{array}$} \\
\hline & Pounds : & $\begin{array}{l}\text { Pounds per } \\
\text { square mile }\end{array}$ & $\begin{array}{l}\text { Pounds per } \\
\text { square mile } \\
\text { per day }\end{array}$ & Pounds & $\begin{array}{l}\text { Pounds per } \\
\text { square mila }\end{array}$ & $\begin{array}{l}\text { Pounds per } \\
\text { square mile } \\
\text { per day }\end{array}$ & Pounds & $\begin{array}{l}\text { Pounds per } \\
\text { square wile }\end{array}$ & $\begin{array}{c}\text { Pounds per } \\
\text { square mile } \\
\text { per day }\end{array}$ \\
\hline \multicolumn{10}{|c|}{1979 water year } \\
\hline Total nitrogen & 176,000 & 4,740 & 13.0 & 723,000 & 7,680 & 21.0 & 546,000 & 9,600 & 26.3 \\
\hline Organic nitrogen & 60,100 & 1,620 & 4.43 & 233,000 & 2,470 & 6.78 & 173,000 & 3,040 & 8.32 \\
\hline Ammonia nitrogau & 7,400 & 199 & .54 & 30,100 & 320 & .88 & 22,700 & 399 & 1.09 \\
\hline Nitrite nitrate & 106,000 & 2,840 & 7.79. & 458,000 & 4,860 & 13.3 & 352,000 & 6,180 & 16.9 \\
\hline Phosphorus & 12,300 & 331 & .91 & 49,600 & 528 & 1.45 & 37,300 & 656 & 1.80 \\
\hline $\begin{array}{l}\text { Orthophosphate } \\
\text { phosphorus }\end{array}$ & 5,280 & 142 & .39 & 24,000 & 254 & .70 & 18,700 & 329 & .91 \\
\hline $\begin{array}{l}\text { Total suspended- } \\
\text { sadiment yields } \\
\text { In tonnage } \\
\text { equivalents. }\end{array}$ & $\begin{array}{l}2,940 \\
\text { tons }\end{array}$ & $\begin{array}{l}79 \cdot 1 \\
\tan / \mathrm{mi}^{2}\end{array}$ & $\left.\underset{\left(\operatorname{ton} / \mathrm{mI}^{2}\right.}{.22}\right)^{/ \mathrm{d}}$ & $\begin{array}{l}8,260 \\
\text { tons }\end{array}$ & $\begin{array}{r}87 \cdot 8 \\
\operatorname{ton} / m 1^{2}\end{array}$ & $\left(\operatorname{ton} / m 1^{2}\right) / d$ & $\begin{array}{l}5,320 \\
\text { tons }\end{array}$ & $\begin{array}{r}93.5 \\
\operatorname{ton} / \mathrm{mi}^{2}\end{array}$ & $\left(\operatorname{ton} / \mathrm{mi}^{2}\right) / \mathrm{d}$ \\
\hline \multicolumn{10}{|l|}{$\underline{1980}$ watar year } \\
\hline Total nitrogen & 131,000 & 3,520 & 9.63 & 505,000 & 5,360 & 14.7 & 373,000 & 6,560 & 18.0 \\
\hline Organic nitrogen & 59,500 & 1,600 & 4.37 & 194,000 & 2,060 & 5.63 & 135,000 & 2,360 & 6.46 \\
\hline Ammonia nitrogen & 6,190 & 166 & .45 & 20,600 & 219 & .60 & 14,400 & 254 & .69 \\
\hline Nitrite nitrate & 68,100 & 1,830 & 5.00 & 265,000 & 2,810 & 7.68 & 197,000 & 3,450 & 9.44 \\
\hline Phosphorus & 11,800 & 317 & .87 & 48,700 & 517 & 1.41 & 36,800 & 647 & 1.77 \\
\hline $\begin{array}{l}\text { Orthophosphate } \\
\text { phosphorus }\end{array}$ & 5,220 & 140 & .38 & 22,400 & 238 & .65 & 17,100 & 301 & .82 \\
\hline $\begin{array}{l}\text { Total suspendad- } \\
\text { sadiment ylelds } \\
\text { in tonnage aquivalents. }\end{array}$ & $\begin{array}{l}2,380 \\
\text { tons }\end{array}$ & $\begin{array}{r}63.9 \\
\operatorname{ton} / \mathrm{mi}^{2}\end{array}$ & $\left(\operatorname{ton} / \mathrm{mi}^{2}\right) / \mathrm{d}$ & $\begin{array}{l}7,170 \\
\text { tons }\end{array}$ & ${ }_{\text {ton } / m 1^{2}}{ }^{76.3}$ & $\underset{\left(\operatorname{ton} / \mathrm{mt}^{2}\right.}{.21}$ )/d & $\begin{array}{l}4,800 \\
\text { tons }\end{array}$ & $\begin{array}{r}84 \cdot \frac{2}{2} \\
\text { ton } / \mathrm{mit}^{2}\end{array}$ & $\left(\operatorname{ton} / \mathrm{mt}^{2}\right) / \mathrm{d}$ \\
\hline
\end{tabular}

Table 9. Suspended-sediment and nutrient concentrations during low-flow and storm-runoff periods in the Onion River. Steiner Branch, and Bruce Valley Creek

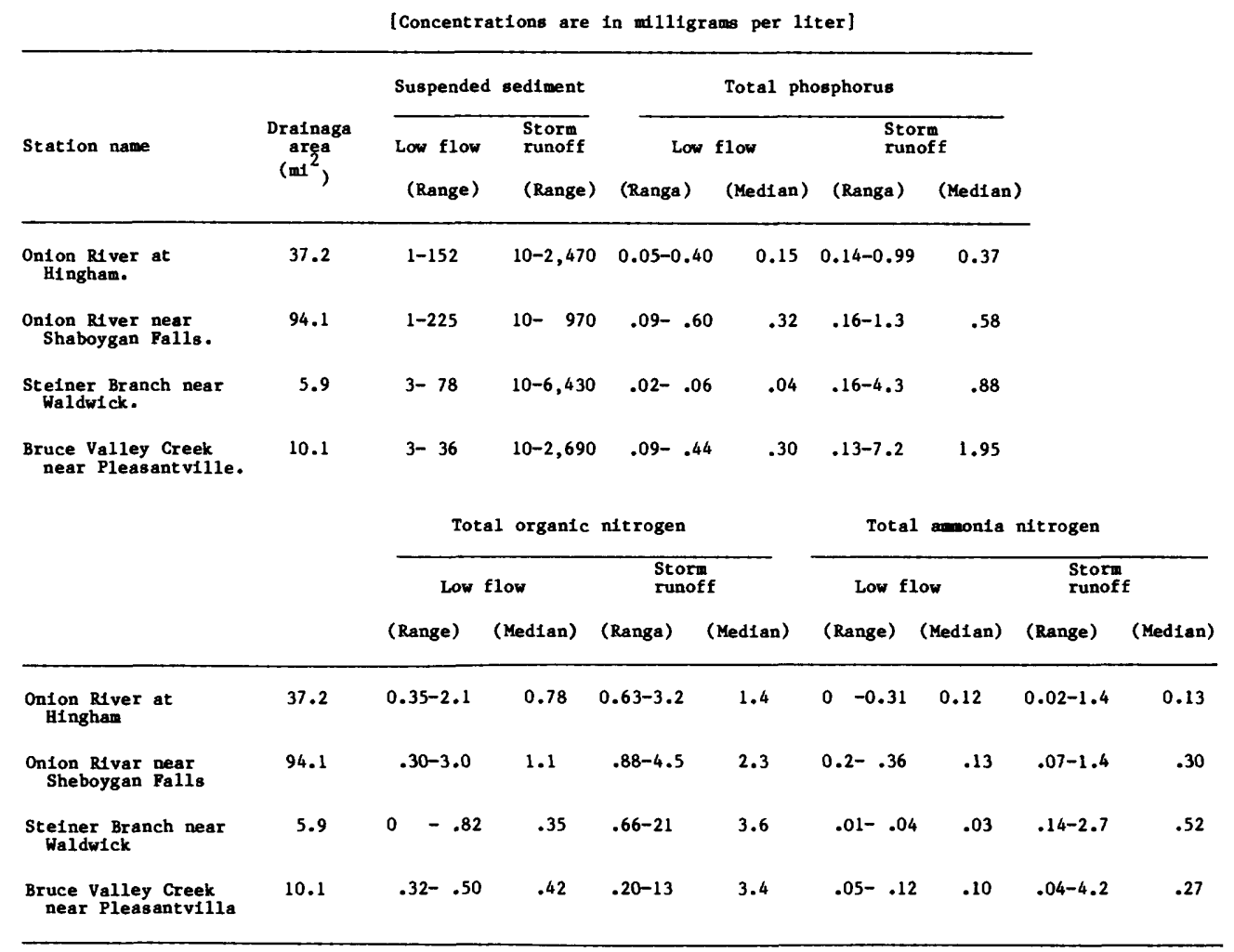


Table 10. Stream discharges for Onion River at Hingham, 1979 and 1980 water years

\begin{tabular}{|c|c|c|c|c|c|c|c|c|c|c|c|c|}
\hline \multirow[b]{2}{*}{ DAY } & \multirow[b]{2}{*}{ ocr } & \multicolumn{2}{|c|}{ DISCHARGE, I } & \multirow{2}{*}{$\begin{array}{c}\text { CUBIC FEE? } \\
\text { JAN }\end{array}$} & \multicolumn{2}{|c|}{ QT PER SECOND WATER } & \multirow{2}{*}{$\begin{array}{l}\text { YEAR } \\
\text { APR }\end{array}$} & \multirow{2}{*}{$\begin{array}{r}\text { YTOBER } 1 \\
\text { MAY }\end{array}$} & \multicolumn{2}{|c|}{ B TO SEPTEMBER } & \multirow[b]{2}{*}{ AUG } & \multirow[b]{2}{*}{ SEF } \\
\hline & & Nov & DEC & & FEB & MAR & & & JUN & JUL & & \\
\hline $\begin{array}{l}1 \\
2 \\
3 \\
4 \\
5\end{array}$ & $\begin{array}{l}34 \\
32 \\
30 \\
28 \\
27\end{array}$ & $\begin{array}{l}11 \\
11 \\
11 \\
11 \\
11\end{array}$ & $\begin{array}{l}24 \\
23 \\
22 \\
21 \\
20\end{array}$ & $\begin{array}{l}14 \\
14 \\
14 \\
13 \\
13\end{array}$ & $\begin{array}{l}11 \\
11 \\
11 \\
11 \\
11\end{array}$ & $\begin{array}{l}19 \\
20 \\
21 \\
22 \\
23\end{array}$ & $\begin{array}{l}165 \\
100 \\
85 \\
104 \\
120\end{array}$ & $\begin{array}{l}68 \\
61 \\
99 \\
82 \\
55\end{array}$ & $\begin{array}{l}23 \\
20 \\
18 \\
23 \\
22\end{array}$ & $\begin{array}{l}45 \\
38 \\
24 \\
25 \\
25\end{array}$ & $\begin{array}{l}16 \\
15 \\
14 \\
14 \\
21\end{array}$ & $\begin{array}{l}18 \\
18 \\
15 \\
14 \\
14\end{array}$ \\
\hline $\begin{array}{r}6 \\
7 \\
8 \\
9 \\
10\end{array}$ & $\begin{array}{l}37 \\
33 \\
30 \\
29 \\
27\end{array}$ & $\begin{array}{l}11 \\
12 \\
12 \\
12 \\
13\end{array}$ & $\begin{array}{l}20 \\
20 \\
20 \\
19 \\
18\end{array}$ & $\begin{array}{l}13 \\
13 \\
13 \\
13 \\
13\end{array}$ & $\begin{array}{l}11 \\
11 \\
12 \\
12 \\
12\end{array}$ & $\begin{array}{l}24 \\
24 \\
24 \\
23 \\
23\end{array}$ & $\begin{array}{r}102 \\
67 \\
56 \\
52 \\
52\end{array}$ & $\begin{array}{l}47 \\
42 \\
38 \\
35 \\
32\end{array}$ & $\begin{array}{l}21 \\
26 \\
22 \\
25 \\
39\end{array}$ & $\begin{array}{l}20 \\
17 \\
15 \\
15 \\
17\end{array}$ & $\begin{array}{r}19 \\
16 \\
13 \\
34 \\
156\end{array}$ & $\begin{array}{l}15 \\
12 \\
12 \\
12 \\
12\end{array}$ \\
\hline $\begin{array}{l}11 \\
12 \\
13 \\
14 \\
15\end{array}$ & $\begin{array}{l}25 \\
24 \\
22 \\
22 \\
21\end{array}$ & $\begin{array}{l}13 \\
12 \\
13 \\
15 \\
17\end{array}$ & $\begin{array}{l}18 \\
17 \\
17 \\
17 \\
17\end{array}$ & $\begin{array}{l}13 \\
13 \\
12 \\
12 \\
12\end{array}$ & $\begin{array}{l}12 \\
12 \\
12 \\
12 \\
11\end{array}$ & $\begin{array}{l}22 \\
22 \\
22 \\
23 \\
24\end{array}$ & $\begin{array}{r}53 \\
191 \\
157 \\
94 \\
67\end{array}$ & $\begin{array}{l}31 \\
29 \\
28 \\
31 \\
30\end{array}$ & $\begin{array}{l}30 \\
24 \\
21 \\
19 \\
18\end{array}$ & $\begin{array}{l}22 \\
18 \\
27 \\
22 \\
17\end{array}$ & $\begin{array}{l}98 \\
45 \\
31 \\
25 \\
20\end{array}$ & $\begin{array}{l}12 \\
13 \\
13 \\
12 \\
11\end{array}$ \\
\hline $\begin{array}{l}16 \\
17 \\
18 \\
19 \\
20\end{array}$ & $\begin{array}{l}22 \\
23 \\
22 \\
21 \\
21\end{array}$ & $\begin{array}{l}17 \\
25 \\
45 \\
40 \\
34\end{array}$ & $\begin{array}{l}16 \\
16 \\
16 \\
16 \\
16\end{array}$ & $\begin{array}{l}12 \\
12 \\
13 \\
14 \\
14\end{array}$ & $\begin{array}{l}11 \\
11 \\
12 \\
12 \\
13\end{array}$ & $\begin{array}{r}23 \\
23 \\
29 \\
72 \\
200\end{array}$ & $\begin{array}{l}55 \\
48 \\
44 \\
40 \\
38\end{array}$ & $\begin{array}{l}26 \\
27 \\
29 \\
35 \\
35\end{array}$ & $\begin{array}{l}17 \\
17 \\
16 \\
16 \\
25\end{array}$ & $\begin{array}{l}14 \\
13 \\
12 \\
12 \\
12\end{array}$ & $\begin{array}{l}17 \\
17 \\
18 \\
18 \\
39\end{array}$ & $\begin{array}{c}11 \\
11 \\
11 \\
9.6 \\
10\end{array}$ \\
\hline $\begin{array}{l}21 \\
22 \\
23 \\
24 \\
25\end{array}$ & $\begin{array}{l}20 \\
17 \\
13 \\
15 \\
16\end{array}$ & $\begin{array}{l}31 \\
30 \\
35 \\
39 \\
35\end{array}$ & $\begin{array}{l}16 \\
15 \\
15 \\
15 \\
15\end{array}$ & $\begin{array}{l}14 \\
14 \\
13 \\
13 \\
13\end{array}$ & $\begin{array}{l}13 \\
13 \\
14 \\
14 \\
14\end{array}$ & $\begin{array}{l}270 \\
286 \\
350 \\
326 \\
146\end{array}$ & $\begin{array}{l}37 \\
35 \\
34 \\
33 \\
48\end{array}$ & $\begin{array}{l}34 \\
29 \\
28 \\
28 \\
25\end{array}$ & $\begin{array}{l}24 \\
20 \\
17 \\
14 \\
14\end{array}$ & $\begin{array}{l}12 \\
11 \\
12 \\
12 \\
22\end{array}$ & $\begin{array}{l}49 \\
35 \\
50 \\
47 \\
34\end{array}$ & $\begin{array}{l}11 \\
10 \\
10 \\
11 \\
11\end{array}$ \\
\hline $\begin{array}{l}26 \\
27 \\
28 \\
29 \\
30 \\
31\end{array}$ & $\begin{array}{c}15 \\
14 \\
12 \\
11 \\
9.6 \\
10\end{array}$ & $\begin{array}{r}33 \\
30 \\
28 \\
26 \\
25 \\
--\end{array}$ & $\begin{array}{l}14 \\
14 \\
14 \\
14 \\
14 \\
14\end{array}$ & $\begin{array}{l}13 \\
13 \\
12 \\
12 \\
11 \\
11\end{array}$ & $\begin{array}{r}15 \\
16 \\
18 \\
\hdashline- \\
\hdashline- \\
\hdashline-\end{array}$ & $\begin{array}{r}90 \\
80 \\
76 \\
84 \\
376 \\
379\end{array}$ & $\begin{array}{r}104 \\
91 \\
58 \\
47 \\
65 \\
---\end{array}$ & $\begin{array}{l}23 \\
21 \\
21 \\
21 \\
21 \\
29\end{array}$ & $\begin{array}{r}13 \\
13 \\
13 \\
41 \\
47 \\
-.-\end{array}$ & $\begin{array}{l}19 \\
16 \\
15 \\
13 \\
16 \\
20\end{array}$ & $\begin{array}{l}26 \\
25 \\
24 \\
24 \\
23 \\
19\end{array}$ & $\begin{array}{l}11 \\
10 \\
10 \\
10 \\
11\end{array}$ \\
\hline $\begin{array}{l}\text { TOTAL } \\
\text { MEAN } \\
\text { MAX } \\
\text { MIN } \\
\text { CFSM } \\
\text { IN. }\end{array}$ & $\begin{array}{r}682.6 \\
22.0 \\
37 \\
9.6 \\
.59 \\
.68\end{array}$ & $\begin{array}{r}658 \\
21.9 \\
45 \\
11 \\
.59 \\
.66\end{array}$ & $\begin{array}{r}533 \\
17.2 \\
24 \\
14 \\
.46 \\
.53\end{array}$ & $\begin{array}{r}399 \\
12.9 \\
14 \\
11 \\
.35 \\
.40\end{array}$ & $\begin{array}{r}348 \\
12.4 \\
18 \\
11 \\
.33 \\
.35\end{array}$ & $\begin{array}{r}3146 \\
101 \\
379 \\
19 \\
2.72 \\
3.15\end{array}$ & $\begin{array}{r}2242 \\
74.7 \\
191 \\
33 \\
2.01 \\
2.24\end{array}$ & $\begin{array}{r}1140 \\
36.8 \\
99 \\
21 \\
.99 \\
1.14\end{array}$ & $\begin{array}{r}658 \\
21.9 \\
47 \\
13 \\
.59 \\
.66\end{array}$ & $\begin{array}{r}578 \\
18.6 \\
45 \\
11 \\
.50 \\
.58\end{array}$ & $\begin{array}{r}1002 \\
32.3 \\
156 \\
13 \\
.87 \\
1.00\end{array}$ & $\begin{array}{r}360.6 \\
12.0 \\
18 \\
9.6 \\
.32 \\
.36\end{array}$ \\
\hline WTR YR & 179 TOTAL & 1174 & MEA & 32.2 & $\operatorname{MAX} 379$ & MIN 9.6 & CF & .87 & N 11.75 & & & \\
\hline
\end{tabular}

DISCHARGE, IN CUBIC FEET PER SECOND, WATER YEAR OCTOBER 1979 TO SEPTEMBER 1980

\begin{tabular}{|c|c|c|c|c|c|c|c|c|c|c|c|c|}
\hline DAY & ОСт & Nov & DEC & JAN & FEB & MAR & APR & MAY & JUN & JUL & AUG & SEP \\
\hline $\begin{array}{l}1 \\
2 \\
3 \\
4 \\
5\end{array}$ & $\begin{array}{l}10 \\
12 \\
11 \\
12 \\
12\end{array}$ & $\begin{array}{l}23 \\
21 \\
19 \\
16 \\
17\end{array}$ & $\begin{array}{l}22 \\
18 \\
17 \\
18 \\
19\end{array}$ & $\begin{array}{l}21 \\
20 \\
18 \\
17 \\
16\end{array}$ & $\begin{array}{l}11 \\
12 \\
12 \\
13 \\
14\end{array}$ & $\begin{array}{l}12 \\
12 \\
12 \\
12 \\
11\end{array}$ & $\begin{array}{l}32 \\
32 \\
37 \\
57 \\
63\end{array}$ & $\begin{array}{l}41 \\
33 \\
27 \\
24 \\
21\end{array}$ & $\begin{array}{l}39 \\
33 \\
33 \\
30 \\
42\end{array}$ & $\begin{array}{c}10 \\
10 \\
8.9 \\
8.6 \\
11\end{array}$ & $\begin{array}{l}10 \\
11 \\
12 \\
11 \\
13\end{array}$ & $\begin{array}{l}25 \\
28 \\
20 \\
18 \\
16\end{array}$ \\
\hline $\begin{array}{r}6 \\
7 \\
8 \\
9 \\
10\end{array}$ & $\begin{array}{l}13 \\
15 \\
17 \\
16 \\
14\end{array}$ & $\begin{array}{l}23 \\
22 \\
22 \\
19 \\
19\end{array}$ & $\begin{array}{l}21 \\
21 \\
20 \\
24 \\
26\end{array}$ & $\begin{array}{l}15 \\
15 \\
14 \\
13 \\
13\end{array}$ & $\begin{array}{l}14 \\
15 \\
15 \\
14 \\
14\end{array}$ & $\begin{array}{l}11 \\
10 \\
10 \\
11 \\
13\end{array}$ & $\begin{array}{r}53 \\
64 \\
181 \\
213 \\
145\end{array}$ & $\begin{array}{l}19 \\
15 \\
17 \\
15 \\
14\end{array}$ & $\begin{array}{r}98 \\
139 \\
196 \\
117 \\
38\end{array}$ & $\begin{array}{c}10 \\
9.7 \\
9.6 \\
9.6 \\
8.6\end{array}$ & $\begin{array}{l}12 \\
17 \\
86 \\
67 \\
45\end{array}$ & $\begin{array}{l}15 \\
15 \\
14 \\
17 \\
16\end{array}$ \\
\hline $\begin{array}{l}11 \\
12 \\
13 \\
14 \\
15\end{array}$ & $\begin{array}{l}15 \\
17 \\
13 \\
12 \\
13\end{array}$ & $\begin{array}{l}28 \\
17 \\
18 \\
18 \\
18\end{array}$ & $\begin{array}{l}28 \\
25 \\
20 \\
14 \\
14\end{array}$ & $\begin{array}{l}13 \\
12 \\
12 \\
11 \\
11\end{array}$ & $\begin{array}{l}14 \\
15 \\
15 \\
15 \\
15\end{array}$ & $\begin{array}{l}14 \\
13 \\
13 \\
13 \\
14\end{array}$ & $\begin{array}{l}85 \\
70 \\
61 \\
55 \\
59\end{array}$ & $\begin{array}{l}18 \\
14 \\
23 \\
36 \\
39\end{array}$ & $\begin{array}{l}25 \\
22 \\
20 \\
24 \\
27\end{array}$ & $\begin{array}{c}8.1 \\
8.1 \\
8.3 \\
8.3 \\
10\end{array}$ & $\begin{array}{l}29 \\
25 \\
21 \\
20 \\
17\end{array}$ & $\begin{array}{l}14 \\
19 \\
30 \\
32 \\
23\end{array}$ \\
\hline $\begin{array}{l}16 \\
17 \\
18 \\
19 \\
20\end{array}$ & $\begin{array}{l}13 \\
14 \\
14 \\
26 \\
18\end{array}$ & $\begin{array}{l}19 \\
19 \\
18 \\
18 \\
18\end{array}$ & $\begin{array}{l}13 \\
13 \\
13 \\
14 \\
15\end{array}$ & $\begin{array}{l}15 \\
68 \\
60 \\
26 \\
19\end{array}$ & $\begin{array}{l}14 \\
14 \\
13 \\
14 \\
16\end{array}$ & $\begin{array}{l}15 \\
20 \\
45 \\
62 \\
56\end{array}$ & $\begin{array}{l}78 \\
66 \\
56 \\
52 \\
47\end{array}$ & $\begin{array}{l}36 \\
31 \\
46 \\
52 \\
38\end{array}$ & $\begin{array}{l}20 \\
18 \\
16 \\
28 \\
34\end{array}$ & $\begin{array}{c}15 \\
13 \\
10 \\
9.9 \\
73\end{array}$ & $\begin{array}{l}14 \\
15 \\
16 \\
15 \\
56\end{array}$ & $\begin{array}{l}28 \\
34 \\
25 \\
20 \\
36\end{array}$ \\
\hline $\begin{array}{l}21 \\
22 \\
23 \\
24 \\
25\end{array}$ & $\begin{array}{l}19 \\
22 \\
32 \\
37 \\
30\end{array}$ & $\begin{array}{l}24 \\
35 \\
34 \\
27 \\
24\end{array}$ & $\begin{array}{l}16 \\
18 \\
25 \\
54 \\
91\end{array}$ & $\begin{array}{l}15 \\
13 \\
12 \\
11 \\
10\end{array}$ & $\begin{array}{l}17 \\
21 \\
19 \\
17 \\
15\end{array}$ & $\begin{array}{l}43 \\
33 \\
25 \\
24 \\
32\end{array}$ & $\begin{array}{l}43 \\
40 \\
34 \\
31 \\
29\end{array}$ & $\begin{array}{l}29 \\
26 \\
22 \\
20 \\
20\end{array}$ & $\begin{array}{l}24 \\
19 \\
17 \\
15 \\
14\end{array}$ & $\begin{array}{l}69 \\
56 \\
22 \\
17 \\
15\end{array}$ & $\begin{array}{l}67 \\
46 \\
26 \\
22 \\
22\end{array}$ & $\begin{array}{r}34 \\
201 \\
126 \\
64 \\
46\end{array}$ \\
\hline $\begin{array}{l}26 \\
27 \\
28 \\
29 \\
30 \\
31\end{array}$ & $\begin{array}{l}22 \\
22 \\
21 \\
19 \\
19 \\
18\end{array}$ & $\begin{array}{l}38 \\
52 \\
44 \\
38 \\
23 \\
--\end{array}$ & $\begin{array}{l}66 \\
39 \\
29 \\
26 \\
25 \\
22\end{array}$ & $\begin{array}{r}9.8 \\
9.4 \\
9.1 \\
9.0 \\
9.0 \\
10\end{array}$ & $\begin{array}{r}14 \\
14 \\
13 \\
22 \\
--- \\
--\end{array}$ & $\begin{array}{l}39 \\
38 \\
35 \\
33 \\
32 \\
32\end{array}$ & $\begin{array}{l}29 \\
27 \\
27 \\
35 \\
47 \\
--\end{array}$ & $\begin{array}{l}18 \\
17 \\
18 \\
40 \\
45 \\
47\end{array}$ & $\begin{array}{c}13 \\
11 \\
13 \\
13 \\
9.8 \\
---\end{array}$ & $\begin{array}{c}14 \\
14 \\
12 \\
11 \\
9.9 \\
12\end{array}$ & $\begin{array}{l}32 \\
29 \\
36 \\
40 \\
32 \\
25\end{array}$ & $\begin{array}{r}40 \\
34 \\
29 \\
27 \\
26 \\
--\end{array}$ \\
\hline $\begin{array}{l}\text { TOTAL } \\
\text { MEAN } \\
\text { MAX } \\
\text { MIN } \\
\text { CFSM } \\
\text { IN. }\end{array}$ & $\begin{array}{r}548 \\
17.7 \\
37 \\
10 \\
.48 \\
.55\end{array}$ & $\begin{array}{r}731 \\
24.4 \\
52 \\
16 \\
.66 \\
.73\end{array}$ & $\begin{array}{r}786 \\
25.4 \\
91 \\
13 \\
.68 \\
.79\end{array}$ & $\begin{array}{r}526.3 \\
17.0 \\
68 \\
9.0 \\
.46 \\
.53\end{array}$ & $\begin{array}{r}431 \\
14.9 \\
22 \\
11 \\
.40 \\
.43\end{array}$ & $\begin{array}{r}745 \\
24.0 \\
62 \\
10 \\
.65 \\
.74\end{array}$ & $\begin{array}{r}1848 \\
61.6 \\
213 \\
27 \\
1.66 \\
1.85\end{array}$ & $\begin{array}{r}861 \\
27.8 \\
52 \\
14 \\
.75 \\
.86\end{array}$ & $\begin{array}{r}1147.8 \\
38.3 \\
196 \\
9.8 \\
1.03 \\
1.15\end{array}$ & $\begin{array}{r}511.6 \\
16.5 \\
73 \\
8.1 \\
.44 \\
.51\end{array}$ & $\begin{array}{r}889 \\
28.7 \\
86 \\
10 \\
.77 \\
.89\end{array}$ & $\begin{array}{r}1072 \\
35.7 \\
201 \\
14 \\
.96 \\
1.07\end{array}$ \\
\hline
\end{tabular}

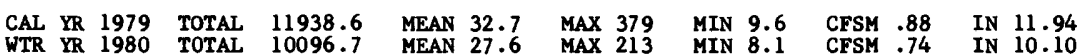


Table 11. Stream discharges for Onion River near Sheboygan Falls, 1979 and 1980 water years

\begin{tabular}{|c|c|c|c|c|c|c|c|c|c|c|c|c|}
\hline \multirow[b]{2}{*}{ DAY } & \multicolumn{12}{|c|}{ OCTOBER 1978} \\
\hline & OCT & Nov & DEC & JAN & FEB & MAR & APR & MAY & JUN & JUL & AUG & SEP \\
\hline $\begin{array}{l}1 \\
2 \\
3 \\
4 \\
5\end{array}$ & $\begin{array}{l}90 \\
76 \\
58 \\
48 \\
40\end{array}$ & $\begin{array}{l}18 \\
19 \\
19 \\
19 \\
19\end{array}$ & $\begin{array}{l}40 \\
39 \\
38 \\
36 \\
36\end{array}$ & $\begin{array}{l}22 \\
22 \\
22 \\
21 \\
21\end{array}$ & $\begin{array}{l}17 \\
17 \\
17 \\
17 \\
17\end{array}$ & $\begin{array}{l}33 \\
34 \\
37 \\
45 \\
45\end{array}$ & $\begin{array}{r}1180 \\
628 \\
396 \\
387 \\
430\end{array}$ & $\begin{array}{l}210 \\
180 \\
240 \\
170 \\
140\end{array}$ & $\begin{array}{l}34 \\
35 \\
30 \\
45 \\
36\end{array}$ & $\begin{array}{l}75 \\
66 \\
51 \\
36 \\
34\end{array}$ & $\begin{array}{l}26 \\
22 \\
20 \\
18 \\
18\end{array}$ & $\begin{array}{l}48 \\
45 \\
40 \\
33 \\
30\end{array}$ \\
\hline $\begin{array}{r}6 \\
7 \\
8 \\
9 \\
10\end{array}$ & $\begin{array}{r}100 \\
96 \\
82 \\
72 \\
62\end{array}$ & $\begin{array}{l}19 \\
19 \\
19 \\
19 \\
19\end{array}$ & $\begin{array}{l}35 \\
34 \\
33 \\
33 \\
33\end{array}$ & $\begin{array}{l}21 \\
20 \\
20 \\
20 \\
20\end{array}$ & $\begin{array}{l}17 \\
18 \\
18 \\
19 \\
20\end{array}$ & $\begin{array}{l}44 \\
44 \\
44 \\
44 \\
44\end{array}$ & $\begin{array}{l}336 \\
265 \\
185 \\
156 \\
153\end{array}$ & $\begin{array}{r}120 \\
100 \\
84 \\
74 \\
66\end{array}$ & $\begin{array}{r}33 \\
70 \\
58 \\
48 \\
110\end{array}$ & $\begin{array}{l}31 \\
25 \\
22 \\
20 \\
18\end{array}$ & $\begin{array}{r}28 \\
25 \\
20 \\
20 \\
200\end{array}$ & $\begin{array}{l}29 \\
26 \\
21 \\
19 \\
18\end{array}$ \\
\hline $\begin{array}{l}11 \\
12 \\
13 \\
14 \\
15\end{array}$ & $\begin{array}{l}54 \\
49 \\
42 \\
37 \\
33\end{array}$ & $\begin{array}{l}19 \\
19 \\
22 \\
27 \\
35\end{array}$ & $\begin{array}{l}33 \\
32 \\
31 \\
30 \\
29\end{array}$ & $\begin{array}{l}20 \\
20 \\
20 \\
15 \\
13\end{array}$ & $\begin{array}{l}20 \\
20 \\
20 \\
20 \\
20\end{array}$ & $\begin{array}{l}44 \\
46 \\
49 \\
50 \\
50\end{array}$ & $\begin{array}{l}164 \\
575 \\
698 \\
517 \\
305\end{array}$ & $\begin{array}{l}60 \\
58 \\
54 \\
52 \\
56\end{array}$ & $\begin{array}{l}80 \\
60 \\
50 \\
42 \\
38\end{array}$ & $\begin{array}{l}21 \\
25 \\
22 \\
29 \\
25\end{array}$ & $\begin{array}{r}302 \\
186 \\
88 \\
61 \\
45\end{array}$ & $\begin{array}{l}18 \\
19 \\
19 \\
18 \\
17\end{array}$ \\
\hline $\begin{array}{l}16 \\
17 \\
18 \\
19 \\
20\end{array}$ & $\begin{array}{l}35 \\
35 \\
34 \\
32 \\
33\end{array}$ & $\begin{array}{r}30 \\
50 \\
100 \\
90 \\
70\end{array}$ & $\begin{array}{l}29 \\
28 \\
27 \\
27 \\
27\end{array}$ & $\begin{array}{l}12 \\
13 \\
15 \\
17 \\
18\end{array}$ & $\begin{array}{l}19 \\
19 \\
20 \\
20 \\
20\end{array}$ & $\begin{array}{r}50 \\
50 \\
80 \\
270 \\
360\end{array}$ & $\begin{array}{r}195 \\
153 \\
126 \\
109 \\
96\end{array}$ & $\begin{array}{l}48 \\
52 \\
58 \\
90 \\
86\end{array}$ & $\begin{array}{l}36 \\
33 \\
31 \\
30 \\
60\end{array}$ & $\begin{array}{l}19 \\
16 \\
14 \\
15 \\
15\end{array}$ & $\begin{array}{l}35 \\
31 \\
30 \\
30 \\
44\end{array}$ & $\begin{array}{l}16 \\
16 \\
15 \\
14 \\
13\end{array}$ \\
\hline $\begin{array}{l}21 \\
22 \\
23 \\
24 \\
25\end{array}$ & $\begin{array}{l}32 \\
27 \\
20 \\
32 \\
36\end{array}$ & $\begin{array}{r}56 \\
46 \\
60 \\
100 \\
84\end{array}$ & $\begin{array}{l}26 \\
26 \\
26 \\
25 \\
25\end{array}$ & $\begin{array}{l}19 \\
19 \\
19 \\
19 \\
19\end{array}$ & $\begin{array}{l}21 \\
22 \\
24 \\
26 \\
28\end{array}$ & $\begin{array}{l}1270 \\
1460 \\
1840 \\
1780 \\
774\end{array}$ & $\begin{array}{l}88 \\
82 \\
75 \\
70 \\
85\end{array}$ & $\begin{array}{l}70 \\
60 \\
52 \\
48 \\
44\end{array}$ & $\begin{array}{l}50 \\
34 \\
27 \\
22 \\
20\end{array}$ & $\begin{array}{l}16 \\
17 \\
15 \\
14 \\
16\end{array}$ & $\begin{array}{r}128 \\
98 \\
91 \\
187 \\
158\end{array}$ & $\begin{array}{l}14 \\
14 \\
14 \\
14 \\
14\end{array}$ \\
\hline $\begin{array}{l}26 \\
27 \\
28 \\
29 \\
30 \\
31\end{array}$ & $\begin{array}{l}35 \\
27 \\
22 \\
17 \\
17 \\
18\end{array}$ & $\begin{array}{r}70 \\
58 \\
52 \\
47 \\
44 \\
---\end{array}$ & $\begin{array}{l}24 \\
24 \\
24 \\
23 \\
23 \\
23\end{array}$ & $\begin{array}{l}18 \\
18 \\
18 \\
18 \\
18 \\
18\end{array}$ & $\begin{array}{r}29 \\
30 \\
32 \\
--- \\
---\end{array}$ & $\begin{array}{r}500 \\
400 \\
370 \\
354 \\
956 \\
1770\end{array}$ & $\begin{array}{l}235 \\
284 \\
188 \\
131 \\
218 \\
---\end{array}$ & $\begin{array}{l}40 \\
38 \\
37 \\
36 \\
48 \\
52\end{array}$ & $\begin{array}{l}18 \\
17 \\
17 \\
46 \\
98 \\
-\cdots\end{array}$ & $\begin{array}{l}26 \\
24 \\
20 \\
18 \\
17 \\
23\end{array}$ & $\begin{array}{l}89 \\
71 \\
68 \\
60 \\
54 \\
47\end{array}$ & $\begin{array}{r}14 \\
15 \\
14 \\
13 \\
14 \\
--\end{array}$ \\
\hline $\begin{array}{l}\text { TOTAL } \\
\text { MEAN } \\
\text { MAX } \\
\text { MIN } \\
\text { CFSM } \\
\text { IN. }\end{array}$ & $\begin{array}{r}1391 \\
44.9 \\
100 \\
17 \\
.48 \\
.55\end{array}$ & $\begin{array}{r}1268 \\
42.3 \\
100 \\
18 \\
.45 \\
.50\end{array}$ & $\begin{array}{r}919 \\
29.6 \\
40 \\
23 \\
.32 \\
.36\end{array}$ & $\begin{array}{r}575 \\
18.5 \\
22 \\
12 \\
.20 \\
.23\end{array}$ & $\begin{array}{r}587 \\
21.0 \\
32 \\
17 \\
.22 \\
.23\end{array}$ & $\begin{array}{r}12937 \\
417 \\
1840 \\
33 \\
4.43 \\
5.11\end{array}$ & $\begin{array}{r}8510 \\
284 \\
1180 \\
70 \\
3.02 \\
3.36\end{array}$ & $\begin{array}{r}2523 \\
81.4 \\
240 \\
36 \\
.87 \\
1.00\end{array}$ & $\begin{array}{r}1308 \\
43.6 \\
110 \\
17 \\
.46 \\
.52\end{array}$ & $\begin{array}{r}785 \\
25.3 \\
75 \\
14 \\
.27 \\
.31\end{array}$ & $\begin{array}{r}2300 \\
74.2 \\
302 \\
18 \\
.79 \\
.91\end{array}$ & $\begin{array}{r}614 \\
20.5 \\
48 \\
13 \\
.22 \\
.24\end{array}$ \\
\hline WTR Y & 9 тот & 33717 & MEAN & & 1840 & MIN 1 & CFS & 98 & 13.33 & & & \\
\hline
\end{tabular}

DISCHARGE, IN CUBIC FEET PER SECOND, WATER YEAR OCTOBER 1979 TO SEPTEMBER 1980

\begin{tabular}{|c|c|c|c|c|c|c|c|c|c|c|c|c|}
\hline DAY & OCT & NOV & DEC & JAN & FEB & MAR & APR & MAY & JUN & JUL & AUG & SEP \\
\hline $\begin{array}{l}1 \\
2 \\
3 \\
4 \\
5\end{array}$ & $\begin{array}{l}15 \\
15 \\
16 \\
15 \\
16\end{array}$ & $\begin{array}{l}22 \\
24 \\
22 \\
21 \\
19\end{array}$ & $\begin{array}{l}24 \\
22 \\
21 \\
21 \\
21\end{array}$ & $\begin{array}{l}31 \\
28 \\
27 \\
25 \\
24\end{array}$ & $\begin{array}{l}32 \\
34 \\
35 \\
36 \\
36\end{array}$ & $\begin{array}{l}31 \\
27 \\
24 \\
22 \\
25\end{array}$ & $\begin{array}{r}50 \\
49 \\
53 \\
146 \\
247\end{array}$ & $\begin{array}{l}58 \\
52 \\
45 \\
39 \\
35\end{array}$ & $\begin{array}{l}38 \\
34 \\
32 \\
31 \\
36\end{array}$ & $\begin{array}{l}16 \\
16 \\
16 \\
16 \\
15\end{array}$ & $\begin{array}{l}17 \\
16 \\
17 \\
18 \\
18\end{array}$ & $\begin{array}{l}48 \\
46 \\
43 \\
33 \\
31\end{array}$ \\
\hline $\begin{array}{r}6 \\
7 \\
8 \\
9 \\
10\end{array}$ & $\begin{array}{l}17 \\
17 \\
19 \\
21 \\
20\end{array}$ & $\begin{array}{l}20 \\
25 \\
25 \\
23 \\
21\end{array}$ & $\begin{array}{l}21 \\
22 \\
24 \\
35 \\
25\end{array}$ & $\begin{array}{l}23 \\
22 \\
21 \\
20 \\
19\end{array}$ & $\begin{array}{l}35 \\
34 \\
32 \\
28 \\
26\end{array}$ & $\begin{array}{l}26 \\
18 \\
18 \\
19 \\
19\end{array}$ & $\begin{array}{l}155 \\
137 \\
267 \\
428 \\
411\end{array}$ & $\begin{array}{l}33 \\
30 \\
27 \\
28 \\
26\end{array}$ & $\begin{array}{l}137 \\
179 \\
199 \\
193 \\
144\end{array}$ & $\begin{array}{l}17 \\
17 \\
16 \\
14 \\
14\end{array}$ & $\begin{array}{r}20 \\
20 \\
130 \\
306 \\
188\end{array}$ & $\begin{array}{l}27 \\
25 \\
24 \\
25 \\
28\end{array}$ \\
\hline $\begin{array}{l}11 \\
12 \\
13 \\
14 \\
15\end{array}$ & $\begin{array}{l}19 \\
18 \\
20 \\
17 \\
16\end{array}$ & $\begin{array}{l}20 \\
21 \\
20 \\
20 \\
20\end{array}$ & $\begin{array}{l}22 \\
20 \\
18 \\
17 \\
16\end{array}$ & $\begin{array}{l}18 \\
18 \\
17 \\
17 \\
30\end{array}$ & $\begin{array}{l}25 \\
25 \\
25 \\
24 \\
23\end{array}$ & $\begin{array}{l}18 \\
16 \\
17 \\
18 \\
20\end{array}$ & $\begin{array}{l}272 \\
171 \\
134 \\
109 \\
111\end{array}$ & $\begin{array}{l}27 \\
27 \\
28 \\
40 \\
48\end{array}$ & $\begin{array}{l}65 \\
49 \\
41 \\
39 \\
44\end{array}$ & $\begin{array}{l}14 \\
13 \\
13 \\
12 \\
14\end{array}$ & $\begin{array}{r}111 \\
90 \\
75 \\
58 \\
46\end{array}$ & $\begin{array}{l}26 \\
25 \\
36 \\
43 \\
41\end{array}$ \\
\hline $\begin{array}{l}16 \\
17 \\
18 \\
19 \\
20\end{array}$ & $\begin{array}{l}18 \\
18 \\
18 \\
19 \\
30\end{array}$ & $\begin{array}{l}21 \\
22 \\
21 \\
21 \\
21\end{array}$ & $\begin{array}{l}15 \\
15 \\
15 \\
15 \\
16\end{array}$ & $\begin{array}{r}60 \\
170 \\
160 \\
150 \\
100\end{array}$ & $\begin{array}{l}22 \\
20 \\
19 \\
20 \\
23\end{array}$ & $\begin{array}{r}25 \\
35 \\
50 \\
210 \\
200\end{array}$ & $\begin{array}{r}193 \\
159 \\
125 \\
104 \\
90\end{array}$ & $\begin{array}{l}47 \\
42 \\
46 \\
59 \\
56\end{array}$ & $\begin{array}{l}43 \\
33 \\
29 \\
31 \\
47\end{array}$ & $\begin{array}{r}22 \\
29 \\
22 \\
17 \\
111\end{array}$ & $\begin{array}{l}38 \\
34 \\
35 \\
35 \\
85\end{array}$ & $\begin{array}{l}36 \\
81 \\
65 \\
47 \\
64\end{array}$ \\
\hline $\begin{array}{l}21 \\
22 \\
23 \\
24 \\
25\end{array}$ & $\begin{array}{l}21 \\
20 \\
25 \\
33 \\
37\end{array}$ & $\begin{array}{l}22 \\
32 \\
40 \\
34 \\
28\end{array}$ & $\begin{array}{r}16 \\
17 \\
20 \\
25 \\
150\end{array}$ & $\begin{array}{l}80 \\
66 \\
56 \\
50 \\
45\end{array}$ & $\begin{array}{l}30 \\
40 \\
60 \\
56 \\
52\end{array}$ & $\begin{array}{r}100 \\
70 \\
52 \\
43 \\
45\end{array}$ & $\begin{array}{l}78 \\
70 \\
62 \\
54 \\
50\end{array}$ & $\begin{array}{l}44 \\
37 \\
33 \\
31 \\
29\end{array}$ & $\begin{array}{l}44 \\
33 \\
29 \\
24 \\
22\end{array}$ & $\begin{array}{r}212 \\
110 \\
57 \\
36 \\
28\end{array}$ & $\begin{array}{r}137 \\
100 \\
63 \\
44 \\
38\end{array}$ & $\begin{array}{l}116 \\
464 \\
675 \\
686 \\
427\end{array}$ \\
\hline $\begin{array}{l}26 \\
27 \\
28 \\
29 \\
30 \\
31\end{array}$ & $\begin{array}{l}30 \\
24 \\
24 \\
24 \\
21 \\
21\end{array}$ & $\begin{array}{l}34 \\
60 \\
58 \\
40 \\
26 \\
---\end{array}$ & $\begin{array}{r}130 \\
60 \\
45 \\
40 \\
35 \\
33\end{array}$ & $\begin{array}{l}42 \\
38 \\
36 \\
33 \\
31 \\
31\end{array}$ & $\begin{array}{r}54 \\
50 \\
37 \\
86 \\
--- \\
---\end{array}$ & $\begin{array}{l}56 \\
82 \\
86 \\
71 \\
76 \\
59\end{array}$ & $\begin{array}{r}47 \\
44 \\
43 \\
46 \\
54 \\
--.\end{array}$ & $\begin{array}{l}27 \\
25 \\
24 \\
26 \\
39 \\
41\end{array}$ & $\begin{array}{r}21 \\
19 \\
19 \\
21 \\
20 \\
-\end{array}$ & $\begin{array}{l}25 \\
26 \\
23 \\
20 \\
17 \\
16\end{array}$ & $\begin{array}{l}46 \\
50 \\
50 \\
73 \\
64 \\
51\end{array}$ & $\begin{array}{r}227 \\
145 \\
111 \\
92 \\
77 \\
---\end{array}$ \\
\hline $\begin{array}{l}\text { TOTAL } \\
\text { MEAN } \\
\text { MAX } \\
\text { MIN } \\
\text { CFSM } \\
\text { IN. }\end{array}$ & $\begin{array}{r}644 \\
20.8 \\
37 \\
15 \\
.22 \\
.25\end{array}$ & $\begin{array}{r}803 \\
26.8 \\
60 \\
19 \\
.29 \\
.32\end{array}$ & $\begin{array}{r}976 \\
31.5 \\
150 \\
15 \\
.34 \\
.39\end{array}$ & $\begin{array}{r}1488 \\
48.0 \\
170 \\
17 \\
.51 \\
.59\end{array}$ & $\begin{array}{r}1019 \\
35.1 \\
86 \\
19 \\
.37 \\
.40\end{array}$ & $\begin{array}{r}1578 \\
50.9 \\
210 \\
16 \\
.54 \\
.62\end{array}$ & $\begin{array}{r}3959 \\
132 \\
428 \\
43 \\
1.40 \\
1.57\end{array}$ & $\begin{array}{r}1149 \\
37.1 \\
59 \\
24 \\
.39 \\
.45\end{array}$ & $\begin{array}{r}1696 \\
56.5 \\
199 \\
19 \\
.60 \\
.67\end{array}$ & $\begin{array}{r}994 \\
32.1 \\
212 \\
12 \\
.34 \\
.39\end{array}$ & $\begin{array}{r}2073 \\
66.9 \\
306 \\
16 \\
.71 \\
.82\end{array}$ & $\begin{array}{r}3814 \\
127 \\
686 \\
24 \\
1.35 \\
1.51\end{array}$ \\
\hline
\end{tabular}

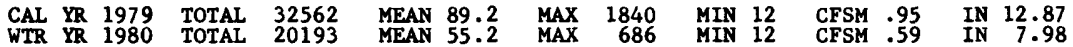


Table 12. Water and bed-material analyses from Onion River at Hingham, 1979 and 1980 water years

\begin{tabular}{|c|c|c|c|c|c|c|c|c|}
\hline DATE & TIME & $\begin{array}{l}\text { STREAM- } \\
\text { FLOW, } \\
\text { INSTAN- } \\
\text { TANEOUS } \\
\text { (CFS) }\end{array}$ & $\begin{array}{c}\text { ALKA- } \\
\text { LINITY } \\
\text { FIELD } \\
\text { (MG/L } \\
\text { AS } \\
\text { CACO3) }\end{array}$ & $\begin{array}{l}\text { CHLO- } \\
\text { RIDE, } \\
\text { DIS- } \\
\text { SOLVED } \\
\text { (MG/L } \\
\text { AS CL) }\end{array}$ & $\begin{array}{l}\text { SOLIDS, } \\
\text { RESIDUE } \\
\text { AT } 180 \\
\text { DEG. C } \\
\text { DIS - } \\
\text { SOLVED } \\
\text { (MG/L) }\end{array}$ & $\begin{array}{c}\text { SOLIDS, } \\
\text { DIS- } \\
\text { SOLVED } \\
\text { (TONS } \\
\text { PER } \\
\text { AC-FT) }\end{array}$ & $\begin{array}{c}\text { SOLIDS, } \\
\text { DIS- } \\
\text { SOLVED } \\
\text { (TONS } \\
\text { PER } \\
\text { DAY) }\end{array}$ & $\begin{array}{c}\text { NITRO- } \\
\text { GEN, } \\
\text { NITRATE } \\
\text { TOTAL } \\
\text { (MG/L } \\
\text { AS N) }\end{array}$ \\
\hline $\begin{array}{c}\text { DEC }, \\
06 . .\end{array}$ & ${ }^{1978} 1525$ & 20 & 320 & 24 & 408 & .55 & 22 & 1.90 \\
\hline $\begin{array}{c}\text { FEB } \\
28 . .\end{array}$ & ${ }^{1979} 1230$ & 18 & 320 & 20 & 399 & .54 & 19 & 1.18 \\
\hline $\begin{array}{r}\text { MAR } \\
20 \ldots \\
20 \ldots \\
23 \ldots \\
23 \ldots \\
23 \ldots \\
24 \ldots \\
30 \ldots \\
30 \ldots \\
30 \ldots \\
30 \ldots \\
30 \ldots \\
31 \ldots\end{array}$ & $\begin{array}{l}1345 \\
1515 \\
0545 \\
0745 \\
1900 \\
0230 \\
0230 \\
0330 \\
1345 \\
1615 \\
2345 \\
0215\end{array}$ & $\begin{array}{l}200 \\
200 \\
322 \\
324 \\
387 \\
428 \\
162 \\
189 \\
428 \\
578 \\
526 \\
495\end{array}$ & $\begin{array}{r}-- \\
153 \\
144 \\
-- \\
126 \\
-- \\
-- \\
171 \\
-\overline{9} \\
99 \\
-\overline{99}\end{array}$ & $\begin{array}{ll}20 & - \\
17 & \\
17 & - \\
& -- \\
20 & - \\
13 & - \\
11 & -\end{array}$ & $\begin{array}{r}-\overline{247} \\
230 \\
-- \\
203 \\
-- \\
-\overline{-} \\
278 \\
\overline{--} \\
168 \\
\overline{159}\end{array}$ & $\begin{array}{l}-\overline{-} \\
.34 \\
.31 \\
-- \\
.28 \\
-- \\
-- \\
.38 \\
-\overline{23} \\
. \overline{22}\end{array}$ & $\begin{array}{l}133^{--} \\
200 \\
212^{--} \\
142^{--} \\
262^{--} \\
213^{--}\end{array}$ & $\begin{array}{c}2.87 \\
2.97 \\
3.07 \\
2.57 \\
2.27 \\
1.87 \\
--\end{array}$ \\
\hline $\begin{array}{c}\text { APR } \\
01 \ldots \\
01 \ldots\end{array}$ & $\begin{array}{l}0815 \\
1045\end{array}$ & $\begin{array}{l}182 \\
170\end{array}$ & $\begin{array}{r}126 \\
--\end{array}$ & 14 & $\begin{array}{r}193 \\
--\end{array}$ & .26 & 95 & $2.08^{--}$ \\
\hline $\begin{array}{l}\text { MAY } \\
25 \ldots\end{array}$ & 1015 & 25 & 290 & 20 & 395 & .54 & 27 & 1.27 \\
\hline $\begin{array}{r}\text { JUN } \\
28 \ldots . \\
29 \ldots \\
29 \ldots \\
29 \ldots \\
29 \ldots \\
29 \ldots \\
30 \ldots \\
30 \ldots \\
30 \ldots \\
30 \ldots\end{array}$ & $\begin{array}{l}0930 \\
0615 \\
0900 \\
1015 \\
1300 \\
2015 \\
0615 \\
0900 \\
1500 \\
1900\end{array}$ & $\begin{array}{l}13 \\
44 \\
52 \\
52 \\
52 \\
45 \\
49 \\
49 \\
45 \\
44\end{array}$ & $\begin{array}{r}280 \\
-\overline{1} \\
144 \\
-- \\
189 \\
-- \\
-- \\
171 \\
-- \\
--\end{array}$ & $\begin{array}{ll}18 & \\
16 & - \\
14 & -- \\
& - \\
23 & - \\
& - \\
& -\end{array}$ & $\begin{array}{r}365 \\
-\overline{-} \\
257 \\
-\overline{-} \\
293 \\
-- \\
-\overline{2} \\
312 \\
-- \\
--\end{array}$ & $\begin{array}{r}.50 \\
.35 \\
.35 \\
-\overline{.40} \\
-- \\
-\overline{-} \\
.42 \\
-- \\
--\end{array}$ & $\begin{array}{c}13 \\
36 \\
41^{--} \\
41^{--} \\
-- \\
--\end{array}$ & $\begin{array}{r}.540 \\
.780 \\
1.41 \\
1.11^{--} \\
1.61 \\
1.31 \\
1.32\end{array}$ \\
\hline $\begin{array}{l}\text { JULL } \\
01 \ldots \\
01 \ldots\end{array}$ & $\begin{array}{l}0915 \\
1300\end{array}$ & $\begin{array}{l}44 \\
46\end{array}$ & 194 & $15^{--}$ & $28 \overline{4}$ &.$\overline{-}$ & $35^{--}$ & $\begin{array}{r}1.33 \\
-.\end{array}$ \\
\hline $\begin{array}{l}\text { AUG } \\
08 \ldots\end{array}$ & 1445 & 13 & 230 & 16 & 301 & .41 & 11 & .160 \\
\hline
\end{tabular}


Table 12. Water and bed-material analyses from Onion River at Hingham, 1979 and 1980 water years-Continued

\begin{tabular}{|c|c|c|c|c|c|c|c|c|}
\hline DATE & $\begin{array}{l}\text { NITRO- } \\
\text { GEN } \\
\text { NITRITE } \\
\text { TOTAL } \\
\text { (MG/L } \\
\text { AS N) }\end{array}$ & $\begin{array}{l}\text { NITRO- } \\
\text { GEN } \\
\text { NO2+NỎ3 } \\
\text { TOTAL } \\
\text { (MG/L } \\
\text { AS N) }\end{array}$ & $\begin{array}{l}\text { NITRO- } \\
\text { GEN, } \\
\text { AMMONIA } \\
\text { TOTAL } \\
\text { (MG/L } \\
\text { AS N) }\end{array}$ & $\begin{array}{c}\text { NITRO- } \\
\text { GEN, } \\
\text { ORGANIC } \\
\text { TOTAL } \\
\text { (MG/L } \\
\text { AS N) }\end{array}$ & $\begin{array}{l}\text { NITRO- } \\
\text { GEN, AM- } \\
\text { MONIA + } \\
\text { ORGANIC } \\
\text { TOTAL } \\
\text { (MG/L } \\
\text { AS N) }\end{array}$ & $\begin{array}{l}\text { NITRO- } \\
\text { GEN, } \\
\text { TOTAL } \\
\text { (MG/L } \\
\text { AS N) }\end{array}$ & $\begin{array}{c}\text { PHOS- } \\
\text { PHORUS, } \\
\text { TOTAL } \\
\text { (MG/L } \\
\text { AS P) }\end{array}$ & $\begin{array}{c}\text { PHOS- } \\
\text { PHORUS, } \\
\text { ORTHO, } \\
\text { TOTAL } \\
\text { (MG/L } \\
\text { AS P) }\end{array}$ \\
\hline $\begin{array}{c}\text { DEC }, \\
06 . .\end{array}$ & 1978 & 1.90 & -- & -- & -- & - & .060 & -- \\
\hline $\begin{array}{c}\text { FEB } \\
28 . .\end{array}$ & $\begin{array}{ll}1979.020\end{array}$ & 1.20 & .120 & .35 & .47 & 1.7 & .090 & .070 \\
\hline $\begin{array}{r}\text { MAR } \\
20 \ldots \\
20 \ldots \\
23 \ldots \\
23 \ldots \\
23 \ldots \\
24 \ldots \\
30 \ldots \\
30 \ldots \\
30 \ldots \\
30 \ldots \\
30 \ldots \\
31 \ldots\end{array}$ & $\begin{array}{r}.030 \\
-- \\
-0 \\
.030 \\
-- \\
.030 \\
.030 \\
-- \\
.030 \\
- \\
.030 \\
--\end{array}$ & $\begin{array}{c}2.90 \\
-- \\
3.00^{--} \\
3.10^{--} \\
2.60 \\
2.30^{--} \\
1.90^{--} \\
\cdots\end{array}$ & $\begin{array}{r}.170 \\
-- \\
- \\
.100 \\
-- \\
.090 \\
.100 \\
-- \\
.150 \\
-- \\
.150 \\
--\end{array}$ & $\begin{array}{c}1.0 \\
=- \\
.80 \\
=- \\
1.0 \\
1.1 \\
-- \\
2.2 \\
1.3 \\
--\end{array}$ & $\begin{array}{c}1.1 \\
-- \\
.90 \\
1.1 \\
1.1 \\
2.3 \\
1.4 \\
--\end{array}$ & $\begin{array}{c}4.1 \\
-. \\
3.9 \\
-- \\
4.2 \\
3.8 \\
\overline{--} \\
4.6 \\
3.3 \\
--\end{array}$ & $\begin{array}{r}.190 \\
- \\
-160 \\
-- \\
.200 \\
.170 \\
-- \\
.470 \\
- \\
.370 \\
--\end{array}$ & $\begin{array}{r}.120 \\
-- \\
.110 \\
-- \\
.110 \\
.060 \\
-- \\
.150 \\
-- \\
.150 \\
--\end{array}$ \\
\hline $\begin{array}{l}\text { APR } \\
01 \ldots \\
01 \ldots\end{array}$ & $.02 \overline{0}$ & $2.10^{--}$ & $.12 \overline{0}$ &.$\overline{71}$ &.$\overline{83}$ & $2 . \overline{9}^{--}$ & $.1 \overline{40}$ & $.10 \overline{0}$ \\
\hline $\begin{array}{l}\text { MAY } \\
25 \ldots\end{array}$ & .030 & 1.30 & .090 & 1.1 & 1.1 & 2.5 & .210 & .050 \\
\hline $\begin{array}{r}\text { JUN } \\
28 \ldots \\
29 \ldots \\
29 \ldots \\
29 \ldots \\
29 \ldots \\
29 \ldots \\
30 \ldots \\
30 \ldots \\
30 \ldots \\
30 \ldots\end{array}$ & $\begin{array}{r}.050 \\
.080 \\
-- \\
.090 \\
-- \\
.090 \\
.090 \\
- \\
.090 \\
.080\end{array}$ & $\begin{array}{c}.590 \\
.860 \\
1.50 \\
-- \\
1.20 \\
1.70 \\
=- \\
1.40 \\
1.40\end{array}$ & $\begin{array}{r}.200 \\
.250 \\
-- \\
.150 \\
-- \\
.120 \\
.140 \\
-- \\
.150 \\
.120\end{array}$ & $\begin{array}{c}1.5 \\
3.2 \\
-- \\
1.8 \\
-- \\
1.4 \\
1.1 \\
=-- \\
1.1 \\
1.1\end{array}$ & $\begin{array}{c}1.7 \\
3.4 \\
1.9 \\
=- \\
1.5 \\
1.3 \\
=- \\
1.3 \\
1.1\end{array}$ & $\begin{array}{c}2.3 \\
4.3 \\
3.4 \\
-- \\
2.7 \\
3.0 \\
-- \\
2.7 \\
2.6\end{array}$ & $\begin{array}{r}.310 \\
.610 \\
-- \\
.380 \\
-- \\
.270 \\
.270 \\
-- \\
.230 \\
.220\end{array}$ & $\begin{array}{r}.110 \\
.140 \\
. \\
.140 \\
.- \\
.100 \\
.120 \\
-- \\
.100 \\
.090\end{array}$ \\
\hline $\begin{array}{l}\text { JUL } \\
01 \ldots \\
01 \ldots\end{array}$ & .070 & 1.40 & .140 & 1.3 & 1.4 & 2.8 & .270 & .100 \\
\hline $\begin{array}{l}\text { AUG } \\
08 \ldots\end{array}$ & .010 & .170 & .140 & .78 & .92 & 1.1 & .190 & .060 \\
\hline
\end{tabular}


Table 12. Water and bed-material analyses from Onion River at Hingham, 1979 and 1980 water years-Continued

\begin{tabular}{|c|c|c|c|c|c|c|c|c|c|}
\hline DATE & TIME & $\begin{array}{l}\text { STREAM- } \\
\text { FLOW, } \\
\text { INSTAN- } \\
\text { TANEOUS } \\
\text { (CFS) }\end{array}$ & $\begin{array}{c}\text { ALKA- } \\
\text { LINITY } \\
\text { FIELD } \\
\text { (MG/L } \\
\text { AS } \\
\text { CACO3) }\end{array}$ & $\begin{array}{l}\text { CHLO } \\
\text { RIDE } \\
\text { DIS- } \\
\text { SOLV } \\
\text { (MG/ } \\
\text { AS C }\end{array}$ & $\begin{array}{l}\text { O- } \\
\text { E, } \\
\text { VED } \\
\text { /L } \\
\text { CL) }\end{array}$ & $\begin{array}{l}\text { SOLIDS, } \\
\text { RESIDUE } \\
\text { AT } 180 \\
\text { DEG. C } \\
\text { DIS- } \\
\text { SOLVED } \\
\text { (MG/L) }\end{array}$ & $\begin{array}{c}\text { SOLIDS, } \\
\text { DIS- } \\
\text { SOLVED } \\
\text { (TONS } \\
\text { PER } \\
\text { AC-FT) }\end{array}$ & $\begin{array}{c}\text { SOLIDS, } \\
\text { DIS - } \\
\text { SOLVED } \\
\text { (TONS } \\
\text { PER } \\
\text { DAY) }\end{array}$ & $\begin{array}{c}\text { NITRO- } \\
\text { GEN, } \\
\text { NITRATE } \\
\text { TOTAL } \\
\text { (MG/L } \\
\text { AS N) }\end{array}$ \\
\hline \multicolumn{10}{|c|}{ AUG , 1979} \\
\hline $09 \ldots$ & 2215 & 133 & -- & & -- & -- & -- & -- & .470 \\
\hline $\begin{array}{l}09 \ldots \\
10 \ldots\end{array}$ & $\begin{array}{l}2315 \\
0015\end{array}$ & $\begin{array}{l}204 \\
255\end{array}$ & 162 & 14 & -- & 242 & .33 & 133 & .540 \\
\hline $\begin{array}{l}10 \ldots \\
10 \ldots\end{array}$ & $\begin{array}{l}0115 \\
0315\end{array}$ & $\begin{array}{l}285 \\
264\end{array}$ & $\begin{array}{l}189 \\
126\end{array}$ & $\begin{array}{l}13 \\
11\end{array}$ & & $\begin{array}{l}245 \\
188\end{array}$ & $\begin{array}{r}.33 \\
.26\end{array}$ & $\begin{array}{l}189 \\
134\end{array}$ & \\
\hline $10 \ldots$ & 0415 & 209 & $=$ & & -. & -- & $=$ & -- & 1.03 \\
\hline 10 & 081 & 117 & - & & - & -- & -- & - & 1.26 \\
\hline $\begin{array}{l}10 \ldots \\
12 \ldots\end{array}$ & $\begin{array}{l}1915 \\
1045\end{array}$ & $\begin{array}{r}137 \\
44\end{array}$ & $\overline{17}$ & 14 & - & $2 \overline{6}$ &.$\overline{36}$ & $31^{--}$ & 1.23 \\
\hline 12. & 1115 & 43 & -- & & - & - & $\ldots$ & -- & .670 \\
\hline 20. & 084 & 43 & - & & -- & $=-$ & $\bar{x}$ & & .000 \\
\hline 20 . & 0930 & 46 & 243 & 23 & & 329 & .45 & 41 & 120 \\
\hline 20 . & 1230 & 5 & -- & & $\cdots$ & -- & $-\bar{c}$ & $7^{--}$ & 1.29 \\
\hline $\begin{array}{l}20 \\
20\end{array}$ & $\begin{array}{l}1330 \\
2330\end{array}$ & $\begin{array}{l}52 \\
48\end{array}$ & $\begin{array}{l}261 \\
261\end{array}$ & $\begin{array}{l}23 \\
20\end{array}$ & & $\begin{array}{l}337 \\
335\end{array}$ & $\begin{array}{l}.46 \\
.46\end{array}$ & $\begin{array}{l}47 \\
43\end{array}$ & - \\
\hline & 003 & i & $=$ & & - & -- & $=$ & -. & 1.29 \\
\hline & 1130 & 5 & 243 & 21 & & 318 & .43 & 45 & \\
\hline
\end{tabular}

$\begin{array}{ccccccccc} & \text { NITRO- } & \text { NITRO- } & \text { NITRO- } & \text { NITRO- } & \text { NITRO- } & \text { GEN, } & & \text { PMOS- } \\ & \text { GEN, } & \text { GEN, } & \text { GEN, } & \text { GEN, } & \text { MONIA } & \text { NITRO- } & \text { PHOS- } & \text { PHORUS, } \\ & \text { NITRITE } & \text { NO2+NO3 } & \text { AMMONIA } & \text { ORGANIC } & \text { ORGANIC } & \text { GEN, } & \text { PHORUS, } & \text { ORTHO, } \\ & \text { TOTAL } & \text { TOTAL } & \text { TOTAL } & \text { TOTAL } & \text { TOTAL } & \text { TOTAL } & \text { TOTAL } & \text { TOTAL } \\ \text { DATE } & \text { (MG/L } & \text { (MG/L } & \text { (MG/L } & \text { (MG/L } & \text { (MG/L } & \text { (MG/L } & \text { (MG/L } & \text { (MG/L } \\ & \text { AS N) } & \text { AS N) } & \text { AS N) } & \text { AS N) } & \text { AS N) } & \text { AS N) } & \text { AS P) } & \text { AS P) }\end{array}$

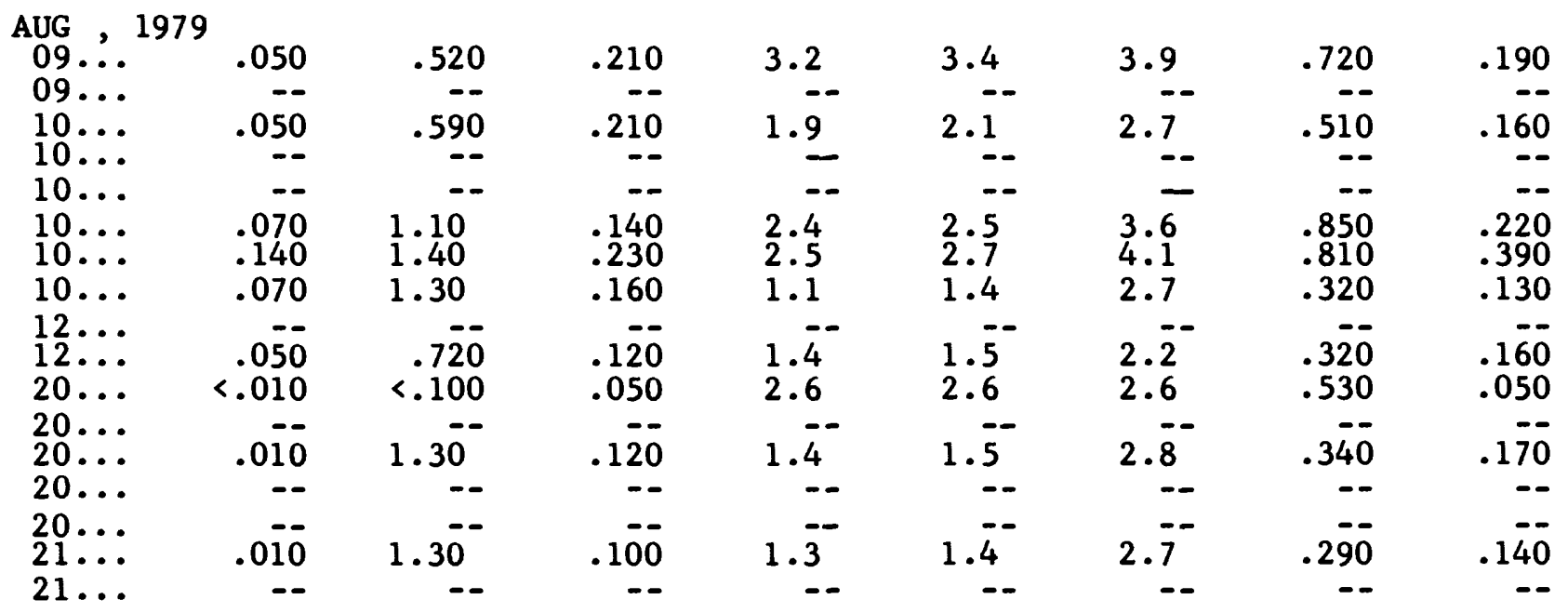


Table 12. Water and bed-material analyses from Onion River at Hingham, 1979 and 1980 water years-Continued

\begin{tabular}{|c|c|c|c|c|c|c|c|}
\hline DATE & TIME & $\begin{array}{l}\text { STREAM- } \\
\text { FLOW, } \\
\text { INSTAN- } \\
\text { TANEOUS } \\
\text { (CFS) }\end{array}$ & $\begin{array}{c}\text { PH } \\
\text { (STAND- } \\
\text { ARD } \\
\text { UNITS) }\end{array}$ & $\begin{array}{c}\text { OXYGEN, } \\
\text { DIS- } \\
\text { SOLVED } \\
\text { (MG/L) }\end{array}$ & $\begin{array}{l}\text { COLI- } \\
\text { FORM, } \\
\text { FECAL, } \\
0.7 \\
\text { UM-MF } \\
\text { (COLS./ } \\
100 \mathrm{ML})\end{array}$ & $\begin{array}{c}\text { STREP- } \\
\text { TOCOCCI } \\
\text { FECAL, } \\
\text { KF AGAR } \\
\text { (COLS. } \\
\text { PER } \\
100 \mathrm{ML})\end{array}$ & $\begin{array}{c}\text { OXYGEN } \\
\text { DEMAND, } \\
\text { BIO- } \\
\text { CHEM- } \\
\text { ICAL , } \\
5 \text { DAY } \\
(M G / L)\end{array}$ \\
\hline $\begin{array}{c}\text { DEC }, \\
05 \ldots \\
06 \ldots\end{array}$ & $\begin{array}{r}1978 \\
1620 \\
1915\end{array}$ & $\begin{array}{l}20 \\
20\end{array}$ & $\begin{array}{r}8.0 \\
--\end{array}$ & 13.2 & $4 \overline{30}$ & $2 \overline{0}$ & $3 . \overline{9}$ \\
\hline $\begin{array}{c}\text { JAN }, \\
17 \ldots \\
17 \ldots \\
17 \ldots\end{array}$ & $\begin{array}{r}19790930 \\
1230 \\
1700\end{array}$ & $\begin{array}{l}12 \\
12 \\
12\end{array}$ & $\begin{array}{r}7.6 \\
7.0 \\
-.-\end{array}$ & $\begin{array}{l}-- \\
-- \\
--\end{array}$ & $\overline{--}$ & $\begin{array}{l}-- \\
-- \\
39\end{array}$ & $\begin{array}{l}-- \\
1.1\end{array}$ \\
\hline $\begin{array}{l}\text { FEB } \\
27 \ldots \\
28 \ldots\end{array}$ & $\begin{array}{l}1600 \\
1230\end{array}$ & $\begin{array}{l}18 \\
18\end{array}$ & 8.1 & $\begin{array}{r}11.4 \\
--\end{array}$ & $1 \overline{10}$ & $\overline{57}$ & $3 . \overline{7}$ \\
\hline$\underset{23 \ldots}{\text { MAR }}$ & 1330 & 333 & -- & -- & 210 & 3300 & 2.2 \\
\hline $\begin{array}{l}\text { MAY } \\
25 \ldots \\
25 \ldots\end{array}$ & $\begin{array}{l}1015 \\
1600\end{array}$ & $\begin{array}{l}25 \\
26\end{array}$ & $=$ & $\begin{array}{r}10.2 \\
--\end{array}$ & $\overline{46}$ & $\overline{57}$ & 3.8 \\
\hline $\begin{array}{l}\text { JUN } \\
28 \ldots \\
28 \ldots\end{array}$ & $\begin{array}{l}0930 \\
1700\end{array}$ & $\begin{array}{l}13 \\
13\end{array}$ & $\begin{array}{r}8.3 \\
--\end{array}$ & $\begin{array}{r}6.3 \\
--\end{array}$ & 250 & 310 & $\begin{array}{r}5.5 \\
--\end{array}$ \\
\hline $\begin{array}{l}\text { AUG } \\
08 \ldots \\
08 \ldots\end{array}$ & $\begin{array}{l}1445 \\
1800\end{array}$ & $\begin{array}{l}13 \\
13\end{array}$ & 8.3 & -- & 180 & 180 & $4 . \overline{4}$ \\
\hline $\begin{array}{l}\text { SEP } \\
14 \ldots \\
14 \ldots \\
14 \ldots\end{array}$ & $\begin{array}{l}1015 \\
1355 \\
1600\end{array}$ & $\begin{array}{l}12 \\
13 \\
13\end{array}$ & $\begin{array}{r}8.1 \\
-- \\
--\end{array}$ & $\begin{array}{l}-- \\
7.5 \\
-.\end{array}$ & E390 & $\begin{array}{l}-- \\
-- \\
73\end{array}$ & $\begin{array}{r}-- \\
4.0 \\
--\end{array}$ \\
\hline
\end{tabular}


Table 12. Water and bed-material analyses from Onion River at Hingham, 1979 and 1980 water years--Continued

\begin{tabular}{|c|c|c|c|c|c|c|c|c|c|}
\hline DATE & TIME & $\begin{array}{l}\text { STREAM- } \\
\text { FLOW, } \\
\text { INSTAN- } \\
\text { TANEOUS } \\
\text { (CFS) }\end{array}$ & $\begin{array}{c}\text { ALKA- } \\
\text { LINITY } \\
\text { FIELD } \\
\text { (MG/L } \\
\text { AS } \\
\text { CACO3) }\end{array}$ & $\begin{array}{l}\text { CHLO- } \\
\text { RIDE, } \\
\text { DIS- } \\
\text { SOLVED } \\
\text { (MG/L } \\
\text { AS CL) }\end{array}$ & $\begin{array}{l}\text { SOLIDS, } \\
\text { RESIDUE } \\
\text { AT } 180 \\
\text { DEG.C } \\
\text { DIS- } \\
\text { SOLVED } \\
\text { (MG/L) }\end{array}$ & $\begin{array}{c}\text { SOLIDS, } \\
\text { DIS- } \\
\text { SOLVED } \\
\text { (TONS } \\
\text { PER } \\
\text { AC-FT) }\end{array}$ & $\begin{array}{c}\text { SOLIDS, } \\
\text { DIS - } \\
\text { SOLVED } \\
\text { (TONS } \\
\text { PER } \\
\text { DAY) }\end{array}$ & $\begin{array}{l}\text { NITRO- } \\
\text { GEN, } \\
\text { NITRATE } \\
\text { TOTAL } \\
\text { (MG/L } \\
\text { AS N) }\end{array}$ & $\begin{array}{c}\text { NITRO- } \\
\text { GEN, } \\
\text { NITRITE } \\
\text { TOTAL } \\
\text { (MG/L } \\
\text { AS N) }\end{array}$ \\
\hline $\begin{array}{c}\text { Nov }, \\
16 . .\end{array}$ & ${ }^{9} 0830$ & 19 & 290 & 18 & 362 & .49 & 19 & 1.49 & .010 \\
\hline & 1100 & 14 & 290 & 21 & 360 & .49 & 14 & 1.60 & .000 \\
\hline $\begin{array}{r}\text { JAN }, \\
17 \ldots \\
17 \ldots \\
17 \ldots \\
17 \ldots \\
17 \ldots \\
17 \ldots \\
24 \ldots \\
24 \ldots\end{array}$ & $\begin{array}{l}0 \\
0815 \\
0915 \\
1415 \\
1515 \\
2015 \\
2115 \\
1100 \\
1130\end{array}$ & $\begin{array}{r}\text { E68 } \\
\text { E68 } \\
\text { E68 } \\
\text { E68 } \\
\text { E68 } \\
\text { E68 } \\
11 \\
11\end{array}$ & $\begin{array}{r}1 \overline{-} \\
\overline{-} \\
126 \\
\overline{-} \\
122 \\
306\end{array}$ & $\begin{array}{l}20 \\
19 \\
18 \\
18 \\
25\end{array}$ & $\begin{array}{r}--- \\
258 \\
-\overline{-} \\
237 \\
-- \\
232 \\
--- \\
400\end{array}$ & $\begin{array}{l}--- \\
.35 \\
.-- \\
.32 \\
-- \\
.32 \\
.-- \\
.54\end{array}$ & $\begin{array}{l}=- \\
=- \\
=- \\
=-\end{array}$ & $\begin{array}{c}1.85 \\
1.75^{--} \\
1.45^{--} \\
2.97^{--} \\
--\end{array}$ & $\begin{array}{r}.050 \\
.050 \\
.-- \\
.050 \\
.030 \\
--\end{array}$ \\
\hline $\begin{array}{c}\text { MAR } \\
17 \ldots \\
17 \ldots \\
17 \ldots \\
17 \ldots \\
18 \ldots \\
18 \ldots \\
18 \ldots \\
27 \ldots\end{array}$ & $\begin{array}{l}0945 \\
1045 \\
2145 \\
2245 \\
0345 \\
0445 \\
0845 \\
1200\end{array}$ & $\begin{array}{r}\text { E24 } \\
\text { E27 } \\
\text { E40 } \\
\text { E50 } \\
\text { E70 } \\
\text { E70 } \\
57 \\
39\end{array}$ & $\begin{array}{r}126 \\
\overline{75} \\
-- \\
82 \\
-- \\
108 \\
230\end{array}$ & $\begin{array}{ll}23 & -- \\
21 & -- \\
22 & - \\
15 & - \\
21 & \end{array}$ & $\begin{array}{r}218 \\
16- \\
162 \\
185 \\
-- \\
167 \\
325\end{array}$ & $\begin{array}{l}.30 \\
.-22 \\
.- \\
.25 \\
-- \\
.23 \\
.44\end{array}$ & $\begin{array}{l}-- \\
=- \\
=- \\
26 \\
34\end{array}$ & $\begin{array}{l}1.35^{--} \\
1.35 \\
1.36^{--} \\
1.66 \\
1.67\end{array}$ & $\begin{array}{l}.050 \\
-- \\
.050 \\
.040 \\
.040 \\
.030\end{array}$ \\
\hline $\begin{array}{r}\text { APR } \\
08 \ldots \\
08 \ldots \\
08 \ldots \\
08 \ldots \\
08 \ldots \\
08 \ldots \\
08 \ldots \\
08 \ldots \\
08 \ldots \\
09 \ldots \\
09 \ldots \\
10 \ldots\end{array}$ & $\begin{array}{l}0730 \\
0830 \\
0930 \\
1030 \\
1130 \\
1430 \\
2000 \\
2030 \\
2130 \\
0130 \\
1930 \\
1130\end{array}$ & $\begin{array}{l}143 \\
191 \\
333 \\
348 \\
348 \\
253 \\
112 \\
140 \\
174 \\
218 \\
195 \\
148\end{array}$ & $\begin{array}{r}180 \\
-- \\
189 \\
-\overline{-} \\
-- \\
162 \\
=- \\
-- \\
--\end{array}$ & $\begin{array}{ll}21 & \\
21 & -- \\
20 & -- \\
& -- \\
20 & - \\
& -- \\
-- & - \\
--\end{array}$ & $\begin{array}{r}294 \\
-- \\
301 \\
--- \\
296 \\
-- \\
-- \\
300 \\
-- \\
-- \\
-- \\
--\end{array}$ & $\begin{array}{r}.40 \\
. \overline{-41} \\
. \overline{-} \\
.40 \\
=- \\
. \overline{.41} \\
-- \\
-- \\
-- \\
--\end{array}$ & $\begin{array}{r}114 \\
271 \\
278^{--} \\
113^{--} \\
=- \\
=- \\
--\end{array}$ & $\begin{array}{c}1.86^{--} \\
2.16^{--} \\
2.35^{--} \\
2.25 \\
2.25^{--} \\
2.15 \\
2.45 \\
2.35\end{array}$ & $\begin{array}{r}.040 \\
-- \\
.040 \\
-- \\
.050 \\
.050 \\
-- \\
.050 \\
.050 \\
.050 \\
.050\end{array}$ \\
\hline $\begin{array}{r}\text { MAY } \\
08 .\end{array}$ & 1010 & 16 & 300 & 22 & -- & -- & -- & .390 & .020 \\
\hline
\end{tabular}


Table 12. Water and bed-material analyses from Onion River at Hingham, 1979 and 1980 water years--Continued

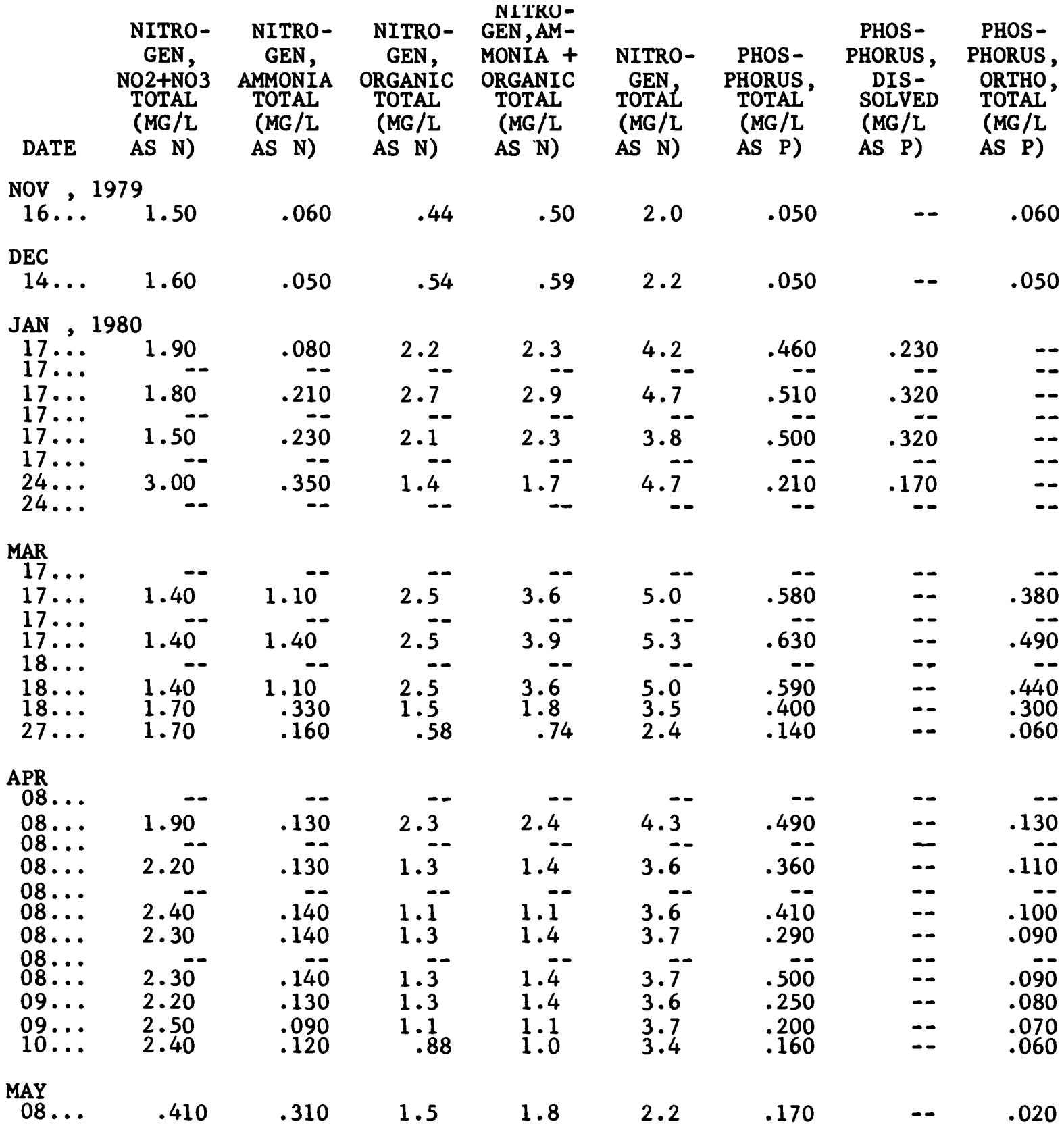


Table 12. Water and bed-material analyses from Onion River at Hingham, 1979 and 1980 water years--Continued

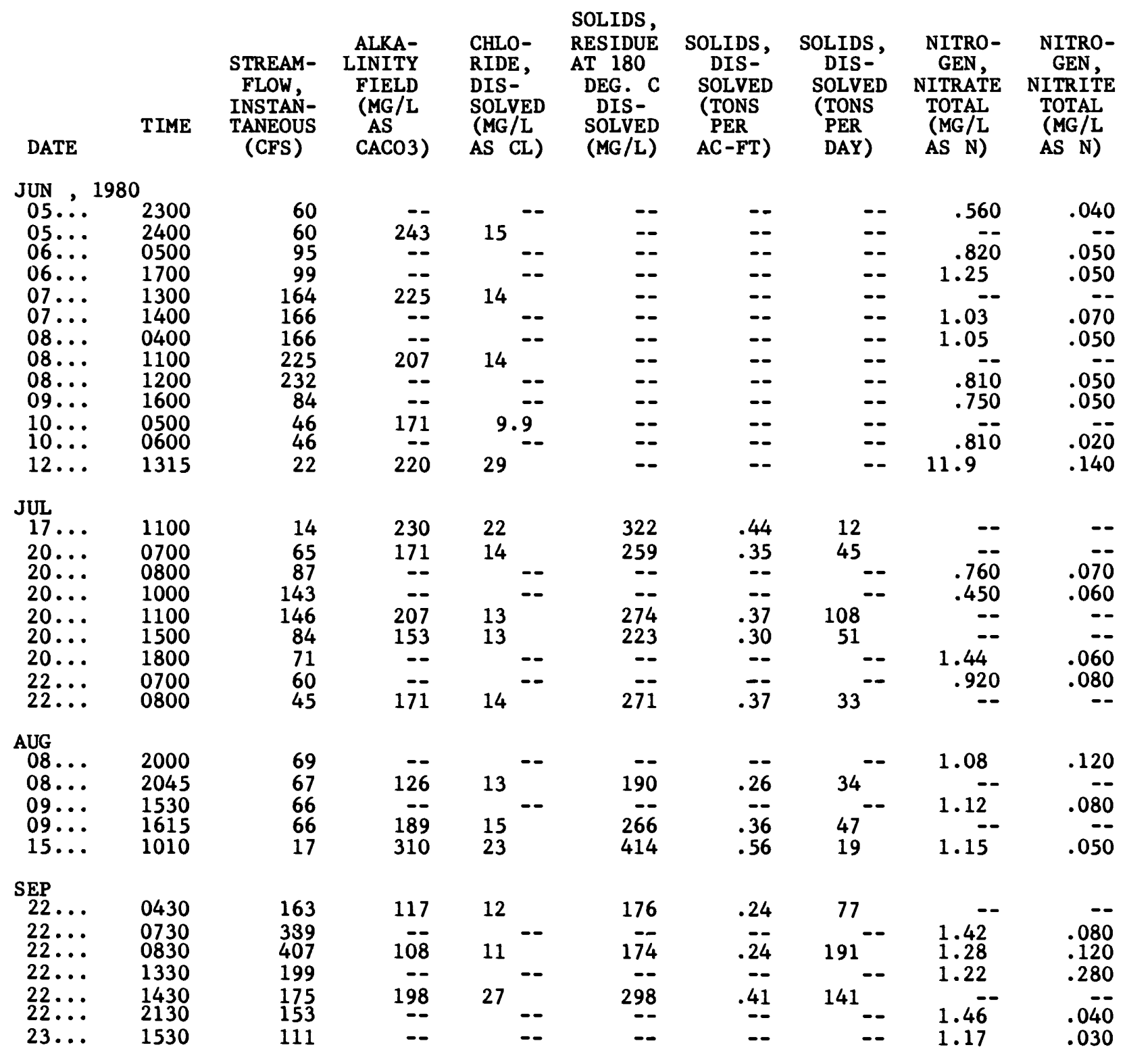


Table 12. Water and bed-material analyses from Onion River at Hingham, 1979 and 1980 water years--Continued

\begin{tabular}{|c|c|c|c|c|c|c|c|c|}
\hline DATE & $\begin{array}{c}\text { NITRO- } \\
\text { GEN, } \\
\text { NO2+NO3 } \\
\text { TOTAL } \\
\text { (MG/L } \\
\text { AS N) }\end{array}$ & $\begin{array}{l}\text { NITRO- } \\
\text { GEN, } \\
\text { AMMONIA } \\
\text { TOTAL } \\
\text { (MG/L } \\
\text { AS N) }\end{array}$ & $\begin{array}{l}\text { NITRO- } \\
\text { GEN, } \\
\text { ORGANIC } \\
\text { TOTAL } \\
\text { (MG/L } \\
\text { AS N) }\end{array}$ & $\begin{array}{l}\text { NITRO- } \\
\text { GEN, AM- } \\
\text { MONIA + } \\
\text { ORGANIC } \\
\text { TOTAL } \\
\text { (MG/L } \\
\text { AS N) }\end{array}$ & $\begin{array}{l}\text { NITRO- } \\
\text { GEN, } \\
\text { TOTAL } \\
\text { (MG/L } \\
\text { AS N) }\end{array}$ & $\begin{array}{c}\text { PHOS- } \\
\text { PHORUS, } \\
\text { TOTAL } \\
\text { (MG/L } \\
\text { AS P) }\end{array}$ & $\begin{array}{c}\text { PHOS- } \\
\text { PHORUS, } \\
\text { DIS- } \\
\text { SOLVED } \\
\text { (MG/L } \\
\text { AS P) }\end{array}$ & $\begin{array}{c}\text { PHOS - } \\
\text { PHORUS, } \\
\text { ORTHO, } \\
\text { TOTAL } \\
\text { (MG/L } \\
\text { AS P) }\end{array}$ \\
\hline \multicolumn{9}{|c|}{ JUN , 1980} \\
\hline $05 \ldots$ & .600 & .020 & 1.5 & 1.5 & 2.1 & .240 & -- & .040 \\
\hline $06 \ldots$ & $\begin{array}{l}.870 \\
1.30\end{array}$ & $\begin{array}{l}.07 \overline{0} \\
.070\end{array}$ & $\begin{array}{l}1.7 \\
1.1\end{array}$ & $\begin{array}{l}1.8 \\
1.1\end{array}$ & $\begin{array}{l}2.7 \\
2.5\end{array}$ & $\begin{array}{l}.380 \\
.220\end{array}$ & $=$ & $\begin{array}{l}.110 \\
.060\end{array}$ \\
\hline $07 \ldots$ & & & 1 & $\ldots$ & & & -- & - \\
\hline $\begin{array}{l}07 \ldots \\
08 \ldots\end{array}$ & $\begin{array}{l}1.10 \\
1.10\end{array}$ & $\begin{array}{l}.130 \\
.100\end{array}$ & $\begin{array}{l}1.3 \\
1.3\end{array}$ & $\begin{array}{l}1.4 \\
1.4\end{array}$ & $\begin{array}{l}2.5 \\
2.5\end{array}$ & $\begin{array}{l}.220 \\
.240\end{array}$ & -- & $\begin{array}{l}.050 \\
.090\end{array}$ \\
\hline $08 \ldots$ & -- & -- & & -- & -- & -- & -- & -- \\
\hline $\begin{array}{l}08 \ldots \\
09 \ldots\end{array}$ & $\begin{array}{l}.860 \\
.800\end{array}$ & $\begin{array}{l}.110 \\
.090\end{array}$ & $\begin{array}{l}1.3 \\
1.3\end{array}$ & $\begin{array}{l}1.4 \\
1.4\end{array}$ & $\begin{array}{l}2.3 \\
2.2\end{array}$ & $\begin{array}{r}.280 \\
.280\end{array}$ & $=$ & .090 \\
\hline $10 \ldots$ & & & & & & & -- & - \\
\hline $\begin{array}{l}10 \ldots \\
12 \ldots\end{array}$ & 12.830 & $\begin{array}{l}.090 \\
.140\end{array}$ & $\begin{array}{l}1.3 \\
2.1\end{array}$ & $\begin{array}{l}1.4 \\
2.2\end{array}$ & $\begin{array}{l}2.2 \\
14\end{array}$ & $\begin{array}{l}.230 \\
.400\end{array}$ & -- & $\begin{array}{l}.080 \\
.180\end{array}$ \\
\hline \multicolumn{9}{|l|}{ JUL } \\
\hline $17 \ldots$ & -- & -- & -- & -- & -- & .000 & -- & -- \\
\hline $20 \ldots$ & -- & -- & -- & -- & -- & -- & -- & -- \\
\hline $20 \ldots$ & .830 & .140 & 3.1 & 3.2 & 4.0 & .900 & -- & .180 \\
\hline 20 . & .510 & .120 & 1.8 & 1.9 & 2.4 & .380 & -- & .120 \\
\hline 20 . & -- & -- & -- & -- & -- & -- & -- & -- \\
\hline $20 \ldots$ & -- & -- & -- & -- & -- & -- & -- & -- \\
\hline $20 \ldots$ & 1.50 & .070 & 1.1 & 1.1 & 2.7 & .420 & -- & .180 \\
\hline $22 \ldots$ & - & 0 & 1.0 & 1.0 & 2.0 & 0 & - & 0 \\
\hline \multicolumn{9}{|l|}{ AUG } \\
\hline 08 & 1.20 & .290 & .91 & 1.1 & 2.4 & .480 & -- & .480 \\
\hline 08. & & $=-$ & -- & $=-$ & $2^{--}$ & $\overline{0}$ & -- & 320 \\
\hline & 1.20 & .230 & 1.4 & 1.6 & 2.8 & .400 & - & .320 \\
\hline $15 \ldots$ & 1.20 & 040 & 1 & 1 & $2 .-$ & 270 & $\ldots$ & 140 \\
\hline \multirow{2}{*}{\multicolumn{9}{|c|}{ SEP }} \\
\hline & & & & & & & & \\
\hline $\begin{array}{l}22 \ldots \\
22 \ldots\end{array}$ & $1.50^{--}$ & $1 \overline{40}$ & $\overline{8}^{--}$ & $0^{--}$ & $3.4^{-}$ & $86 \overline{0}$ & $=-$ & 330 \\
\hline $22 \ldots$ & 1.40 & .220 & 3.0 & 3.2 & $\begin{array}{l}2.4 \\
4.6\end{array}$ & $\begin{array}{l}.000 \\
.990\end{array}$ & - & .440 \\
\hline 22 & 1.50 & .180 & 2.5 & 2.7 & 4.2 & .350 & -- & .010 \\
\hline $22 \ldots$ & $1.50^{--}$ & .080 & $1.1^{--}$ & $1 . \overline{1}$ & $27^{--}$ & 380 & -- & 140 \\
\hline 23 . & 1.20 & .070 & 1.1 & 1.1 & 2.4 & .330 & -- & .170 \\
\hline
\end{tabular}


Table 12. Water and bed-material analyses from Onion River at Hingham, 1979 and 1980 water years--Continued

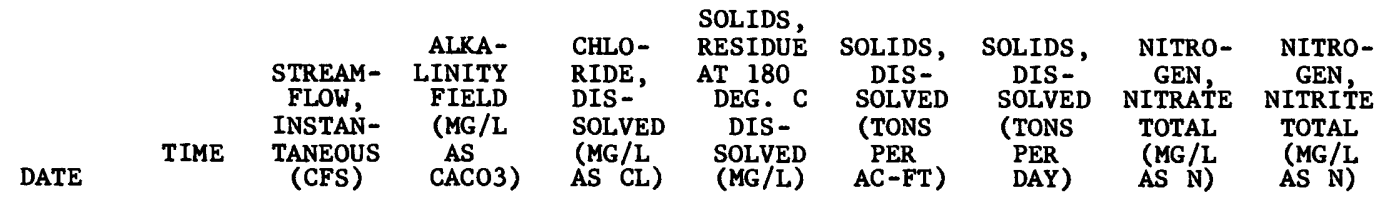

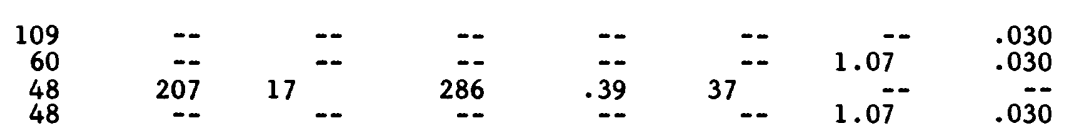

\begin{tabular}{|c|c|c|c|c|c|c|c|c|}
\hline & $\begin{array}{c}\text { NITRO- } \\
\text { GEN, } \\
\text { NO2+NO3 } \\
\text { TOTAL } \\
\text { (MG/L }\end{array}$ & $\begin{array}{l}\text { NITRO- } \\
\text { GEN, } \\
\text { AMMONIA } \\
\text { TOTAL } \\
\text { (MG/L }\end{array}$ & $\begin{array}{l}\text { NITRO- } \\
\text { GEN, } \\
\text { ORGANIC } \\
\text { TOTAL } \\
\text { (MG/L }\end{array}$ & $\begin{array}{l}\text { NITRO- } \\
\text { GEN, AM- } \\
\text { MONIA + } \\
\text { ORGANIC } \\
\text { TOTAL } \\
\text { (MG/L }\end{array}$ & $\begin{array}{l}\text { NITRO- } \\
\text { GEN, } \\
\text { TOTAL } \\
\text { (MG/L }\end{array}$ & $\begin{array}{c}\text { PHOS- } \\
\text { PHORUS, } \\
\text { TOTAL } \\
\text { (MG/L }\end{array}$ & $\begin{array}{c}\text { PHOS - } \\
\text { PHORUS, } \\
\text { DIS- } \\
\text { SOLVED } \\
\text { (MG/L }\end{array}$ & $\begin{array}{c}\text { PHOS - } \\
\text { PHORUS, } \\
\text { ORTHO, } \\
\text { TOTAL } \\
\text { (MG/L }\end{array}$ \\
\hline DATE & AS $N$ ) & AS $\mathbf{N}$ ) & AS $N$ ) & AS $N$ ) & AS N) & AS P) & AS P) & AS P) \\
\hline
\end{tabular}

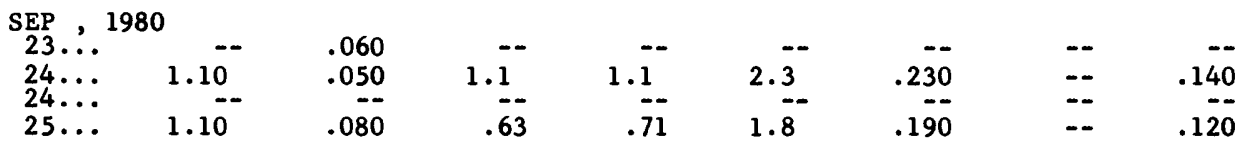

\begin{tabular}{|c|c|c|c|c|c|c|c|c|c|c|c|}
\hline DATE & TIME & $\begin{array}{l}\text { STREAM- } \\
\text { FLOW, } \\
\text { INSTAN- } \\
\text { TANEOUS } \\
\text { (CFS) }\end{array}$ & $\begin{array}{c}\text { PH } \\
\text { (STAND- } \\
\text { ARD } \\
\text { UNITS) }\end{array}$ & $\begin{array}{l}\text { TEMPER- } \\
\text { ATURE } \\
\text { (DEG C) }\end{array}$ & $\begin{array}{r}\text { TUR } \\
\text { BID } \\
\text { ITY } \\
\text { (MG/I } \\
\text { AS } \\
\text { SIO2 }\end{array}$ & & $\begin{array}{c}\text { OXYGEN, } \\
\text { DIS- } \\
\text { SOLVED } \\
\text { (MG/L) }\end{array}$ & $\begin{array}{c}\text { OXYGEN, } \\
\text { DIS - } \\
\text { SOLVED } \\
\text { (PER- } \\
\text { CENT } \\
\text { SATUR- } \\
\text { ATION) }\end{array}$ & $\begin{array}{l}\text { COLI - } \\
\text { FORM, } \\
\text { FECAL, } \\
0.7 \\
\text { UM-MF } \\
\text { (COLS./ } \\
100 \mathrm{ML} \text { ) }\end{array}$ & $\begin{array}{c}\text { STREP- } \\
\text { TOCOCCI } \\
\text { FECAL, } \\
\text { KF AGAR } \\
\text { (COLS. } \\
\text { PER } \\
100 \mathrm{ML})\end{array}$ & $\begin{array}{l}\text { OXYGEN } \\
\text { DEMAND, } \\
\text { BIO- } \\
\text { CHEM- } \\
\text { ICAL, } \\
5 \text { DAY } \\
\text { (MG/L) }\end{array}$ \\
\hline $\begin{array}{c}\text { NOV , } \\
15 \ldots \\
16 \ldots\end{array}$ & $\begin{array}{r}1979 \\
1030 \\
1245\end{array}$ & $\begin{array}{l}17 \\
19\end{array}$ & $\begin{array}{r}8.4 \\
--\end{array}$ & $\begin{array}{r}4.5 \\
--\end{array}$ & & -- & $\begin{array}{r}13.0 \\
--\end{array}$ & $\begin{array}{r}105 \\
--\end{array}$ & $\overline{70}$ & 40 & 4.7 \\
\hline $\begin{array}{l}\text { DEC } \\
13 \ldots \\
14 \ldots\end{array}$ & $\begin{array}{l}1600 \\
1600\end{array}$ & $\begin{array}{l}20 \\
14\end{array}$ & $\begin{array}{c}8.3 \\
--\end{array}$ & $\begin{array}{c}3.0 \\
-\cdots\end{array}$ & & -- & $\begin{array}{c}13.6 \\
-.\end{array}$ & $\begin{array}{r}105 \\
\ldots-\end{array}$ & 120 & $\overline{\mathrm{K}} \overline{1}$ & 2.4 \\
\hline $\begin{array}{c}\text { JAN , } \\
24 \ldots \\
24 \ldots \\
26 \ldots\end{array}$ & $\begin{array}{r}1980 \\
1100 \\
1730 \\
1510\end{array}$ & $\begin{array}{l}11 \\
11 \\
11\end{array}$ & $\begin{array}{c}7.5 \\
--\end{array}$ & $\begin{array}{c}.5 \\
-- \\
--\end{array}$ & 5 & -- & $\begin{array}{r}10.1 \\
-- \\
--\end{array}$ & $\begin{array}{l}73 \\
-\infty \\
--\end{array}$ & K4 & -- & $\begin{array}{r}1.9 \\
- \\
--\end{array}$ \\
\hline $\begin{array}{l}\text { FEB } \\
02 \ldots \\
28 \ldots \\
28 \ldots \\
28 \ldots\end{array}$ & $\begin{array}{l}1510 \\
0750 \\
0855 \\
1345\end{array}$ & $\begin{array}{l}12 \\
13 \\
13 \\
13\end{array}$ & $\begin{array}{c}-- \\
6.9 \\
--\end{array}$ & $\begin{array}{l}-- \\
-- \\
--\end{array}$ & 14 & -- & 6.0 & 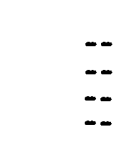 & $\overline{--}$ & $\overline{--}$ & 5. \\
\hline $\begin{array}{l}\text { MAR } \\
26 \ldots \\
27 \ldots\end{array}$ & $\begin{array}{l}1520 \\
1630\end{array}$ & $\begin{array}{l}28 \\
38\end{array}$ & -- & -- & 15 & $\sim$ & -- & -- & $\overline{73}$ & $4 \overline{70}$ & 3.5 \\
\hline $\begin{array}{c}\text { MAY } \\
07 \ldots \\
08 \ldots \\
08 \ldots \\
12 \ldots\end{array}$ & $\begin{array}{l}1145 \\
1010 \\
1430 \\
1740\end{array}$ & $\begin{array}{l}15 \\
16 \\
17 \\
14\end{array}$ & $\begin{array}{c}-- \\
\overline{--} \\
--\end{array}$ & $\begin{array}{r}12.5 \\
11.0 \\
-.\end{array}$ & 38 & -- & $\begin{array}{r}11.1 \\
-. \\
--\end{array}$ & $\begin{array}{c}109 \\
-- \\
--\end{array}$ & K15 & $\overline{48}$ & $\begin{array}{c}-- \\
6.3 \\
--\end{array}$ \\
\hline $\begin{array}{r}\text { JUN } \\
12 \ldots \\
12 \ldots \\
27 \ldots\end{array}$ & $\begin{array}{l}1315 \\
1630 \\
1930\end{array}$ & $\begin{array}{l}22 \\
21 \\
10\end{array}$ & $\begin{array}{c}8.1 \\
-- \\
--\end{array}$ & $\begin{array}{r}22.0 \\
\ldots \\
\ldots\end{array}$ & 25 & -- & $\begin{array}{c}8.0 \\
-- \\
--\end{array}$ & $\begin{array}{l}95 \\
-- \\
--\end{array}$ & 200 & 95 & $\begin{array}{r}3.2 \\
\ldots \\
\ldots\end{array}$ \\
\hline $\begin{array}{c}\text { JUL } \\
12 \ldots \\
16 \ldots \\
17 \ldots\end{array}$ & $\begin{array}{l}1845 \\
1540 \\
1610\end{array}$ & $13^{8.3}$ & $\begin{array}{l}-- \\
--\end{array}$ & $\begin{array}{r}-.- \\
--5\end{array}$ & 30 & -- & $\begin{array}{l}-- \\
--\end{array}$ & 95 & 830 & 270 & 6.0 \\
\hline $\begin{array}{l}\text { AUG } \\
02 \ldots \\
14 \ldots \\
15 \ldots \\
15 \ldots\end{array}$ & $\begin{array}{l}1600 \\
1430 \\
1010 \\
1330\end{array}$ & $\begin{array}{l}11 \\
20 \\
17 \\
17\end{array}$ & $\begin{array}{r}-. . \\
--\end{array}$ & $\begin{array}{r}-.- \\
24.0 \\
22.0 \\
-.\end{array}$ & 30 & -- & $\begin{array}{c}-- \\
8.1 \\
--\end{array}$ & $\begin{array}{c}-- \\
-- \\
--\end{array}$ & $\begin{array}{c}-- \\
-- \\
550\end{array}$ & $\begin{array}{l}-- \\
-- \\
48\end{array}$ & $\begin{array}{c}-- \\
5.4 \\
-.\end{array}$ \\
\hline
\end{tabular}


Table 12. Water and bed-material analyses from Onion River at Hingham, 1979 and 1980 water years--Continued

\begin{tabular}{|c|c|c|c|c|c|c|c|c|c|c|c|}
\hline DATE & TIME & $\begin{array}{l}\text { STREAM- } \\
\text { FLOW, } \\
\text { INSTAN- } \\
\text { TANEOUS } \\
\text { (CFS) }\end{array}$ & $\begin{array}{c}\text { CARBON, } \\
\text { ORGANIC } \\
\text { DIS- } \\
\text { SOLVED } \\
\text { (MG/L } \\
\text { AS C) }\end{array}$ & $\begin{array}{l}\text { CARBON, } \\
\text { ORGANIC } \\
\text { SUS- } \\
\text { PENDED } \\
\text { TOTAL } \\
\text { (MG/L } \\
\text { AS C) }\end{array}$ & $\begin{array}{l}\text { PERI- } \\
\text { PHYTON } \\
\text { BIOMASS } \\
\text { ASH } \\
\text { WEIGHT' } \\
\text { G/SQ M }\end{array}$ & $\begin{array}{c}\text { PERI- } \\
\text { PHYTON } \\
\text { BIOMASS } \\
\text { TOTAL } \\
\text { DRY } \\
\text { WEIGHT } \\
\text { G/SQ M }\end{array}$ & $\begin{array}{c}\text { CHLOR-A } \\
\text { PERI- } \\
\text { PHYTON } \\
\text { CHROMO- } \\
\text { GRAPHIC } \\
\text { FLUOROM } \\
\text { (MG/M2) }\end{array}$ & $\begin{array}{c}\text { CHLOR-B } \\
\text { PERI- } \\
\text { PHYTON } \\
\text { CHROMO- } \\
\text { GRAPHIC } \\
\text { FLUOROM } \\
\text { (MG/M2) }\end{array}$ & $\begin{array}{l}\text { BIOMASS } \\
\text { CHLORO- } \\
\text { PHYLL } \\
\text { RATIO } \\
\text { PERI- } \\
\text { PHYTON } \\
\text { (UNITS) }\end{array}$ & & \\
\hline $\begin{array}{c}\mathrm{DEC} \\
25 . .\end{array}$ & $0_{1500}^{1979}$ & 92 & -- & .60 & -- & -- & -- & -- & -- & & \\
\hline $\begin{array}{l}\text { JUL } \\
17 \ldots \\
22 \ldots\end{array}$ & $\begin{array}{l}1100 \\
1325\end{array}$ & $\begin{array}{l}14 \\
37\end{array}$ & $13^{--}$ & $2 . \overline{8}$ & 9.92 & 14.6 & 35.5 & 5.13 & 132 & & \\
\hline$\underset{15}{\text { AUG }}$ & 1010 & 17 & -- & - & 10.6 & 13.0 & 15.1 & 1.54 & 159 & & \\
\hline DATE & TIME & $\begin{array}{l}\text { STREAM- } \\
\text { FLOW, } \\
\text { INSTAN- } \\
\text { TANEOUS } \\
\text { (CFS) }\end{array}$ & $\begin{array}{l}\text { ARSENIC } \\
\text { TOTAL } \\
\text { IN BOT- } \\
\text { TOM MA- } \\
\text { TERIAL } \\
\text { (UG/G } \\
\text { AS AS) }\end{array}$ & $\begin{array}{l}\text { CADMIUM } \\
\text { RECOV. } \\
\text { FM BOT- } \\
\text { TOM MA- } \\
\text { TERIAL } \\
\text { (UG/G } \\
\text { AS CD) }\end{array}$ & $\begin{array}{l}\text { CHRO- } \\
\text { MIUM, } \\
\text { RECOV. } \\
\text { FM BOT- } \\
\text { TOM MA- } \\
\text { TERIAL } \\
\text { (UG/G) }\end{array}$ & $\begin{array}{l}\text { COPPER, } \\
\text { RECOV. } \\
\text { FM BOT- } \\
\text { TOM MA- } \\
\text { TERIAL } \\
\text { (UG/G } \\
\text { AS CU) }\end{array}$ & $\begin{array}{c}\text { LEAD, } \\
\text { RECOV. } \\
\text { FM BOT- } \\
\text { TOM MA- } \\
\text { TERIAL } \\
\text { (UG/G } \\
\text { AS PB) }\end{array}$ & $\begin{array}{c}\text { MERCURY } \\
\text { RECOV. } \\
\text { FM BOT - } \\
\text { TOMCMA- } \\
\text { TERIAL } \\
\text { (UG/L } \\
\text { AS HG) }\end{array}$ & $\begin{array}{c}\text { NICKEL, } \\
\text { RECOV: } \\
\text { FM BOT- } \\
\text { TOM MA- } \\
\text { TERIAL } \\
\text { (UG/G } \\
\text { AS NI) }\end{array}$ & $\begin{array}{c}\text { ZINC, } \\
\text { RECOV. } \\
\text { FM BOT- } \\
\text { TOM MA- } \\
\text { TERIAL } \\
\text { (UG /G } \\
\text { AS ZN) }\end{array}$ & \\
\hline $\begin{array}{c}\text { AUG }, 1 \\
15 \ldots .\end{array}$ & $1980_{1010}$ & 17 & 0 & $<10$ & $<10$ & $<10$ & 10 & .00 & 10 & 9 & \\
\hline DATE & TIME & $\begin{array}{l}\text { STREAM- } \\
\text { FLOW, } \\
\text { INSTAN- } \\
\text { TANEOUS } \\
\text { (CFS) }\end{array}$ & $\begin{array}{l}\text { PCB, } \\
\text { TOTAL } \\
\text { IN BOT- } \\
\text { TOM MA- } \\
\text { TERIAL } \\
\text { (UG/KG) }\end{array}$ & $\begin{array}{l}\text { ALDRIN, } \\
\text { TOTAL } \\
\text { IN BOT- } \\
\text { TOM MA- } \\
\text { TERIAL } \\
\text { (UG/KG) }\end{array}$ & $\begin{array}{l}\text { CHLOR- } \\
\text { DANE, } \\
\text { TOTAL } \\
\text { IN BOT- } \\
\text { TOM MA- } \\
\text { TERIAL } \\
\text { (UG/KG) }\end{array}$ & $\begin{array}{c}\text { DDD, } \\
\text { TOTAL } \\
\text { IN BOT- } \\
\text { TOM MA- } \\
\text { TERIAL } \\
\text { (UG/KG) }\end{array}$ & $\begin{array}{l}\text { DDE, } \\
\text { TOTAL } \\
\text { IN BOT- } \\
\text { TOM MA- } \\
\text { TERIAL } \\
\text { (UG/KG) }\end{array}$ & $\begin{array}{l}\text { DDT, } \\
\text { TOTAL } \\
\text { IN BOT- } \\
\text { TOM MA- } \\
\text { TERIAL } \\
\text { (UG /KG) }\end{array}$ & $\begin{array}{c}\text { DI - } \\
\text { AZINON, } \\
\text { TOTAL } \\
\text { IN BOT- } \\
\text { TOM MA- } \\
\text { TERIAL } \\
\text { (UG/KG) }\end{array}$ & $\begin{array}{c}\text { DI- } \\
\text { ELDRIN, } \\
\text { TOTAL } \\
\text { IN BOT- } \\
\text { TOM MA- } \\
\text { TERIAL } \\
\text { (UG/KG) }\end{array}$ & $\begin{array}{c}\text { ENDRIN, } \\
\text { TOTAL } \\
\text { IN BOT- } \\
\text { TOM MA- } \\
\text { TERIAI } \\
\text { (UG/KG) }\end{array}$ \\
\hline $\begin{array}{c}\text { AUG }, 1 \\
15 \ldots\end{array}$ & ${ }_{1080}$ & 17 & .00 & .0 & .00 & .0 & .0 & .0 & .0 & .0 & . C \\
\hline DATE & $\begin{array}{l}\text { ETHION, } \\
\text { TOTAL } \\
\text { IN BOT- } \\
\text { TOM MA- } \\
\text { TERIAL } \\
\text { (UG/KG) }\end{array}$ & $\begin{array}{l}\text { HEPTA- } \\
\text { CHLOR, } \\
\text { TOTAL } \\
\text { IN BOT- } \\
\text { TOM MA- } \\
\text { TERIAL } \\
\text { (UG/KG) }\end{array}$ & $\begin{array}{c}\text { HEPTA- } \\
\text { CHLOR } \\
\text { EPOXIDE } \\
\text { TOT. IN } \\
\text { BOTTOM } \\
\text { MATL. } \\
\text { (UG/KG) }\end{array}$ & $\begin{array}{l}\text { LINDANE } \\
\text { TOTAL } \\
\text { IN BOT- } \\
\text { TOM MA- } \\
\text { TERIAL } \\
\text { (UG/KG) }\end{array}$ & $\begin{array}{c}\text { MALA- } \\
\text { THION, } \\
\text { TOTAL } \\
\text { IN BOT- } \\
\text { TOM MA- } \\
\text { TERIAL } \\
\text { (UG/KG) }\end{array}$ & $\begin{array}{c}\text { METH- } \\
\text { OXY- } \\
\text { CHLOR, } \\
\text { TOT. IN } \\
\text { BOTTOM } \\
\text { MATL. } \\
\text { (UG/KG) }\end{array}$ & $\begin{array}{c}\text { METHYL } \\
\text { PARA- } \\
\text { THION, } \\
\text { TOT. IN } \\
\text { BOTTOM } \\
\text { MATL. } \\
\text { (UG/KG) }\end{array}$ & $\begin{array}{c}\text { METHYL } \\
\text { TRI- } \\
\text { THION, } \\
\text { TOT. IN } \\
\text { BOTTOM } \\
\text { MATL. } \\
\text { (UG/KG) }\end{array}$ & $\begin{array}{l}\text { PARA- } \\
\text { THION, } \\
\text { TOTAL } \\
\text { IN BOT- } \\
\text { TOM MA- } \\
\text { TERIAL } \\
\text { (UG/KG) }\end{array}$ & $\begin{array}{l}\text { TOXA- } \\
\text { PHENE, } \\
\text { TOTAL } \\
\text { IN BOT- } \\
\text { TOM MA- } \\
\text { TERIAL } \\
\text { (UG/KG) }\end{array}$ & $\begin{array}{l}\text { TRI- } \\
\text { THION, } \\
\text { TOTAL } \\
\text { IN BOT- } \\
\text { TOM MA- } \\
\text { TERIAL } \\
\text { (UG/KG) }\end{array}$ \\
\hline $\begin{array}{c}\text { AUG }, 1 \\
15 . .1\end{array}$ & 1980 & .0 & .0 & .0 & .0 & .0 & .0 & .0 & .0 & .00 & .0 \\
\hline DATE & TIME & $\begin{array}{l}\text { STREAM- } \\
\text { FLOW, } \\
\text { INSTAN- } \\
\text { TANEOUS } \\
\text { (CFS) }\end{array}$ & $\begin{array}{l}\text { SEDI- } \\
\text { MENT, } \\
\text { SUS- } \\
\text { PENDED } \\
\text { (MG/L) }\end{array}$ & $\begin{array}{l}\text { SED. } \\
\text { SUSP. } \\
\text { FALL } \\
\text { DIAM. } \\
\% \text { FINER } \\
\text { THAN } \\
.002 \text { MM }\end{array}$ & $\begin{array}{l}\text { SED. } \\
\text { SUSP. } \\
\text { FALL } \\
\text { DIAM. } \\
\% \text { FINER } \\
\text { THAN } \\
.004 \text { MM }\end{array}$ & $\begin{array}{c}\text { SED. } \\
\text { SUSP. } \\
\text { FALL } \\
\text { DIAM. } \\
\% \text { FINER } \\
\text { THAN } \\
.008 \text { MM }\end{array}$ & $\begin{array}{l}\text { SED. } \\
\text { SUSP. } \\
\text { FALL } \\
\text { DIAM. } \\
\% \text { FINER } \\
\text { THAN } \\
.016 \text { MM }\end{array}$ & $\begin{array}{l}\text { SED. } \\
\text { SUSP. } \\
\text { FALL } \\
\text { DIAM. } \\
\% \text { FINER } \\
\text { THAN } \\
.031 \text { MM }\end{array}$ & $\begin{array}{l}\text { SED. } \\
\text { SUSP. } \\
\text { SIEVE } \\
\text { DIAM. } \\
\% \text { FINER } \\
\text { THAN } \\
.062 \text { MM }\end{array}$ & $\begin{array}{c}\text { SED. } \\
\text { SUSP. } \\
\text { SIEVE } \\
\text { DIAM. } \\
\% \text { FINER } \\
\text { THAN } \\
.125 \text { MM }\end{array}$ & $\begin{array}{l}\text { SED. } \\
\text { SUSP. } \\
\text { SIEVE } \\
\text { DIAM. } \\
\% \text { FINER } \\
\text { THAN } \\
.250 \mathrm{MM}\end{array}$ \\
\hline $\begin{array}{c}\text { JUN } \\
05 . .1\end{array}$ & ${ }_{1325}^{1980_{1325}}$ & 60 & 2470 & 52 & 71 & 86 & 96 & 98 & 100 & -- & $=-$ \\
\hline JUL $22 \ldots$ & 1300 & 37 & 115 & 59 & 80 & 90 & 96 & 98 & 98 & 99 & 100 \\
\hline
\end{tabular}


Table 13. Water and bed-material analyses from Onion River near Sheboygan Falls, 1979 and 1980 water years

\begin{tabular}{|c|c|c|c|c|c|c|c|c|}
\hline DATE & TIME & $\begin{array}{l}\text { STREAM- } \\
\text { FLOW, } \\
\text { INSTAN- } \\
\text { TANEOUS } \\
\text { (CFS) }\end{array}$ & $\begin{array}{c}\text { ALKA- } \\
\text { LINITY } \\
\text { FIELD } \\
\text { (MG/L } \\
\text { AS } \\
\text { CACO3) }\end{array}$ & $\begin{array}{l}\text { CHLO- } \\
\text { RIDE, } \\
\text { DIS - } \\
\text { SOLVED } \\
\text { (MG/L } \\
\text { AS CL) }\end{array}$ & $\begin{array}{c}\text { SOLIDS, } \\
\text { RESIDUE } \\
\text { AT } 180 \\
\text { DEG . C } \\
\text { DIS - } \\
\text { SOLVED } \\
\text { (MG/L) }\end{array}$ & $\begin{array}{c}\text { SOLIDS, } \\
\text { DIS- } \\
\text { SOLVED } \\
\text { (TONS } \\
\text { PER } \\
\text { AC-FT) }\end{array}$ & $\begin{array}{c}\text { SOLIDS, } \\
\text { DIS - } \\
\text { SOLVED } \\
\text { (TONS } \\
\text { PER } \\
\text { DAY) }\end{array}$ & $\begin{array}{c}\text { NITRO- } \\
\text { GEN } \\
\text { NITRATE } \\
\text { TOTAL } \\
\text { (MG/L } \\
\text { AS N) }\end{array}$ \\
\hline $\begin{array}{c}\text { DEC } \\
06 \ldots\end{array}$ & 8 & 34 & 360 & 48 & 534 & .73 & 49 & 2.40 \\
\hline $\begin{array}{l}\text { FEB } \\
28 \ldots\end{array}$ & 1145 & 32 & 320 & 31 & 446 & .61 & 39 & 1.28 \\
\hline $\begin{array}{r}\text { MAR } \\
20 \ldots \\
20 \ldots \\
23 \ldots \\
23 \ldots \\
24 \ldots \\
24 \ldots \\
30 \ldots \\
30 \ldots \\
31 \ldots \\
31 \ldots \\
31 \ldots \\
31 \ldots\end{array}$ & $\begin{array}{l}1030 \\
1200 \\
0845 \\
1045 \\
0430 \\
0700 \\
0045 \\
0300 \\
0830 \\
1100 \\
2100 \\
2330\end{array}$ & $\begin{array}{r}360 \\
360 \\
1660 \\
1780 \\
2200 \\
2010 \\
442 \\
498 \\
1850 \\
1940 \\
1710 \\
1630\end{array}$ & $\begin{array}{r}-- \\
123 \\
82 \\
-- \\
213 \\
-- \\
-- \\
139 \\
77 \\
-- \\
80 \\
--\end{array}$ & $\begin{array}{ll} & -- \\
21 & \\
16 & \\
32 & - \\
& -- \\
21 & - \\
11 & \\
11 & - \\
11 & --\end{array}$ & $\begin{array}{r}-- \\
233 \\
182 \\
-- \\
353 \\
-- \\
-- \\
251 \\
147 \\
-- \\
147 \\
--\end{array}$ & $\begin{array}{l}-32 \\
.32 \\
.25 \\
- \\
.48 \\
-. \\
- \\
.34 \\
.20 \\
-- \\
.20 \\
-.-\end{array}$ & $\begin{array}{c}226 \\
816 \\
2100^{--} \\
337^{--} \\
734 \\
679\end{array}$ & $\begin{array}{c}3.33 \\
=- \\
3.06 \\
2.79 \\
3.35 \\
=- \\
1.87 \\
2.07\end{array}$ \\
\hline $\begin{array}{l}\text { APR } \\
02 \ldots \\
02 \ldots\end{array}$ & $\begin{array}{l}2030 \\
2300\end{array}$ & $\begin{array}{l}517 \\
483\end{array}$ & $\begin{array}{r}115 \\
--\end{array}$ & 15 & $\begin{array}{r}198 \\
--\end{array}$ & $\begin{array}{r}.27 \\
--\end{array}$ & 276 & $2.78^{--}$ \\
\hline $\begin{array}{r}\text { MAY } \\
25 \ldots\end{array}$ & 0945 & 45 & 290 & 30 & 451 & .61 & 55 & 1.08 \\
\hline $\begin{array}{r}\text { JUN } \\
28 \ldots . \\
29 \ldots \\
29 \ldots \\
30 \ldots \\
30 \ldots \\
30 \ldots \\
30 \ldots\end{array}$ & $\begin{array}{l}1130 \\
1830 \\
2000 \\
0330 \\
0500 \\
1230 \\
1400\end{array}$ & $\begin{array}{r}16 \\
70 \\
74 \\
113 \\
113 \\
96 \\
92\end{array}$ & $\begin{array}{r}280 \\
-\overline{-} \\
220 \\
-- \\
209 \\
-\overline{-} \\
209\end{array}$ & $\begin{array}{l}25 \\
25 \\
25 \\
25 \\
31\end{array}$ & $\begin{array}{r}380 \\
-- \\
376 \\
-- \\
418 \\
-- \\
438\end{array}$ & $\begin{array}{l}.52 \\
-\overline{-} \\
.51 \\
-\overline{-} \\
.57 \\
-\overline{60}\end{array}$ & ${ }^{16} 5^{--}-$ & $\begin{array}{c}.080 \\
3.54 \\
6.37 \\
7.32 \\
--\end{array}$ \\
\hline $\begin{array}{l}\text { JUL } \\
01 \ldots \\
01 \ldots\end{array}$ & $\begin{array}{l}1230 \\
1400\end{array}$ & $\begin{array}{l}71 \\
71\end{array}$ & 253 & $25^{--}$ & $41 \overline{1}$ & $.5 \bar{~}$ & $79^{--}$ & $\begin{array}{r}4.40 \\
-.\end{array}$ \\
\hline $\begin{array}{r}\text { AUG } \\
08 \ldots \\
10 \ldots \\
10 \ldots \\
10 \ldots \\
11 \ldots\end{array}$ & $\begin{array}{l}1430 \\
0345 \\
0645 \\
2315 \\
0045\end{array}$ & $\begin{array}{r}20 \\
69 \\
97 \\
314 \\
320\end{array}$ & $\begin{array}{r}240 \\
187 \\
-- \\
-- \\
143\end{array}$ & $\begin{array}{l}29 \\
23 \\
\\
17\end{array}$ & $\begin{array}{r}376 \\
312 \\
-- \\
274\end{array}$ & $\begin{array}{r}.51 \\
.42 \\
-- \\
.- \\
.37\end{array}$ & $\begin{array}{c}20 \\
58 \\
237^{--}\end{array}$ & $\begin{array}{r}.400 \\
2.42 \\
3.75 \\
--\end{array}$ \\
\hline
\end{tabular}


Table 13. Water and bed-material analyses from Onion River near Sheboygan Falls, 1979 and 1980 water years-Continued

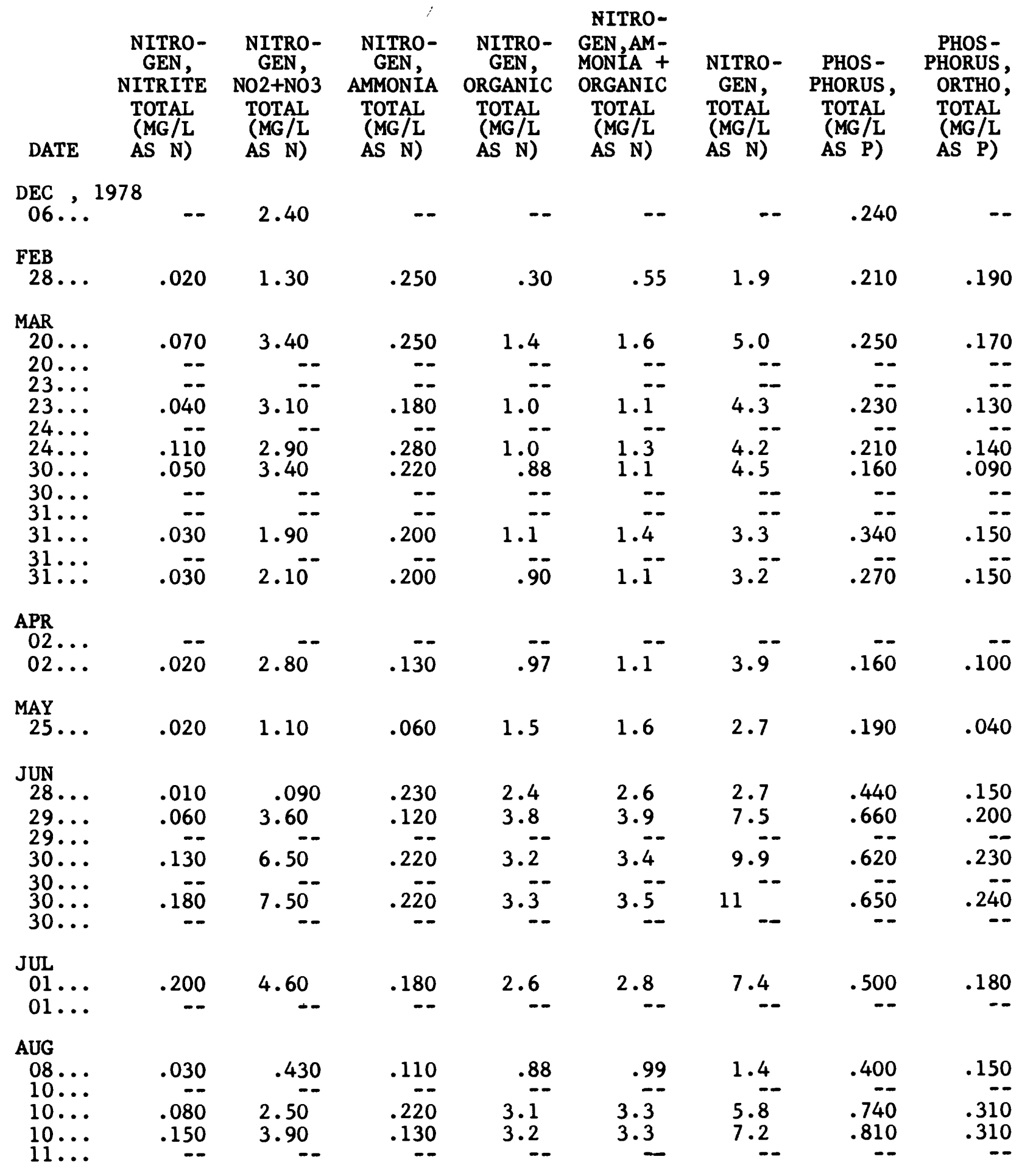


Table 13. Water and bed-material analyses from Onion River near Sheboygan Falls, 1979 and 1980 water years--Continued

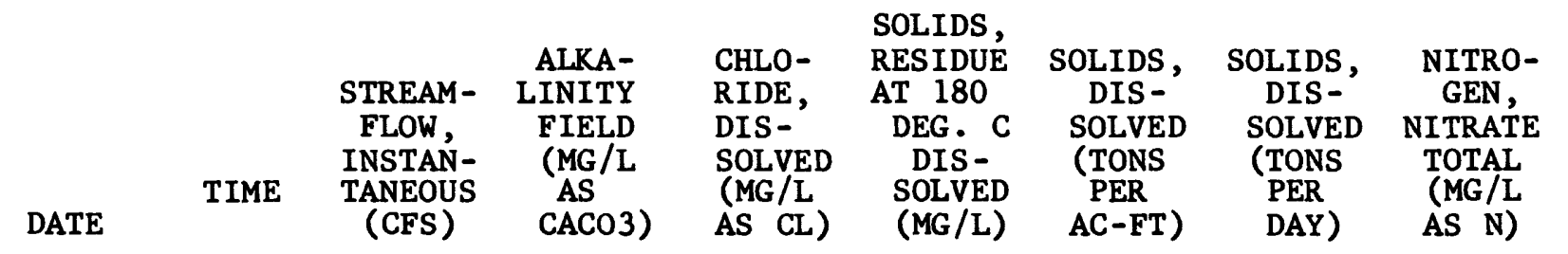

\begin{tabular}{|c|c|c|c|c|c|c|c|c|c|}
\hline \multicolumn{10}{|c|}{ AUG , 1979} \\
\hline $11 \ldots$ & 1400 & 305 & -- & & -- & $-\overline{-}$ & -- & -- & 3.43 \\
\hline $11 \ldots$ & 1830 & 284 & 187 & 21 & & 345 & .47 & 265 & $50^{--}$ \\
\hline $12 \ldots$ & 0930 & 200 & 187 & 21 & & 337 & .46 & 182 & - \\
\hline $20 \ldots$ & 1830 & 63 & 308 & 35 & & 469 & .64 & 80 & - \\
\hline $20 \ldots$ & 2000 & 70 & -- & & -- & -- & -- & & 1.79 \\
\hline $21 \ldots$ & 0630 & 137 & 209 & 36 & & 463 & .63 & 171 & \\
\hline $22 \ldots$ & 0030 & 116 & -- & & -- & -- & -- & - & 2.99 \\
\hline $22 \ldots$ & 1830 & 86 & - & & -- & $=-$ & $=-$ & - & 2.30 \\
\hline 22 & 2000 & 85 & 308 & 34 & & 473 & .64 & 109 & -- \\
\hline $25 \ldots$ & 1515 & 135 & 264 & 29 & & 439 & .60 & 160 & - \\
\hline 25 & 1645 & 129 & - & & -- & - & -- & -- & 2.83 \\
\hline 29 & 0815 & 62 & -0 & & -- & & & -- & 1.76 \\
\hline 29 & 0945 & 61 & 319 & 34 & & 476 & .65 & 78 & -- \\
\hline
\end{tabular}

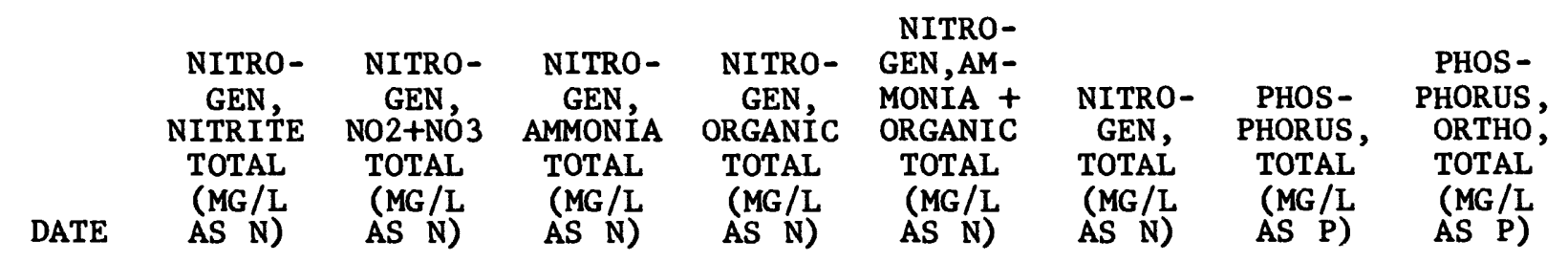

\begin{tabular}{|c|c|c|c|c|c|c|c|c|}
\hline \multicolumn{9}{|c|}{$\begin{array}{l}\text { AUG }, 1979 \\
11 . . .\end{array}$} \\
\hline & - & -. & -- & -. & - & -. & -- & -- \\
\hline $12 \ldots$ & .100 & 2.60 & .180 & 2.2 & 2.4 & 5.0 & .510 & .240 \\
\hline $12 \ldots$ & -- & & -- & -- & -- & -- & -- & -- \\
\hline $20 \ldots$ & .010 & 1.80 & .130 & 2.4 & 2.5 & 4.3 & .620 & .200 \\
\hline $21 \ldots$ & -- & -- & -- & $=-$ & $=$ & $a^{--}$ & $\overline{0}$ & \\
\hline $\begin{array}{l}22 \ldots \\
22 \ldots\end{array}$ & $\begin{array}{r}.010 \\
<.010\end{array}$ & $\begin{array}{l}3.00 \\
2.30\end{array}$ & $\begin{array}{l}.130 \\
.100\end{array}$ & $\begin{array}{l}2.5 \\
2.4\end{array}$ & $\begin{array}{l}2.6 \\
2.5\end{array}$ & $\begin{array}{l}5.6 \\
4.8\end{array}$ & $\begin{array}{l}.480 \\
.470\end{array}$ & $\begin{array}{l}.180 \\
.150\end{array}$ \\
\hline $22 \ldots$ & -- & -- & -- & -- & -- & -- & -- & -- \\
\hline 5. & -- & -- & -- & - & $=-$ & - & -- & \\
\hline 25. & $\begin{array}{l}.070 \\
.040\end{array}$ & $\begin{array}{l}2.90 \\
1.80\end{array}$ & $\begin{array}{l}.090 \\
.080\end{array}$ & $\begin{array}{l}2.4 \\
2.0\end{array}$ & $\begin{array}{l}2.5 \\
2.1\end{array}$ & $\begin{array}{l}5.4 \\
3.9\end{array}$ & $\begin{array}{r}.500 \\
.420\end{array}$ & $\begin{array}{l}.240 \\
.150\end{array}$ \\
\hline 29. & -- & -- & -- & -- & -- & -- & - & -- \\
\hline
\end{tabular}


Table 13. Water and bed-material analyses from Onion River near Sheboygan Falls, 1979 and 1980 water years-Continued

\begin{tabular}{|c|c|c|c|c|c|c|c|c|}
\hline DATE & TIME & $\begin{array}{l}\text { STREAM- } \\
\text { FLOW, } \\
\text { INSTAN'- } \\
\text { TANEOUS } \\
\text { (CFS) }\end{array}$ & $\begin{array}{c}\text { PH } \\
\text { (STAND- } \\
\text { ARD } \\
\text { UNITS) }\end{array}$ & $\begin{array}{c}\text { OXYGEN, } \\
\text { DIS- } \\
\text { SOLVED } \\
\text { (MG/L) }\end{array}$ & $\begin{array}{c}\text { OXYGEN, } \\
\text { DIS- } \\
\text { SOLVED } \\
\text { (PER- } \\
\text { CENT } \\
\text { SATUR- } \\
\text { ATION) }\end{array}$ & $\begin{array}{l}\text { COLI- } \\
\text { FORM, } \\
\text { FECAL, } \\
0.7 \\
\text { UM-MF } \\
\text { (COLS. / } \\
100 \mathrm{ML} \text { ) }\end{array}$ & $\begin{array}{l}\text { STREP- } \\
\text { TOCOCCI } \\
\text { FECAL, } \\
\text { KF AGAR } \\
\text { (COLS. } \\
\text { PER } \\
100 \text { ML) }\end{array}$ & $\begin{array}{l}\text { OXYGEN } \\
\text { DEMAND, } \\
\text { BIO- } \\
\text { CHEM- } \\
\text { ICAL, } \\
5 \text { DAY } \\
\text { (MG/L) }\end{array}$ \\
\hline $\begin{array}{c}\text { DEC }, \\
05 \ldots \\
06 \ldots \\
06 \ldots\end{array}$ & $\begin{array}{l}1735 \\
1030 \\
1915\end{array}$ & $\begin{array}{l}36 \\
35 \\
34\end{array}$ & $\begin{array}{r}7.9 \\
-.- \\
--\end{array}$ & 12.0 & $\begin{array}{l}-- \\
--\end{array}$ & $\overline{70}$ & $\overline{62}$ & $4 . \overline{-}$ \\
\hline $\begin{array}{r}\text { JAN }, \\
16 \ldots \\
17 \ldots \\
17 \ldots\end{array}$ & $\begin{array}{l}9 \\
0945 \\
1105 \\
1720\end{array}$ & $\begin{array}{l}11 \\
13 \\
13\end{array}$ & $\begin{array}{l}7.9 \\
7.9 \\
--.\end{array}$ & 3.0 & $\begin{array}{l}-- \\
--\end{array}$ & $\overline{--}$ & $-\overline{-}$ & $\begin{array}{l}-- \\
--\end{array}$ \\
\hline $\begin{array}{l}\text { FEB } \\
26 \ldots \\
28 \ldots\end{array}$ & $\begin{array}{l}0930 \\
1145\end{array}$ & $\begin{array}{l}29 \\
32\end{array}$ & $7 . \overline{4}$ & $\begin{array}{l}4.0 \\
4.0\end{array}$ & $\overline{29}$ & $1 \overline{--}$ & 240 & $2 . \overline{2}$ \\
\hline $\begin{array}{r}\text { MAR } \\
23 \ldots\end{array}$ & 1330 & 1750 & -- & -- & -- & 120 & 3100 & 3.4 \\
\hline $\begin{array}{l}\text { APR } \\
10 \ldots\end{array}$ & -- & 153 & 8.1 & 12.0 & -- & -- & -- & -- \\
\hline $\begin{array}{l}\text { MAY } \\
25 \ldots \\
25 \ldots\end{array}$ & $\begin{array}{l}0930 \\
1510\end{array}$ & $\begin{array}{l}46 \\
42\end{array}$ & -- & 11.0 & -- & 59 & -- & $\begin{array}{r}5.4 \\
-.\end{array}$ \\
\hline $\begin{array}{l}\text { JUN } \\
28 \ldots \\
28 \ldots\end{array}$ & $\begin{array}{l}1130 \\
1630\end{array}$ & $\begin{array}{l}16 \\
16\end{array}$ & $\begin{array}{c}8.6 \\
---\end{array}$ & $\begin{array}{r}9.2 \\
--\end{array}$ & $\begin{array}{r}107 \\
--\end{array}$ & 250 & $\overline{84}$ & $\begin{array}{r}4.1 \\
-.-\end{array}$ \\
\hline $\begin{array}{l}\text { AUG } \\
08 \ldots \\
08 \ldots\end{array}$ & $\begin{array}{l}1430 \\
1815\end{array}$ & $\begin{array}{l}20 \\
19\end{array}$ & $\begin{array}{r}8.0 \\
--\end{array}$ & $\overline{--}$ & -- & $1 \overline{80}$ & 200 & -- \\
\hline $\begin{array}{l}\text { SEP } \\
14 \ldots \\
14 \ldots \\
14 \ldots\end{array}$ & $\begin{array}{l}0900 \\
1500 \\
1600\end{array}$ & $\begin{array}{l}18 \\
18 \\
18\end{array}$ & $\begin{array}{c}8.8 \\
-- \\
--\end{array}$ & 10.3 & $\begin{array}{l}-- \\
-- \\
--\end{array}$ & K870 & 200 & $\begin{array}{l}-- \\
8 . \overline{1}\end{array}$ \\
\hline
\end{tabular}


Table 13. Water and bed-material analyses from Onion River near Sheboygan Falls, 1979 and 1980 water years--Continued

\begin{tabular}{|c|c|c|c|c|c|c|c|c|c|c|}
\hline DATE & TIME & $\begin{array}{l}\text { STREAM- } \\
\text { FLOW, } \\
\text { INSTAN- } \\
\text { TANEOUS } \\
\text { (CFS) }\end{array}$ & $\begin{array}{c}\text { ALKA- } \\
\text { LINITY } \\
\text { FIELD } \\
\text { (MG/L } \\
\text { AS } \\
\text { CACO3) }\end{array}$ & $\begin{array}{l}\text { CHLO- } \\
\text { RIDE, } \\
\text { DIS - } \\
\text { SOLVED } \\
\text { (MG/L } \\
\text { AS CL) }\end{array}$ & $\begin{array}{l}\text { SOLIDS, } \\
\text { RESIDUE } \\
\text { AT } 180 \\
\text { DEG. C } \\
\text { DIS- } \\
\text { SOLVED } \\
\text { (MG/L) }\end{array}$ & $\begin{array}{c}\text { SOLIDS, } \\
\text { DIS- } \\
\text { SOLVED } \\
\text { (TONS } \\
\text { PER } \\
\text { AC-FT) }\end{array}$ & $\begin{array}{c}\text { SOLID } \\
\text { DIS } \\
\text { SOLV } \\
\text { (TON } \\
\text { PEF } \\
\text { DAY }\end{array}$ & & $\begin{array}{c}\text { NITRO- } \\
\text { GEN, } \\
\text { NITRATE } \\
\text { TOTAL } \\
\text { (MG/L } \\
\text { AS N) }\end{array}$ & $\begin{array}{l}\text { NITRO- } \\
\text { GEN, } \\
\text { NITRITE } \\
\text { TOTAL } \\
\text { (MG/L } \\
\text { AS N) }\end{array}$ \\
\hline $\begin{array}{l}\text { NOV }, \\
16 . . .\end{array}$ & ${ }^{1979} 0755$ & 21 & 330 & 76 & 520 & .71 & 29 & & 1.28 & .020 \\
\hline $\begin{array}{l}\text { DEC } \\
14 \ldots\end{array}$ & 1000 & 3.9 & 320 & 30 & 421 & .57 & 4. & & 1.20 & .000 \\
\hline $\begin{array}{c}\text { JAN } \\
16 \ldots \\
17 \ldots \\
17 \ldots \\
17 \ldots \\
18 \ldots \\
18 \ldots \\
19 \ldots \\
19 \ldots \\
24 \ldots\end{array}$ & $\begin{array}{r}1980 \\
2345 \\
0115 \\
1000 \\
1430 \\
0230 \\
0700 \\
1730 \\
1900 \\
1315\end{array}$ & $\begin{array}{r}\text { E150 } \\
\text { E140 } \\
\text { E150 } \\
\text { E190 } \\
\text { E170 } \\
\text { E160 } \\
\text { E150 } \\
\text { E120 } \\
\text { E50 }\end{array}$ & $\begin{array}{r}-- \\
155 \\
-- \\
139 \\
141 \\
-- \\
-- \\
175 \\
302\end{array}$ & $\begin{array}{ll}40 & -- \\
23 & - \\
35 & \\
& -- \\
27 & - \\
41 & \end{array}$ & $\begin{array}{r}322 \\
-- \\
261 \\
289 \\
-- \\
-- \\
305 \\
487\end{array}$ & $\begin{array}{l}.-- \\
.44 \\
-\overline{.36} \\
.39 \\
-- \\
.- \\
.41 \\
.66\end{array}$ & & $\begin{array}{l}-- \\
-- \\
-- \\
-- \\
-- \\
--\end{array}$ & $\begin{array}{r}3.13 \\
.460 \\
=- \\
4.21 \\
4.03 \\
2.28\end{array}$ & $\begin{array}{r}.070 \\
.010 \\
.- \\
- \\
.090 \\
.070 \\
.020\end{array}$ \\
\hline $\begin{array}{c}\text { MAR } \\
18 \ldots \\
18 \ldots \\
19 \ldots \\
19 \ldots \\
19 \ldots \\
20 \ldots \\
20 \ldots \\
21 \ldots \\
27 \ldots\end{array}$ & $\begin{array}{l}1230 \\
1400 \\
0200 \\
1230 \\
1400 \\
0330 \\
2300 \\
0030 \\
1100\end{array}$ & $\begin{array}{r}\text { E62 } \\
\text { E58 } \\
\text { E241 } \\
\text { E273 } \\
\text { E249 } \\
\text { E221 } \\
\text { E147 } \\
\text { E123 } \\
90\end{array}$ & $\begin{array}{r}13 \overline{-} \\
\overline{110} \\
-- \\
110 \\
121 \\
\overline{--}\end{array}$ & $\begin{array}{l}35 \\
28 \\
28 \\
26 \\
26 \\
27 \\
27\end{array}$ & $\begin{array}{r}278 \\
-- \\
238 \\
--\overline{8} \\
245 \\
248 \\
\overline{348}\end{array}$ & $\begin{array}{l}.- \\
.38 \\
. \overline{-} \\
.32 \\
. \overline{ } \\
.33 \\
.34 \\
. \overline{47}\end{array}$ & 85 & $\begin{array}{l}-- \\
-- \\
-- \\
-- \\
--\end{array}$ & $\begin{array}{c}2.02 \\
1.64 \\
1.64 \\
=- \\
1.74 \\
1.58\end{array}$ & $\begin{array}{r}.080 \\
-- \\
.060 \\
-- \\
.060 \\
-- \\
-0 \\
.060 \\
.020\end{array}$ \\
\hline $\begin{array}{c}\text { APR } \\
05 \ldots \\
05 \ldots \\
05 \ldots \\
08 \ldots \\
08 \ldots \\
09 \ldots \\
09 \ldots \\
09 \ldots \\
09 \ldots \\
10 \ldots \\
10 \ldots\end{array}$ & $\begin{array}{l}0215 \\
0815 \\
1715 \\
1115 \\
1245 \\
0215 \\
0345 \\
1845 \\
2015 \\
0815 \\
1000\end{array}$ & $\begin{array}{l}257 \\
270 \\
236 \\
238 \\
268 \\
387 \\
394 \\
455 \\
455 \\
429 \\
422\end{array}$ & $\begin{array}{r}-- \\
-- \\
-- \\
209 \\
\overline{176} \\
\overline{187} \\
\overline{--} \\
18 \overline{7}\end{array}$ & $\begin{array}{ll} & =- \\
39 & -- \\
32 & -- \\
33^{--} & - \\
32^{--} & \end{array}$ & $\begin{array}{r}-- \\
\overline{--} \\
477 \\
\overline{-} \\
429 \\
\overline{47} \\
\overline{--} \\
4 \overline{3}\end{array}$ & $\begin{array}{l}-- \\
-- \\
-\overline{6} \\
.65 \\
-\overline{58} \\
. \overline{61} \\
-- \\
. \overline{59}\end{array}$ & $\begin{array}{l}307 \\
448 \\
549 \\
493\end{array}$ & -- & $\begin{array}{c}3.21 \\
7.20 \\
7.49 \\
6.81 \\
6.52 \\
7.21 \\
6.52 \\
--\end{array}$ & $\begin{array}{r}.090 \\
.100 \\
.110 \\
-- \\
.090 \\
.- \\
.080 \\
-- \\
.090 \\
.080 \\
--\end{array}$ \\
\hline $\begin{array}{c}\text { MAY } \\
08 \ldots .\end{array}$ & 0915 & 27 & 290 & 32 & -- & -- & & - & .010 & .020 \\
\hline
\end{tabular}


Table 13. Water and bed-material analyses from Onion River near Sheboygan Falls, 1979 and 1980 water years-Continued

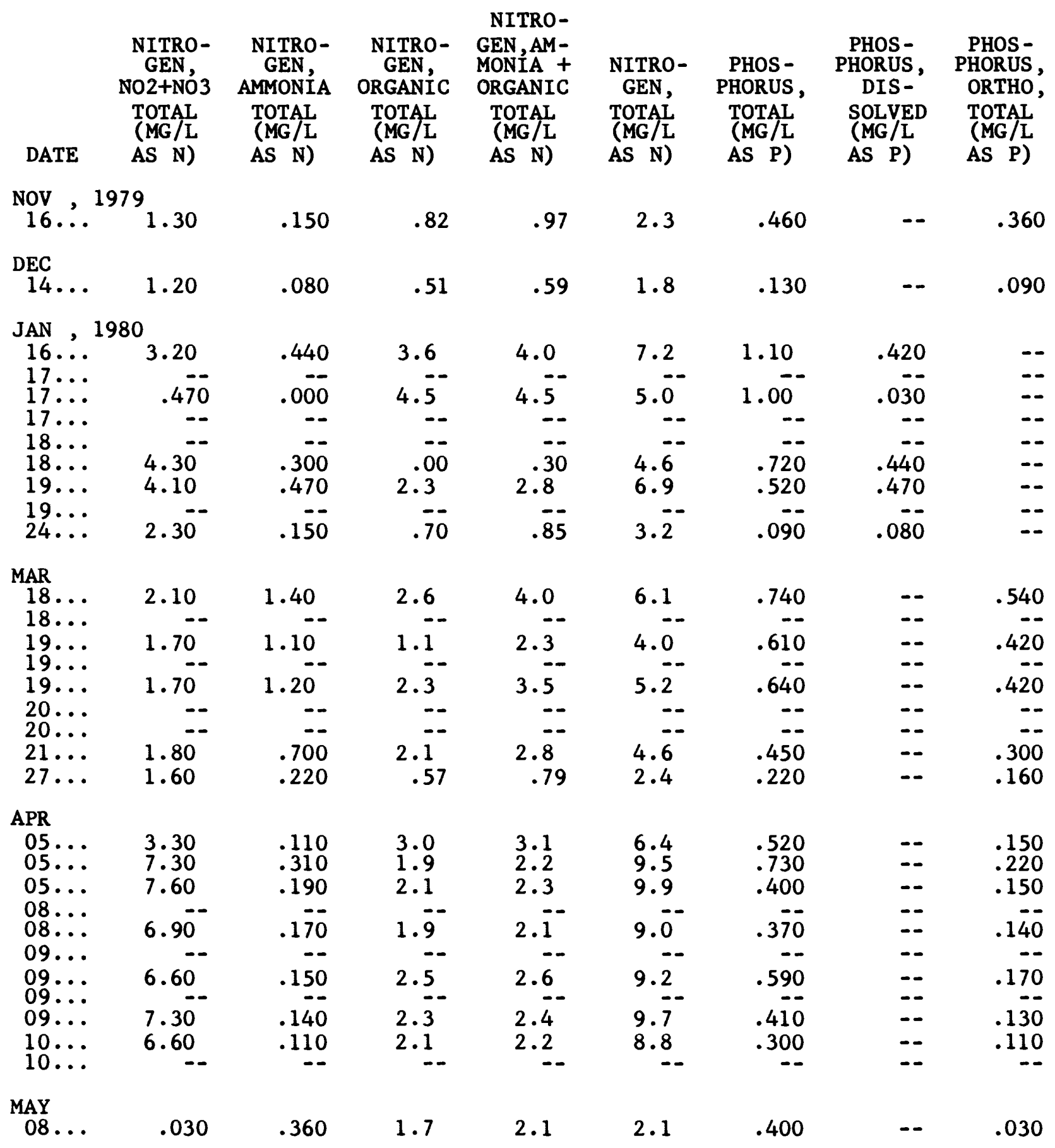


Table 13. Water and bed-material analyses from Onion River near Sheboygan Falls, 1979 and 1980 water years-Continued

\begin{tabular}{|c|c|c|c|c|c|c|c|c|c|}
\hline DATE & TIME & $\begin{array}{l}\text { STREAM- } \\
\text { FLOW, } \\
\text { INSTAN- } \\
\text { TANEOUS } \\
\text { (CFS) }\end{array}$ & $\begin{array}{c}\text { ALKA- } \\
\text { LINITY } \\
\text { FIELD } \\
\text { (MG/L } \\
\text { AS } \\
\text { CACO3) }\end{array}$ & $\begin{array}{l}\text { CHLO- } \\
\text { RIDE, } \\
\text { DIS- } \\
\text { SOLVED } \\
\text { (MG/L } \\
\text { AS CL) }\end{array}$ & $\begin{array}{c}\text { SOLIDS, } \\
\text { RESIDUE } \\
\text { AT } 180 \\
\text { DEG. C } \\
\text { DIS- } \\
\text { SOLVED } \\
\text { (MG/L) }\end{array}$ & $\begin{array}{c}\text { SOLIDS, } \\
\text { DIS - } \\
\text { SOLVED } \\
\text { (TONS } \\
\text { PER } \\
\text { AC-FT) }\end{array}$ & $\begin{array}{c}\text { SOLIDS, } \\
\text { DIS - } \\
\text { SOLVED } \\
\text { (TONS } \\
\text { PER } \\
\text { DAY) }\end{array}$ & $\begin{array}{c}\text { NITRO- } \\
\text { GEN, } \\
\text { NITRATE } \\
\text { TOTAL } \\
\text { (MG/L } \\
\text { AS N) }\end{array}$ & $\begin{array}{c}\text { NITRO- } \\
\text { GEN, } \\
\text { NITRITE } \\
\text { TOTAL } \\
\text { (MG/L } \\
\text { AS N) }\end{array}$ \\
\hline \multicolumn{10}{|c|}{ JUN , 1980} \\
\hline $05 \ldots$ & 2345 & 54 & -- & \multirow[b]{2}{*}{29} & -- & -- & -- & 1.93 & .070 \\
\hline $06 \ldots$ & 004 & 60 & 275 & & -- & -- & -- & -- & - \\
\hline $06 \ldots$ & 0945 & 127 & - & \multirow{2}{*}{$=$} & - & -- & - & 8.64 & .260 \\
\hline $06 \ldots$ & 1245 & 141 & -- & & -- & -- & -- & 2.39 & .110 \\
\hline $06 \ldots$ & 2145 & 188 & 209 & \multirow{2}{*}{31} & - & -- & -- & - & - \\
\hline $06 \ldots$ & 2315 & 108 & - & & -- & -- & -- & 8.38 & .220 \\
\hline $07 \ldots$ & 0515 & 167 & -- & $22^{--}$ & -- & -- & -- & .740 & .220 \\
\hline $08 \ldots$ & 0515 & 211 & 242 & 33 & - & -- & -- & -- & -- \\
\hline $08 \ldots$ & 0645 & 211 & - & \multirow{2}{*}{-} & -- & -- & -- & 9.31 & .190 \\
\hline $09 \ldots$ & 0345 & 180 & -- & & -- & -- & -- & 4.57 & .130 \\
\hline $09 \ldots$ & 1545 & 200 & -- & \multirow{2}{*}{$22^{--}$} & -- & -- & -- & 2.79 & .110 \\
\hline $10 \ldots$ & 111 & 15 & 220 & & -- & -- & -- & & -- \\
\hline $10 \ldots$ & 1245 & 136 & - & $21^{--}$ & -- & -- & -- & 9.52 & .280 \\
\hline $12 \ldots$ & 123 & 50 & 210 & 21 & -- & -- & - & 1.20 & .100 \\
\hline $17 \ldots$ & 1030 & 30 & 66 & 9.0 & 280 & .38 & 23 & .700 & .030 \\
\hline 20. & 0930 & 73 & -- & & -- & $=$ & & 4.49 & .110 \\
\hline & 1100 & 86 & 198 & 24 & 350 & .48 & 81 & & \\
\hline $21 \ldots$ & 0030 & 249 & - & -- & - & - & -- & 6.58 & .220 \\
\hline $21 \ldots$ & 0500 & 254 & 165 & 23 & 333 & .45 & 228 & & - \\
\hline $21 \ldots$ & 1230 & 21 & - & \multirow{2}{*}{$6^{--}$} & - & -- & & 5.31 & .190 \\
\hline $21 \ldots$ & 1700 & 18 & 187 & & 361 & .49 & 175 & & - \\
\hline $22 \ldots$ & 1100 & 112 & - & \multirow{2}{*}{$26^{--}$} & -- & -- & & 4.16 & .140 \\
\hline $22 \ldots$ & 12 & 108 & 242 & & 405 & .55 & 118 & & \\
\hline $08 \ldots$ & 1015 & 8. & -- & \multirow[t]{2}{*}{--} & -- & & -- & 2.09 & .110 \\
\hline 08. & 1145 & 104 & 198 & & 298 & .41 & 84 & & \\
\hline $09 \ldots$ & 0700 & 313 & $=$ & -- & - & -- & -- & 3.07 & .130 \\
\hline $09 \ldots$ & 0830 & 320 & 165 & \multirow{2}{*}{$\begin{array}{l}21 \\
29\end{array}$} & 301 & .41 & 260 & -- & -- \\
\hline & 0230 & 241 & 220 & & 394 & .54 & 256 & & $\overline{170}$ \\
\hline & 1000 & 188 & -- & -- & -- & -- & 10 & 3.93 & .170 \\
\hline $11 \ldots$ & 1445 & $\begin{array}{l}105 \\
102\end{array}$ & 264 & 31 & $\begin{array}{r}429 \\
--\end{array}$ & .58 & 122 & $0 a^{--}$ & $\overline{40}$ \\
\hline & 090 & 41 & 320 & 48 & 511 & .70 & 57 & .410 & .030 \\
\hline & 1015 & 79 & - & -- & - & -- & -- & .590 & .100 \\
\hline
\end{tabular}


Table 13. Water and bed-material analyses from Onion River near Sheboygan Falls, 1979 and 1980 water years-Continued

\begin{tabular}{|c|c|c|c|c|c|c|c|c|}
\hline DATE & $\begin{array}{c}\text { NITRO- } \\
\text { GEN, } \\
\text { NO2+NO3 } \\
\text { TOTAL } \\
\text { (MG/L } \\
\text { AS N) }\end{array}$ & $\begin{array}{l}\text { NITRO- } \\
\text { GEN, } \\
\text { AMMONIA } \\
\text { TOTAL } \\
\text { (MG/L } \\
\text { AS N) }\end{array}$ & $\begin{array}{l}\text { NITRO- } \\
\text { GEN, } \\
\text { ORGANIC } \\
\text { TOTAL } \\
\text { (MG/L } \\
\text { AS N) }\end{array}$ & $\begin{array}{l}\text { NITRO- } \\
\text { GEN,AM- } \\
\text { MONIA + } \\
\text { ORGANIC } \\
\text { TOTAL } \\
\text { (MG/L } \\
\text { AS N) }\end{array}$ & $\begin{array}{l}\text { NITRO- } \\
\text { GEN, } \\
\text { TOTAL } \\
\text { (MG/L } \\
\text { AS N) }\end{array}$ & $\begin{array}{c}\text { PHOS- } \\
\text { PHORUS, } \\
\text { TOTAL } \\
\text { (MG/L } \\
\text { AS P) }\end{array}$ & $\begin{array}{c}\text { PHOS - } \\
\text { PHORUS, } \\
\text { DIS- } \\
\text { SOLVED } \\
\text { (MG/L } \\
\text { AS P) }\end{array}$ & $\begin{array}{c}\text { PHOS- } \\
\text { PHORUS, } \\
\text { ORTHO, } \\
\text { TOTAL } \\
\text { (MG/L } \\
\text { AS P) }\end{array}$ \\
\hline \multicolumn{9}{|c|}{ JUN , 1980,00} \\
\hline $\begin{array}{l}05 \ldots \\
06 \ldots\end{array}$ & 2.00 & .100 & 2.4 & 2.5 & 4.5 & .550 & -- & .190 \\
\hline $\begin{array}{l}06 \ldots \\
06 \ldots\end{array}$ & & $.2 \overline{0}$ & $4.2^{--}$ & $4.4^{--}$ & & 940 & -- & .280 \\
\hline $06 \ldots$ & 2.50 & .120 & 2.7 & 2.8 & 5.3 & .580 & -- & .240 \\
\hline $06 \ldots$ & -- & $=-$ & -- & -- & -- & -- & - & -- \\
\hline $\begin{array}{l}06 \ldots \\
07 \ldots\end{array}$ & $\begin{array}{r}8.60 \\
.960\end{array}$ & $\begin{array}{l}.210 \\
.210\end{array}$ & $\begin{array}{l}3.0 \\
3.1\end{array}$ & $\begin{array}{l}3.2 \\
3.3\end{array}$ & $\frac{12}{4.3}$ & $\begin{array}{l}.630 \\
.570\end{array}$ & $=-$ & $\begin{array}{l}.220 \\
.220\end{array}$ \\
\hline $08 \ldots$ & - & $\overline{-}$ & $0^{--}$ & -- & -- & -- & -- & - \\
\hline $\begin{array}{l}08 \ldots \\
09 \ldots \\
09 \ldots\end{array}$ & $\begin{array}{l}9.50 \\
4.70 \\
2.90\end{array}$ & $\begin{array}{r}180 \\
.120 \\
.130\end{array}$ & $\begin{array}{l}3.0 \\
2.8 \\
3.1\end{array}$ & $\begin{array}{l}3.2 \\
2.9 \\
3.2\end{array}$ & $\begin{array}{r}13 \\
7.6 \\
6.1\end{array}$ & $\begin{array}{l}.630 \\
.550 \\
.590\end{array}$ & $\begin{array}{l}-- \\
--\end{array}$ & $\begin{array}{r}.210 \\
.180 \\
.210\end{array}$ \\
\hline $10 \ldots$ & $=-$ & $-\overline{-}$ & $=-$ & -- & & -- & -- & $=$ \\
\hline $\begin{array}{l}10 \ldots \\
12 \ldots\end{array}$ & $\begin{array}{l}9.80 \\
1.30\end{array}$ & $\begin{array}{l}.220 \\
.160\end{array}$ & $\begin{array}{l}3.5 \\
1.4\end{array}$ & $\begin{array}{l}3.7 \\
1.6\end{array}$ & $\begin{array}{r}14 \\
2.9\end{array}$ & $\begin{array}{l}.850 \\
.270\end{array}$ & $=-$ & $\begin{array}{l}.240 \\
.110\end{array}$ \\
\hline $\begin{array}{l}\text { JUL } \\
17 . . .\end{array}$ & .730 & .020 & 2.2 & 2.2 & 2.9 & .610 & -- & 160 \\
\hline $20 \ldots$ & 4.60 & .240 & 3.4 & $3 . \overline{6}$ & 8.2 & .890 & -- & .360 \\
\hline $\begin{array}{l}20 \ldots \\
21 \ldots\end{array}$ & $680^{--}$ & $2 \pi$ & $38^{--}$ & -- & +- & $0^{--}$ & -- & $\overline{5}$ \\
\hline $21 \ldots$ & 0.00 & .220 & 3.8 & $\begin{array}{l}4.0 \\
-.-\end{array}$ & 11 & 1.10 & - & .350 \\
\hline $21 \ldots$ & 5.50 & .170 & 2.7 & 2.9 & 8.4 & .620 & -- & .250 \\
\hline $21 \ldots$ & & -- & 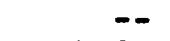 & & & 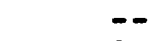 & & \\
\hline 22 . & 4.30 & . 140 & 2.8 & 2.9 & 7.2 & .580 & -- & .200 \\
\hline AUG 22 & -- & -- & -- & -- & -- & -- & -- & -- \\
\hline $08 \ldots$ & 2.20 & .320 & 2.9 & 3.2 & 5.4 & .910 & -- & .550 \\
\hline $08 \ldots$ & & $2=-$ & $\overline{7}$ & $0^{--}$ & - & 730 & -- & $\overline{7}$ \\
\hline 09 & 3.20 & .230 & 2.7 & 2.9 & 6.1 & .730 & -- & .470 \\
\hline 09 & -- & -- & -- & -- & -- & -- & -- & -- \\
\hline $\begin{array}{l}10 \\
10\end{array}$ & $4.10^{--}$ & .150 & $2.3^{--}$ & $2 . \overline{4}$ & $6 . \overline{5}$ & .550 & $=-$ & $3 \overline{40}$ \\
\hline $11 \ldots$ & -- & -1 & 2.0 & 2.4 & 0.2 & - & - & . $04+40$ \\
\hline $11 \ldots$ & 3.20 & .150 & 2.2 & 2.3 & 5.5 & .530 & -- & .290 \\
\hline $\begin{array}{l}15 . \\
29 .\end{array}$ & $\begin{array}{l}.440 \\
.690\end{array}$ & $\begin{array}{l}.090 \\
.030\end{array}$ & $\begin{array}{l}1.7 \\
1.8\end{array}$ & $\begin{array}{l}1.8 \\
1.8\end{array}$ & $\begin{array}{l}2.2 \\
2.5\end{array}$ & .390 & $=-$ & $\begin{array}{l}.180 \\
.290\end{array}$ \\
\hline
\end{tabular}


Table 13. Water and bed-material analyses from Onion River near Sheboygan Falls, 1979 and 1980 water years--Continued

\begin{tabular}{|c|c|c|c|c|c|c|c|c|c|}
\hline DATE & TIME & $\begin{array}{c}\text { STREAM- } \\
\text { FLOW, } \\
\text { INSTAN- } \\
\text { TANEOUS } \\
\text { (CFS) }\end{array}$ & $\begin{array}{l}\text { ALKA- } \\
\text { LINITY } \\
\text { FIELD } \\
\text { (MG/L } \\
\text { AS } \\
\text { CACO3) }\end{array}$ & $\begin{array}{l}\text { CHLO- } \\
\text { RIDE, } \\
\text { DIS- } \\
\text { SOLVED } \\
\text { (MG/L } \\
\text { AS CL) }\end{array}$ & $\begin{array}{l}\text { SOLIDS, } \\
\text { RESIDUE } \\
\text { AT } 180 \\
\text { DEG. C } \\
\text { DIS - } \\
\text { SOLVED } \\
(M G / L)\end{array}$ & $\begin{array}{c}\text { SOLIDS, } \\
\text { DIS- } \\
\text { SOLVED } \\
\text { (TONS } \\
\text { PER } \\
\text { AC-FT) }\end{array}$ & $\begin{array}{c}\text { SOLIDS, } \\
\text { DIS- } \\
\text { SOLVED } \\
\text { (TONS } \\
\text { PER } \\
\text { DAY) }\end{array}$ & $\begin{array}{l}\text { NITRO- } \\
\text { GEN, } \\
\text { NITRATE } \\
\text { TOTAL } \\
\text { (MG/L } \\
\text { AS N) }\end{array}$ & $\begin{array}{c}\text { NITRO- } \\
\text { GEN, } \\
\text { NITRITE } \\
\text { TOTAL } \\
\text { (MG/L } \\
\text { AS N) }\end{array}$ \\
\hline \multicolumn{10}{|c|}{ SEP , 1980} \\
\hline $17 \ldots$ & 1015 & 89 & -- & -- & -- & -- & -- & 2.80 & .100 \\
\hline $17 \ldots$ & 1315 & 101 & -- & -- & -- & -- & -- & 2 & .090 \\
\hline $18 \ldots$ & 0415 & 74 & -- & -- & -- & -- & -- & 2.8 & .110 \\
\hline $20 \ldots$ & 1915 & 93 & -- & -- & -- & -- & -- & 2.21 & .090 \\
\hline & 0115 & 127 & -- & -- & -- & $=-$ & -- & .900 & 1.00 \\
\hline 21 . & 0245 & 130 & 308 & 57 & 520 & .71 & 183 & & \\
\hline 22 . & 0215 & 98 & -- & -- & -- & -- & -- & 3.29 & .210 \\
\hline 22 . & 0645 & 246 & 220 & 34 & 386 & .53 & 256 & & $=-$ \\
\hline $22 \ldots$ & 0815 & 329 & -- & -- & -- &.- & -- & 2.98 & .120 \\
\hline 22. & 2345 & 555 & $=-$ & -- & $=-$ & $=-$ & $=$ & 2.91 & .090 \\
\hline 23 & 2215 & 764 & 143 & 15 & 255 & .35 & 526 & & -- \\
\hline 23. & 2345 & 769 & -- & -- & - & -- & -- & 2.69 & .110 \\
\hline 24. & 1445 & 67 & -- & $=-$ & -- & -- & -- & 2.77 & .130 \\
\hline & 1330 & 139 & -- & -- & -- & -- & -- & & .110 \\
\hline 28 . & 0730 & 117 & -- & -- & -- & -- & -- & 4.00 & .100 \\
\hline 0 & 1630 & 0 & -- & -- & -- & -- & -- & 3.18 & .120 \\
\hline & 1330 & 7 & 297 & 39 & 503 & .68 & 102 & -- & $=-$ \\
\hline & 1500 & 7 & -- & -- & -- & -- & -- & 3.06 & .140 \\
\hline
\end{tabular}

\begin{tabular}{|c|c|c|c|c|c|c|c|c|}
\hline DATE & $\begin{array}{c}\text { NITRO- } \\
\text { GEN, } \\
\text { NO2+NO3 } \\
\text { TOTAL } \\
\text { (MG/L } \\
\text { AS N) }\end{array}$ & $\begin{array}{l}\text { NITRO- } \\
\text { GEN, } \\
\text { AMMONIA } \\
\text { TOTAL } \\
\text { (MG/L } \\
\text { AS N) }\end{array}$ & $\begin{array}{c}\text { NITRO- } \\
\text { GEN, } \\
\text { ORGANIC } \\
\text { TOTAL } \\
(\text { MG/L } \\
\text { AS N) }\end{array}$ & $\begin{array}{l}\text { NITRO- } \\
\text { GEN,AM- } \\
\text { MONIA + } \\
\text { ORGANIC } \\
\text { TOTAL } \\
\text { (MG/L } \\
\text { AS N) }\end{array}$ & $\begin{array}{l}\text { NITRO- } \\
\text { GEN, } \\
\text { TOTAL } \\
\text { (MG/L } \\
\text { AS N) }\end{array}$ & $\begin{array}{c}\text { PHOS - } \\
\text { PHORUS, } \\
\text { TOTAL } \\
\text { (MG/L } \\
\text { AS P) }\end{array}$ & $\begin{array}{c}\text { PHOS - } \\
\text { PHORUS } \\
\text { DIS- } \\
\text { SOLVED } \\
\text { (MG/L } \\
\text { AS P) }\end{array}$ & $\begin{array}{l}\text { PHOS- } \\
\text { PHORUS, } \\
\text { ORTHO, } \\
\text { TOTAL } \\
\text { (MG/L } \\
\text { AS P) }\end{array}$ \\
\hline \multicolumn{9}{|c|}{ SEP , 1980} \\
\hline $\begin{array}{l}17 \ldots \\
17 \ldots \\
18 \ldots \\
20 \ldots \\
21 \ldots\end{array}$ & $\begin{array}{l}2.90 \\
2.60 \\
3.00 \\
2.30 \\
1.90\end{array}$ & $\begin{array}{l}.180 \\
.190 \\
.000 \\
.070 \\
.360\end{array}$ & $\begin{array}{l}1.9 \\
1.8 \\
2.4 \\
2.4 \\
4.0\end{array}$ & $\begin{array}{l}2.1 \\
2.0 \\
2.4 \\
2.5 \\
4.4 \\
-.\end{array}$ & $\begin{array}{l}5.0 \\
4.6 \\
5.4 \\
4.8 \\
6.3 \\
-\end{array}$ & $\begin{array}{l}.630 \\
.590 \\
.510 \\
.610 \\
1.30\end{array}$ & $\begin{array}{l}-- \\
-- \\
-- \\
-- \\
--\end{array}$ & $\begin{array}{l}.340 \\
.300 \\
.180 \\
.360 \\
.900\end{array}$ \\
\hline $22 \ldots$ & 3.50 & .170 & 1.7 & 1.9 & 5.4 & .440 & -- & .230 \\
\hline $\begin{array}{l}22 \ldots \\
22 \ldots \\
22 \ldots\end{array}$ & $\begin{array}{l}3.10 \\
3.00\end{array}$ & $\begin{array}{l}.140 \\
.140\end{array}$ & $\begin{array}{l}4.1 \\
2.3\end{array}$ & $\begin{array}{l}4.2 \\
2.4\end{array}$ & $\begin{array}{l}7.3 \\
5.4\end{array}$ & $\begin{array}{c}1.10^{--} \\
.910\end{array}$ & $\begin{array}{l}-- \\
-- \\
--\end{array}$ & $\begin{array}{r}.320 \\
.470\end{array}$ \\
\hline $23 \ldots$ & $280^{--}$ & 120 & $\overline{6}^{--}$ & $\overline{7}$ & $45^{--}$ & 660 & $=$ & 40 \\
\hline $\begin{array}{l}24 \ldots \\
26 \ldots \\
28 \ldots \\
29 \ldots\end{array}$ & $\begin{array}{l}2.00 \\
2.90 \\
3.10 \\
4.10 \\
3.30\end{array}$ & $\begin{array}{l}.120 \\
.120 \\
.110 \\
.130 \\
.090\end{array}$ & $\begin{array}{l}1.0 \\
1.8 \\
1.4 \\
1.9 \\
1.9\end{array}$ & $\begin{array}{l}1.9 \\
1.9 \\
1.5 \\
2.0 \\
2.0\end{array}$ & $\begin{array}{l}4.3 \\
4.8 \\
4.6 \\
6.1 \\
5.3\end{array}$ & $\begin{array}{l}.000 \\
.590 \\
.410 \\
.410 \\
.360\end{array}$ & $\begin{array}{l}-- \\
-- \\
--\end{array}$ & $\begin{array}{l}.400 \\
.370 \\
.260 \\
.220 \\
.180\end{array}$ \\
\hline $\begin{array}{l}30 \ldots \\
30 \ldots\end{array}$ & 3.20 & .110 & $1 . \overline{7}$ & $1 . \overline{8}$ & $5 . \overline{0}$ & .370 & $=$ & 190 \\
\hline
\end{tabular}


Table 13. Water and bed-material analyses from Onion River near Sheboygan Falls, 1979 and 1980 water years--Continued

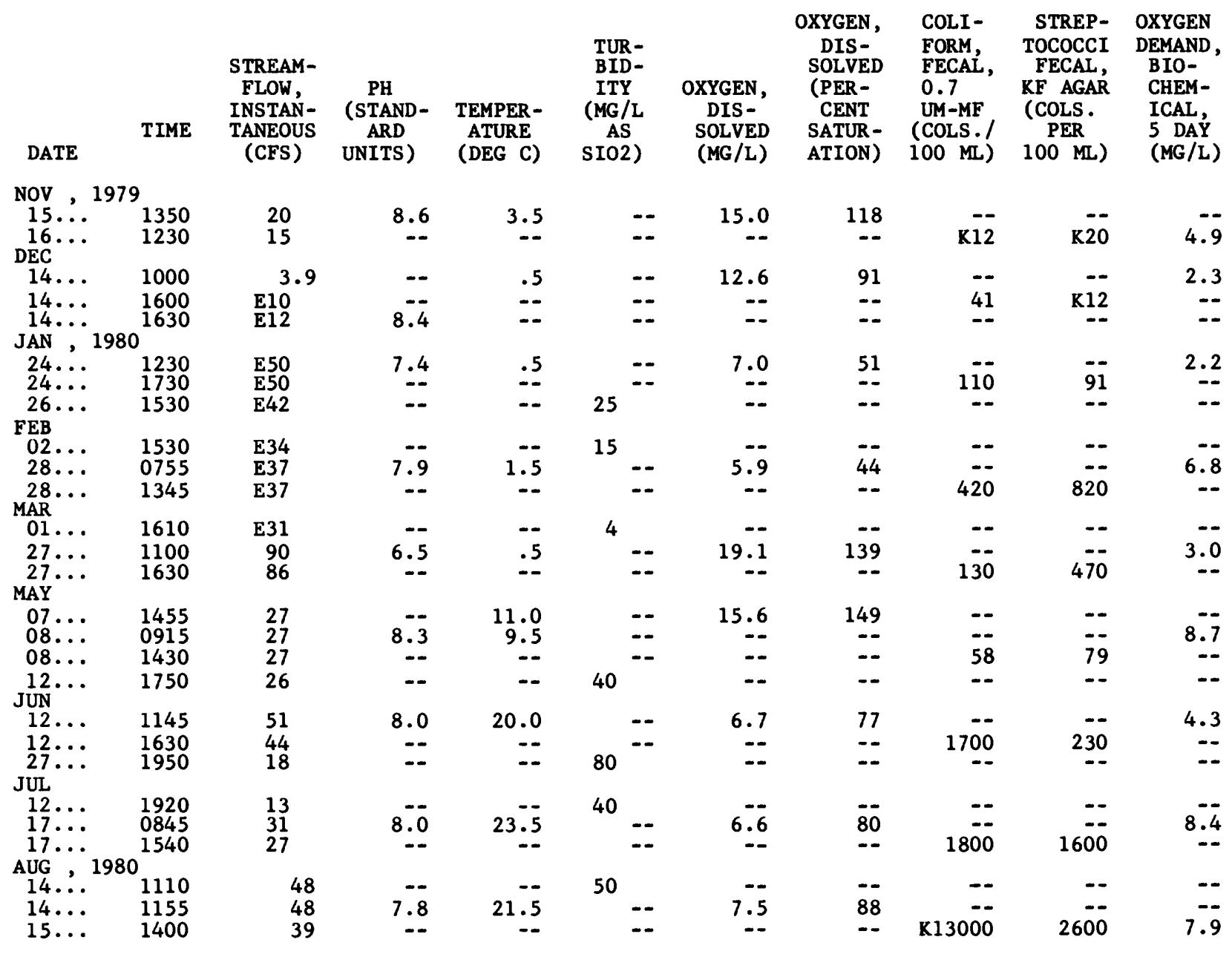


Table 13. Water and bed-material analyses from Onion River near Sheboygan Falls, 1979 and 1980 water years-Continued

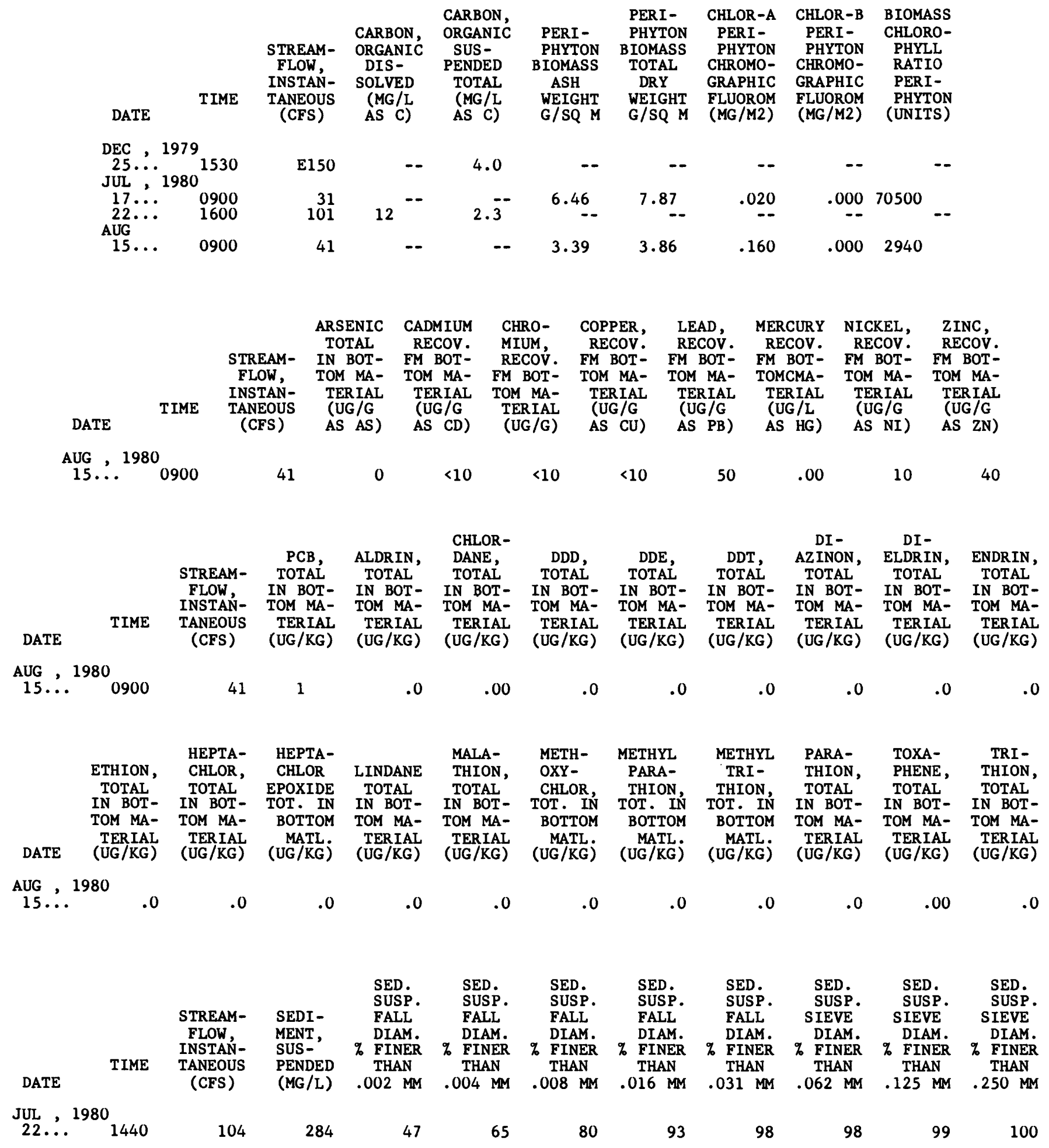


Table 14. Water temperatures for Onion River at Hingham, 1979 and 1980 water years

TEMPERATURE, WATER (DEG. C), WATER YEAR OCTOBER 1978 TO SEPTEMBER 1979

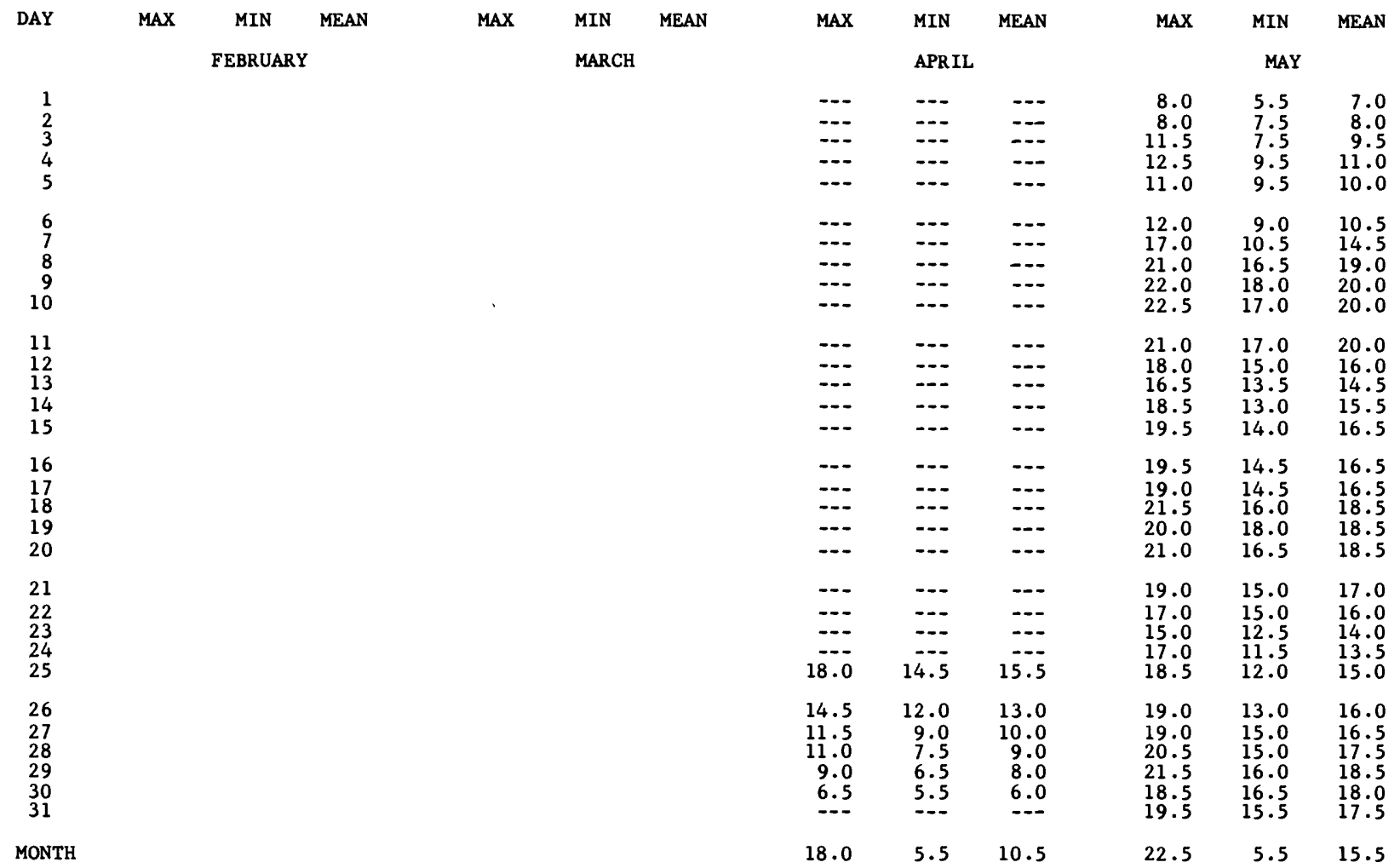

TEMPERATURE, WATER (DEG. C), WATER YEAR OCTOBER 1978 TO SEPTEMBER 1979

\begin{tabular}{|c|c|c|c|c|c|c|c|c|c|c|c|c|}
\hline DAY & $\operatorname{MAX}$ & $\begin{array}{l}\text { MIN } \\
\text { JUNE }\end{array}$ & MEAN & MAX & $\begin{array}{l}\text { MIN } \\
\text { JULY }\end{array}$ & MEAN & $\operatorname{MAX}$ & $\begin{array}{l}\text { MIN } \\
\text { AUGUS? }\end{array}$ & MEAN & $\operatorname{MAX}$ & $\begin{array}{l}\text { MIN } \\
\text { SEPTEM }\end{array}$ & MEAN \\
\hline $\begin{array}{l}1 \\
2 \\
3 \\
4 \\
5\end{array}$ & $\begin{array}{l}23.0 \\
24.0 \\
25.0 \\
22.5 \\
23.5\end{array}$ & $\begin{array}{l}18.0 \\
18.0 \\
18.5 \\
20: 0 \\
18.5\end{array}$ & $\begin{array}{l}20.0 \\
21.0 \\
21.5 \\
21.0 \\
21.0\end{array}$ & $\begin{array}{l}24.0 \\
24.5 \\
24.5 \\
24.5 \\
25.0\end{array}$ & $\begin{array}{l}20.5 \\
21.0 \\
21.5 \\
20.5 \\
19.5\end{array}$ & $\begin{array}{l}22.5 \\
22.5 \\
23.0 \\
22.5 \\
21.5\end{array}$ & $\begin{array}{l}24.0 \\
27.0 \\
29.0 \\
26.0 \\
26.5\end{array}$ & $\begin{array}{l}22.0 \\
21.0 \\
22.5 \\
24.5 \\
23.0\end{array}$ & $\begin{array}{l}23.0 \\
23.5 \\
25.5 \\
25.0 \\
24.5\end{array}$ & $\begin{array}{l}25.0 \\
25.5 \\
23.5 \\
23.0 \\
25.0\end{array}$ & $\begin{array}{l}22.5 \\
22.5 \\
21.0 \\
19.5 \\
19.5\end{array}$ & $\begin{array}{l}23.5 \\
24.0 \\
22.5 \\
21.0 \\
21.5\end{array}$ \\
\hline $\begin{array}{r}6 \\
7 \\
8 \\
9 \\
10\end{array}$ & $\begin{array}{l}21.5 \\
23.5 \\
24.0 \\
22.0 \\
21.0\end{array}$ & $\begin{array}{l}18.5 \\
19.5 \\
21.0 \\
20.5 \\
17.5\end{array}$ & $\begin{array}{l}20.0 \\
21.0 \\
22.5 \\
21.0 \\
20.0\end{array}$ & $\begin{array}{l}25.5 \\
24.5 \\
25.0 \\
26.5 \\
27.5\end{array}$ & $\begin{array}{l}20.0 \\
20.5 \\
21.0 \\
21.5 \\
23.0\end{array}$ & $\begin{array}{l}22.5 \\
22.5 \\
23.0 \\
24.0 \\
24.5\end{array}$ & $\begin{array}{l}26.0 \\
28.5 \\
26.5 \\
24.5 \\
23.0\end{array}$ & $\begin{array}{l}22.5 \\
23.5 \\
22.0 \\
23.0 \\
20.5\end{array}$ & $\begin{array}{l}24.0 \\
26.0 \\
25.5 \\
23.5 \\
21.5\end{array}$ & $\begin{array}{l}24.5 \\
21.0 \\
20.5 \\
20.5 \\
18.5\end{array}$ & $\begin{array}{l}21.0 \\
17.0 \\
15.5 \\
16.0 \\
16.5\end{array}$ & $\begin{array}{l}22.5 \\
19.0 \\
17.0 \\
18.0 \\
18.0\end{array}$ \\
\hline $\begin{array}{l}11 \\
12 \\
13 \\
14 \\
15\end{array}$ & $\begin{array}{l}21.5 \\
21.5 \\
22.5 \\
24.5 \\
27.0\end{array}$ & $\begin{array}{l}16.5 \\
18.0 \\
18.0 \\
19.5 \\
21.5\end{array}$ & $\begin{array}{l}19.0 \\
19.5 \\
20.5 \\
22.0 \\
24.5\end{array}$ & $\begin{array}{l}28.0 \\
28.5 \\
28.5 \\
30.0 \\
30.0\end{array}$ & $\begin{array}{l}23.5 \\
24.5 \\
25.5 \\
25.5 \\
25.0\end{array}$ & $\begin{array}{l}25.5 \\
26.0 \\
26.5 \\
27.5 \\
27.0\end{array}$ & $\begin{array}{l}21.5 \\
21.5 \\
20.5 \\
21.0 \\
25.5\end{array}$ & $\begin{array}{l}18.5 \\
19.0 \\
19.5 \\
17.0 \\
16.5\end{array}$ & $\begin{array}{l}20.0 \\
20.5 \\
20.0 \\
19.0 \\
19.5\end{array}$ & $\begin{array}{l}18.5 \\
22.5 \\
22.0 \\
19.0 \\
19.0\end{array}$ & $\begin{array}{l}16.5 \\
17.0 \\
19.0 \\
16.0 \\
14.0\end{array}$ & $\begin{array}{l}17.5 \\
19.5 \\
20.0 \\
17.5 \\
16.5\end{array}$ \\
\hline $\begin{array}{l}16 \\
17 \\
18 \\
19 \\
20\end{array}$ & $\begin{array}{l}27.0 \\
26.0 \\
23.5 \\
23.5 \\
25.0\end{array}$ & $\begin{array}{l}23.0 \\
21.5 \\
20.0 \\
18.5 \\
19.0\end{array}$ & $\begin{array}{l}25.0 \\
23.0 \\
21.5 \\
21.0 \\
21.5\end{array}$ & $\begin{array}{l}28.0 \\
26.5 \\
28.0 \\
27.5 \\
26.5\end{array}$ & $\begin{array}{l}23.5 \\
22.5 \\
21.0 \\
22.0 \\
22.5\end{array}$ & $\begin{array}{l}25.5 \\
24.0 \\
24.0 \\
24.5 \\
24.0\end{array}$ & $\begin{array}{l}20.5 \\
20.0 \\
21.5 \\
21.0 \\
20.5\end{array}$ & $\begin{array}{l}17.5 \\
18.0 \\
19.0 \\
19.5 \\
19.5\end{array}$ & $\begin{array}{l}19.0 \\
19.0 \\
20.0 \\
20.5 \\
20.0\end{array}$ & $\begin{array}{l}21.0 \\
21.0 \\
20.5 \\
19.0 \\
20.0\end{array}$ & $\begin{array}{l}15.0 \\
15.5 \\
16.5 \\
14.5 \\
14.5\end{array}$ & $\begin{array}{l}17.5 \\
18.0 \\
18.0 \\
16.5 \\
17.0\end{array}$ \\
\hline $\begin{array}{l}21 \\
22 \\
23 \\
24 \\
25\end{array}$ & $\begin{array}{l}25.0 \\
22.5 \\
21.5 \\
22.5 \\
23.5\end{array}$ & $\begin{array}{l}21.0 \\
19.0 \\
17.0 \\
17.0 \\
17.0\end{array}$ & $\begin{array}{l}23.0 \\
21.0 \\
19.0 \\
19.5 \\
20.0\end{array}$ & $\begin{array}{l}27.5 \\
27.5 \\
29.5 \\
27.5 \\
25.5\end{array}$ & $\begin{array}{l}22.0 \\
23.0 \\
24.0 \\
24.5 \\
24.0\end{array}$ & $\begin{array}{l}24.5 \\
25.0 \\
26.0 \\
25.5 \\
25.0\end{array}$ & $\begin{array}{l}21.5 \\
22.0 \\
23.0 \\
21.5 \\
23.0\end{array}$ & $\begin{array}{l}18.5 \\
20.5 \\
20.5 \\
20.0 \\
18.5\end{array}$ & $\begin{array}{l}20.5 \\
21.0 \\
21.5 \\
21.0 \\
21.0\end{array}$ & $\begin{array}{l}19.5 \\
18.5 \\
18.5 \\
17.0 \\
19.0\end{array}$ & $\begin{array}{l}15.5 \\
14.0 \\
14.0 \\
15.0 \\
14.5\end{array}$ & $\begin{array}{l}17.0 \\
16.0 \\
16.0 \\
16.0 \\
16.5\end{array}$ \\
\hline $\begin{array}{l}26 \\
27 \\
28 \\
29 \\
30 \\
31\end{array}$ & $\begin{array}{r}24.5 \\
24.5 \\
25.0 \\
22.5 \\
24.0 \\
\cdots\end{array}$ & $\begin{array}{l}18.0 \\
20.5 \\
20.0 \\
19.0 \\
20.0\end{array}$ & $\begin{array}{r}21.0 \\
22.0 \\
22.0 \\
21.5 \\
22.0 \\
\cdots\end{array}$ & $\begin{array}{l}27.0 \\
27.5 \\
28.0 \\
28.0 \\
27.0 \\
26.0\end{array}$ & $\begin{array}{l}23.0 \\
23.0 \\
24.0 \\
23.0 \\
23.5 \\
23.5\end{array}$ & $\begin{array}{l}24.5 \\
25.0 \\
25.5 \\
25.0 \\
25.0 \\
24.5\end{array}$ & $\begin{array}{l}21.5 \\
22.0 \\
22.0 \\
22.0 \\
24.5 \\
25.0\end{array}$ & $\begin{array}{l}19.5 \\
20.0 \\
20.5 \\
20.5 \\
21.0 \\
21.0\end{array}$ & $\begin{array}{l}20.5 \\
21.0 \\
21.0 \\
21.0 \\
22.0 \\
23.0\end{array}$ & $\begin{array}{l}20.5 \\
21.5 \\
21.5 \\
19.5 \\
21.0\end{array}$ & $\begin{array}{l}15.0 \\
16.5 \\
17.0 \\
18.0 \\
17.0\end{array}$ & $\begin{array}{l}17.5 \\
18.5 \\
19.0 \\
18.5 \\
18.5\end{array}$ \\
\hline ONTH & 27.0 & 16.5 & 21.5 & 30.0 & 19.5 & 24.5 & 29.0 & 16.5 & 21.5 & 25.5 & 14.0 & 18.5 \\
\hline
\end{tabular}

$\begin{array}{llll}\text { YEAR } & 30.0 & 5.5 & 20.0\end{array}$

NOTE: NUMBER OF MISSING DAYS OF RECORD EXCEEDED $20 \%$ OF YEAR 
Table 14. Water temperatures for Onion River at Hingham, 1979 and 1980 water years-Continued

TEMPERATURE, WATER (DEG. C), WATER YEAR OCTOBER 1979 TO SEPTEMBER 1980

\begin{tabular}{|c|c|c|c|c|c|c|c|c|c|c|c|c|}
\hline \multirow[t]{2}{*}{ DAY } & $\operatorname{MAX}$ & MIN & MEAN & MAX & MIN & MEAN & $\operatorname{MAX}$ & MIN & MEAN & $\operatorname{MAX}$ & MIN & MEAN \\
\hline & \multicolumn{3}{|c|}{ OCTOBER } & \multicolumn{3}{|c|}{ NOVEMBER } & \multicolumn{3}{|c|}{ DECEMBER } & \multicolumn{3}{|c|}{ JANUARY } \\
\hline $\begin{array}{l}1 \\
2 \\
3 \\
4 \\
5\end{array}$ & $\begin{array}{l}20.0 \\
16.5 \\
15.0 \\
13.5 \\
13.0\end{array}$ & $\begin{array}{l}15.5 \\
14.5 \\
13.0 \\
11.0 \\
10.5\end{array}$ & $\begin{array}{l}17.5 \\
15.5 \\
14.0 \\
12.5 \\
11.5\end{array}$ & $\begin{array}{r}11.0 \\
8.0 \\
6.5 \\
6.0 \\
6.5\end{array}$ & $\begin{array}{l}7.5 \\
6.0 \\
4.0 \\
3.5 \\
5.0\end{array}$ & $\begin{array}{l}9.5 \\
7.0 \\
5.5 \\
4.5 \\
5.5\end{array}$ & $\begin{array}{l}2.0 \\
2.5 \\
2.5 \\
3.0 \\
3.5\end{array}$ & $\begin{array}{r}1.0 \\
.5 \\
1.0 \\
1.5 \\
2.5\end{array}$ & $\begin{array}{l}1.5 \\
1.5 \\
2.0 \\
2.5 \\
2.5\end{array}$ & $\begin{array}{l}2.0 \\
2.0 \\
2.0 \\
2.5 \\
2.5\end{array}$ & $\begin{array}{l}1.5 \\
1.5 \\
1.0 \\
1.0 \\
1.0\end{array}$ & $\begin{array}{l}2.0 \\
1.5 \\
1.5 \\
1.5 \\
1.5\end{array}$ \\
\hline $\begin{array}{r}6 \\
7 \\
8 \\
9 \\
10\end{array}$ & $\begin{array}{l}12.5 \\
12.0 \\
11.0 \\
10.0 \\
10.0\end{array}$ & $\begin{array}{r}10.5 \\
9.5 \\
10.0 \\
8.0 \\
7.5\end{array}$ & $\begin{array}{r}11.5 \\
10.5 \\
10.5 \\
9.0 \\
8.5\end{array}$ & $\begin{array}{l}6.0 \\
5.0 \\
4.5 \\
3.5 \\
2.5\end{array}$ & $\begin{array}{r}4.5 \\
3.0 \\
2.5 \\
1.5 \\
.0\end{array}$ & $\begin{array}{l}5.5 \\
4.0 \\
3.5 \\
2.5 \\
1.5\end{array}$ & $\begin{array}{l}3.5 \\
3.5 \\
2.5 \\
3.5 \\
4.0\end{array}$ & $\begin{array}{l}2.0 \\
1.5 \\
1.0 \\
2.0 \\
2.0\end{array}$ & $\begin{array}{l}2.5 \\
2.5 \\
2.0 \\
2.5 \\
3.0\end{array}$ & $\begin{array}{r}1.5 \\
.5 \\
.5 \\
.5 \\
1.0\end{array}$ & $\begin{array}{l}.5 \\
.5 \\
.5 \\
.5 \\
.5\end{array}$ & $\begin{array}{r}1.0 \\
.5 \\
.5 \\
.5 \\
.5\end{array}$ \\
\hline $\begin{array}{l}11 \\
12 \\
13 \\
14 \\
15\end{array}$ & $\begin{array}{l}9.5 \\
8.5 \\
7.5 \\
7.5 \\
9.5\end{array}$ & $\begin{array}{l}8.5 \\
5.5 \\
4.5 \\
4.5 \\
5.5\end{array}$ & $\begin{array}{l}9.0 \\
7.5 \\
6.0 \\
6.0 \\
7.5\end{array}$ & $\begin{array}{l}2.5 \\
4.0 \\
5.0 \\
5.5 \\
5.0\end{array}$ & $\begin{array}{r}.5 \\
2.0 \\
3.0 \\
3.0 \\
3.5\end{array}$ & $\begin{array}{l}1.5 \\
3.0 \\
3.5 \\
4.0 \\
4.5\end{array}$ & $\begin{array}{l}4.0 \\
3.0 \\
3.0 \\
3.0 \\
4.0\end{array}$ & $\begin{array}{r}2.5 \\
2.0 \\
1.0 \\
2.5 \\
2.0\end{array}$ & $\begin{array}{l}3.0 \\
2.5 \\
2.0 \\
1.5 \\
2.5\end{array}$ & $\begin{array}{r}1.0 \\
.5 \\
1.0 \\
2.5 \\
2.0\end{array}$ & $\begin{array}{r}.5 \\
.5 \\
.5 \\
.5 \\
1.5\end{array}$ & $\begin{array}{r}.5 \\
.5 \\
1.0 \\
1.5 \\
2.0\end{array}$ \\
\hline $\begin{array}{l}21 \\
22 \\
23 \\
24 \\
25\end{array}$ & $\begin{array}{r}19.5 \\
18.0 \\
15.5 \\
8.0 \\
6.5\end{array}$ & $\begin{array}{r}16.0 \\
16.5 \\
8.0 \\
6.5 \\
4.5\end{array}$ & $\begin{array}{r}18.0 \\
17.0 \\
11.5 \\
7.5 \\
5.5\end{array}$ & $\begin{array}{l}8.0 \\
7.5 \\
7.0 \\
5.5 \\
5.0\end{array}$ & $\begin{array}{l}7.5 \\
7.0 \\
5.0 \\
3.5 \\
3.5\end{array}$ & $\begin{array}{l}7.5 \\
7.5 \\
6.0 \\
4.5 \\
4.0\end{array}$ & $\begin{array}{l}2.5 \\
2.0 \\
2.0 \\
2.0 \\
1.5\end{array}$ & $\begin{array}{l}1.5 \\
2.0 \\
2.0 \\
1.5 \\
1.0\end{array}$ & $\begin{array}{l}2.0 \\
2.0 \\
2.0 \\
2.0 \\
1.5\end{array}$ & $\begin{array}{r}2.0 \\
2.0 \\
.5 \\
1.0 \\
1.5\end{array}$ & $\begin{array}{l}.5 \\
.5 \\
.5 \\
.5 \\
.5\end{array}$ & $\begin{array}{r}1.5 \\
1.0 \\
.5 \\
1.0 \\
1.0\end{array}$ \\
\hline $\begin{array}{l}26 \\
27 \\
28 \\
29 \\
30 \\
31\end{array}$ & $\begin{array}{r}7.0 \\
7.5 \\
9.5 \\
9.5 \\
10.0 \\
11.5\end{array}$ & $\begin{array}{l}3.5 \\
6.0 \\
6.0 \\
7.0 \\
7.5 \\
8.5\end{array}$ & $\begin{array}{r}5.5 \\
6.5 \\
7.5 \\
8.0 \\
8.5 \\
10.0\end{array}$ & $\begin{array}{l}4.5 \\
3.5 \\
2.5 \\
2.0 \\
1.5 \\
---\end{array}$ & $\begin{array}{r}3.5 \\
2.5 \\
.5 \\
.5 \\
.5 \\
-.-\end{array}$ & $\begin{array}{l}4.0 \\
3.0 \\
1.5 \\
1.0 \\
1.0 \\
\ldots-.\end{array}$ & $\begin{array}{l}1.0 \\
1.5 \\
2.0 \\
2.5 \\
3.0 \\
3.0\end{array}$ & $\begin{array}{r}1.0 \\
.5 \\
.5 \\
1.0 \\
1.0 \\
1.0\end{array}$ & $\begin{array}{l}1.0 \\
1.0 \\
1.5 \\
1.5 \\
1.5 \\
2.0\end{array}$ & $\begin{array}{l}1.0 \\
1.0 \\
1.0 \\
1.0 \\
1.0 \\
1.0\end{array}$ & $\begin{array}{l}.5 \\
.5 \\
.5 \\
.5 \\
.5 \\
.5\end{array}$ & $\begin{array}{l}.5 \\
.5 \\
.5 \\
.5 \\
.5 \\
.5\end{array}$ \\
\hline MONTH & 20.0 & 3.5 & 10.5 & 11.0 & .0 & 4.5 & 4.0 & .0 & 2.0 & 2.5 & .5 & 1.0 \\
\hline
\end{tabular}

TEMPERATURE, WATER (DEG. C), WATER YEAR OCTOBER 1979 TO SEPTEMBER 1980

\begin{tabular}{|c|c|c|c|c|c|c|c|c|c|c|c|c|}
\hline DAY & \multicolumn{3}{|c|}{ FEBRUARY } & \multicolumn{3}{|c|}{ MARCH } & \multicolumn{3}{|c|}{ APR IL } & \multicolumn{2}{|c|}{ MAY } & MEAN \\
\hline $\begin{array}{l}1 \\
2 \\
3 \\
4 \\
5\end{array}$ & $\begin{array}{l}1: 0 \\
1: 0 \\
1.0 \\
1: 0 \\
1: 0\end{array}$ & $\begin{array}{l}: 5 \\
.5 \\
.5 \\
: 5 \\
.5\end{array}$ & $\begin{array}{r}.5 \\
.5 \\
.5 \\
1.5\end{array}$ & $\begin{array}{r}1: 5 \\
1: 0 \\
2.0 \\
2: 5\end{array}$ & $\begin{array}{l}: 0 \\
: 0 \\
: 0 \\
: 5 \\
: 0\end{array}$ & $\begin{array}{r}.5 \\
.5 \\
.5 \\
1.0 \\
.5\end{array}$ & $\begin{array}{l}6.0 \\
7.5 \\
4.5 \\
4.5 \\
6.0\end{array}$ & $\begin{array}{l}2.0 \\
3.0 \\
2.0 \\
1.5 \\
3.5\end{array}$ & $\begin{array}{l}4: 0 \\
5: 0 \\
3.5 \\
3.0 \\
5: 0\end{array}$ & $\begin{array}{l}16.5 \\
20.0 \\
22.5 \\
22.5 \\
22.0\end{array}$ & $\begin{array}{l}10.5 \\
13.5 \\
15.0 \\
16.0 \\
16.5\end{array}$ & $\begin{array}{l}13.0 \\
16.5 \\
18.0 \\
19.0 \\
18.5\end{array}$ \\
\hline $\begin{array}{r}6 \\
7 \\
8 \\
9 \\
10\end{array}$ & $\begin{array}{l}1.0 \\
1.0 \\
1.0 \\
1.0 \\
1.0\end{array}$ & $\begin{array}{l}.5 \\
.5 \\
.5 \\
.5 \\
.5\end{array}$ & $\begin{array}{r}1.0 \\
.5 \\
1.0 \\
.5 \\
.5\end{array}$ & $\begin{array}{r}.5 \\
.5 \\
2.0 \\
1.5 \\
2.5\end{array}$ & $\begin{array}{l}.0 \\
.0 \\
.0 \\
.0 \\
.0\end{array}$ & $\begin{array}{r}.0 \\
.5 \\
.5 \\
.5 \\
1.0\end{array}$ & $\begin{array}{l}6.5 \\
6.5 \\
7.5 \\
6.0 \\
5.0\end{array}$ & $\begin{array}{l}5.5 \\
5.5 \\
5.0 \\
3.0 \\
2.5\end{array}$ & $\begin{array}{l}6.0 \\
6.0 \\
6.0 \\
4.5 \\
4.0\end{array}$ & $\begin{array}{l}19.0 \\
13.5 \\
10.5 \\
12.5 \\
12.0\end{array}$ & $\begin{array}{r}13.5 \\
9.5 \\
8.5 \\
7.5 \\
9.0\end{array}$ & $\begin{array}{r}16.0 \\
11.5 \\
9.5 \\
9.5 \\
10.0\end{array}$ \\
\hline $\begin{array}{l}11 \\
12 \\
13 \\
14 \\
15\end{array}$ & \begin{tabular}{r}
1.0 \\
.5 \\
$-\because-$ \\
\hdashline.- \\
.--
\end{tabular} & $\begin{array}{r}.5 \\
.5 \\
-.- \\
-. \\
-.\end{array}$ & \begin{tabular}{r}
.5 \\
.5 \\
.-- \\
\hdashline-- \\
.--
\end{tabular} & $\begin{array}{r}.5 \\
1.0 \\
1.0 \\
2.0 \\
2.0\end{array}$ & $\begin{array}{l}.0 \\
.0 \\
.0 \\
.0 \\
.0\end{array}$ & $\begin{array}{l}.0 \\
.5 \\
.5 \\
.5 \\
.5\end{array}$ & $\begin{array}{l}7.0 \\
6.5 \\
6.5 \\
4.5 \\
4.5\end{array}$ & $\begin{array}{r}3.5 \\
5.0 \\
4.5 \\
1.0 \\
.5\end{array}$ & $\begin{array}{l}5.5 \\
6.0 \\
5.5 \\
2.5 \\
3.0\end{array}$ & $\begin{array}{l}16.0 \\
18.0 \\
14.0 \\
13.5 \\
16.5\end{array}$ & $\begin{array}{r}9.5 \\
11.5 \\
11.0 \\
10.0 \\
10.5\end{array}$ & $\begin{array}{l}12.5 \\
14.0 \\
12.5 \\
11.5 \\
13.0\end{array}$ \\
\hline $\begin{array}{l}16 \\
17 \\
18 \\
19 \\
20\end{array}$ & $\begin{array}{l}--- \\
=-- \\
--- \\
---\end{array}$ & \begin{tabular}{l}
.-- \\
\hdashline.- \\
$-\because-$ \\
--
\end{tabular} & $\begin{array}{l}--- \\
--- \\
-- \\
---\end{array}$ & $\begin{array}{l}2.5 \\
.5 \\
2.0 \\
2.0 \\
1.5\end{array}$ & $\begin{array}{l}.5 \\
.0 \\
.0 \\
.5 \\
.5\end{array}$ & $\begin{array}{r}1.0 \\
.5 \\
.5 \\
1.0 \\
1.0\end{array}$ & $\begin{array}{r}6.5 \\
7.5 \\
12.0 \\
14.5 \\
16.5\end{array}$ & $\begin{array}{r}2.5 \\
5.5 \\
6.5 \\
10.0 \\
12.0\end{array}$ & $\begin{array}{r}5.0 \\
6.0 \\
9.0 \\
12.0 \\
14.0\end{array}$ & $\begin{array}{l}15.0 \\
13.5 \\
12.5 \\
15.5 \\
20.0\end{array}$ & $\begin{array}{l}12.0 \\
12.0 \\
12.0 \\
11.5 \\
15.0\end{array}$ & $\begin{array}{l}13.5 \\
12.5 \\
12.0 \\
14.0 \\
18.0\end{array}$ \\
\hline $\begin{array}{l}21 \\
22 \\
23 \\
24 \\
25\end{array}$ & $\begin{array}{l}=- \\
-- \\
--- \\
-- \\
--\end{array}$ & \begin{tabular}{l}
..- \\
\hdashline.- \\
$-\because-$ \\
$-\cdots$
\end{tabular} & \begin{tabular}{l}
$\because--$ \\
$\because--$ \\
\hdashline- \\
$\because-$ \\
--
\end{tabular} & $\begin{array}{r}2.5 \\
2.5 \\
.5 \\
1.0 \\
1.5\end{array}$ & $\begin{array}{l}.5 \\
.0 \\
.0 \\
.0 \\
.0\end{array}$ & $\begin{array}{r}1.0 \\
1.0 \\
.5 \\
.5 \\
.5\end{array}$ & $\begin{array}{l}17.5 \\
20.5 \\
17.5 \\
12.5 \\
10.0\end{array}$ & $\begin{array}{r}13.5 \\
14.5 \\
13.0 \\
8.0 \\
7.5\end{array}$ & $\begin{array}{r}15.5 \\
17.5 \\
15.5 \\
10.0 \\
8.5\end{array}$ & $\begin{array}{l}23.5 \\
24.5 \\
24.0 \\
25.0 \\
25.5\end{array}$ & $\begin{array}{l}17.0 \\
18.5 \\
19.0 \\
20.5 \\
20.5\end{array}$ & $\begin{array}{l}20.0 \\
21.0 \\
21.5 \\
22.5 \\
22.5\end{array}$ \\
\hline $\begin{array}{l}26 \\
27 \\
28 \\
29 \\
30 \\
31\end{array}$ & $\begin{array}{r}--- \\
1.5 \\
2.0 \\
.8 \\
--- \\
---\end{array}$ & $\begin{array}{r}--. \\
.0 \\
.0 \\
-0 \\
-.- \\
--\end{array}$ & $\begin{array}{r}--.5 \\
.5 \\
.5 \\
1.0 \\
\hdashline-.-\end{array}$ & $\begin{array}{l}3.5 \\
1.5 \\
1.5 \\
3.0 \\
3.5 \\
5.0\end{array}$ & $\begin{array}{r}.0 \\
.5 \\
.5 \\
1.0 \\
1.0 \\
1.5\end{array}$ & $\begin{array}{l}1.0 \\
1.0 \\
1.0 \\
1.5 \\
2.0 \\
3.0\end{array}$ & $\begin{array}{r}10.5 \\
11.5 \\
9.0 \\
9.5 \\
11.5 \\
-. .-\end{array}$ & $\begin{array}{l}7.5 \\
8.5 \\
8.0 \\
8.0 \\
9.0 \\
---\end{array}$ & $\begin{array}{r}8.5 \\
9.5 \\
8.5 \\
8.5 \\
10.0\end{array}$ & $\begin{array}{l}23.5 \\
24.5 \\
24.0 \\
22.5 \\
23.0 \\
23.0\end{array}$ & $\begin{array}{l}18.5 \\
17.5 \\
19.0 \\
20.0 \\
19.5 \\
18.5\end{array}$ & $\begin{array}{l}20.5 \\
20.5 \\
20.5 \\
21.0 \\
21.0 \\
20.5\end{array}$ \\
\hline MONTH & 2.0 & .0 & .5 & 5.0 & .0 & 1.0 & 20.5 & .5 & 7.5 & 25.5 & 7.5 & 16.5 \\
\hline
\end{tabular}


Table 14. Water temperatures for Onion River at Hingham, 1979 and 1980 water years-Continued

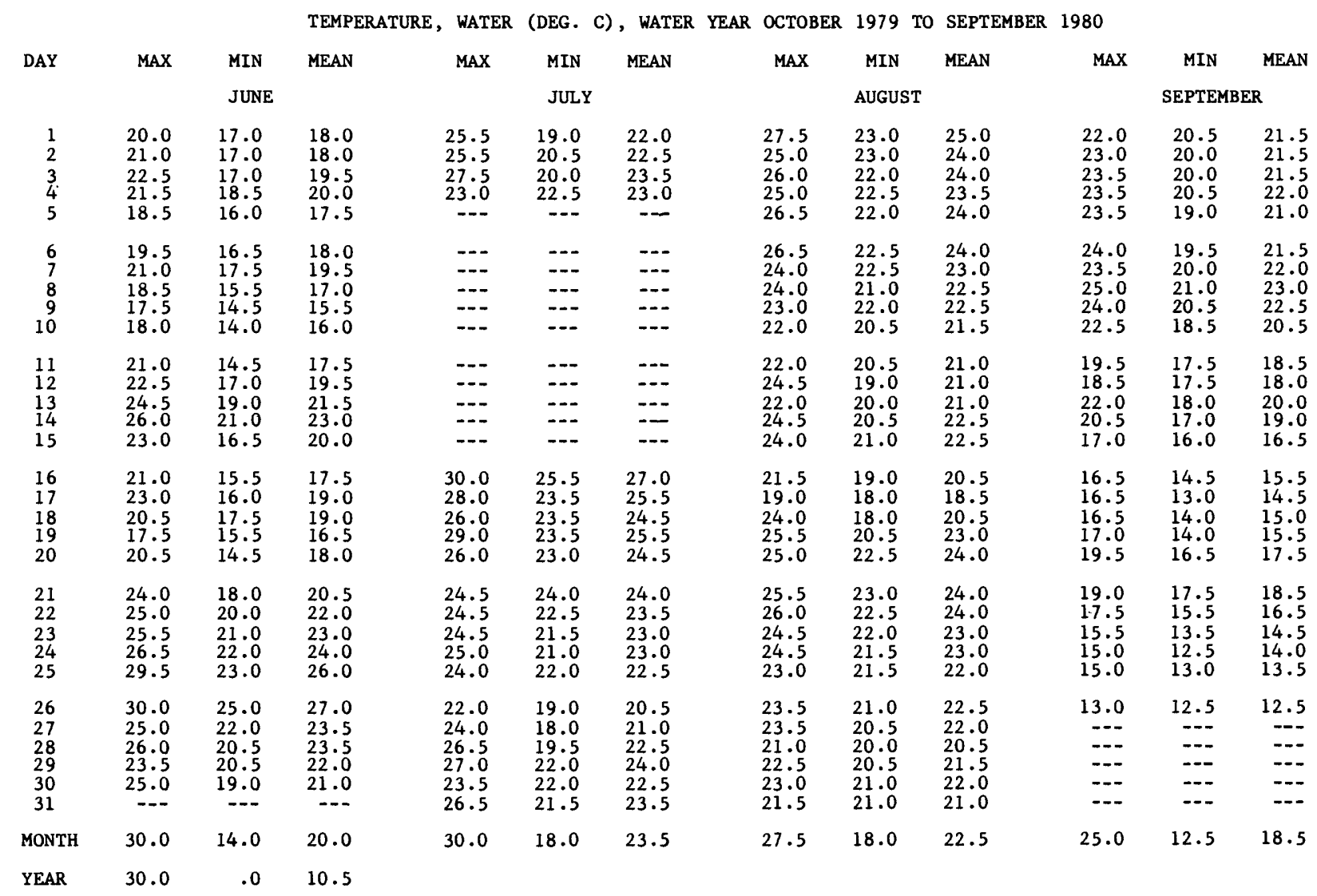


Table 15. Water temperatures for Onion River near Sheboygan Falls, 1979 and 1980 water years

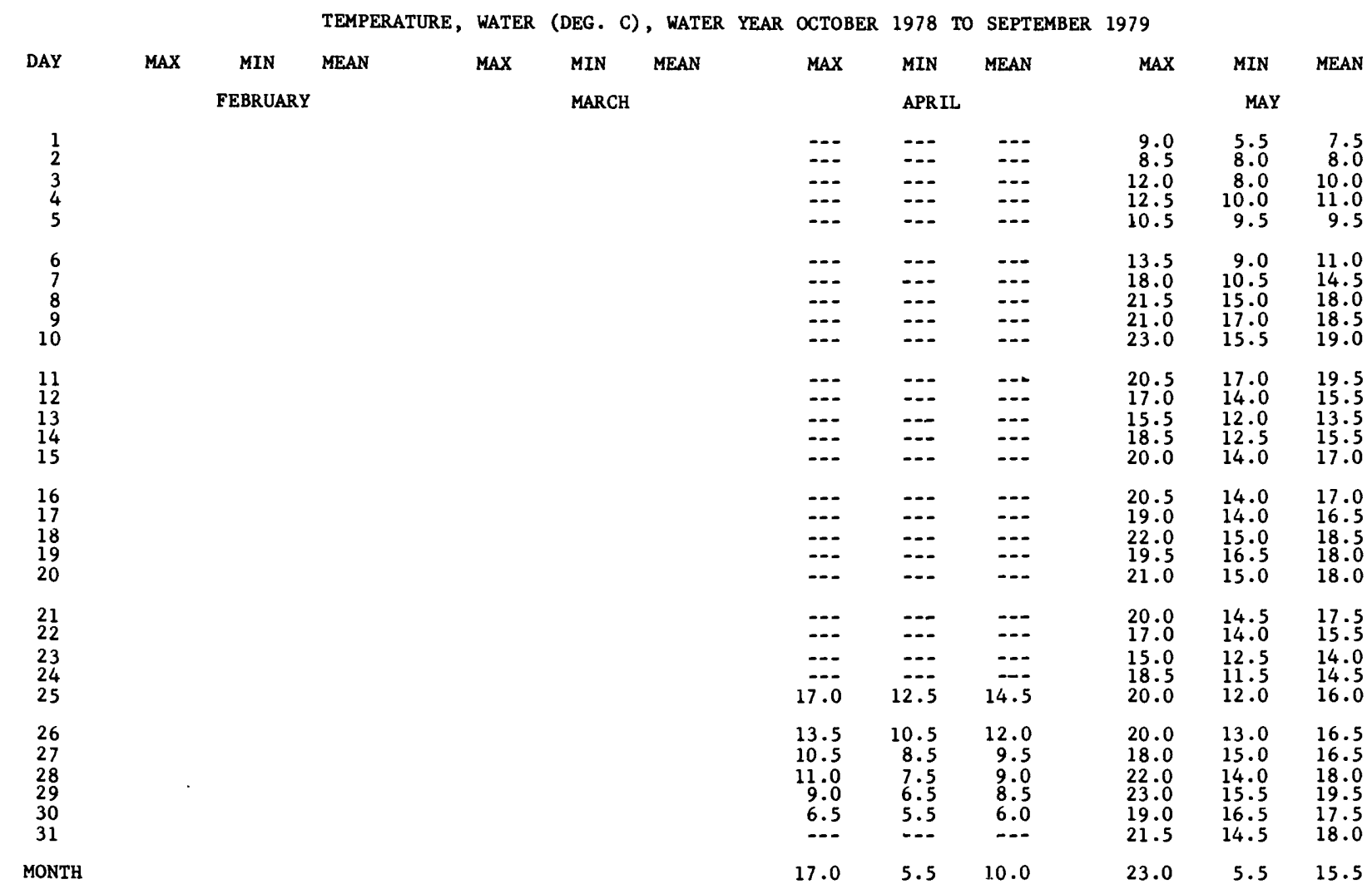

TEMPERATURE, WATER (DEG. C), WATER YEAR OCTOBER 1978 TO SEPTEMBER 1979

\begin{tabular}{|c|c|c|c|c|c|c|c|c|c|c|c|c|}
\hline \multirow[t]{2}{*}{ DAY } & $\operatorname{MAX}$ & MIN & MEAN & $\operatorname{MAX}$ & MIN & MEAN & $\operatorname{MAX}$ & MIN & MEAN & MAX & MIN & MEAN \\
\hline & \multicolumn{3}{|c|}{ JUNE } & \multicolumn{3}{|c|}{ JULY } & \multicolumn{3}{|c|}{ AUGUST } & \multicolumn{3}{|c|}{ SEPTEMBER } \\
\hline $\begin{array}{l}1 \\
2 \\
3 \\
4 \\
5\end{array}$ & $\begin{array}{l}24.0 \\
25.0 \\
25.5 \\
23.0 \\
24.5\end{array}$ & $\begin{array}{l}17.0 \\
18.0 \\
18.0 \\
19.5 \\
17.5\end{array}$ & $\begin{array}{l}20.5 \\
21.5 \\
22.0 \\
21.0 \\
21.0\end{array}$ & $\begin{array}{l}24.0 \\
25.0 \\
25.0 \\
24.0 \\
24.0\end{array}$ & $\begin{array}{l}19.0 \\
19.0 \\
20.5 \\
19.0 \\
17.0\end{array}$ & $\begin{array}{l}21.5 \\
22.0 \\
22.5 \\
21.5 \\
21.0\end{array}$ & $\begin{array}{l}23.5 \\
27.0 \\
30.0 \\
25.5 \\
27.0\end{array}$ & $\begin{array}{l}21.0 \\
19.5 \\
22.5 \\
23.5 \\
22.0\end{array}$ & $\begin{array}{l}22.0 \\
23.5 \\
26.0 \\
25.0 \\
24: 0\end{array}$ & $\begin{array}{l}25.5 \\
26.5 \\
24.0 \\
24.0 \\
25.0\end{array}$ & $\begin{array}{l}22.0 \\
22.0 \\
21.0 \\
18.0 \\
18.5\end{array}$ & $\begin{array}{l}23.5 \\
24.0 \\
22.5 \\
21.0 \\
22.0\end{array}$ \\
\hline $\begin{array}{r}6 \\
7 \\
8 \\
9 \\
10\end{array}$ & $\begin{array}{l}21.0 \\
23.0 \\
22.5 \\
21.0 \\
20.0\end{array}$ & $\begin{array}{l}17.0 \\
17.5 \\
19.5 \\
18.5 \\
16.5\end{array}$ & $\begin{array}{l}19.0 \\
20.5 \\
21.0 \\
19.5 \\
18.5\end{array}$ & $\begin{array}{l}25.5 \\
24.5 \\
25.5 \\
27.0 \\
28.5\end{array}$ & $\begin{array}{l}18.0 \\
19.0 \\
19.5 \\
20.0 \\
22.0\end{array}$ & $\begin{array}{l}22.0 \\
22.0 \\
22.5 \\
23.5 \\
24.5\end{array}$ & $\begin{array}{l}26.5 \\
29.5 \\
26.0 \\
23.5 \\
22.0\end{array}$ & $\begin{array}{l}21.0 \\
22.5 \\
23.5 \\
21.5 \\
20.5\end{array}$ & $\begin{array}{l}23.5 \\
26.0 \\
25.0 \\
22.5 \\
21.5\end{array}$ & $\begin{array}{l}25.5 \\
21.0 \\
20.0 \\
20.5 \\
18.5\end{array}$ & $\begin{array}{l}20.5 \\
17.0 \\
14.5 \\
15.0 \\
16.5\end{array}$ & $\begin{array}{l}22.5 \\
18.5 \\
17.0 \\
18.0 \\
18.0\end{array}$ \\
\hline $\begin{array}{l}11 \\
12 \\
13 \\
14 \\
15\end{array}$ & $\begin{array}{l}22.0 \\
23.0 \\
23.5 \\
25.0 \\
28.0\end{array}$ & $\begin{array}{l}15.5 \\
17.0 \\
16.5 \\
18.5 \\
20.0\end{array}$ & $\begin{array}{l}18.5 \\
20.0 \\
20.0 \\
21.5 \\
24.0\end{array}$ & $\begin{array}{l}29.5 \\
29.5 \\
29.5 \\
29.0 \\
30.0\end{array}$ & $\begin{array}{l}22.5 \\
23.0 \\
24.0 \\
24.0 \\
23.5\end{array}$ & $\begin{array}{l}25.5 \\
26.0 \\
26.0 \\
26.5 \\
26.5\end{array}$ & $\begin{array}{l}21.5 \\
22.0 \\
21.0 \\
22.0 \\
22.5\end{array}$ & $\begin{array}{l}19.5 \\
19.0 \\
19.5 \\
17.0 \\
16.0\end{array}$ & $\begin{array}{l}20.5 \\
20.5 \\
20.0 \\
19.5 \\
19.0\end{array}$ & $\begin{array}{l}18.5 \\
22.5 \\
21.0 \\
18.5 \\
19.5\end{array}$ & $\begin{array}{l}16.0 \\
15.5 \\
18.0 \\
15.0 \\
13.0\end{array}$ & $\begin{array}{l}17.0 \\
19.0 \\
19.5 \\
16.5 \\
16.0\end{array}$ \\
\hline $\begin{array}{l}16 \\
17 \\
18 \\
19 \\
20\end{array}$ & $\begin{array}{l}28.0 \\
25.5 \\
23.0 \\
24.0 \\
24.0\end{array}$ & $\begin{array}{l}23.0 \\
21.5 \\
18.0 \\
16.5 \\
17.5\end{array}$ & $\begin{array}{l}25.0 \\
23.5 \\
20.5 \\
20.5 \\
20.5\end{array}$ & $\begin{array}{l}27.5 \\
26.5 \\
26.5 \\
28.0 \\
26.0\end{array}$ & $\begin{array}{l}22.0 \\
20.5 \\
19.0 \\
20.5 \\
21.0\end{array}$ & $\begin{array}{l}24.5 \\
23.0 \\
23.0 \\
24.0 \\
23.5\end{array}$ & $\begin{array}{l}21.0 \\
19.5 \\
22.5 \\
21.5 \\
20.5\end{array}$ & $\begin{array}{l}16.0 \\
17.5 \\
18.5 \\
20.5 \\
19.5\end{array}$ & $\begin{array}{l}19.0 \\
18.5 \\
20.5 \\
21.0 \\
20.0\end{array}$ & $\begin{array}{l}21.5 \\
21.5 \\
21.5 \\
19.5 \\
20.5\end{array}$ & $\begin{array}{l}14.5 \\
15.0 \\
15.5 \\
13.0 \\
13.5\end{array}$ & $\begin{array}{l}17.5 \\
18.0 \\
18.0 \\
16.0 \\
17.0\end{array}$ \\
\hline $\begin{array}{l}21 \\
22 \\
23 \\
24 \\
25\end{array}$ & $\begin{array}{l}25.0 \\
22.0 \\
21.5 \\
23.0 \\
23.5\end{array}$ & $\begin{array}{l}19.5 \\
17.5 \\
15.0 \\
15.0 \\
15.0\end{array}$ & $\begin{array}{l}22.5 \\
20.5 \\
18.0 \\
19.0 \\
19.5\end{array}$ & $\begin{array}{l}27.5 \\
29.5 \\
30.0 \\
27.5 \\
24.5\end{array}$ & $\begin{array}{l}21.5 \\
23.0 \\
23.5 \\
24.0 \\
22.5\end{array}$ & $\begin{array}{l}24.5 \\
25.5 \\
26.5 \\
25.5 \\
24.0\end{array}$ & $\begin{array}{l}22.5 \\
22.5 \\
23.0 \\
21.5 \\
22.0\end{array}$ & $\begin{array}{l}18.5 \\
20.0 \\
20.5 \\
20.0 \\
18.5\end{array}$ & $\begin{array}{l}20.0 \\
21.0 \\
21.5 \\
20.5 \\
20.0\end{array}$ & $\begin{array}{l}19.5 \\
19.0 \\
19.0 \\
16.0 \\
19.5\end{array}$ & $\begin{array}{l}15.0 \\
12.5 \\
12.5 \\
13.5 \\
12.5\end{array}$ & $\begin{array}{l}17.0 \\
15.5 \\
15.5 \\
15.0 \\
16.0\end{array}$ \\
\hline $\begin{array}{l}26 \\
27 \\
28 \\
29 \\
30 \\
31\end{array}$ & $\begin{array}{l}25.0 \\
24.0 \\
25.0 \\
21.5 \\
23.5\end{array}$ & $\begin{array}{l}16.5 \\
19.5 \\
18.0 \\
18.5 \\
17.5\end{array}$ & $\begin{array}{l}21.0 \\
21.5 \\
21.5 \\
20.0 \\
20.5\end{array}$ & $\begin{array}{l}27.5 \\
27: 0 \\
29.0 \\
29.0 \\
28.0 \\
25.5\end{array}$ & $\begin{array}{l}21.0 \\
21: 5 \\
23.0 \\
21.5 \\
22: 5 \\
22.5\end{array}$ & $\begin{array}{l}24.0 \\
24.5 \\
25.5 \\
25.0 \\
25.0 \\
23.5\end{array}$ & $\begin{array}{l}21.0 \\
22.5 \\
23.0 \\
24.5 \\
25.5 \\
25.5\end{array}$ & $\begin{array}{l}19.0 \\
19.0 \\
20.0 \\
20.0 \\
21.0 \\
21.0\end{array}$ & $\begin{array}{l}20.0 \\
20.5 \\
21.0 \\
22.0 \\
23.0 \\
23.5\end{array}$ & $\begin{array}{l}21.0 \\
21.5 \\
21.5 \\
18.5 \\
21.0\end{array}$ & $\begin{array}{l}14.0 \\
15.5 \\
15.5 \\
16.5 \\
15.5\end{array}$ & $\begin{array}{l}17.5 \\
188.0 \\
18.5 \\
17.5 \\
18.0\end{array}$ \\
\hline MONTH & 28.0 & 15.0 & 21.0 & 30.0 & 17.0 & 24.0 & 30.0 & 16.0 & 21.5 & 26.5 & 12.5 & 18.5 \\
\hline YEAR & 30.0 & 5.5 & 19.5 & & & & & & & & & \\
\hline
\end{tabular}


Table 15. Water temperatures for Onion River near Sheboygan Falls, 1979 and 1980 water years--Continued

TEMPERATURE, WATER (DEG. C), WATER YEAR OCTOBER 1979 TO SEPTEMBER 1980

\begin{tabular}{|c|c|c|c|c|c|c|c|c|c|c|c|c|}
\hline DAY & MAX & MIN & MEAN & MAX & MIN & MEAN & MAX & MIN & MEAN & MAX & MIN & MEAN \\
\hline & \multicolumn{3}{|c|}{ OCTOBER } & \multicolumn{3}{|c|}{ NOVEMBER } & \multicolumn{3}{|c|}{ DECEMBER } & \multicolumn{3}{|c|}{ JANUARY } \\
\hline $\begin{array}{l}1 \\
2 \\
3 \\
4 \\
5\end{array}$ & $\begin{array}{l}19.0 \\
16.5 \\
14.0 \\
14.5 \\
13.5\end{array}$ & $\begin{array}{l}14.0 \\
14.0 \\
12.5 \\
10.5 \\
10.0\end{array}$ & $\begin{array}{l}16.5 \\
15.0 \\
13.0 \\
12.5 \\
11.5\end{array}$ & $\begin{array}{r}11.5 \\
8.0 \\
6.5 \\
6.0 \\
6.5\end{array}$ & $\begin{array}{l}7.0 \\
5.5 \\
4.5 \\
3.0 \\
4.5\end{array}$ & $\begin{array}{l}9.0 \\
6.5 \\
5.5 \\
4.5 \\
5.5\end{array}$ & $\begin{array}{r}.5 \\
.5 \\
.5 \\
1.0 \\
1.0\end{array}$ & $\begin{array}{r}.5 \\
.5 \\
.5 \\
.5 \\
1.0\end{array}$ & $\begin{array}{r}.5 \\
.5 \\
.5 \\
1.0 \\
1.0\end{array}$ & $\begin{array}{l}1.0 \\
1.0 \\
1.0 \\
1.0 \\
1.0\end{array}$ & $\begin{array}{l}1.0 \\
1.0 \\
1.0 \\
1.0 \\
1.0\end{array}$ & $\begin{array}{l}1.0 \\
1.0 \\
1.0 \\
1.0 \\
1.0\end{array}$ \\
\hline $\begin{array}{r}6 \\
7 \\
8 \\
9 \\
10\end{array}$ & $\begin{array}{l}13.0 \\
13.0 \\
11.5 \\
10.0 \\
11.0\end{array}$ & $\begin{array}{r}10.5 \\
9.0 \\
10.0 \\
8.0 \\
7.0\end{array}$ & $\begin{array}{r}11.5 \\
11.0 \\
11.0 \\
9.0 \\
8.5\end{array}$ & $\begin{array}{l}6.5 \\
5.5 \\
5.0 \\
3.0 \\
2.5\end{array}$ & $\begin{array}{r}4.5 \\
3.0 \\
3.0 \\
2.0 \\
.5\end{array}$ & $\begin{array}{l}6.0 \\
4.5 \\
4.0 \\
2.5 \\
1.5\end{array}$ & $\begin{array}{l}1.0 \\
1.0 \\
1.0 \\
1.0 \\
1.0\end{array}$ & $\begin{array}{l}.5 \\
1.0 \\
1.0 \\
1.0 \\
1.0\end{array}$ & $\begin{array}{l}1.0 \\
1.0 \\
1.0 \\
1.0 \\
1.0\end{array}$ & $\begin{array}{l}1.0 \\
1.0 \\
1.0 \\
1.0 \\
1.0\end{array}$ & $\begin{array}{l}1.0 \\
1.0 \\
1.0 \\
1.0 \\
1.0\end{array}$ & $\begin{array}{l}1.0 \\
1.0 \\
1.0 \\
1.0 \\
1.0\end{array}$ \\
\hline $\begin{array}{l}11 \\
12 \\
13 \\
14 \\
15\end{array}$ & $\begin{array}{r}10.0 \\
8.5 \\
8.5 \\
8.0 \\
11.0\end{array}$ & $\begin{array}{l}8.5 \\
5.5 \\
4.0 \\
4.5 \\
5.5\end{array}$ & $\begin{array}{l}9.0 \\
7.5 \\
6.0 \\
6.0 \\
8.0\end{array}$ & $\begin{array}{r}.5 \\
--- \\
--- \\
--- \\
4.5\end{array}$ & \begin{tabular}{r}
.5 \\
-- \\
\hdashline- \\
2.0
\end{tabular} & $\begin{array}{r}.5 \\
--. \\
-0 . \\
3.0\end{array}$ & $\begin{array}{l}1.5 \\
1.0 \\
1.0 \\
1.0 \\
1.0\end{array}$ & $\begin{array}{l}1.0 \\
1.0 \\
1.0 \\
1.0 \\
1.0\end{array}$ & $\begin{array}{l}1.0 \\
1.0 \\
1.0 \\
1.0 \\
1.0\end{array}$ & $\begin{array}{l}1.0 \\
1.0 \\
1.0 \\
1.0 \\
1.0\end{array}$ & $\begin{array}{l}1.0 \\
1.0 \\
1.0 \\
1.0 \\
1.0\end{array}$ & $\begin{array}{l}1.0 \\
1.0 \\
1.0 \\
1.0 \\
1.0\end{array}$ \\
\hline $\begin{array}{l}21 \\
22 \\
23 \\
24 \\
25\end{array}$ & $\begin{array}{r}19.5 \\
17.5 \\
15.0 \\
7.5 \\
6.5\end{array}$ & $\begin{array}{r}15.0 \\
15.0 \\
7.5 \\
6.5 \\
5.5\end{array}$ & $\begin{array}{r}17.5 \\
16.0 \\
10.5 \\
7.0 \\
6.0\end{array}$ & $\begin{array}{l}7.5 \\
7.5 \\
7.0 \\
5.5 \\
5.0\end{array}$ & $\begin{array}{l}7.0 \\
7.0 \\
5.0 \\
3.5 \\
3.5\end{array}$ & $\begin{array}{l}7.0 \\
7.0 \\
6.0 \\
4.5 \\
4.5\end{array}$ & $\begin{array}{l}1.0 \\
1.0 \\
1.0 \\
1.0 \\
1.0\end{array}$ & $\begin{array}{l}1.0 \\
1.0 \\
1.0 \\
1.0 \\
1.0\end{array}$ & $\begin{array}{l}1.0 \\
1.0 \\
1.0 \\
1.0 \\
1.0\end{array}$ & $\begin{array}{l}1.0 \\
1.0 \\
1.0 \\
1.0 \\
1.0\end{array}$ & $\begin{array}{l}1.0 \\
1.0 \\
1.0 \\
1.0 \\
1.0\end{array}$ & $\begin{array}{l}1.0 \\
1.0 \\
1.0 \\
1.0 \\
1.0\end{array}$ \\
\hline $\begin{array}{l}26 \\
27 \\
28 \\
29 \\
30 \\
31\end{array}$ & $\begin{array}{r}7.0 \\
7.5 \\
10.0 \\
9.5 \\
10.0 \\
12.0\end{array}$ & $\begin{array}{l}4.0 \\
6.0 \\
6.0 \\
6.0 \\
6.0 \\
8.0\end{array}$ & $\begin{array}{r}5.5 \\
6.5 \\
8.0 \\
8.0 \\
8.0 \\
10.0\end{array}$ & $\begin{array}{r}5.0 \\
4.5 \\
3.0 \\
1.0 \\
.5 \\
-.-\end{array}$ & $\begin{array}{r}4.0 \\
3.0 \\
1.0 \\
.5 \\
.5 \\
-.-\end{array}$ & $\begin{array}{r}4.5 \\
4.0 \\
2.0 \\
1.0 \\
.5 \\
--.\end{array}$ & $\begin{array}{l}1.5 \\
1.5 \\
1.5 \\
1.0 \\
1.0 \\
1.0\end{array}$ & $\begin{array}{l}1.0 \\
1.0 \\
1.0 \\
1.0 \\
1.0 \\
1.0\end{array}$ & $\begin{array}{l}1.0 \\
1.0 \\
1.0 \\
1.0 \\
1.0 \\
1.0\end{array}$ & $\begin{array}{l}1.0 \\
1.0 \\
1.0 \\
1.0 \\
1.0 \\
1.0\end{array}$ & $\begin{array}{l}1.0 \\
1.0 \\
1.0 \\
1.0 \\
1.0 \\
1.0\end{array}$ & $\begin{array}{l}1.0 \\
1.0 \\
1.0 \\
1.0 \\
1.0 \\
1.0\end{array}$ \\
\hline MONTH & 19.5 & 4.0 & 10.0 & 11.5 & .5 & 4.5 & 1.5 & .5 & 1.0 & 1.0 & 1.0 & 1.0 \\
\hline
\end{tabular}

TEMPERATURE, WATER (DEG. C), WATER YEAR OCTOBER 1979 TO SEPTEMBER 1980

\begin{tabular}{|c|c|c|c|c|c|c|c|c|c|c|c|c|}
\hline DAY & MAX & MIN & MEAN & MAX & MIN & MEAN & MAX & MIN & MEAN & $\operatorname{MAX}$ & MIN & MEAN \\
\hline \multicolumn{4}{|c|}{ FEBRUARY } & \multicolumn{3}{|c|}{ MARCH } & \multicolumn{3}{|c|}{ APRIL } & \multicolumn{3}{|c|}{ MAY } \\
\hline $\begin{array}{l}1 \\
2 \\
3 \\
4 \\
5\end{array}$ & $\begin{array}{l}1.0 \\
1.0 \\
1.0 \\
1.0 \\
1.0\end{array}$ & $\begin{array}{l}1.0 \\
1.0 \\
1.0 \\
1.0 \\
1.0\end{array}$ & $\begin{array}{l}1.0 \\
1.0 \\
1.0 \\
1.0 \\
1.0\end{array}$ & $\begin{array}{l}1.0 \\
1.0 \\
1.0 \\
1.0 \\
1.0\end{array}$ & $\begin{array}{l}1.0 \\
1.0 \\
1.0 \\
1.0 \\
1.0\end{array}$ & $\begin{array}{l}1.0 \\
1.0 \\
1.0 \\
1.0 \\
1.0\end{array}$ & $\begin{array}{r}7.5 \\
10.5 \\
7.5 \\
6.0 \\
7.0\end{array}$ & $\begin{array}{l}1.0 \\
5.0 \\
4.0 \\
1.5 \\
4.5\end{array}$ & $\begin{array}{l}4.5 \\
7.5 \\
5.5 \\
4.0 \\
6.0\end{array}$ & $\begin{array}{l}17.5 \\
20.5 \\
21.5 \\
23.0 \\
22.5\end{array}$ & $\begin{array}{l}10.5 \\
12.5 \\
13.5 \\
15.5 \\
16.5\end{array}$ & $\begin{array}{l}13.5 \\
16.0 \\
17.5 \\
19.0 \\
19.0\end{array}$ \\
\hline $\begin{array}{r}6 \\
7 \\
8 \\
9 \\
10\end{array}$ & $\begin{array}{l}1.0 \\
1.0 \\
1.0 \\
1.0 \\
1.0\end{array}$ & $\begin{array}{l}1.0 \\
1.0 \\
1.0 \\
1.0 \\
1.0\end{array}$ & $\begin{array}{l}1.0 \\
1.0 \\
1.0 \\
1.0 \\
1.0\end{array}$ & $\begin{array}{l}1.0 \\
1.0 \\
1.0 \\
1.0 \\
1.0\end{array}$ & $\begin{array}{l}1.0 \\
1.0 \\
1.0 \\
1.0 \\
1.0\end{array}$ & $\begin{array}{l}1.0 \\
1.0 \\
1.0 \\
1.0 \\
1.0\end{array}$ & $\begin{array}{l}7.5 \\
6.5 \\
6.5 \\
6.5 \\
5.0\end{array}$ & $\begin{array}{l}6.0 \\
5.5 \\
5.5 \\
4.5 \\
4.0\end{array}$ & $\begin{array}{l}6.5 \\
6.0 \\
6.0 \\
6.0 \\
4.5\end{array}$ & $\begin{array}{l}19.5 \\
14.0 \\
10.5 \\
13.5 \\
11.5\end{array}$ & $\begin{array}{r}14.0 \\
9.5 \\
8.0 \\
6.5 \\
8.5\end{array}$ & $\begin{array}{r}16.0 \\
10.5 \\
9.0 \\
10.0 \\
10.5\end{array}$ \\
\hline $\begin{array}{l}11 \\
12 \\
13 \\
14 \\
15\end{array}$ & $\begin{array}{l}1.0 \\
1.0 \\
1.0 \\
1.0 \\
1.0\end{array}$ & $\begin{array}{l}1.0 \\
1.0 \\
1.0 \\
1.0 \\
1.0\end{array}$ & $\begin{array}{l}1.0 \\
1.0 \\
1.0 \\
1.0 \\
1.0\end{array}$ & $\begin{array}{l}1.0 \\
1.0 \\
1.0 \\
1.0 \\
1.0\end{array}$ & $\begin{array}{l}1.0 \\
1.0 \\
1.0 \\
1.0 \\
1.0\end{array}$ & $\begin{array}{l}1.0 \\
1.0 \\
1.0 \\
1.0 \\
1.0\end{array}$ & $\begin{array}{l}6.5 \\
6.5 \\
6.0 \\
4.0 \\
5.0\end{array}$ & $\begin{array}{r}4.5 \\
5.0 \\
4.0 \\
1.0 \\
.5\end{array}$ & $\begin{array}{l}5.5 \\
6.0 \\
5.0 \\
2.5 \\
3.0\end{array}$ & $\begin{array}{l}17.5 \\
19.0 \\
15.0 \\
13.0 \\
17.0\end{array}$ & $\begin{array}{r}9.0 \\
11.0 \\
10.0 \\
9.0 \\
10.0\end{array}$ & $\begin{array}{l}13.0 \\
15.0 \\
12.0 \\
11.0 \\
13.5\end{array}$ \\
\hline $\begin{array}{l}21 \\
22 \\
23 \\
24 \\
25\end{array}$ & $\begin{array}{l}1.0 \\
1.0 \\
1.0 \\
1.0 \\
1.0\end{array}$ & $\begin{array}{l}1.0 \\
1.0 \\
1.0 \\
1.0 \\
1.0\end{array}$ & $\begin{array}{l}1.0 \\
1.0 \\
1.0 \\
1.0 \\
1.0\end{array}$ & $\begin{array}{l}1.0 \\
1.0 \\
1.0 \\
1.0 \\
1.0\end{array}$ & $\begin{array}{l}1.0 \\
1.0 \\
1.0 \\
1.0 \\
1.0\end{array}$ & $\begin{array}{l}1.0 \\
1.0 \\
1.0 \\
1.0 \\
1.0\end{array}$ & $\begin{array}{r}17.0 \\
20.0 \\
17.0 \\
12.5 \\
9.5\end{array}$ & $\begin{array}{r}11.5 \\
12.0 \\
12.5 \\
7.5 \\
6.5\end{array}$ & $\begin{array}{r}14.0 \\
16.0 \\
14.5 \\
9.0 \\
7.5\end{array}$ & $\begin{array}{l}24.0 \\
25.5 \\
25.0 \\
25.5 \\
25.0\end{array}$ & $\begin{array}{l}16.5 \\
18.0 \\
18.0 \\
19.5 \\
19.5\end{array}$ & $\begin{array}{l}20.5 \\
22.0 \\
22.0 \\
22.5 \\
21.5\end{array}$ \\
\hline $\begin{array}{l}26 \\
27 \\
28 \\
29 \\
30 \\
31\end{array}$ & $\begin{array}{l}1.0 \\
1.0 \\
1.0 \\
1.0 \\
-\ldots- \\
\cdots--\end{array}$ & $\begin{array}{l}1.0 \\
1.0 \\
1.0 \\
1.0 \\
---\end{array}$ & $\begin{array}{l}1.0 \\
1.0 \\
1.0 \\
1.0 \\
--- \\
---\end{array}$ & $\begin{array}{r}1.0 \\
.5 \\
.5 \\
1.0 \\
1.0 \\
4.0\end{array}$ & $\begin{array}{l}.5 \\
.5 \\
.5 \\
.5 \\
.5 \\
.5\end{array}$ & $\begin{array}{r}1.0 \\
.5 \\
.5 \\
.5 \\
.5 \\
1.5\end{array}$ & $\begin{array}{r}10.5 \\
12.0 \\
8.5 \\
10.0 \\
12.5 \\
-\end{array}$ & $\begin{array}{l}6.5 \\
8.5 \\
7.5 \\
7.5 \\
8.5 \\
-.-\end{array}$ & $\begin{array}{r}8.5 \\
9.5 \\
8.0 \\
8.5 \\
10.5 \\
-\end{array}$ & $\begin{array}{l}24.0 \\
24.5 \\
23.0 \\
22.0 \\
22.5 \\
24.5\end{array}$ & $\begin{array}{l}16.0 \\
16.5 \\
18.0 \\
18.0 \\
17.5 \\
18.0\end{array}$ & $\begin{array}{l}20.0 \\
20.5 \\
20.5 \\
19.5 \\
20.0 \\
21.0\end{array}$ \\
\hline MONTH & 1.0 & 1.0 & 1.0 & 4.0 & .5 & 1.0 & 20.0 & .5 & 7.5 & 25.5 & 6.5 & 16.5 \\
\hline
\end{tabular}


Table 15. Water temperatures for Onion River near Sheboygan Falls, 1979 and 1980 water years--Continued

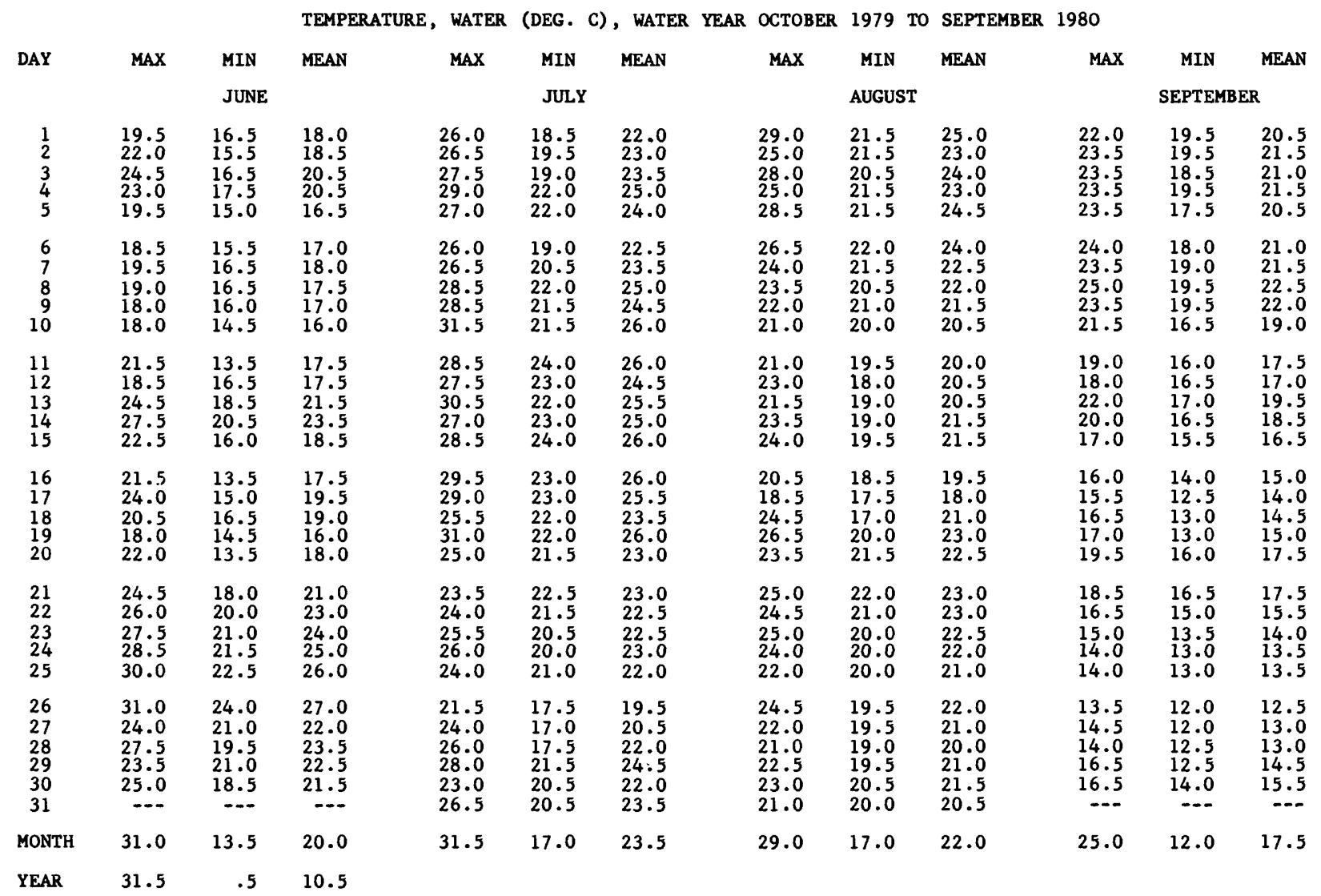


Table 16. Suspended-sediement loads for Onion River at Hingham, 1979 and 1980 water years

SEDIMENT DISCHARGE, SUSPENDED (TONS/DAY), WATER YEAR OCTOBER 1978 TO SEPTEMBER 1979

\begin{tabular}{|c|c|c|c|c|c|c|c|c|c|c|c|}
\hline $\mathrm{DAY}$ & OCT & Nov & DEC & JAN & FEB & MAR & APR & MAY & JUN & JUL & AUG \\
\hline $\begin{array}{l}1 \\
2 \\
3 \\
4 \\
5\end{array}$ & $\begin{array}{c}11 \\
10 \\
9.0 \\
8.0 \\
8.0\end{array}$ & $\begin{array}{l}3.0 \\
3.0 \\
3.0 \\
3.0 \\
3.0\end{array}$ & $\begin{array}{l}7.0 \\
6.0 \\
5.0 \\
4.0 \\
4.0\end{array}$ & $\begin{array}{l}.11 \\
.11 \\
.11 \\
.11 \\
.11\end{array}$ & $\begin{array}{l}.06 \\
.06 \\
.06 \\
.06 \\
.06\end{array}$ & $\begin{array}{l}.51 \\
1.1 \\
2.4 \\
4.5 \\
4.3\end{array}$ & $\begin{array}{c}8.9 \\
4.0 \\
3.7 \\
19 \\
45\end{array}$ & $\begin{array}{l}11 \\
9.1 \\
28 \\
11\end{array}$ & $\begin{array}{l}6.6 \\
5.4 \\
4.8 \\
6.6 \\
6.2\end{array}$ & $\begin{array}{c}15 \\
12 \\
6.9 \\
8.5 \\
10\end{array}$ & $\begin{array}{r}5.6 \\
4.7 \\
4.0 \\
3.8 \\
11\end{array}$ \\
\hline $\begin{array}{r}6 \\
7 \\
8 \\
9 \\
10\end{array}$ & $\begin{array}{c}12 \\
10 \\
9.0 \\
9.0 \\
8.0\end{array}$ & $\begin{array}{l}3.0 \\
3.0 \\
3.0 \\
3.0 \\
3.0\end{array}$ & $\begin{array}{l}3.6 \\
3.0 \\
2.5 \\
2.1 \\
1.6\end{array}$ & $\begin{array}{l}.11 \\
.14 \\
.18 \\
.21 \\
.25\end{array}$ & $\begin{array}{l}.06 \\
.06 \\
.03 \\
.03 \\
.03\end{array}$ & $\begin{array}{l}3.6 \\
3.0 \\
2.4 \\
1.9 \\
1.6\end{array}$ & $\begin{array}{c}36 \\
21 \\
15 \\
4.7 \\
1.4\end{array}$ & $\begin{array}{l}6.9 \\
6.1 \\
6.2 \\
6.2 \\
6.1\end{array}$ & $\begin{array}{l}5.8 \\
7.8 \\
6.5 \\
7.4 \\
20\end{array}$ & $\begin{array}{l}7.3 \\
6.0 \\
4.2 \\
2.9 \\
3.3\end{array}$ & $\begin{array}{r}6.0 \\
3.9 \\
2.8 \\
34 \\
112\end{array}$ \\
\hline $\begin{array}{l}11 \\
12 \\
13 \\
14 \\
15\end{array}$ & $\begin{array}{l}8.0 \\
7.0 \\
6.0 \\
6.0 \\
6.0\end{array}$ & $\begin{array}{l}3.0 \\
3.0 \\
3.0 \\
4.0 \\
5.0\end{array}$ & $\begin{array}{l}1.4 \\
1.1 \\
.92 \\
.78 \\
.64\end{array}$ & $\begin{array}{l}.32 \\
.42 \\
.49 \\
. .62 \\
.78\end{array}$ & $\begin{array}{l}.03 \\
.03 \\
.03 \\
.03 \\
.03\end{array}$ & $\begin{array}{l}1.6 \\
6.2 \\
7.0 \\
7.1 \\
7.3\end{array}$ & $\begin{array}{c}2.7 \\
193 \\
49 \\
17 \\
7.5\end{array}$ & $\begin{array}{l}6.7 \\
6.7 \\
7.4 \\
8.8 \\
9.3\end{array}$ & $\begin{array}{c}10 \\
7.0 \\
5.8 \\
5.1 \\
4.8\end{array}$ & $\begin{array}{l}4.7 \\
2.9 \\
5.4 \\
4.9 \\
4.1\end{array}$ & $\begin{array}{c}18 \\
9.2 \\
6.9 \\
6.2 \\
4.8\end{array}$ \\
\hline $\begin{array}{l}16 \\
17 \\
18 \\
19 \\
20\end{array}$ & $\begin{array}{l}6.0 \\
6.0 \\
6.0 \\
6.0 \\
6.0\end{array}$ & $\begin{array}{l}5.0 \\
8.0 \\
15 \\
13 \\
11\end{array}$ & $\begin{array}{l}.52 \\
.43 \\
.35 \\
.30 \\
.26\end{array}$ & $\begin{array}{c}1.0 \\
1.2 \\
1.1 \\
.87 \\
.68\end{array}$ & $\begin{array}{l}.03 \\
.03 \\
.03 \\
.03 \\
.04\end{array}$ & $\begin{array}{l}6.8 \\
6.6 \\
8.2 \\
18 \\
29\end{array}$ & $\begin{array}{l}6.0 \\
5.7 \\
5.6 \\
5.6 \\
5.7\end{array}$ & $\begin{array}{c}8.3 \\
7.7 \\
9.1 \\
17 \\
14\end{array}$ & $\begin{array}{l}4.4 \\
4.4 \\
4.1 \\
4.1 \\
7.4\end{array}$ & $\begin{array}{l}3.8 \\
3.7 \\
4.0 \\
4.0 \\
4.0\end{array}$ & $\begin{array}{r}4.1 \\
4.4 \\
4.7 \\
4.8 \\
12\end{array}$ \\
\hline $\begin{array}{l}21 \\
22 \\
23 \\
24 \\
25\end{array}$ & $\begin{array}{l}6.0 \\
5.0 \\
3.0 \\
4.0 \\
4.0\end{array}$ & $\begin{array}{l}{ }_{9.0}^{10} \\
11 \\
12 \\
11\end{array}$ & $\begin{array}{l}.22 \\
.16 \\
.16 \\
.12 \\
.12\end{array}$ & $\begin{array}{l}.53 \\
.42 \\
.28 \\
.21 \\
.18\end{array}$ & $\begin{array}{l}.04 \\
.04 \\
.04 \\
.04 \\
.04\end{array}$ & $\begin{array}{l}23 \\
17 \\
46 \\
49 \\
18\end{array}$ & $\begin{array}{l}6.1 \\
6.0 \\
4.4 \\
3.0 \\
9.1\end{array}$ & $\begin{array}{r}11 \\
8.1 \\
6.5 \\
5.5 \\
4.4\end{array}$ & $\begin{array}{l}7.0 \\
5.4 \\
4.1 \\
3.5 \\
3.7\end{array}$ & $\begin{array}{l}3.6 \\
3.2 \\
3.2 \\
3.9 \\
8.4\end{array}$ & $\begin{array}{l}10 \\
7.9 \\
20 \\
17 \\
8.0\end{array}$ \\
\hline $\begin{array}{l}26 \\
27 \\
28 \\
29 \\
30 \\
31\end{array}$ & $\begin{array}{l}4.0 \\
4.0 \\
3.0 \\
3.0 \\
2.0 \\
2.0\end{array}$ & $\begin{array}{c}10 \\
9.0 \\
8.0 \\
8.0 \\
8.0 \\
--.\end{array}$ & $\begin{array}{l}.11 \\
.11 \\
.11 \\
.11 \\
.11 \\
.11\end{array}$ & $\begin{array}{l}.14 \\
.11 \\
.06 \\
.06 \\
.06 \\
.06\end{array}$ & $\begin{array}{l}.04 \\
.09 \\
.19 \\
--- \\
--- \\
--\end{array}$ & $\begin{array}{r}9.2 \\
7.6 \\
6.6 \\
8.1 \\
388 \\
86\end{array}$ & $\begin{array}{l}23 \\
18 \\
10 \\
7.9 \\
12 \\
-. .\end{array}$ & $\begin{array}{l}4.1 \\
4.1 \\
4.4 \\
4.7 \\
4.8 \\
9.4\end{array}$ & $\begin{array}{l}3.7 \\
3.9 \\
4.2 \\
48 \\
12 \\
\end{array}$ & $\begin{array}{l}6.6 \\
4.8 \\
4.4 \\
3.8 \\
5.7 \\
7.4\end{array}$ & $\begin{array}{l}5.7 \\
5.6 \\
5.4 \\
5.7 \\
7.1 \\
8.0\end{array}$ \\
\hline TOTAL & 197.0 & 196.0 & 46.94 & 11.03 & 1.34 & 781.61 & 556.0 & 273.6 & 229.7 & 172.6 & 363.3 \\
\hline
\end{tabular}

WTR YR 1979 TOTAL 2943.92

SEDIMENT DISCHARGE, SUSPENDED (TONS/DAY), WATER YEAR OCTOBER 1979 TO SEPTEMBER 1980

\begin{tabular}{|c|c|c|c|c|c|c|c|c|c|c|c|c|}
\hline DAY & OCT & Nov & DEC & JAN & FEB & MAR & APR & MAY & JUN & JUL & AUG & SEP \\
\hline $\begin{array}{l}1 \\
2 \\
3 \\
4 \\
5\end{array}$ & $\begin{array}{l}2.5 \\
2.7 \\
2.4 \\
2.4 \\
2.3\end{array}$ & $\begin{array}{l}5.8 \\
5.0 \\
4.3 \\
3.4 \\
3.4\end{array}$ & $\begin{array}{c}1.5 \\
.64 \\
.48 \\
.59 \\
.69\end{array}$ & $\begin{array}{c}1.4 \\
1.2 \\
1.1 \\
.97 \\
.86\end{array}$ & $\begin{array}{r}.95 \\
1.1 \\
.91 \\
.81 \\
.72\end{array}$ & $\begin{array}{l}.19 \\
.16 \\
.13 \\
.13 \\
.09\end{array}$ & $\begin{array}{l}.52 \\
1.52 \\
17 \\
10\end{array}$ & $\begin{array}{l}9.3 \\
6.7 \\
4.9 \\
3.8 \\
3.9\end{array}$ & $\begin{array}{c}13 \\
11 \\
9.8 \\
8.5 \\
51\end{array}$ & $\begin{array}{l}1.8 \\
1.8 \\
1.8 \\
1.9 \\
2.6\end{array}$ & $\begin{array}{l}1.5 \\
1.6 \\
1.7 \\
1.5 \\
1.8\end{array}$ & $\begin{array}{l}4.9 \\
5.6 \\
3.9 \\
3.8 \\
3.3\end{array}$ \\
\hline $\begin{array}{r}6 \\
7 \\
8 \\
9 \\
10\end{array}$ & $\begin{array}{l}2.4 \\
2.6 \\
2.6 \\
2.4 \\
2.0\end{array}$ & $\begin{array}{l}4.2 \\
3.9 \\
3.4 \\
2.7 \\
2.2\end{array}$ & $\begin{array}{l}.85 \\
1.0 \\
1.0 \\
5.0 \\
6.0\end{array}$ & $\begin{array}{l}.81 \\
.81 \\
.76 \\
.67 \\
.67\end{array}$ & $\begin{array}{l}.60 \\
.53 \\
.45 \\
.34 \\
.30\end{array}$ & $\begin{array}{l}.09 \\
.05 \\
.05 \\
.06 \\
.10\end{array}$ & $\begin{array}{r}1.8 \\
5.9 \\
122 \\
44 \\
9.6\end{array}$ & $\begin{array}{l}5.0 \\
5.4 \\
6.6 \\
5.9 \\
5.6\end{array}$ & $\begin{array}{c}34 \\
42 \\
56 \\
30 \\
8.5\end{array}$ & $\begin{array}{l}2.3 \\
2.1 \\
1.9 \\
1.8 \\
1.5\end{array}$ & $\begin{array}{l}1.6 \\
2.3 \\
55 \\
23 \\
11\end{array}$ & $\begin{array}{l}3.2 \\
3.1 \\
2.7 \\
3.1 \\
2.8\end{array}$ \\
\hline $\begin{array}{l}11 \\
12 \\
13 \\
14 \\
15\end{array}$ & $\begin{array}{l}2.0 \\
2.0 \\
1.5 \\
1.2 \\
1.4\end{array}$ & $\begin{array}{l}3.0 \\
1.5 \\
1.4 \\
1.2 \\
1.3\end{array}$ & $\begin{array}{l}8.0 \\
4.0 \\
.70 \\
.45 \\
.46\end{array}$ & $\begin{array}{l}.67 \\
.62 \\
.62 \\
.56 \\
.56\end{array}$ & $\begin{array}{l}.30 \\
.28 \\
.24 \\
.24 \\
.20\end{array}$ & $\begin{array}{l}.15 \\
.18 \\
.21 \\
.28 \\
.38\end{array}$ & $\begin{array}{l}3.9 \\
3.4 \\
2.9 \\
2.6 \\
4.1\end{array}$ & $\begin{array}{l}6.8 \\
5.3 \\
11 \\
18 \\
18\end{array}$ & $\begin{array}{l}5.6 \\
6.4 \\
5.5 \\
7.9 \\
8.7\end{array}$ & $\begin{array}{l}1.3 \\
1.2 \\
1.4 \\
1.8 \\
2.8\end{array}$ & $\begin{array}{l}6.9 \\
6.4 \\
5.7 \\
5.6 \\
5.2\end{array}$ & $\begin{array}{l}2.5 \\
3.4 \\
7.4 \\
7.6 \\
4.1\end{array}$ \\
\hline $\begin{array}{l}16 \\
17 \\
18 \\
19 \\
20\end{array}$ & $\begin{array}{l}1.4 \\
1.5 \\
1.6 \\
3.0 \\
2.1\end{array}$ & $\begin{array}{l}1.2 \\
1.2 \\
1.1 \\
1.1 \\
1.1\end{array}$ & $\begin{array}{l}.38 \\
.42 \\
.46 \\
.53 \\
.63\end{array}$ & $\begin{array}{r}3.6 \\
14 \\
4.7 \\
1.9 \\
1.3\end{array}$ & $\begin{array}{r}.19 \\
.19 \\
.18 \\
.23 \\
.26\end{array}$ & $\begin{array}{l}1.8 \\
2.9 \\
2.7 \\
1.7 \\
1.0\end{array}$ & $\begin{array}{l}6.6 \\
4.6 \\
5.1 \\
6.0 \\
7.0\end{array}$ & $\begin{array}{l}15 \\
12 \\
21 \\
27 \\
20\end{array}$ & $\begin{array}{l}4.8 \\
3.5 \\
2.4 \\
7.2 \\
8.4\end{array}$ & $\begin{array}{r}5.1 \\
4.2 \\
2.8 \\
2.4 \\
57\end{array}$ & $\begin{array}{r}4.7 \\
4.9 \\
5.1 \\
5.0 \\
25\end{array}$ & $\begin{array}{l}6.2 \\
8.2\end{array}$ \\
\hline $\begin{array}{l}21 \\
22 \\
23 \\
24 \\
25\end{array}$ & $\begin{array}{c}2.2 \\
2.5 \\
12 \\
13 \\
6.2\end{array}$ & $\begin{array}{l}9.3 \\
14 \\
14 \\
11 \\
9.4\end{array}$ & $\begin{array}{r}.72 \\
2.90 \\
22 \\
47\end{array}$ & $\begin{array}{l}.97 \\
.77 \\
.68 \\
.59 \\
.62\end{array}$ & $\begin{array}{l}.28 \\
.40 \\
.41 \\
.37 \\
.32\end{array}$ & $\begin{array}{r}.92 \\
.72 \\
.54 \\
.58 \\
3.7\end{array}$ & $\begin{array}{r}8.0 \\
9.6 \\
10 \\
8.8 \\
7.2\end{array}$ & $\begin{array}{c}14 \\
12 \\
9.7 \\
8.8 \\
8.4\end{array}$ & $\begin{array}{l}4.4 \\
3.1 \\
2.6 \\
2.2 \\
2.0\end{array}$ & $\begin{array}{c}27 \\
12 \\
5.6 \\
3.8 \\
3.1\end{array}$ & $\begin{array}{c}20 \\
9.7 \\
8.3 \\
7.4 \\
7.1\end{array}$ & $\begin{array}{c}6.4 \\
203 \\
32 \\
10 \\
6.3\end{array}$ \\
\hline $\begin{array}{l}26 \\
27 \\
28 \\
29 \\
30 \\
31\end{array}$ & $\begin{array}{l}1.9 \\
1.9 \\
1.8 \\
1.8 \\
1.7 \\
1.6\end{array}$ & $\begin{array}{c}25 \\
43 \\
24 \\
14 \\
3.1 \\
---\end{array}$ & $\begin{array}{r}22 \\
8.6 \\
4.0 \\
2.4 \\
1.9 \\
1.6\end{array}$ & $\begin{array}{l}.66 \\
.69 \\
.69 \\
.70 \\
.73 \\
.84\end{array}$ & $\begin{array}{l}.34 \\
.34 \\
.32 \\
.32 \\
-.- \\
--.\end{array}$ & $\begin{array}{c}5.4 \\
2.5 \\
1.2 \\
.61 \\
.52 \\
.52\end{array}$ & $\begin{array}{r}6.2 \\
5.0 \\
4.6 \\
7.5 \\
12\end{array}$ & $\begin{array}{l}6.9 \\
4.7 \\
4.3 \\
12 \\
13 \\
15\end{array}$ & $\begin{array}{l}1.7 \\
1.3 \\
1.7 \\
1.9 \\
1.5 \\
--.\end{array}$ & $\begin{array}{l}2.7 \\
2.4 \\
2.0 \\
1.8 \\
1.6 \\
1.8\end{array}$ & $\begin{array}{c}9.8 \\
8.4 \\
26 \\
11 \\
6.2 \\
4.9\end{array}$ & $\begin{array}{l}5.9 \\
5.0 \\
4.3 \\
4.0 \\
3.9\end{array}$ \\
\hline TAL & 88.6 & 219.2 & 147.00 & 45.72 & 12.12 & 29.56 & 338.24 & 320.0 & 346.6 & 163.3 & 294.3 & 373 \\
\hline
\end{tabular}

WTR YR 1980 TOTAL 2378.24 
Table 17. Suspended-sediment loads for Onion River near Sheboygan Falls, 1979 and 1980 water years SEDIMENT DISCHARGE, SUSPENDED (TONS/DAY), WATER YEAR OCTOBER 1978 TO SEPTEMBER 1979

\begin{tabular}{|c|c|c|c|c|c|c|c|c|c|c|c|c|}
\hline DAY & OCT & Nov & DEC & JAN & FEB & MAR & APR & MAY & JUN & JUL & AUG & SEP \\
\hline $\begin{array}{l}1 \\
2 \\
3 \\
4 \\
5\end{array}$ & $\begin{array}{c}23 \\
18 \\
12 \\
8.0 \\
6.0\end{array}$ & $\begin{array}{l}2.0 \\
2.0 \\
2.0 \\
2.0 \\
2.0\end{array}$ & $\begin{array}{l}6.0 \\
5.0 \\
4.0 \\
3.0 \\
4.0\end{array}$ & $\begin{array}{l}.77 \\
.65 \\
.83 \\
.96 \\
1.1\end{array}$ & $\begin{array}{l}.05 \\
.09 \\
.14 \\
.14 \\
.23\end{array}$ & $\begin{array}{l}.27 \\
.37 \\
.70 \\
1.1 \\
1.3\end{array}$ & $\begin{array}{r}189 \\
63 \\
28 \\
27 \\
33\end{array}$ & $\begin{array}{l}59 \\
19 \\
19 \\
13 \\
11\end{array}$ & $\begin{array}{l}6.9 \\
7.2 \\
3.3 \\
7.5 \\
5.2\end{array}$ & $\begin{array}{l}39 \\
36 \\
24 \\
15 \\
12\end{array}$ & $\begin{array}{l}7.2 \\
4.6 \\
2.9 \\
2.3 \\
2.5\end{array}$ & $\begin{array}{l}13 \\
11 \\
8.6 \\
6.7 \\
5.7\end{array}$ \\
\hline $\begin{array}{r}6 \\
7 \\
8 \\
9 \\
10\end{array}$ & $\begin{array}{l}27 \\
25 \\
20 \\
16 \\
13\end{array}$ & $\begin{array}{l}2.0 \\
2.0 \\
2.0 \\
2.0 \\
2.0\end{array}$ & $\begin{array}{l}2.6 \\
2.2 \\
1.9 \\
1.7 \\
1.5\end{array}$ & $\begin{array}{l}1.4 \\
1.6 \\
1.6 \\
1.6 \\
1.5\end{array}$ & $\begin{array}{l}.32 \\
.44 \\
.58 \\
.51 \\
.43\end{array}$ & $\begin{array}{l}1.4 \\
1.7 \\
1.8 \\
2.0 \\
2.3\end{array}$ & $\begin{array}{l}24 \\
11 \\
4.5 \\
4.1 \\
5.2\end{array}$ & $\begin{array}{l}8.7 \\
8.1 \\
7.7 \\
7.6 \\
7.8\end{array}$ & $\begin{array}{l}4.6 \\
14 \\
13 \\
8.0 \\
60\end{array}$ & $\begin{array}{l}10 \\
7.1 \\
5.8 \\
5.1 \\
4.6\end{array}$ & $\begin{array}{c}10 \\
10 \\
7.0 \\
7.0 \\
258\end{array}$ & $\begin{array}{l}5.1 \\
4.2 \\
3.1 \\
3.0 \\
2.9\end{array}$ \\
\hline $\begin{array}{l}11 \\
12 \\
13 \\
14 \\
15\end{array}$ & $\begin{array}{r}10 \\
9.0 \\
7.0 \\
6.0 \\
5.0\end{array}$ & $\begin{array}{l}2.0 \\
2.0 \\
3.0 \\
4.0 \\
5.0\end{array}$ & $\begin{array}{l}1.7 \\
2.0 \\
2.4 \\
2.8 \\
3.4\end{array}$ & $\begin{array}{l}1.5 \\
1.4 \\
1.4 \\
1.0 \\
.84\end{array}$ & $\begin{array}{l}.32 \\
.27 \\
.22 \\
.16 \\
.11\end{array}$ & $\begin{array}{l}2.5 \\
2.9 \\
3.3 \\
3.6 \\
3.9\end{array}$ & $\begin{array}{c}6.8 \\
769 \\
382 \\
145 \\
47\end{array}$ & $\begin{array}{c}8.1 \\
8.9 \\
9.3 \\
10 \\
13\end{array}$ & $\begin{array}{l}38 \\
25 \\
19 \\
14 \\
13\end{array}$ & $\begin{array}{l}5.6 \\
6.5 \\
5.0 \\
8.8 \\
6.4\end{array}$ & $\begin{array}{r}229 \\
80 \\
34 \\
20 \\
13\end{array}$ & $\begin{array}{l}3.1 \\
3.4 \\
3.4 \\
3.3 \\
2.4\end{array}$ \\
\hline $\begin{array}{l}16 \\
17 \\
18 \\
19 \\
20\end{array}$ & $\begin{array}{l}6.0 \\
6.0 \\
5.0 \\
5.0 \\
5.0\end{array}$ & $\begin{array}{l}4.0 \\
10 \\
27 \\
23 \\
16\end{array}$ & $\begin{array}{l}4.2 \\
4.9 \\
4.6 \\
4.2 \\
3.9\end{array}$ & $\begin{array}{l}.68 \\
.14 \\
.12 \\
.09 \\
.10\end{array}$ & $\begin{array}{l}.10 \\
.05 \\
.05 \\
.05 \\
.05\end{array}$ & $\begin{array}{l}4.2 \\
4.6 \\
7.8 \\
29 \\
56\end{array}$ & $\begin{array}{l}26 \\
20 \\
16 \\
14 \\
12\end{array}$ & $\begin{array}{l}10 \\
12 \\
14 \\
20 \\
16\end{array}$ & $\begin{array}{l}11 \\
10 \\
9.0 \\
8.5 \\
25\end{array}$ & $\begin{array}{l}4.5 \\
3.5 \\
2.9 \\
2.9 \\
2.8\end{array}$ & $\begin{array}{r}8.6 \\
8.2 \\
7.9 \\
7.1 \\
20\end{array}$ & $\begin{array}{c}1.7 \\
1.2 \\
.66 \\
.34 \\
.16\end{array}$ \\
\hline $\begin{array}{l}21 \\
22 \\
23 \\
24 \\
25\end{array}$ & $\begin{array}{l}5.0 \\
4.0 \\
2.0 \\
5.0 \\
6.0\end{array}$ & $\begin{array}{l}11 \\
8.0 \\
12 \\
27 \\
21\end{array}$ & $\begin{array}{l}3.4 \\
3.1 \\
2.8 \\
2.5 \\
2.3\end{array}$ & $\begin{array}{l}.10 \\
.05 \\
.05 \\
.05 \\
.05\end{array}$ & $\begin{array}{l}.06 \\
.06 \\
.07 \\
.07 \\
.08\end{array}$ & $\begin{array}{r}239 \\
327 \\
463 \\
189 \\
70\end{array}$ & $\begin{array}{r}11 \\
9.9 \\
8.2 \\
6.9 \\
9.8\end{array}$ & $\begin{array}{l}13 \\
11 \\
9.3 \\
8.5 \\
7.6\end{array}$ & $\begin{array}{l}19 \\
11 \\
7.2 \\
6.5 \\
6.0\end{array}$ & $\begin{array}{l}3.0 \\
2.9 \\
2.7 \\
2.4 \\
3.7\end{array}$ & $\begin{array}{r}72 \\
41 \\
41 \\
110 \\
72\end{array}$ & $\begin{array}{l}.09 \\
.10 \\
.13 \\
.18 \\
.25\end{array}$ \\
\hline $\begin{array}{l}26 \\
27 \\
28 \\
29 \\
30 \\
31\end{array}$ & $\begin{array}{l}5.0 \\
4.0 \\
3.0 \\
2.0 \\
2.0 \\
2.0\end{array}$ & $\begin{array}{r}16 \\
12 \\
10 \\
8.0 \\
7.0 \\
-\end{array}$ & $\begin{array}{c}1.9 \\
1.6 \\
1.4 \\
1.2 \\
1.1 \\
.93\end{array}$ & $\begin{array}{l}.05 \\
.05 \\
.05 \\
.05 \\
.05 \\
.05\end{array}$ & $\begin{array}{l}.08 \\
.16 \\
.17 \\
--- \\
--- \\
---\end{array}$ & $\begin{array}{r}50 \\
37 \\
25 \\
19 \\
732 \\
787\end{array}$ & $\begin{array}{l}82 \\
61 \\
29 \\
15 \\
98 \\
---\end{array}$ & $\begin{array}{r}7.1 \\
6.9 \\
6.8 \\
6.9 \\
9.0 \\
10\end{array}$ & $\begin{array}{l}5.8 \\
5.7 \\
5.5 \\
29 \\
66\end{array}$ & $\begin{array}{c}10 \\
8.6 \\
6.2 \\
4.7 \\
3.8 \\
6.3\end{array}$ & $\begin{array}{l}33 \\
24 \\
28 \\
21 \\
17 \\
15\end{array}$ & $\begin{array}{r}.36 \\
.50 \\
.65 \\
.87 \\
1.3\end{array}$ \\
\hline TOTAL & 272.0 & 248.0 & 88.23 & 19.88 & 5.06 & 3068.74 & 2157.4 & 378.3 & 463.9 & 261.8 & 1213.3 & 87.39 \\
\hline
\end{tabular}

WTR YR 1979 TOTAL 8264.00

SEDIMENT DISCHARGE, SUSPENDED (TONS/DAY), WATER YEAR OCTOBER 1979 TO SEPTEMBER 1980

\begin{tabular}{|c|c|c|c|c|c|c|c|c|c|c|c|c|}
\hline DAY & OCT & Nov & $\mathrm{DEC}$ & JAN & FEB & MAR & $A D R$ & MAY & JUN & JUL & AUG & SEP \\
\hline $\begin{array}{l}1 \\
2 \\
3 \\
4 \\
5\end{array}$ & $\begin{array}{l}1.5 \\
1.5 \\
1.7 \\
1.6 \\
1.8\end{array}$ & $\begin{array}{l}2.6 \\
2.5 \\
1.9 \\
1.5 \\
1.2\end{array}$ & $\begin{array}{l}7.2 \\
6.1 \\
5.7 \\
5.6 \\
5.5\end{array}$ & $\begin{array}{l}1.8 \\
1.8 \\
1.9 \\
2.0 \\
2.1\end{array}$ & $\begin{array}{l}9.7 \\
12 \\
10 \\
8.7 \\
7.2\end{array}$ & $\begin{array}{l}.20 \\
.20 \\
.30 \\
.30 \\
.50\end{array}$ & $\begin{array}{r}5.3 \\
5.2 \\
5.7 \\
35 \\
115\end{array}$ & $\begin{array}{l}11 \\
11 \\
10 \\
9.0 \\
8.6\end{array}$ & $\begin{array}{l}13 \\
12 \\
12 \\
11 \\
17\end{array}$ & $\begin{array}{l}6.1 \\
6.0 \\
6.2 \\
6.1 \\
5.9\end{array}$ & $\begin{array}{l}5.8 \\
5.5 \\
5.9 \\
6.1 \\
6.1\end{array}$ & $\begin{array}{r}12 \\
12 \\
11 \\
8.9 \\
8.3\end{array}$ \\
\hline $\begin{array}{r}6 \\
7 \\
8 \\
9 \\
10\end{array}$ & $\begin{array}{l}1.9 \\
1.9 \\
1.9 \\
2.0 \\
1.8\end{array}$ & $\begin{array}{c}.99 \\
1.0 \\
.84 \\
.78 \\
.75\end{array}$ & $\begin{array}{l}5.4 \\
5.6 \\
6.0 \\
8.4 \\
5.7\end{array}$ & $\begin{array}{l}2.0 \\
2.0 \\
1.9 \\
1.8 \\
1.8\end{array}$ & $\begin{array}{l}5.8 \\
4.6 \\
3.5 \\
2.6 \\
2.0\end{array}$ & $\begin{array}{l}.60 \\
.60 \\
.80 \\
.80 \\
.80\end{array}$ & $\begin{array}{r}19 \\
13 \\
143 \\
211 \\
86\end{array}$ & $\begin{array}{l}8.0 \\
7.8 \\
7.6 \\
8.0 \\
8.0\end{array}$ & $\begin{array}{l}167 \\
210 \\
195 \\
141 \\
108\end{array}$ & $\begin{array}{l}6.2 \\
5.7 \\
4.7 \\
3.9 \\
3.7\end{array}$ & $\begin{array}{r}6.8 \\
6.7 \\
140 \\
213 \\
104\end{array}$ & $\begin{array}{l}7.4 \\
6.3 \\
5.3 \\
4.9 \\
4.9\end{array}$ \\
\hline $\begin{array}{l}11 \\
12 \\
13 \\
14 \\
15\end{array}$ & $\begin{array}{l}1.6 \\
1.4 \\
1.4 \\
1.2 \\
1.1\end{array}$ & $\begin{array}{l}.77 \\
.85 \\
.86 \\
.94 \\
.96\end{array}$ & $\begin{array}{l}4.9 \\
4.3 \\
3.7 \\
3.3 \\
2.9\end{array}$ & $\begin{array}{l}1.7 \\
1.7 \\
1.7 \\
1.7 \\
1.8\end{array}$ & $\begin{array}{l}1.7 \\
1.4 \\
1.2 \\
1.0 \\
.90\end{array}$ & $\begin{array}{l}.70 \\
.60 \\
.60 \\
.60 \\
.60\end{array}$ & $\begin{array}{l}36 \\
25 \\
18 \\
13 \\
12\end{array}$ & $\begin{array}{l}8.5 \\
8.9 \\
11 \\
22 \\
23\end{array}$ & $\begin{array}{l}48 \\
31 \\
24 \\
22 \\
23\end{array}$ & $\begin{array}{l}3.6 \\
3.4 \\
3.2 \\
3.1 \\
8.3\end{array}$ & $\begin{array}{l}60 \\
39 \\
36 \\
20 \\
11\end{array}$ & $\begin{array}{l}3.9 \\
3.4 \\
4.3 \\
5.1 \\
4.9\end{array}$ \\
\hline $\begin{array}{l}16 \\
17 \\
18 \\
19 \\
20\end{array}$ & $\begin{array}{l}1.3 \\
1.3 \\
1.3 \\
1.4 \\
4.8\end{array}$ & $\begin{array}{l}1.7 \\
3.7 \\
3.8 \\
3.1 \\
2.5\end{array}$ & $\begin{array}{l}2.5 \\
2.3 \\
2.1 \\
1.9 \\
1.9\end{array}$ & $\begin{array}{l}11 \\
63 \\
30 \\
11 \\
4.7\end{array}$ & $\begin{array}{r}.80 \\
.80 \\
.80 \\
.90 \\
1.9\end{array}$ & $\begin{array}{l}1.0 \\
5.1 \\
7.9 \\
28 \\
14\end{array}$ & $\begin{array}{l}20 \\
17 \\
16 \\
15 \\
15\end{array}$ & $\begin{array}{l}17 \\
12 \\
12 \\
22 \\
21\end{array}$ & $\begin{array}{l}22 \\
16 \\
13 \\
14 \\
31\end{array}$ & $\begin{array}{c}15 \\
15 \\
7.9 \\
5.0 \\
180\end{array}$ & $\begin{array}{l}7.5 \\
6.1 \\
6.3 \\
6.4 \\
67\end{array}$ & $\begin{array}{l}4.3 \\
24 \\
20 \\
13 \\
17\end{array}$ \\
\hline $\begin{array}{l}21 \\
22 \\
23 \\
24 \\
25\end{array}$ & $\begin{array}{l}2.7 \\
2.2 \\
2.5 \\
3.1 \\
3.2\end{array}$ & $\begin{array}{l}2.3 \\
2.7 \\
2.8 \\
2.1 \\
2.3\end{array}$ & $\begin{array}{r}1.8 \\
1.7 \\
2.2 \\
5.2 \\
73\end{array}$ & $\begin{array}{l}4.2 \\
5.1 \\
6.7 \\
6.6 \\
6.1\end{array}$ & $\begin{array}{l}2.5 \\
3.2 \\
4.4 \\
2.7 \\
1.5\end{array}$ & $\begin{array}{l}3.3 \\
1.9 \\
1.4 \\
1.2 \\
1.2\end{array}$ & $\begin{array}{l}15 \\
15 \\
15 \\
12 \\
9.5\end{array}$ & $\begin{array}{c}13 \\
10 \\
8.9 \\
8.0 \\
7.0\end{array}$ & $\begin{array}{l}24 \\
16 \\
13 \\
11 \\
9.4\end{array}$ & $\begin{array}{r}232 \\
99 \\
48 \\
24 \\
16\end{array}$ & $\begin{array}{l}49 \\
38 \\
24 \\
16 \\
13\end{array}$ & $\begin{array}{r}32 \\
843 \\
472 \\
256 \\
133\end{array}$ \\
\hline $\begin{array}{l}26 \\
27 \\
28 \\
29 \\
30 \\
31\end{array}$ & $\begin{array}{l}2.4 \\
2.0 \\
2.1 \\
2.3 \\
2.2 \\
2.4\end{array}$ & $\begin{array}{c}5.3 \\
25 \\
23 \\
14 \\
8.6 \\
- \\
-\end{array}$ & $\begin{array}{r}29 \\
7.5 \\
3.6 \\
2.1 \\
1.7 \\
1.8\end{array}$ & $\begin{array}{l}5.9 \\
6.0 \\
6.4 \\
6.8 \\
7.2 \\
8.2\end{array}$ & $\begin{array}{r}1.0 \\
.80 \\
.40 \\
.30 \\
--- \\
---\end{array}$ & $\begin{array}{l}1.5 \\
2.2 \\
4.6 \\
7.1 \\
8.4 \\
6.5\end{array}$ & $\begin{array}{l}7.5 \\
6.1 \\
6.2 \\
7.2 \\
9.5 \\
---\end{array}$ & $\begin{array}{c}6.4 \\
6.2 \\
6.3 \\
6.3 \\
10 \\
13\end{array}$ & $\begin{array}{l}8.4 \\
6.9 \\
6.9 \\
7.6 \\
7.4 \\
---\end{array}$ & $\begin{array}{c}13 \\
11 \\
9.7 \\
7.8 \\
6.7 \\
5.8\end{array}$ & $\begin{array}{l}16 \\
17 \\
14 \\
18 \\
16 \\
13\end{array}$ & $\begin{array}{l}61 \\
37 \\
26 \\
19 \\
18\end{array}$ \\
\hline TOTAL & 61.5 & 121.34 & 220.6 & 216.6 & 94.30 & 103.50 & 928.2 & 341.5 & 1240.6 & 772.0 & 993.2 & 2077.9 \\
\hline
\end{tabular}

WTR YR 1980 TOTAL 7171.24 
Table 18. Total organic nitrogen load, in pounds per day, for Onion River at Hingham, 1979 and 1980 water years

TOTAL ORGANIC NITROGEN, WATER YEAR OCTOBER 1978 TO SEPTEMBER 1979

\begin{tabular}{|c|c|c|c|c|c|c|c|c|c|c|c|c|}
\hline DAY & UCT & NOV & $D E C$ & JAN & FEH & MAR & $\triangle P R$ & MAY & JUN & JUL & AUG & SEP \\
\hline $\begin{array}{l}1 \\
2 \\
3 \\
4 \\
5\end{array}$ & $\begin{array}{r}120 \\
110 \\
94 \\
8 R \\
84\end{array}$ & $\begin{array}{l}20 \\
20 \\
20 \\
20 \\
20\end{array}$ & $\begin{array}{l}64 \\
62 \\
60 \\
56 \\
54\end{array}$ & $\begin{array}{l}38 \\
38 \\
38 \\
35 \\
35\end{array}$ & $\begin{array}{l}24 \\
24 \\
24 \\
24 \\
24\end{array}$ & $\begin{array}{l}36 \\
38 \\
40 \\
42 \\
44\end{array}$ & $\begin{array}{l}664 \\
390 \\
320 \\
410 \\
480\end{array}$ & $\begin{array}{l}470 \\
420 \\
670 \\
560 \\
390\end{array}$ & $\begin{array}{l}170 \\
150 \\
130 \\
170 \\
160\end{array}$ & $\begin{array}{l}320 \\
260 \\
140 \\
150 \\
150\end{array}$ & $\begin{array}{r}78 \\
72 \\
66 \\
66 \\
110\end{array}$ & $\begin{array}{l}76 \\
76 \\
55 \\
48 \\
48\end{array}$ \\
\hline $\begin{array}{r}6 \\
7 \\
8 \\
9 \\
10\end{array}$ & $\begin{array}{r}140 \\
110 \\
94 \\
92 \\
84\end{array}$ & $\begin{array}{l}20 \\
22 \\
22 \\
22 \\
26\end{array}$ & $\begin{array}{l}54 \\
54 \\
54 \\
50 \\
48\end{array}$ & $\begin{array}{l}35 \\
35 \\
35 \\
35 \\
35\end{array}$ & $\begin{array}{l}24 \\
24 \\
26 \\
26 \\
26\end{array}$ & $\begin{array}{l}45 \\
45 \\
45 \\
44 \\
44\end{array}$ & $\begin{array}{l}400 \\
240 \\
190 \\
180 \\
180\end{array}$ & $\begin{array}{l}330 \\
300 \\
270 \\
250 \\
230\end{array}$ & $\begin{array}{l}160 \\
190 \\
160 \\
180 \\
280\end{array}$ & $\begin{array}{r}110 \\
86 \\
72 \\
72 \\
86\end{array}$ & $\begin{array}{r}100 \\
78 \\
57 \\
444 \\
1530\end{array}$ & $\begin{array}{l}36 \\
36 \\
36 \\
36\end{array}$ \\
\hline $\begin{array}{l}11 \\
12 \\
13 \\
14 \\
15\end{array}$ & $\begin{array}{l}74 \\
70 \\
60 \\
60 \\
56\end{array}$ & $\begin{array}{l}26 \\
22 \\
26 \\
33 \\
40\end{array}$ & $\begin{array}{l}48 \\
46 \\
46 \\
46 \\
46\end{array}$ & $\begin{array}{l}35 \\
35 \\
32 \\
32 \\
32\end{array}$ & $\begin{array}{l}26 \\
26 \\
26 \\
26 \\
26\end{array}$ & $\begin{array}{l}42 \\
42 \\
42 \\
44 \\
45\end{array}$ & $\begin{array}{r}180 \\
1320 \\
1040 \\
560 \\
370\end{array}$ & $\begin{array}{l}220 \\
210 \\
200 \\
220 \\
220\end{array}$ & $\begin{array}{l}220 \\
180 \\
160 \\
140 \\
130\end{array}$ & $\begin{array}{r}120 \\
92 \\
160 \\
120 \\
86\end{array}$ & $\begin{array}{l}705 \\
348 \\
231 \\
183 \\
137\end{array}$ & 31 \\
\hline $\begin{array}{l}16 \\
17 \\
18 \\
19 \\
70\end{array}$ & $\begin{array}{l}611 \\
64 \\
52 \\
56 \\
56\end{array}$ & $\begin{array}{r}40 \\
74 \\
190 \\
150 \\
120\end{array}$ & $\begin{array}{l}42 \\
42 \\
42 \\
42 \\
42\end{array}$ & $\begin{array}{l}32 \\
32 \\
35 \\
38 \\
38\end{array}$ & $\begin{array}{l}24 \\
24 \\
26 \\
26 \\
28\end{array}$ & $\begin{array}{r}44 \\
44 \\
269 \\
512 \\
1110\end{array}$ & $\begin{array}{l}290 \\
250 \\
220 \\
200 \\
190\end{array}$ & $\begin{array}{l}190 \\
200 \\
210 \\
250 \\
250\end{array}$ & $\begin{array}{l}130 \\
130 \\
120 \\
120 \\
180\end{array}$ & $\begin{array}{l}66 \\
58 \\
52 \\
52 \\
52\end{array}$ & $\begin{array}{l}116 \\
114 \\
113 \\
109 \\
307\end{array}$ & $\begin{array}{l}24 \\
26\end{array}$ \\
\hline $\begin{array}{l}21 \\
22 \\
23 \\
24 \\
25\end{array}$ & $\begin{array}{l}52 \\
40 \\
26 \\
34 \\
36\end{array}$ & $\begin{array}{r}100 \\
98 \\
130 \\
150 \\
130\end{array}$ & $\begin{array}{l}4 ? \\
40 \\
40 \\
40 \\
40\end{array}$ & $\begin{array}{l}38 \\
38 \\
35 \\
35 \\
35\end{array}$ & $\begin{array}{l}28 \\
28 \\
30 \\
30 \\
30\end{array}$ & $\begin{array}{r}1350 \\
1320 \\
1630 \\
1720 \\
740\end{array}$ & $\begin{array}{l}180 \\
170 \\
160 \\
160 \\
250\end{array}$ & $\begin{array}{l}250 \\
210 \\
200 \\
200 \\
148\end{array}$ & $\begin{array}{l}180 \\
150 \\
130 \\
110 \\
110\end{array}$ & $\begin{array}{r}52 \\
46 \\
52 \\
52 \\
120\end{array}$ & $\begin{array}{l}480 \\
260 \\
500 \\
450 \\
250\end{array}$ & 31 \\
\hline $\begin{array}{l}26 \\
27 \\
28 \\
29 \\
30 \\
31\end{array}$ & $\begin{array}{l}34 \\
30 \\
22 \\
20 \\
16 \\
18\end{array}$ & $\begin{array}{r}110 \\
98 \\
88 \\
78 \\
74 \\
-1 .-\end{array}$ & $\begin{array}{l}38 \\
38 \\
38 \\
38 \\
38 \\
38\end{array}$ & $\begin{array}{l}35 \\
35 \\
32 \\
32 \\
30 \\
30\end{array}$ & $\begin{array}{r}32 \\
35 \\
39 \\
--- \\
---\end{array}$ & $\begin{array}{r}438 \\
374 \\
372 \\
397 \\
3640 \\
2060\end{array}$ & $\begin{array}{l}700 \\
620 \\
400 \\
330 \\
450 \\
-\cdots\end{array}$ & $\begin{array}{l}140 \\
130 \\
130 \\
130 \\
130 \\
210\end{array}$ & $\begin{array}{l}100 \\
100 \\
107 \\
391 \\
285 \\
-\cdots\end{array}$ & $\begin{array}{r}100 \\
78 \\
72 \\
58 \\
78 \\
110\end{array}$ & $\begin{array}{r}150 \\
140 \\
130 \\
130 \\
120 \\
84\end{array}$ & \\
\hline $\begin{array}{l}\operatorname{MAX} \\
\operatorname{MIN}\end{array}$ & $\begin{array}{r}140 \\
16\end{array}$ & $\begin{array}{r}190 \\
20\end{array}$ & $\begin{array}{l}64 \\
38\end{array}$ & $\begin{array}{l}38 \\
30\end{array}$ & $\begin{array}{l}39 \\
24\end{array}$ & $\begin{array}{r}3640 \\
36\end{array}$ & $\begin{array}{r}1320 \\
160\end{array}$ & $\begin{array}{l}670 \\
130\end{array}$ & $\begin{array}{l}391 \\
100\end{array}$ & $\begin{array}{r}320 \\
46\end{array}$ & $\begin{array}{r}1530 \\
57\end{array}$ & 24 \\
\hline
\end{tabular}

WTR YP 1974 MAX 3640 MIN 16 $\begin{array}{lcclllllllll}\text { TOTAL } & 1960 & 1989 & 1428 & 1075 & 756 & 16658 & 11494 & 7938 & 4923 & 3122 & 7658 \\ \text { TOTAL LOAD FOR YEAR: } & 60118 & \text { POUNDS } & & & & & & & & \end{array}$

\begin{tabular}{|c|c|c|c|c|c|c|c|c|c|c|c|c|}
\hline nAY & $O C T$ & NOV & DEC & JAN & FER & MAR & APH & MAY & JUN & JUL & AUG & SEP \\
\hline $\begin{array}{l}1 \\
2 \\
3 \\
4 \\
5\end{array}$ & $\begin{array}{l}25 \\
26 \\
23 \\
26 \\
30\end{array}$ & $\begin{array}{l}71 \\
62 \\
53 \\
41 \\
45\end{array}$ & $\begin{array}{l}66 \\
49 \\
45 \\
49 \\
53\end{array}$ & $\begin{array}{l}62 \\
57 \\
49 \\
45 \\
41\end{array}$ & $\begin{array}{l}48 \\
58 \\
58 \\
63 \\
68\end{array}$ & $\begin{array}{l}26 \\
26 \\
26 \\
26 \\
24\end{array}$ & $\begin{array}{r}98 \\
98 \\
120 \\
220 \\
250\end{array}$ & $\begin{array}{l}330 \\
270 \\
230 \\
210 \\
180\end{array}$ & $\begin{array}{l}310 \\
270 \\
270 \\
250 \\
449\end{array}$ & $\begin{array}{lll}1 & 1 & 0 \\
1 & 1 & 0 \\
1 & 0 & 0 \\
10 & 0 \\
1 & 2 & 0\end{array}$ & $\begin{array}{l}50 \\
56 \\
62 \\
56 \\
68\end{array}$ & $\begin{array}{r}93 \\
109 \\
68 \\
100 \\
88\end{array}$ \\
\hline $\begin{array}{r}6 \\
7 \\
4 \\
9 \\
10\end{array}$ & $\begin{array}{l}30 \\
37 \\
45 \\
41 \\
33\end{array}$ & $\begin{array}{l}71 \\
66 \\
66 \\
53 \\
53\end{array}$ & $\begin{array}{l}62 \\
62 \\
57 \\
76 \\
85\end{array}$ & $\begin{array}{l}37 \\
37 \\
33 \\
30 \\
30\end{array}$ & $\begin{array}{l}60 \\
65 \\
65 \\
60 \\
60\end{array}$ & $\begin{array}{l}24 \\
22 \\
22 \\
24 \\
28\end{array}$ & $\begin{array}{r}200 \\
261 \\
1260 \\
1360 \\
708\end{array}$ & $\begin{array}{l}170 \\
140 \\
138 \\
140 \\
130\end{array}$ & $\begin{array}{r}715 \\
950 \\
1380 \\
819 \\
286\end{array}$ & $\begin{array}{lll}1 & 1 & 0 \\
1 & 1 & 0 \\
1 & 1 & 0 \\
1 & 1 & 0 \\
1 & 0 & 0\end{array}$ & $\begin{array}{r}62 \\
94 \\
738 \\
480 \\
300\end{array}$ & $\begin{array}{l}81 \\
81 \\
75 \\
94 \\
88\end{array}$ \\
\hline $\begin{array}{l}11 \\
12 \\
13 \\
14 \\
15\end{array}$ & $\begin{array}{l}37 \\
45 \\
30 \\
26 \\
30\end{array}$ & $\begin{array}{l}95 \\
45 \\
49 \\
49 \\
49\end{array}$ & $\begin{array}{l}95 \\
80 \\
57 \\
40 \\
33\end{array}$ & $\begin{array}{l}30 \\
26 \\
26 \\
23 \\
23\end{array}$ & $\begin{array}{l}53 \\
57 \\
57 \\
57 \\
57\end{array}$ & $\begin{array}{l}30 \\
28 \\
28 \\
28 \\
30\end{array}$ & $\begin{array}{l}520 \\
440 \\
380 \\
350 \\
370\end{array}$ & $\begin{array}{l}160 \\
130 \\
200 \\
290 \\
310\end{array}$ & $\begin{array}{l}222 \\
242 \\
221 \\
230 \\
260\end{array}$ & $\begin{array}{r}97 \\
97 \\
99 \\
99 \\
110\end{array}$ & $\begin{array}{r}180 \\
150 \\
120 \\
110 \\
98\end{array}$ & $\begin{array}{r}75 \\
63 \\
120 \\
130 \\
82\end{array}$ \\
\hline $\begin{array}{l}16 \\
17 \\
18 \\
19 \\
20\end{array}$ & $\begin{array}{l}311 \\
33 \\
33 \\
85 \\
49\end{array}$ & $\begin{array}{l}45 \\
53 \\
49 \\
49 \\
49\end{array}$ & $\begin{array}{l}30 \\
30 \\
30 \\
33 \\
37\end{array}$ & $\begin{array}{r}85 \\
796 \\
654 \\
262 \\
177\end{array}$ & $\begin{array}{l}45 \\
45 \\
42 \\
45 \\
60\end{array}$ & $\begin{array}{r}32 \\
244 \\
431 \\
322 \\
277\end{array}$ & $\begin{array}{l}570 \\
490 \\
430 \\
400 \\
370\end{array}$ & $\begin{array}{l}290 \\
260 \\
360 \\
400 \\
310\end{array}$ & $\begin{array}{l}200 \\
180 \\
170 \\
260 \\
310\end{array}$ & $\begin{array}{l}160 \\
140 \\
110 \\
110 \\
627\end{array}$ & $\begin{array}{r}75 \\
81 \\
88 \\
81 \\
390\end{array}$ & $\begin{array}{r}110 \\
140 \\
93 \\
68 \\
160\end{array}$ \\
\hline $\begin{array}{l}? 1 \\
? 2 \\
23 \\
24 \\
75\end{array}$ & $\begin{array}{r}53 \\
66 \\
120 \\
1411 \\
100\end{array}$ & $\begin{array}{r}76 \\
134 \\
130 \\
90 \\
76\end{array}$ & $\begin{array}{r}41 \\
49 \\
80 \\
260 \\
570\end{array}$ & $\begin{array}{r}128 \\
102 \\
90 \\
79 \\
65\end{array}$ & $\begin{array}{r}73 \\
110 \\
92 \\
73 \\
57\end{array}$ & $\begin{array}{r}200 \\
144 \\
101 \\
89 \\
115\end{array}$ & $\begin{array}{l}340 \\
320 \\
280 \\
260 \\
240\end{array}$ & $\begin{array}{l}240 \\
220 \\
190 \\
180 \\
180\end{array}$ & $\begin{array}{l}230 \\
190 \\
180 \\
160 \\
150\end{array}$ & $\begin{array}{r}474 \\
343 \\
130 \\
94 \\
81\end{array}$ & $\begin{array}{l}480 \\
310 \\
160 \\
130 \\
130\end{array}$ & $\begin{array}{r}140 \\
2250 \\
746 \\
372 \\
187\end{array}$ \\
\hline $\begin{array}{l}26 \\
27 \\
28 \\
29 \\
30 \\
31\end{array}$ & $\begin{array}{l}66 \\
66 \\
62 \\
53 \\
53 \\
49\end{array}$ & $\begin{array}{r}150 \\
240 \\
190 \\
150 \\
71 \\
-\end{array}$ & $\begin{array}{r}350 \\
160 \\
100 \\
85 \\
80 \\
66\end{array}$ & $\begin{array}{l}58 \\
51 \\
49 \\
49 \\
49 \\
43\end{array}$ & $\begin{array}{r}45 \\
45 \\
35 \\
-- \\
\hdashline--\end{array}$ & $\begin{array}{r}132 \\
120 \\
111 \\
100 \\
98 \\
98\end{array}$ & $\begin{array}{l}240 \\
230 \\
230 \\
290 \\
370 \\
---\end{array}$ & $\begin{array}{l}160 \\
150 \\
160 \\
320 \\
360 \\
370\end{array}$ & $\begin{array}{l}140 \\
120 \\
140 \\
140 \\
110 \\
-\end{array}$ & $\begin{array}{l}75 \\
75 \\
62 \\
56 \\
50 \\
62\end{array}$ & $\begin{array}{l}200 \\
180 \\
230 \\
260 \\
200 \\
150\end{array}$ & $\begin{array}{r}180 \\
140 \\
110 \\
100 \\
98 \\
-\end{array}$ \\
\hline $\begin{array}{l}\text { MAX } \\
\text { MIN }\end{array}$ & $\begin{array}{r}140 \\
23\end{array}$ & $\begin{array}{r}240 \\
41\end{array}$ & $\begin{array}{r}570 \\
30\end{array}$ & $\begin{array}{r}796 \\
23\end{array}$ & 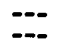 & $\begin{array}{r}431 \\
22\end{array}$ & $\begin{array}{r}1360 \\
98\end{array}$ & $\begin{array}{l}400 \\
130\end{array}$ & $\begin{array}{r}1380 \\
110\end{array}$ & $\begin{array}{r}627 \\
50\end{array}$ & $\begin{array}{r}738 \\
50\end{array}$ & $\begin{array}{r}2250 \\
63\end{array}$ \\
\hline
\end{tabular}

$\begin{array}{llllllllllll}\text { TOTAL } & 1542 & 2420 & 2910 & 3286 & 1653 & 3006 & 11696 & 7178 & 9554 & 4231 & 5769\end{array}$ TOTAL LOAD FOR YEAR: 59486 POUNDS 
Table 19. Total nitrogen load, in pounds per day, for Onion River at Hingham, 1979 and 1980 water years

TOTAL NITROGEN, WATER YEAR OCTOBER 1978 TO SEPTEMBEH 1979

\begin{tabular}{|c|c|c|c|c|c|c|c|c|c|c|c|c|}
\hline DAY & OCT & NDV & DEC & JAN & FEA & MAR & $A P K$ & MAY & JUN & JUL & AUG & SEP \\
\hline $\begin{array}{l}1 \\
2 \\
3 \\
4 \\
5\end{array}$ & $\begin{array}{l}420 \\
390 \\
360 \\
330 \\
310\end{array}$ & $\begin{array}{l}110 \\
110 \\
110 \\
110 \\
110\end{array}$ & $\begin{array}{l}270 \\
260 \\
250 \\
230 \\
220\end{array}$ & $\begin{array}{l}130 \\
130 \\
130 \\
120 \\
120\end{array}$ & $\begin{array}{l}100 \\
100 \\
100 \\
100 \\
100\end{array}$ & $\begin{array}{l}170 \\
180 \\
190 \\
200 \\
210\end{array}$ & $\begin{array}{l}2590 \\
1770 \\
1460 \\
1850 \\
2190\end{array}$ & $\begin{array}{r}1130 \\
990 \\
1750 \\
1400 \\
880\end{array}$ & $\begin{array}{l}320 \\
270 \\
240 \\
320 \\
300\end{array}$ & $\begin{array}{l}673 \\
470 \\
260 \\
280 \\
280\end{array}$ & $\begin{array}{l}160 \\
140 \\
130 \\
130 \\
220\end{array}$ & $\begin{array}{l}190 \\
190 \\
150 \\
140 \\
140\end{array}$ \\
\hline $\begin{array}{r}6 \\
7 \\
8 \\
9 \\
10\end{array}$ & $\begin{array}{l}460 \\
4 n 0 \\
360 \\
340 \\
310\end{array}$ & $\begin{array}{l}110 \\
120 \\
120 \\
120 \\
130\end{array}$ & $\begin{array}{l}220 \\
220 \\
220 \\
210 \\
190\end{array}$ & $\begin{array}{l}120 \\
120 \\
120 \\
120 \\
120\end{array}$ & 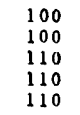 & $\begin{array}{l}220 \\
220 \\
220 \\
210 \\
210\end{array}$ & $\begin{array}{r}1810 \\
1110 \\
900 \\
820 \\
820\end{array}$ & $\begin{array}{l}730 \\
640 \\
570 \\
520 \\
470\end{array}$ & $\begin{array}{l}280 \\
370 \\
300 \\
350 \\
590\end{array}$ & $\begin{array}{l}200 \\
170 \\
140 \\
140 \\
170\end{array}$ & $\begin{array}{r}190 \\
1200 \\
81 \\
463 \\
2720\end{array}$ & $\begin{array}{l}150 \\
120 \\
120 \\
120 \\
120\end{array}$ \\
\hline $\begin{array}{l}11 \\
12 \\
13 \\
14 \\
15\end{array}$ & $\begin{array}{l}290 \\
270 \\
240 \\
240 \\
230\end{array}$ & $\begin{array}{l}130 \\
120 \\
130 \\
150 \\
180\end{array}$ & $\begin{array}{l}190 \\
180 \\
180 \\
180 \\
180\end{array}$ & $\begin{array}{l}120 \\
120 \\
110 \\
110 \\
110\end{array}$ & 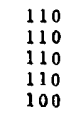 & $\begin{array}{l}200 \\
200 \\
200 \\
210 \\
220\end{array}$ & $\begin{array}{r}840 \\
3780 \\
3000 \\
1650 \\
1110\end{array}$ & $\begin{array}{l}450 \\
420 \\
400 \\
450 \\
430\end{array}$ & $\begin{array}{l}430 \\
330 \\
280 \\
250 \\
240\end{array}$ & $\begin{array}{l}230 \\
180 \\
300 \\
230 \\
170\end{array}$ & $\begin{array}{r}1340 \\
559 \\
363 \\
290 \\
217\end{array}$ & $\begin{array}{l}120 \\
130 \\
130 \\
120 \\
110\end{array}$ \\
\hline $\begin{array}{l}16 \\
17 \\
18 \\
19 \\
20\end{array}$ & $\begin{array}{l}240 \\
260 \\
240 \\
230 \\
230\end{array}$ & $\begin{array}{l}180 \\
290 \\
580 \\
500 \\
420\end{array}$ & $\begin{array}{l}170 \\
170 \\
170 \\
170 \\
170\end{array}$ & $\begin{array}{l}110 \\
110 \\
120 \\
130 \\
130\end{array}$ & $\begin{array}{l}100 \\
100 \\
110 \\
110 \\
120\end{array}$ & $\begin{array}{r}210 \\
210 \\
289 \\
1120 \\
4170\end{array}$ & $\begin{array}{l}880 \\
750 \\
680 \\
606 \\
570\end{array}$ & $\begin{array}{l}370 \\
380 \\
420 \\
520 \\
520\end{array}$ & $\begin{array}{l}220 \\
220 \\
210 \\
210 \\
350\end{array}$ & $\begin{array}{l}130 \\
120 \\
110 \\
110 \\
110\end{array}$ & $\begin{array}{l}186 \\
184 \\
183 \\
178 \\
541\end{array}$ & $\begin{array}{r}110 \\
110 \\
110 \\
90 \\
94\end{array}$ \\
\hline $\begin{array}{l}21 \\
22 \\
23 \\
24 \\
25\end{array}$ & $\begin{array}{l}220 \\
180 \\
130 \\
150 \\
170\end{array}$ & $\begin{array}{l}370 \\
360 \\
430 \\
440 \\
430\end{array}$ & $\begin{array}{l}170 \\
150 \\
150 \\
150 \\
150\end{array}$ & $\begin{array}{l}130 \\
130 \\
120 \\
120 \\
120\end{array}$ & $\begin{array}{l}120 \\
120 \\
130 \\
130 \\
130\end{array}$ & $\begin{array}{l}5820 \\
6050 \\
7470 \\
7250 \\
3200\end{array}$ & $\begin{array}{l}550 \\
520 \\
501 \\
480 \\
750\end{array}$ & $\begin{array}{l}500 \\
420 \\
400 \\
400 \\
337\end{array}$ & $\begin{array}{l}330 \\
270 \\
220 \\
180 \\
180\end{array}$ & $\begin{array}{r}110 \\
96 \\
110 \\
110 \\
230\end{array}$ & $\begin{array}{l}650 \\
430 \\
660 \\
610 \\
420\end{array}$ & $\begin{array}{r}110 \\
94 \\
94 \\
110 \\
110\end{array}$ \\
\hline $\begin{array}{l}26 \\
27 \\
28 \\
29 \\
30 \\
31\end{array}$ & $\begin{array}{r}150 \\
140 \\
120 \\
110 \\
90 \\
94\end{array}$ & $\begin{array}{l}400 \\
360 \\
330 \\
300 \\
290 \\
\cdots\end{array}$ & $\begin{array}{l}140 \\
140 \\
140 \\
140 \\
140 \\
140\end{array}$ & $\begin{array}{l}120 \\
120 \\
110 \\
110 \\
100 \\
100\end{array}$ & \begin{tabular}{l}
140 \\
150 \\
165 \\
\hdashline-2 \\
$\ldots-$
\end{tabular} & $\begin{array}{l}1940 \\
1700 \\
1730 \\
1730 \\
8110 \\
6390\end{array}$ & $\begin{array}{r}1850 \\
1590 \\
940 \\
730 \\
1070 \\
\end{array}$ & $\begin{array}{l}320 \\
280 \\
280 \\
280 \\
280 \\
420\end{array}$ & $\begin{array}{l}160 \\
160 \\
164 \\
685 \\
703 \\
-\end{array}$ & $\begin{array}{l}190 \\
160 \\
140 \\
120 \\
160 \\
210\end{array}$ & $\begin{array}{l}300 \\
290 \\
270 \\
270 \\
260 \\
210\end{array}$ & $\begin{array}{r}110 \\
94 \\
94 \\
94 \\
110 \\
\end{array}$ \\
\hline ME AN & 249 & 251 & 183 & 119 & 114 & 1950 & 1272 & 560 & 304 & 197 & 447 & 119 \\
\hline WTR YR & & MEAN & 483 & $\max$ & 8110 & & & 81 & & & & \\
\hline TOTAL & 7704 & 7540 & 5660 & 3680 & 3195 & 60449 & 38167 & 17357 & 9132 & 6109 & 13845 & 3584 \\
\hline
\end{tabular}

TOTAL LOAD FOR YEAR: 176422 POUNOS

TOTAL NITROGEN, WATER YEAR OCTOGER 1979 TO SEPTEMBEH 1980

\begin{tabular}{|c|c|c|c|c|c|c|c|c|c|c|c|c|}
\hline DAY & $0 \mathrm{CT}$ & Nov & DEC & JAN & FEB & MAK & APR & MAY & JUN & JUL & AUG & SEP \\
\hline $\begin{array}{l}1 \\
2 \\
3 \\
4 \\
5\end{array}$ & $\begin{array}{r}97 \\
120 \\
110 \\
120 \\
120\end{array}$ & $\begin{array}{l}260 \\
230 \\
210 \\
170 \\
140\end{array}$ & $\begin{array}{l}240 \\
190 \\
180 \\
190 \\
210\end{array}$ & $\begin{array}{l}230 \\
220 \\
190 \\
180 \\
170\end{array}$ & $\begin{array}{l}240 \\
260 \\
260 \\
280 \\
300\end{array}$ & $\begin{array}{l}130 \\
130 \\
130 \\
130 \\
120\end{array}$ & $\begin{array}{l}390 \\
390 \\
460 \\
730 \\
810\end{array}$ & $\begin{array}{l}510 \\
400 \\
330 \\
290 \\
250\end{array}$ & $\begin{array}{l}480 \\
400 \\
400 \\
360 \\
560\end{array}$ & $\begin{array}{r}110 \\
110 \\
100 \\
96 \\
120\end{array}$ & $\begin{array}{l}110 \\
120 \\
140 \\
120 \\
150\end{array}$ & $\begin{array}{l}310 \\
350 \\
240 \\
210 \\
190\end{array}$ \\
\hline $\begin{array}{r}6 \\
7 \\
8 \\
9 \\
10\end{array}$ & $\begin{array}{l}130 \\
160 \\
180 \\
170 \\
140\end{array}$ & $\begin{array}{l}260 \\
240 \\
240 \\
210 \\
210\end{array}$ & $\begin{array}{l}230 \\
230 \\
220 \\
270 \\
300\end{array}$ & $\begin{array}{l}160 \\
160 \\
140 \\
130 \\
130\end{array}$ & $\begin{array}{l}230 \\
240 \\
240 \\
230 \\
230\end{array}$ & $\begin{array}{l}120 \\
110 \\
110 \\
120 \\
140\end{array}$ & $\begin{array}{r}670 \\
928 \\
3540 \\
4190 \\
2710\end{array}$ & $\begin{array}{l}220 \\
170 \\
202 \\
170 \\
160\end{array}$ & $\begin{array}{r}1320 \\
1810 \\
2480 \\
1400 \\
460\end{array}$ & $\begin{array}{l}1110 \\
1100 \\
110 \\
110 \\
96\end{array}$ & $\begin{array}{r}140 \\
200 \\
1280 \\
954 \\
578\end{array}$ & $\begin{array}{l}170 \\
198 \\
160 \\
200 \\
190\end{array}$ \\
\hline $\begin{array}{l}11 \\
12 \\
13 \\
14 \\
15\end{array}$ & $\begin{array}{l}160 \\
180 \\
130 \\
120 \\
130\end{array}$ & $\begin{array}{l}330 \\
180 \\
190 \\
190 \\
190\end{array}$ & $\begin{array}{l}330 \\
280 \\
220 \\
166 \\
140\end{array}$ & $\begin{array}{l}130 \\
120 \\
120 \\
110 \\
110\end{array}$ & $\begin{array}{l}150 \\
160 \\
160 \\
160 \\
160\end{array}$ & $\begin{array}{l}150 \\
140 \\
140 \\
140 \\
150\end{array}$ & $\begin{array}{r}1110 \\
900 \\
780 \\
700 \\
750\end{array}$ & $\begin{array}{l}210 \\
160 \\
280 \\
440 \\
480\end{array}$ & $\begin{array}{l}300 \\
260 \\
240 \\
290 \\
330\end{array}$ & $\begin{array}{r}90 \\
90 \\
93 \\
93 \\
110\end{array}$ & $\begin{array}{l}356 \\
315 \\
264 \\
245 \\
211\end{array}$ & $\begin{array}{l}160 \\
200 \\
340 \\
360 \\
260\end{array}$ \\
\hline $\begin{array}{l}16 \\
17 \\
18 \\
19 \\
20\end{array}$ & $\begin{array}{l}130 \\
140 \\
140 \\
300 \\
190\end{array}$ & $\begin{array}{l}205 \\
210 \\
190 \\
190 \\
190\end{array}$ & $\begin{array}{l}130 \\
130 \\
130 \\
140 \\
160\end{array}$ & $\begin{array}{r}230 \\
1500 \\
1260 \\
560 \\
420\end{array}$ & $\begin{array}{l}150 \\
150 \\
140 \\
150 \\
170\end{array}$ & $\begin{array}{l}160 \\
540 \\
910 \\
672 \\
630\end{array}$ & $\begin{array}{r}1010 \\
850 \\
710 \\
660 \\
590\end{array}$ & $\begin{array}{l}440 \\
380 \\
580 \\
660 \\
470\end{array}$ & $\begin{array}{l}240 \\
210 \\
190 \\
340 \\
420\end{array}$ & $\begin{array}{r}170 \\
150 \\
110 \\
110 \\
1110\end{array}$ & $\begin{array}{l}160 \\
170 \\
190 \\
170 \\
760\end{array}$ & $\begin{array}{l}310 \\
400 \\
280 \\
220 \\
410\end{array}$ \\
\hline $\begin{array}{l}21 \\
22 \\
23 \\
24 \\
25\end{array}$ & $\begin{array}{l}210 \\
240 \\
380 \\
450 \\
350\end{array}$ & $\begin{array}{l}270 \\
420 \\
410 \\
310 \\
270\end{array}$ & $\begin{array}{r}170 \\
190 \\
280 \\
710 \\
1320\end{array}$ & $\begin{array}{l}338 \\
300 \\
290 \\
280 \\
250\end{array}$ & $\begin{array}{l}180 \\
230 \\
200 \\
180 \\
160\end{array}$ & $\begin{array}{l}493 \\
388 \\
297 \\
286 \\
399\end{array}$ & $\begin{array}{l}540 \\
500 \\
420 \\
380 \\
350\end{array}$ & $\begin{array}{l}350 \\
310 \\
260 \\
240 \\
240\end{array}$ & $\begin{array}{l}290 \\
220 \\
200 \\
174 \\
160\end{array}$ & $\begin{array}{l}983 \\
603 \\
270 \\
200 \\
170\end{array}$ & $\begin{array}{l}920 \\
610 \\
320 \\
270 \\
270\end{array}$ & $\begin{array}{r}390 \\
4035 \\
1640 \\
784 \\
485\end{array}$ \\
\hline $\begin{array}{l}26 \\
27 \\
28 \\
29 \\
30 \\
31\end{array}$ & $\begin{array}{l}240 \\
240 \\
230 \\
210 \\
210 \\
190\end{array}$ & $\begin{array}{l}470 \\
680 \\
560 \\
470 \\
260 \\
\ldots\end{array}$ & $\begin{array}{l}900 \\
480 \\
340 \\
300 \\
280 \\
240\end{array}$ & $\begin{array}{l}240 \\
230 \\
230 \\
210 \\
210 \\
240\end{array}$ & $\begin{array}{l}150 \\
150 \\
140 \\
140 \\
\ldots \ldots\end{array}$ & $\begin{array}{l}499 \\
493 \\
460 \\
400 \\
390 \\
390\end{array}$ & $\begin{array}{l}350 \\
330 \\
330 \\
430 \\
590 \\
\ldots-\end{array}$ & $\begin{array}{l}210 \\
200 \\
210 \\
500 \\
560 \\
580\end{array}$ & $\begin{array}{l}150 \\
130 \\
150 \\
150 \\
110 \\
1\end{array}$ & $\begin{array}{l}160 \\
160 \\
240 \\
120 \\
110 \\
140\end{array}$ & $\begin{array}{l}406 \\
360 \\
460 \\
520 \\
410 \\
310\end{array}$ & $\begin{array}{l}460 \\
390 \\
330 \\
300 \\
290 \\
-\end{array}$ \\
\hline MFAN & 191 & 280 & 300 & 293 & 193 & 302 & 903 & 337 & 474 & 205 & 371 & 475 \\
\hline WTR YR & & MEAN & 359 & $\max$ & 4190 & & & 90 & & & & \\
\hline TOTAL & 5917 & 7985 & 9296 & 9088 & 5590 & 9367 & 27098 & 10462 & 14224 & 6354 & 11489 & 14268 \\
\hline
\end{tabular}

TOTAL LOAD FOR YEAR: 131138 POUNDS 
Table 20. Total nitrite plus nitrate nitrogen load, in pounds per day, for Onion River at Hingham, 1979 and 1980 water years

TOTAL NITRITE PLUS NITRATE, WATER YEAH OCTOBER 1978 TO SEPTEMBER 1979

\begin{tabular}{|c|c|c|c|c|c|c|c|c|c|c|c|c|}
\hline nAY & OCT & inov & DEC & JAN & FEA & MAR & APN & MAY & JUN & JUL & AUG & SEP \\
\hline $\begin{array}{l}1 \\
2 \\
3 \\
4 \\
5\end{array}$ & $\begin{array}{l}310 \\
290 \\
280 \\
260 \\
250\end{array}$ & $\begin{array}{l}100 \\
100 \\
100 \\
100 \\
100\end{array}$ & $\begin{array}{l}220 \\
210 \\
200 \\
190 \\
180\end{array}$ & $\begin{array}{l}130 \\
130 \\
130 \\
120 \\
120\end{array}$ & $\begin{array}{l}85 \\
85 \\
85 \\
85 \\
85\end{array}$ & $\begin{array}{l}120 \\
130 \\
140 \\
140 \\
150\end{array}$ & $\begin{array}{r}1820 \\
940 \\
760 \\
990 \\
1200\end{array}$ & $\begin{array}{l}560 \\
480 \\
930 \\
720 \\
420\end{array}$ & $\begin{array}{r}130 \\
110 \\
94 \\
130 \\
120\end{array}$ & $\begin{array}{r}329 \\
250 \\
74 \\
78 \\
78\end{array}$ & $\begin{array}{l}39 \\
35 \\
32 \\
32 \\
60\end{array}$ & $\begin{array}{l}98 \\
98 \\
81 \\
75 \\
75\end{array}$ \\
\hline $\begin{array}{r}6 \\
7 \\
8 \\
9 \\
10\end{array}$ & $\begin{array}{l}340 \\
300 \\
280 \\
270 \\
250\end{array}$ & $\begin{array}{l}100 \\
110 \\
110 \\
110 \\
120\end{array}$ & $\begin{array}{l}205 \\
200 \\
200 \\
190 \\
180\end{array}$ & $\begin{array}{l}120 \\
120 \\
120 \\
120 \\
120\end{array}$ & $\begin{array}{l}85 \\
85 \\
92 \\
92 \\
92\end{array}$ & $\begin{array}{l}160 \\
160 \\
160 \\
150 \\
150\end{array}$ & $\begin{array}{l}960 \\
550 \\
430 \\
390 \\
390\end{array}$ & $\begin{array}{l}340 \\
290 \\
260 \\
230 \\
200\end{array}$ & $\begin{array}{l}120 \\
150 \\
120 \\
150 \\
270\end{array}$ & $\begin{array}{l}55 \\
43 \\
35 \\
35 \\
43\end{array}$ & $\begin{array}{r}51 \\
39 \\
28 \\
74 \\
958\end{array}$ & $\begin{array}{l}81 \\
64 \\
64 \\
64 \\
64\end{array}$ \\
\hline $\begin{array}{l}11 \\
12 \\
13 \\
14 \\
15\end{array}$ & $\begin{array}{l}230 \\
220 \\
200 \\
200 \\
190\end{array}$ & $\begin{array}{l}120 \\
110 \\
120 \\
140 \\
160\end{array}$ & $\begin{array}{l}180 \\
170 \\
170 \\
170 \\
170\end{array}$ & $\begin{array}{lll}1 & 1 & 0 \\
1 & 1 & 0 \\
1 & 0 & 0 \\
1 & 0 & 0 \\
1 & 0 & 0\end{array}$ & $\begin{array}{l}84 \\
84 \\
84 \\
94 \\
77\end{array}$ & $\begin{array}{l}140 \\
140 \\
140 \\
150 \\
160\end{array}$ & $\begin{array}{r}400 \\
2240 \\
1720 \\
860 \\
550\end{array}$ & $\begin{array}{l}200 \\
180 \\
170 \\
200 \\
190\end{array}$ & $\begin{array}{r}190 \\
140 \\
120 \\
100 \\
94\end{array}$ & $\begin{array}{l}64 \\
47 \\
88 \\
64 \\
43\end{array}$ & $\begin{array}{r}540 \\
183 \\
101 \\
71 \\
46\end{array}$ & $\begin{array}{l}64 \\
69 \\
69 \\
64 \\
58\end{array}$ \\
\hline $\begin{array}{l}16 \\
17 \\
18 \\
19 \\
? 0\end{array}$ & $\begin{array}{l}200 \\
210 \\
200 \\
190 \\
190\end{array}$ & $\begin{array}{l}160 \\
230 \\
410 \\
370 \\
310\end{array}$ & $\begin{array}{l}160 \\
160 \\
160 \\
160 \\
160\end{array}$ & $\begin{array}{l}100 \\
100 \\
110 \\
120 \\
120\end{array}$ & $\begin{array}{l}77 \\
77 \\
84 \\
84 \\
91\end{array}$ & $\begin{array}{r}150 \\
150 \\
190 \\
780 \\
3050\end{array}$ & $\begin{array}{l}420 \\
350 \\
310 \\
280 \\
260\end{array}$ & $\begin{array}{l}150 \\
160 \\
180 \\
230 \\
230\end{array}$ & $\begin{array}{r}88 \\
88 \\
81 \\
81 \\
150\end{array}$ & $\begin{array}{l}32 \\
28 \\
23 \\
23 \\
23\end{array}$ & $\begin{array}{r}35 \\
30 \\
26 \\
22 \\
172\end{array}$ & $\begin{array}{l}58 \\
58 \\
58 \\
50 \\
52\end{array}$ \\
\hline $\begin{array}{l}21 \\
22 \\
23 \\
24 \\
25\end{array}$ & $\begin{array}{l}180 \\
160 \\
120 \\
140 \\
150\end{array}$ & $\begin{array}{l}280 \\
280 \\
320 \\
360 \\
320\end{array}$ & $\begin{array}{l}160 \\
140 \\
140 \\
140 \\
140\end{array}$ & $\begin{array}{l}110 \\
110 \\
100 \\
100 \\
100\end{array}$ & $\begin{array}{l}84 \\
84 \\
91 \\
91 \\
91\end{array}$ & $\begin{array}{l}4280 \\
4540 \\
5650 \\
5340 \\
2330\end{array}$ & $\begin{array}{l}250 \\
230 \\
220 \\
210 \\
350\end{array}$ & $\begin{array}{l}220 \\
180 \\
170 \\
170 \\
175\end{array}$ & $\begin{array}{r}140 \\
110 \\
88 \\
68 \\
68\end{array}$ & $\begin{array}{l}23 \\
22 \\
23 \\
23 \\
64\end{array}$ & $\begin{array}{l}290 \\
200 \\
290 \\
270 \\
190\end{array}$ & $\begin{array}{l}58 \\
52 \\
52 \\
58 \\
58\end{array}$ \\
\hline $\begin{array}{l}26 \\
27 \\
28 \\
29 \\
30 \\
31\end{array}$ & $\begin{array}{r}140 \\
130 \\
110 \\
100 \\
88 \\
92\end{array}$ & $\begin{array}{l}300 \\
280 \\
260 \\
240 \\
230 \\
---\end{array}$ & $\begin{array}{l}140 \\
140 \\
140 \\
140 \\
140 \\
140\end{array}$ & $\begin{array}{r}100 \\
100 \\
97 \\
97 \\
89 \\
89\end{array}$ & \begin{tabular}{r}
97 \\
110 \\
117 \\
-2 \\
\hdashline- \\
-
\end{tabular} & $\begin{array}{l}1400 \\
1210 \\
1110 \\
1200 \\
4520 \\
3980\end{array}$ & $\begin{array}{l}990 \\
830 \\
450 \\
340 \\
530 \\
---\end{array}$ & $\begin{array}{l}130 \\
110 \\
110 \\
110 \\
110 \\
180\end{array}$ & $\begin{array}{r}61 \\
61 \\
42 \\
266 \\
379 \\
-\end{array}$ & $\begin{array}{l}51 \\
39 \\
35 \\
28 \\
39 \\
55\end{array}$ & $\begin{array}{l}140 \\
140 \\
130 \\
130 \\
130 \\
100\end{array}$ & $\begin{array}{r}58 \\
70 \\
70 \\
70 \\
80 \\
-\end{array}$ \\
\hline ME AN & 202 & 202 & 168 & 109 & $8 \mathrm{~B}$ & 1357 & 674 & 258 & 127 & 60 & 148 & 66 \\
\hline WTR YR & & MEAN & 290 & MAX & 5650 & & & & & & & \\
\hline TOTAL & 6270 & 6050 & 5195 & 3392 & 2452 & 42070 & 20220 & 7825 & 3809 & 1857 & 4584 & 1990 \\
\hline
\end{tabular}

TOTAL LOAD FOR YEAR: 105714 POUNDS

TOTAL NITHITE PLUS NITRATE, WATER YEAR OCTOBEK 1979 TO SEPTEMBER 1980

\begin{tabular}{|c|c|c|c|c|c|c|c|c|c|c|c|c|}
\hline DAY & $U C T$ & NOV & $\mathrm{DEC}$ & JAN & FEB & MAH & APR & MAY & JUN & JUL & AUG & SEP \\
\hline $\begin{array}{l}1 \\
2 \\
3 \\
4 \\
5\end{array}$ & $\begin{array}{l}74 \\
93 \\
86 \\
93 \\
93\end{array}$ & $\begin{array}{l}170 \\
160 \\
140 \\
120 \\
130\end{array}$ & $\begin{array}{l}160 \\
140 \\
130 \\
140 \\
140\end{array}$ & $\begin{array}{l}160 \\
150 \\
140 \\
130 \\
120\end{array}$ & $\begin{array}{r}91 \\
100 \\
100 \\
110 \\
120\end{array}$ & $\begin{array}{r}100 \\
100 \\
100 \\
100 \\
91\end{array}$ & $\begin{array}{l}300 \\
300 \\
350 \\
570 \\
640\end{array}$ & $\begin{array}{r}200 \\
140 \\
98 \\
80 \\
64\end{array}$ & $\begin{array}{r}140 \\
110 \\
110 \\
98 \\
109\end{array}$ & $\begin{array}{l}22 \\
22 \\
19 \\
18 \\
25\end{array}$ & $\begin{array}{l}22 \\
25 \\
28 \\
25 \\
31\end{array}$ & $\begin{array}{l}160 \\
180 \\
130 \\
120 \\
100\end{array}$ \\
\hline $\begin{array}{r}6 \\
7 \\
8 \\
9 \\
10\end{array}$ & $\begin{array}{l}100 \\
110 \\
130 \\
120 \\
110\end{array}$ & $\begin{array}{l}170 \\
160 \\
160 \\
140 \\
140\end{array}$ & $\begin{array}{l}160 \\
160 \\
150 \\
180 \\
190\end{array}$ & $\begin{array}{l}110 \\
110 \\
110 \\
100 \\
100\end{array}$ & $\begin{array}{l}120 \\
130 \\
130 \\
120 \\
120\end{array}$ & $\begin{array}{r}91 \\
82 \\
82 \\
91 \\
110\end{array}$ & $\begin{array}{r}530 \\
584 \\
2120 \\
2710 \\
1900\end{array}$ & $\begin{array}{l}54 \\
36 \\
38 \\
38 \\
35\end{array}$ & $\begin{array}{l}571 \\
848 \\
978 \\
514 \\
172\end{array}$ & $\begin{array}{l}22 \\
21 \\
21 \\
21 \\
18\end{array}$ & $\begin{array}{r}25 \\
110 \\
556 \\
437 \\
293\end{array}$ & $\begin{array}{r}97 \\
132 \\
90 \\
110 \\
100\end{array}$ \\
\hline $\begin{array}{l}11 \\
12 \\
13 \\
14 \\
15\end{array}$ & $\begin{array}{r}110 \\
130 \\
100 \\
93 \\
100\end{array}$ & $\begin{array}{l}210 \\
130 \\
140 \\
140 \\
140\end{array}$ & $\begin{array}{l}210 \\
190 \\
150 \\
120 \\
110\end{array}$ & $\begin{array}{r}100 \\
93 \\
93 \\
86 \\
86\end{array}$ & $\begin{array}{l}120 \\
130 \\
130 \\
130 \\
130\end{array}$ & $\begin{array}{l}120 \\
110 \\
110 \\
110 \\
120\end{array}$ & $\begin{array}{l}680 \\
490 \\
390 \\
320 \\
360\end{array}$ & $\begin{array}{r}50 \\
35 \\
68 \\
120 \\
140\end{array}$ & $\begin{array}{l}76 \\
64 \\
56 \\
72 \\
85\end{array}$ & $\begin{array}{l}16 \\
16 \\
17 \\
17 \\
22\end{array}$ & $\begin{array}{l}186 \\
164 \\
138 \\
128 \\
110\end{array}$ & $\begin{array}{r}90 \\
120 \\
200 \\
210 \\
150\end{array}$ \\
\hline $\begin{array}{l}16 \\
17 \\
18 \\
19 \\
20\end{array}$ & $\begin{array}{l}100 \\
110 \\
110 \\
190 \\
140\end{array}$ & $\begin{array}{l}154 \\
140 \\
140 \\
140 \\
140\end{array}$ & $\begin{array}{l}100 \\
100 \\
100 \\
110 \\
110\end{array}$ & $\begin{array}{l}140 \\
640 \\
490 \\
220 \\
180\end{array}$ & $\begin{array}{l}120 \\
120 \\
110 \\
120 \\
140\end{array}$ & $\begin{array}{l}130 \\
150 \\
380 \\
565 \\
518\end{array}$ & $\begin{array}{l}580 \\
440 \\
340 \\
300 \\
250\end{array}$ & $\begin{array}{l}120 \\
100 \\
180 \\
210 \\
140\end{array}$ & $\begin{array}{r}56 \\
49 \\
42 \\
89 \\
120\end{array}$ & $\begin{array}{r}38 \\
31 \\
22 \\
22 \\
369\end{array}$ & $\begin{array}{r}90 \\
97 \\
100 \\
97 \\
370\end{array}$ & $\begin{array}{l}180 \\
220 \\
160 \\
130 \\
240\end{array}$ \\
\hline $\begin{array}{l}21 \\
22 \\
23 \\
24 \\
25\end{array}$ & $\begin{array}{l}140 \\
170 \\
240 \\
270 \\
220\end{array}$ & $\begin{array}{l}180 \\
260 \\
250 \\
200 \\
190\end{array}$ & $\begin{array}{l}120 \\
140 \\
190 \\
390 \\
630\end{array}$ & $\begin{array}{l}163 \\
160 \\
170 \\
180 \\
120\end{array}$ & $\begin{array}{l}150 \\
190 \\
170 \\
150 \\
130\end{array}$ & $\begin{array}{l}397 \\
306 \\
229 \\
216 \\
295\end{array}$ & $\begin{array}{l}210 \\
190 \\
140 \\
120 \\
110\end{array}$ & $\begin{array}{l}94 \\
81 \\
64 \\
56 \\
56\end{array}$ & $\begin{array}{l}72 \\
53 \\
45 \\
38 \\
35\end{array}$ & $\begin{array}{r}459 \\
235 \\
64 \\
45 \\
38\end{array}$ & $\begin{array}{l}450 \\
300 \\
170 \\
140 \\
140\end{array}$ & $\begin{array}{r}220 \\
1570 \\
873 \\
387 \\
276\end{array}$ \\
\hline $\begin{array}{l}26 \\
27 \\
28 \\
29 \\
30 \\
31\end{array}$ & $\begin{array}{l}160 \\
160 \\
160 \\
140 \\
140 \\
140\end{array}$ & $\begin{array}{l}280 \\
370 \\
320 \\
280 \\
170 \\
---\end{array}$ & $\begin{array}{l}470 \\
280 \\
210 \\
190 \\
190 \\
160\end{array}$ & $\begin{array}{l}80 \\
76 \\
74 \\
73 \\
73 \\
82\end{array}$ & $\begin{array}{l}120 \\
120 \\
110 \\
110 \\
-0- \\
-\cdots\end{array}$ & $\begin{array}{l}361 \\
350 \\
326 \\
310 \\
300 \\
300\end{array}$ & $\begin{array}{r}110 \\
98 \\
98 \\
150 \\
250 \\
---\end{array}$ & $\begin{array}{r}50 \\
45 \\
49 \\
140 \\
170 \\
180\end{array}$ & $\begin{array}{r}31 \\
25 \\
31 \\
31 \\
21 \\
-\end{array}$ & $\begin{array}{l}35 \\
35 \\
28 \\
25 \\
22 \\
28\end{array}$ & $\begin{array}{l}210 \\
190 \\
240 \\
260 \\
210 \\
160\end{array}$ & $\begin{array}{l}260 \\
220 \\
190 \\
180 \\
170 \\
-\end{array}$ \\
\hline MEAN & 133 & 182 & 180 & 149 & 126 & 215 & 521 & 95 & 158 & 58 & 177 & 242 \\
\hline WTR YR & & MEAN & 186 & $\max$ & 2710 & & & 16 & & & & \\
\hline TOTAL & 4136 & 5454 & 5820 & 4609 & 3641 & 6650 & 15630 & 2931 & 4741 & 1796 & 5502 & 7265 \\
\hline
\end{tabular}


Table 21. Total ammonia nitrogen load, in pounds per day, for Onion River at Hingham, 1979 and 1980 water years

TOTAL AMMONIA NITROGEN, WATER YEAR OCTOBER 1978 TO SEPTEMBER 1979

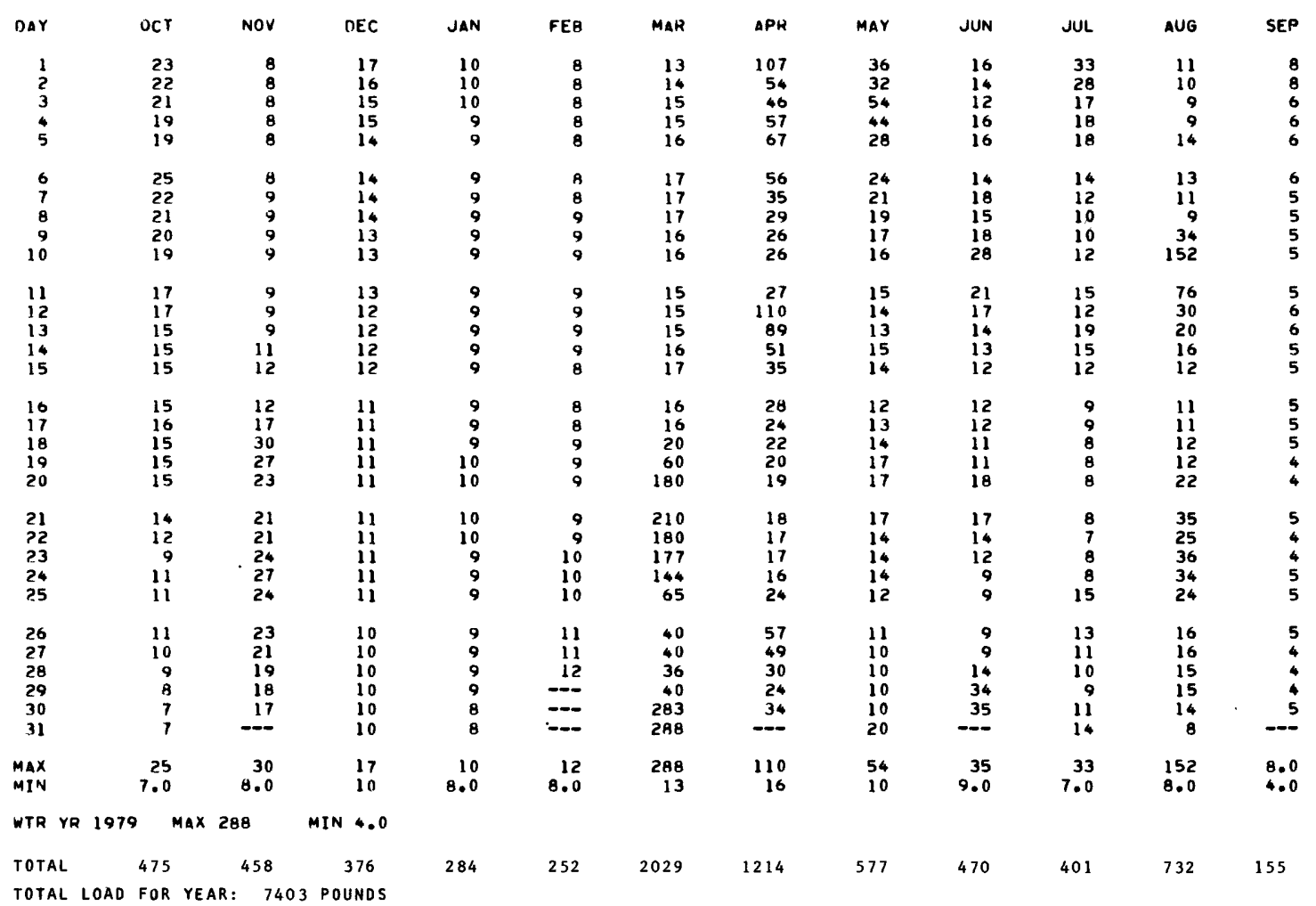

TOTAL AMMONIA NITROGEN, WATER YEAR OCTOBER 1979 TO SEPTEMBER 1980

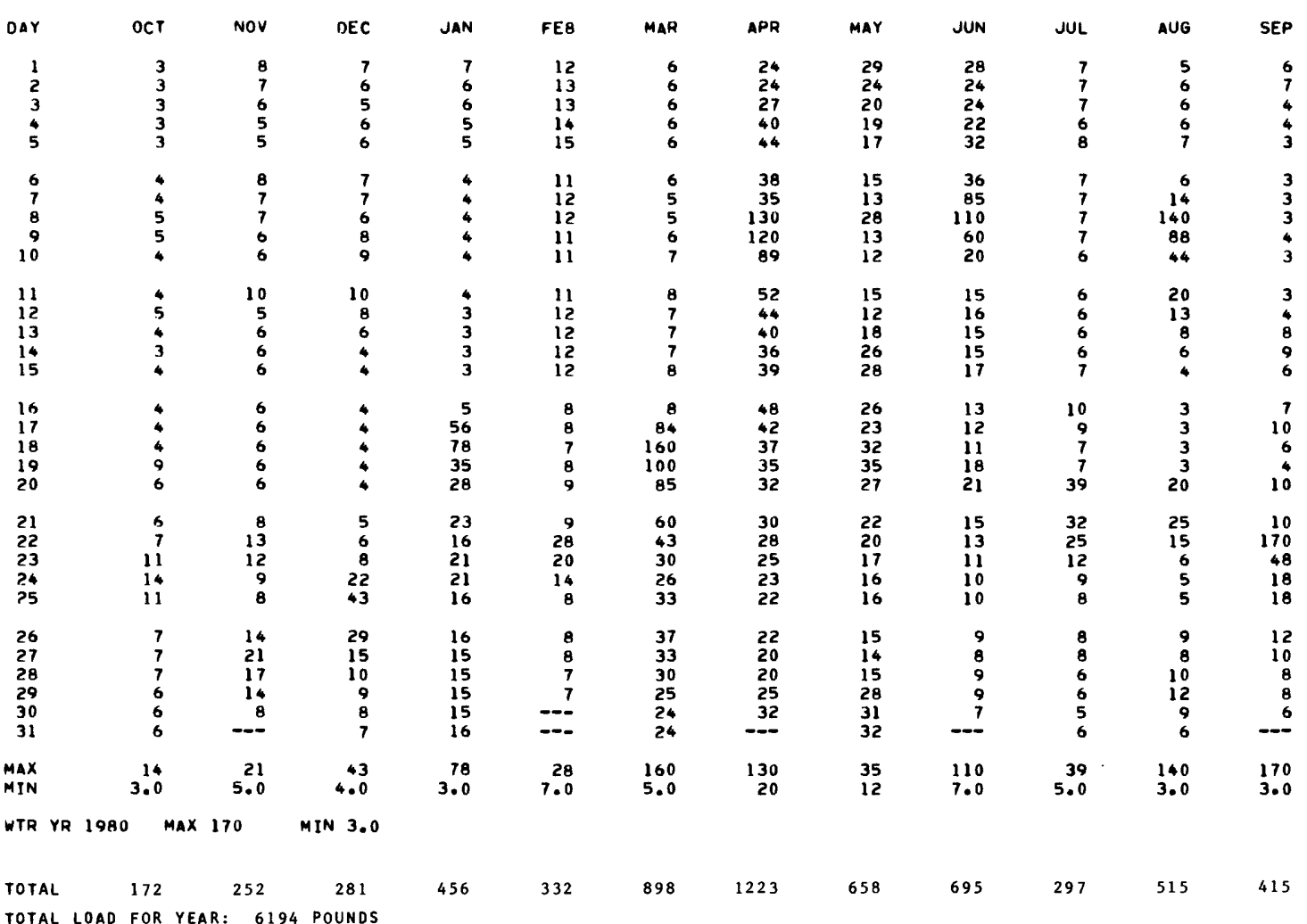


Table 22. Total phosphorus load, in pounds per day, for Onion River at Hingham, 1979 and 1980 water years

TOTAL PHOSPHORUS, WATEK YF.AR OCTORER 1978 TO SEPTEMBER 1979

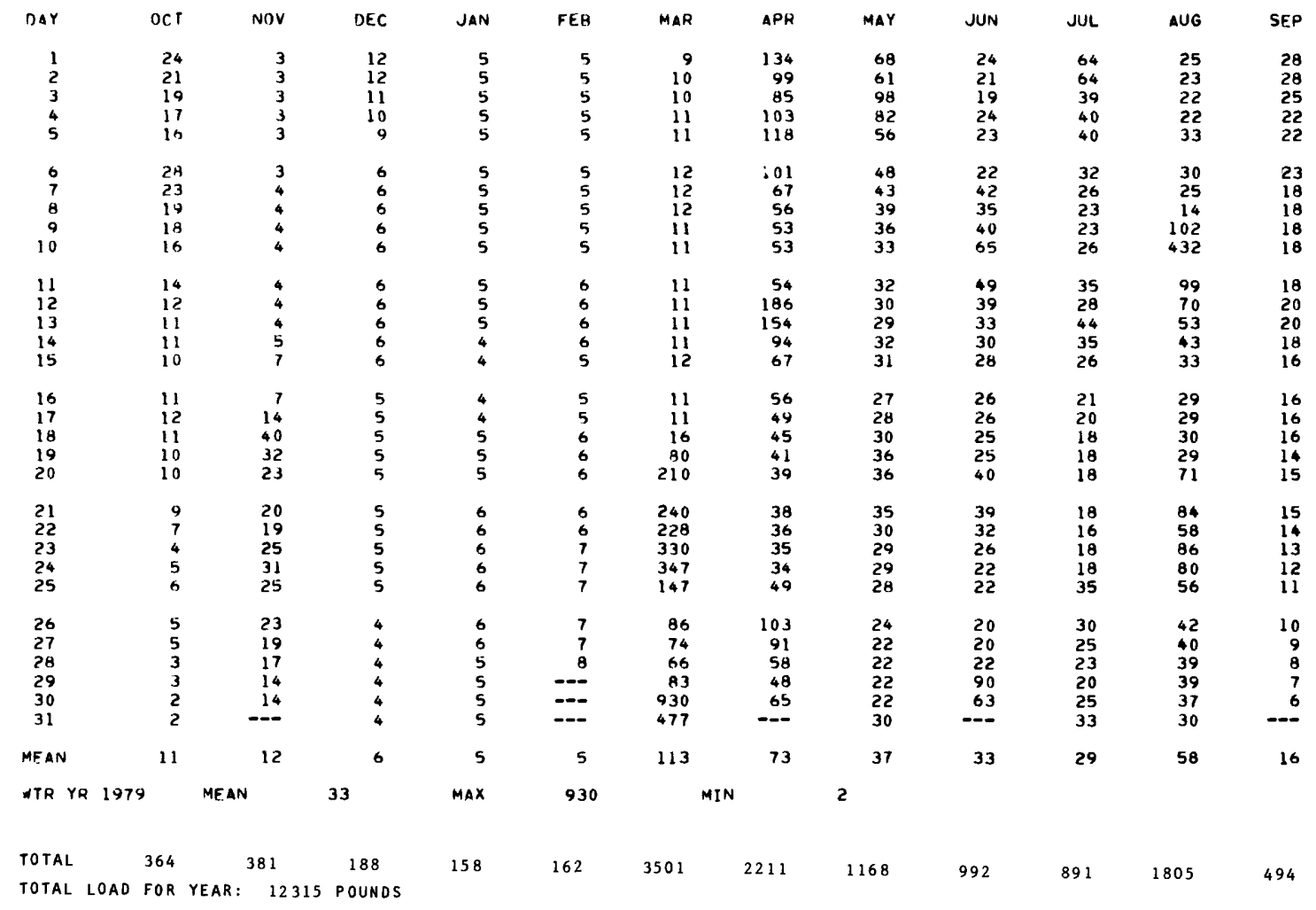

\begin{tabular}{|c|c|c|c|c|c|c|c|c|c|c|c|c|}
\hline & & & TOTAL & OSPHOR & WATER ${ }_{M E}$ & $\begin{array}{l}A R O C T \text { OCT } \\
\text { VALUUE }\end{array}$ & 1978 & TO SEPTEM & R 198 & & & \\
\hline DAY & OCT & Nov & DEC & JAN & FEB & MAH & APH & MaY & JUN & JUL & AUG & SEP \\
\hline $\begin{array}{l}1 \\
2 \\
3 \\
4 \\
5\end{array}$ & $\begin{array}{l}4 \\
6 \\
b \\
6 \\
6\end{array}$ & $\begin{array}{r}10 \\
9 \\
7 \\
5 \\
6\end{array}$ & $\begin{array}{c}10 \\
6 \\
6 \\
6 \\
7\end{array}$ & $\begin{array}{l}9 \\
8 \\
6 \\
6 \\
5\end{array}$ & $\begin{array}{r}9 \\
10 \\
10 \\
11 \\
11\end{array}$ & $\begin{array}{l}6 \\
6 \\
6 \\
6 \\
6\end{array}$ & $\begin{array}{l}28 \\
28 \\
34 \\
57 \\
64\end{array}$ & 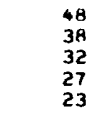 & $\begin{array}{l}48 \\
40 \\
40 \\
36 \\
60\end{array}$ & $\begin{array}{r}10 \\
10 \\
9 \\
9 \\
11\end{array}$ & $\begin{array}{l}11 \\
12 \\
13 \\
12 \\
14\end{array}$ & $\begin{array}{l}43 \\
50 \\
32 \\
28 \\
24\end{array}$ \\
\hline $\begin{array}{r}6 \\
7 \\
8 \\
9 \\
10\end{array}$ & $\begin{array}{r}7 \\
8 \\
10 \\
9 \\
9\end{array}$ & $\begin{array}{r}10 \\
10 \\
10 \\
7 \\
7\end{array}$ & $\begin{array}{r}9 \\
9 \\
8 \\
11 \\
13\end{array}$ & $\begin{array}{l}4 \\
4 \\
4 \\
3 \\
3\end{array}$ & $\begin{array}{l}11 \\
12 \\
12 \\
11 \\
11\end{array}$ & $\begin{array}{l}6 \\
5 \\
5 \\
6 \\
7\end{array}$ & $\begin{array}{r}52 \\
60 \\
380 \\
256 \\
131\end{array}$ & $\begin{array}{l}21 \\
16 \\
16 \\
16 \\
15\end{array}$ & $\begin{array}{r}144 \\
1197 \\
283 \\
175 \\
52\end{array}$ & $\begin{array}{r}10 \\
10 \\
10 \\
10 \\
9\end{array}$ & $\begin{array}{r}13 \\
18 \\
233 \\
152 \\
92\end{array}$ & $\begin{array}{l}22 \\
22 \\
20 \\
26 \\
24\end{array}$ \\
\hline $\begin{array}{l}11 \\
12 \\
13 \\
14 \\
15\end{array}$ & $\begin{array}{r}4 \\
10 \\
7 \\
6 \\
7\end{array}$ & $\begin{array}{c}15 \\
6 \\
6 \\
6 \\
6\end{array}$ & $\begin{array}{r}15 \\
12 \\
8 \\
4 \\
4\end{array}$ & $\begin{array}{l}3 \\
3 \\
3 \\
2 \\
2 \\
2\end{array}$ & $\begin{array}{l}11 \\
12 \\
12 \\
12 \\
12\end{array}$ & $\begin{array}{l}8 \\
7 \\
7 \\
7 \\
8\end{array}$ & $\begin{array}{l}93 \\
73 \\
62 \\
55 \\
60\end{array}$ & $\begin{array}{l}20 \\
15 \\
26 \\
44 \\
48\end{array}$ & $\begin{array}{l}29 \\
25 \\
23 \\
28 \\
32\end{array}$ & $\begin{array}{r}8 \\
8 \\
\theta \\
8 \\
10\end{array}$ & $\begin{array}{l}55 \\
45 \\
35 \\
31 \\
25\end{array}$ & $\begin{array}{l}20 \\
12 \\
25 \\
28 \\
16\end{array}$ \\
\hline $\begin{array}{l}16 \\
17 \\
18 \\
19 \\
20\end{array}$ & 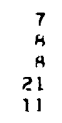 & $\begin{array}{l}6 \\
6 \\
0 \\
6 \\
6\end{array}$ & $\begin{array}{l}3 \\
3 \\
3 \\
4 \\
4\end{array}$ & $\begin{array}{r}16 \\
170 \\
150 \\
58 \\
36\end{array}$ & $\begin{array}{l}11 \\
11 \\
11 \\
11 \\
11\end{array}$ & $\begin{array}{r}16 \\
54 \\
110 \\
95 \\
80\end{array}$ & $\begin{array}{l}84 \\
68 \\
56 \\
51 \\
45\end{array}$ & $\begin{array}{l}44 \\
37 \\
58 \\
67 \\
47\end{array}$ & $\begin{array}{l}23 \\
20 \\
18 \\
33 \\
41\end{array}$ & $\begin{array}{r}16 \\
16 \\
10 \\
10 \\
181\end{array}$ & $\begin{array}{r}20 \\
22 \\
24 \\
22 \\
130\end{array}$ & $\begin{array}{l}23 \\
31 \\
19 \\
13 \\
34\end{array}$ \\
\hline $\begin{array}{l}21 \\
22 \\
23 \\
24 \\
25\end{array}$ & $\begin{array}{l}12 \\
16 \\
29 \\
37 \\
26\end{array}$ & $\begin{array}{l}11 \\
23 \\
22 \\
14 \\
11\end{array}$ & $\begin{array}{r}5 \\
6 \\
12 \\
54 \\
146\end{array}$ & $\begin{array}{l}25 \\
18 \\
16 \\
12 \\
10\end{array}$ & $\begin{array}{l}18 \\
28 \\
20 \\
18 \\
16\end{array}$ & $\begin{array}{l}56 \\
40 \\
27 \\
23 \\
29\end{array}$ & $\begin{array}{l}41 \\
37 \\
30 \\
27 \\
25\end{array}$ & $\begin{array}{l}34 \\
30 \\
25 \\
23 \\
23\end{array}$ & $\begin{array}{l}28 \\
21 \\
19 \\
16 \\
15\end{array}$ & $\begin{array}{r}137 \\
75 \\
36 \\
28 \\
20\end{array}$ & $\begin{array}{r}160 \\
97 \\
45 \\
36 \\
36\end{array}$ & $\begin{array}{r}31 \\
643 \\
231 \\
87 \\
50\end{array}$ \\
\hline $\begin{array}{l}26 \\
27 \\
28 \\
29 \\
30 \\
31\end{array}$ & $\begin{array}{l}16 \\
16 \\
15 \\
12 \\
12 \\
11\end{array}$ & $\begin{array}{r}27 \\
50 \\
36 \\
27 \\
10 \\
---\end{array}$ & $\begin{array}{l}79 \\
29 \\
16 \\
13 \\
12 \\
10\end{array}$ & $\begin{array}{l}10 \\
10 \\
10 \\
10 \\
10 \\
11\end{array}$ & $\begin{array}{r}11 \\
11 \\
10 \\
10 \\
-1 \\
-\because-\end{array}$ & $\begin{array}{l}33 \\
29 \\
27 \\
40 \\
29 \\
29\end{array}$ & $\begin{array}{r}25 \\
23 \\
23 \\
31 \\
45 \\
---\end{array}$ & $\begin{array}{l}20 \\
19 \\
20 \\
50 \\
57 \\
60\end{array}$ & $\begin{array}{r}14 \\
11 \\
14 \\
14 \\
10 \\
---\end{array}$ & $\begin{array}{l}19 \\
15 \\
13 \\
12 \\
11 \\
13\end{array}$ & $\begin{array}{l}60 \\
53 \\
70 \\
81 \\
60 \\
43\end{array}$ & $\begin{array}{r}41 \\
31 \\
24 \\
21 \\
20 \\
--\end{array}$ \\
\hline MEAN & $" 1$ & 12 & 17 & 20 & 12 & 26 & 68 & 32 & 50 & 24 & 55 & 56 \\
\hline WTR YR 1980 & & EAN & 32 & $\max$ & 643 & \multicolumn{2}{|c|}{ MIN } & 2 & & & & \\
\hline AL & 352 & 381 & 527 & 637 & 370 & 813 & 2044 & 1019 & 1499 & 750 & 1720 & 1691 \\
\hline
\end{tabular}


Table 23. Total orthophosphate phosphorus load, in pounds per day, for Onion River at Hingham, 1979 and 1980 water years

TOTAL ORTHOPHOSPHORUS, WATER YEAR OCTOBER 1978 TO SEPTEMEER 1979

\begin{tabular}{|c|c|c|c|c|c|c|c|c|c|c|c|}
\hline OCT & Nov & $D E C$ & JAN & FEB & MaR & APR & mar & JUN & JUL & AUG & SEP \\
\hline $\begin{array}{l}18 \\
17 \\
15 \\
14 \\
13\end{array}$ & $\begin{array}{l}3 \\
3 \\
3 \\
3 \\
3\end{array}$ & $\begin{array}{r}11 \\
10 \\
10 \\
9 \\
8\end{array}$ & $\begin{array}{l}5 \\
5 \\
5 \\
4 \\
4\end{array}$ & $\begin{array}{l}3 \\
3 \\
3 \\
3 \\
3\end{array}$ & $\begin{array}{r}8 \\
8 \\
9 \\
10 \\
10\end{array}$ & $\begin{array}{l}92 \\
38 \\
30 \\
40 \\
49\end{array}$ & $\begin{array}{l}22 \\
19 \\
37 \\
29 \\
16\end{array}$ & $\begin{array}{l}5 \\
4 \\
3 \\
5 \\
4\end{array}$ & $\begin{array}{l}24 \\
22 \\
12 \\
12 \\
12\end{array}$ & $\begin{array}{l}6 \\
6 \\
5 \\
5 \\
9\end{array}$ & $\begin{array}{l}8 \\
8 \\
6 \\
5 \\
5\end{array}$ \\
\hline $\begin{array}{l}21 \\
18 \\
15 \\
14 \\
13\end{array}$ & $\begin{array}{l}3 \\
4 \\
4 \\
4\end{array}$ & $\begin{array}{l}B \\
A \\
B \\
8 \\
7\end{array}$ & $\begin{array}{l}4 \\
4 \\
4\end{array}$ & $\begin{array}{l}3 \\
3 \\
4 \\
4\end{array}$ & $\begin{array}{l}11 \\
11 \\
11 \\
10 \\
10\end{array}$ & $\begin{array}{l}39 \\
22 \\
17 \\
15 \\
15\end{array}$ & $\begin{array}{r}13 \\
11 \\
10 \\
9 \\
8\end{array}$ & $\begin{array}{r}12 \\
10 \\
12 \\
12\end{array}$ & $\begin{array}{l}9 \\
7 \\
6 \\
6 \\
7\end{array}$ & $\begin{array}{r}8 \\
6 \\
24 \\
174\end{array}$ & 6 \\
\hline $\begin{array}{c}12 \\
11 \\
10 \\
10 \\
9\end{array}$ & $\begin{array}{l}4 \\
4 \\
5 \\
6\end{array}$ & $\begin{array}{l}7 \\
6 \\
6 \\
6 \\
6\end{array}$ & 4 & $\begin{array}{l}4 \\
3 \\
3\end{array}$ & $\begin{array}{l}10 \\
10 \\
10 \\
10 \\
11\end{array}$ & $\begin{array}{l}16 \\
94 \\
72 \\
35 \\
22\end{array}$ & $\begin{array}{l}7 \\
7 \\
6 \\
7 \\
7\end{array}$ & $\begin{array}{r}16 \\
12 \\
9 \\
8 \\
8\end{array}$ & $\begin{array}{r}10 \\
8 \\
14 \\
10 \\
7\end{array}$ & $\begin{array}{l}74 \\
38 \\
22 \\
15 \\
10\end{array}$ & 4 \\
\hline $\begin{array}{l}16 \\
17 \\
18 \\
19 \\
20\end{array}$ & $\begin{array}{c}6 \\
12 \\
28 \\
23 \\
18\end{array}$ & $\begin{array}{l}6 \\
6 \\
6 \\
6 \\
6\end{array}$ & $\begin{array}{l}4 \\
5 \\
5 \\
5\end{array}$ & $\begin{array}{l}3 \\
3 \\
4 \\
4 \\
4\end{array}$ & $\begin{array}{r}10 \\
10 \\
9 \\
36 \\
120\end{array}$ & $\begin{array}{l}16 \\
13 \\
12 \\
10 \\
10\end{array}$ & $\begin{array}{l}6 \\
6 \\
? \\
9 \\
9\end{array}$ & $\begin{array}{l}7 \\
7 \\
6 \\
6 \\
6\end{array}$ & $\begin{array}{l}5 \\
5 \\
4 \\
4\end{array}$ & $\begin{array}{r}7 \\
6 \\
5 \\
26\end{array}$ & 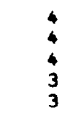 \\
\hline $\begin{array}{l}21 \\
22 \\
23 \\
24 \\
25\end{array}$ & $\begin{array}{l}16 \\
15 \\
19 \\
23 \\
19\end{array}$ & $\begin{array}{l}6 \\
5 \\
5 \\
5 \\
5\end{array}$ & $\begin{array}{l}5 \\
5 \\
4\end{array}$ & $\begin{array}{l}4 \\
5 \\
5 \\
5\end{array}$ & $\begin{array}{r}170 \\
171 \\
204 \\
178 \\
65\end{array}$ & $\begin{array}{r}9 \\
9 \\
8 \\
8 \\
13\end{array}$ & $\begin{array}{l}8 \\
7 \\
6 \\
6 \\
5\end{array}$ & $\begin{array}{r}12 \\
9 \\
7 \\
5 \\
5\end{array}$ & $\begin{array}{l}4 \\
40 \\
10\end{array}$ & $\begin{array}{l}32 \\
20 \\
33 \\
30 \\
19\end{array}$ & \\
\hline $\begin{array}{l}26 \\
27 \\
28 \\
29 \\
30 \\
31\end{array}$ & $\begin{array}{c}18 \\
15 \\
14 \\
12 \\
12 \\
-9\end{array}$ & $\begin{array}{l}5 \\
5 \\
5 \\
5 \\
5 \\
5\end{array}$ & $\begin{array}{l}4 \\
4 \\
3 \\
3\end{array}$ & $\begin{array}{r}5 \\
6 \\
\quad 7 \\
\hdashline- \\
\hdashline- \\
\hdashline-\end{array}$ & $\begin{array}{r}32 \\
22 \\
18 \\
21 \\
210 \\
275 \\
275\end{array}$ & $\begin{array}{r}40 \\
33 \\
18 \\
13 \\
21 \\
\cdots\end{array}$ & $\begin{array}{l}5 \\
4 \\
4 \\
7\end{array}$ & $\begin{array}{r}5 \\
5 \\
5 \\
25 \\
30 \\
-\because-\end{array}$ & $\begin{array}{l}8 \\
6 \\
6 \\
5 \\
6 \\
9\end{array}$ & $\begin{array}{l}13 \\
12 \\
12 \\
12 \\
11 \\
8\end{array}$ & $\begin{array}{r}3 \\
-4 \\
-\cdots\end{array}$ \\
\hline $\begin{array}{r}21 \\
3.0\end{array}$ & $\begin{array}{r}28 \\
3.0\end{array}$ & $\begin{array}{r}11 \\
5.0\end{array}$ & $\begin{array}{l}5.0 \\
3.0\end{array}$ & $\begin{array}{l}7: 0 \\
3: 0\end{array}$ & $\begin{array}{l}275 \\
8.0\end{array}$ & $\begin{array}{r}94 \\
8.0\end{array}$ & $\begin{array}{r}37 \\
4.0\end{array}$ & $\begin{array}{r}30 \\
3.0\end{array}$ & $\begin{array}{r}24 \\
4.00\end{array}$ & $\begin{array}{r}174 \\
4.00\end{array}$ & $\begin{array}{l}8.0 \\
3.0\end{array}$ \\
\hline
\end{tabular}

WTR YR 1979 MAX 275 MIN 3.0

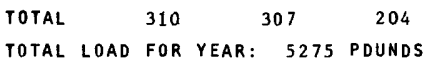
TOTAL ORTHOPHOSPHORUS, WATER YEAR OCTOBER 1979 TO SEPTEMBER 1980
MEAN VALUES

\begin{tabular}{|c|c|c|c|c|c|c|c|c|c|c|c|c|}
\hline DAY & OCT & NOV & DEC & JAN & FEB & MAR & $\triangle P R$ & Mar & JUN & JUL & AUG & SEP \\
\hline $\begin{array}{l}1 \\
2 \\
3 \\
4 \\
5\end{array}$ & $\begin{array}{l}3 \\
4 \\
3 \\
4 \\
4\end{array}$ & $\begin{array}{r}10 \\
9 \\
7 \\
6 \\
6\end{array}$ & $\begin{array}{l}9 \\
7 \\
6 \\
7 \\
7\end{array}$ & $\begin{array}{l}9 \\
8 \\
7 \\
6 \\
6\end{array}$ & $\begin{array}{l}6 \\
6 \\
6 \\
7 \\
8\end{array}$ & $\begin{array}{l}6 \\
6 \\
6 \\
6 \\
6\end{array}$ & $\begin{array}{l}10 \\
10 \\
11 \\
19 \\
22\end{array}$ & $\begin{array}{l}8 \\
6 \\
4 \\
4\end{array}$ & $\begin{array}{l}8 \\
6 \\
6 \\
5 \\
6\end{array}$ & $\begin{array}{l}3 \\
3 \\
2 \\
2 \\
3\end{array}$ & $\begin{array}{l}3 \\
3 \\
4 \\
3 \\
4\end{array}$ & $\begin{array}{r}16 \\
20 \\
11 \\
9 \\
8\end{array}$ \\
\hline $\begin{array}{r}6 \\
7 \\
8 \\
9 \\
10\end{array}$ & $\begin{array}{l}4 \\
5 \\
6 \\
6 \\
5\end{array}$ & $\begin{array}{r}10 \\
9 \\
9 \\
7 \\
7\end{array}$ & $\begin{array}{r}9 \\
9 \\
8 \\
11 \\
12\end{array}$ & $\begin{array}{l}5 \\
5 \\
5 \\
4 \\
4\end{array}$ & $\begin{array}{l}8 \\
8 \\
A \\
8 \\
8\end{array}$ & $\begin{array}{l}4 \\
3 \\
3 \\
4 \\
4\end{array}$ & $\begin{array}{r}18 \\
22 \\
101 \\
85 \\
48\end{array}$ & $\begin{array}{l}2 \\
2 \\
2 \\
2 \\
2\end{array}$ & $\begin{array}{l}39 \\
43 \\
97 \\
69 \\
19\end{array}$ & $\begin{array}{l}3 \\
3 \\
3 \\
3 \\
2\end{array}$ & $\begin{array}{r}13 \\
13 \\
226 \\
138 \\
63\end{array}$ & $\begin{array}{l}7 \\
7 \\
6 \\
8 \\
8\end{array}$ \\
\hline $\begin{array}{l}11 \\
12 \\
13 \\
14 \\
15\end{array}$ & $\begin{array}{l}5 \\
6 \\
4 \\
4 \\
4\end{array}$ & $\begin{array}{r}13 \\
6 \\
7 \\
7 \\
7\end{array}$ & $\begin{array}{r}13 \\
11 \\
8 \\
4 \\
5\end{array}$ & $\begin{array}{l}4 \\
4 \\
3 \\
3\end{array}$ & $\begin{array}{l}8 \\
8 \\
8 \\
8 \\
8\end{array}$ & $\begin{array}{l}4 \\
4 \\
4 \\
4\end{array}$ & $\begin{array}{l}31 \\
24 \\
21 \\
18 \\
20\end{array}$ & $\begin{array}{l}2 \\
2 \\
3 \\
7 \\
8\end{array}$ & $\begin{array}{r}10 \\
8 \\
8 \\
10 \\
11\end{array}$ & $\begin{array}{l}2 \\
2 \\
2 \\
2 \\
3\end{array}$ & $\begin{array}{l}30 \\
24 \\
19 \\
16 \\
13\end{array}$ & $\begin{array}{r}6 \\
10 \\
21 \\
24 \\
14\end{array}$ \\
\hline $\begin{array}{l}16 \\
17 \\
18 \\
19 \\
20\end{array}$ & $\begin{array}{r}4 \\
5 \\
5 \\
12 \\
7\end{array}$ & $\begin{array}{l}6 \\
7 \\
7 \\
7 \\
7\end{array}$ & $\begin{array}{l}4 \\
4 \\
5 \\
5\end{array}$ & $\begin{array}{l}11 \\
51 \\
46 \\
20 \\
15\end{array}$ & $\begin{array}{l}8 \\
8 \\
7 \\
8 \\
9\end{array}$ & $\begin{array}{r}5 \\
38 \\
79 \\
41 \\
30\end{array}$ & $\begin{array}{l}28 \\
23 \\
19 \\
17 \\
15\end{array}$ & $\begin{array}{r}7 \\
5 \\
10 \\
12 \\
7\end{array}$ & $\begin{array}{r}8 \\
6 \\
6 \\
12 \\
16\end{array}$ & $\begin{array}{r}5 \\
4 \\
3 \\
66\end{array}$ & $\begin{array}{r}10 \\
10 \\
10 \\
8 \\
60\end{array}$ & $\begin{array}{l}19 \\
26 \\
16 \\
11 \\
29\end{array}$ \\
\hline $\begin{array}{l}21 \\
22 \\
23 \\
24 \\
25\end{array}$ & $\begin{array}{r}7 \\
9 \\
16 \\
20 \\
15\end{array}$ & $\begin{array}{l}11 \\
19 \\
18 \\
13 \\
11\end{array}$ & $\begin{array}{r}6 \\
7 \\
11 \\
36 \\
80\end{array}$ & $\begin{array}{r}11 \\
11 \\
22 \\
17 \\
9\end{array}$ & $\begin{array}{r}9 \\
19 \\
14 \\
14 \\
12\end{array}$ & $\begin{array}{r}21 \\
15 \\
11 \\
9 \\
12\end{array}$ & $\begin{array}{r}14 \\
12 \\
10 \\
9 \\
8\end{array}$ & $\begin{array}{l}5 \\
4 \\
3 \\
3 \\
3\end{array}$ & $\begin{array}{r}10 \\
7 \\
6 \\
5 \\
5\end{array}$ & $\begin{array}{r}55 \\
27 \\
8 \\
6 \\
5\end{array}$ & $\begin{array}{l}78 \\
43 \\
17 \\
13 \\
13\end{array}$ & $\begin{array}{r}26 \\
243 \\
107 \\
51 \\
31\end{array}$ \\
\hline $\begin{array}{l}26 \\
27 \\
28 \\
29 \\
30 \\
31\end{array}$ & $\begin{array}{l}4 \\
9 \\
9 \\
7 \\
7 \\
7\end{array}$ & $\begin{array}{r}21 \\
34 \\
26 \\
21 \\
10 \\
-\end{array}$ & $\begin{array}{c}39 \\
22 \\
14 \\
12 \\
11 \\
9\end{array}$ & $\begin{array}{l}8 \\
8 \\
7 \\
7 \\
7 \\
5\end{array}$ & \begin{tabular}{l}
11 \\
11 \\
11 \\
11 \\
\hdashline-0
\end{tabular} & $\begin{array}{l}14 \\
12 \\
11 \\
10 \\
10 \\
10\end{array}$ & $\begin{array}{r}8 \\
8 \\
8 \\
6 \\
10 \\
-0\end{array}$ & $\begin{array}{r}2 \\
2 \\
2 \\
8 \\
10 \\
10\end{array}$ & $\begin{array}{r}4 \\
3 \\
4 \\
3 \\
-0\end{array}$ & $\begin{array}{l}5 \\
5 \\
4 \\
3 \\
3 \\
4\end{array}$ & $\begin{array}{l}24 \\
21 \\
29 \\
34 \\
24 \\
16\end{array}$ & $\begin{array}{r}22 \\
18 \\
16 \\
13 \\
13 \\
-\end{array}$ \\
\hline $\begin{array}{l}\operatorname{MAx} \\
\operatorname{MIN}\end{array}$ & $\begin{array}{r}20 \\
3.0\end{array}$ & $\begin{array}{r}34 \\
6.0\end{array}$ & $\begin{array}{r}80 \\
4.0\end{array}$ & $\begin{array}{r}51 \\
3.0\end{array}$ & $\begin{array}{r}19 \\
6.0\end{array}$ & $\begin{array}{r}79 \\
3.0\end{array}$ & $\begin{array}{l}101 \\
6.0\end{array}$ & $\begin{array}{r}12 \\
2.0\end{array}$ & $\begin{array}{r}97 \\
3.0\end{array}$ & $\begin{array}{r}66 \\
2.0\end{array}$ & $\begin{array}{l}226 \\
3.0\end{array}$ & $\begin{array}{l}243 \\
6.0\end{array}$ \\
\hline
\end{tabular}

WTR YR 1980 MAX 243 MIN 2.0

IOTAL $215 \quad 338 \quad 395$

TOTAL LOAD FOR YEAR: 5223 POUNDS 
Table 24. Total organic nitrogen load, in pounds per day, for Onion River at Hingham, 1979 and 1980 water years TOTAL ORGANIC NITROGEN, WATER YEAR OCTOBER 1978 TO SEPTEMBER 1979

\begin{tabular}{|c|c|c|c|c|c|c|c|c|c|c|c|c|}
\hline DAY & OCT & NOV & DEC & JAN & FEB & MAR & APH & MAY & JUN & JUL & AUG & SEP \\
\hline $\begin{array}{l}1 \\
2 \\
3 \\
4 \\
5\end{array}$ & $\begin{array}{r}1080 \\
830 \\
540 \\
400 \\
300\end{array}$ & $\begin{array}{l}96 \\
94 \\
94 \\
94 \\
94\end{array}$ & $\begin{array}{l}300 \\
290 \\
280 \\
200 \\
260\end{array}$ & $\begin{array}{l}48 \\
48 \\
48 \\
45 \\
45\end{array}$ & $\begin{array}{l}28 \\
28 \\
28 \\
28 \\
28\end{array}$ & $\begin{array}{l}53 \\
55 \\
80 \\
97 \\
97\end{array}$ & $\begin{array}{l}6250 \\
3330 \\
2320 \\
2270 \\
2510\end{array}$ & $\begin{array}{l}2230 \\
1850 \\
2620 \\
1730 \\
1370\end{array}$ & $\begin{array}{l}250 \\
260 \\
210 \\
630 \\
500\end{array}$ & $\begin{array}{l}985 \\
794 \\
730 \\
500 \\
470\end{array}$ & $\begin{array}{r}160 \\
120 \\
110 \\
92 \\
92\end{array}$ & $\begin{array}{l}380 \\
340 \\
290 \\
220 \\
190\end{array}$ \\
\hline $\begin{array}{r}6 \\
7 \\
8 \\
9 \\
10\end{array}$ & $\begin{array}{r}1280 \\
1200 \\
940 \\
760 \\
600\end{array}$ & $\begin{array}{l}94 \\
94 \\
94 \\
94 \\
94\end{array}$ & $\begin{array}{l}240 \\
230 \\
220 \\
220 \\
220\end{array}$ & $\begin{array}{l}45 \\
43 \\
43 \\
43 \\
43\end{array}$ & $\begin{array}{l}28 \\
29 \\
29 \\
31 \\
32\end{array}$ & $\begin{array}{l}95 \\
95 \\
95 \\
95 \\
95\end{array}$ & $\begin{array}{r}1980 \\
1580 \\
1120 \\
960 \\
940\end{array}$ & $\begin{array}{r}1140 \\
910 \\
740 \\
640 \\
550\end{array}$ & $\begin{array}{r}450 \\
1030 \\
840 \\
680 \\
1690\end{array}$ & $\begin{array}{l}420 \\
330 \\
290 \\
260 \\
230\end{array}$ & $\begin{array}{r}170 \\
150 \\
97 \\
130 \\
3340\end{array}$ & $\begin{array}{r}180 \\
160 \\
120 \\
100 \\
92\end{array}$ \\
\hline $\begin{array}{l}11 \\
12 \\
13 \\
14 \\
15\end{array}$ & $\begin{array}{l}480 \\
420 \\
330 \\
270 \\
220\end{array}$ & $\begin{array}{r}94 \\
94 \\
120 \\
160 \\
240\end{array}$ & $\begin{array}{l}220 \\
210 \\
200 \\
190 \\
180\end{array}$ & $\begin{array}{l}43 \\
43 \\
43 \\
32 \\
28\end{array}$ & $\begin{array}{l}32 \\
32 \\
32 \\
32 \\
32\end{array}$ & $\begin{array}{r}95 \\
99 \\
110 \\
110 \\
110\end{array}$ & $\begin{array}{l}1000 \\
3310 \\
3980 \\
2990 \\
1810\end{array}$ & $\begin{array}{l}490 \\
470 \\
440 \\
420 \\
460\end{array}$ & $\begin{array}{r}1190 \\
870 \\
710 \\
590 \\
530\end{array}$ & $\begin{array}{l}280 \\
330 \\
290 \\
390 \\
330\end{array}$ & $\begin{array}{r}4590 \\
2120 \\
778 \\
497 \\
348\end{array}$ & $\begin{array}{r}92 \\
100 \\
100 \\
92 \\
85\end{array}$ \\
\hline $\begin{array}{l}16 \\
17 \\
18 \\
19 \\
20\end{array}$ & $\begin{array}{l}240 \\
240 \\
230 \\
210 \\
220\end{array}$ & $\begin{array}{r}190 \\
430 \\
1280 \\
1080 \\
730\end{array}$ & $\begin{array}{l}180 \\
170 \\
160 \\
160 \\
160\end{array}$ & $\begin{array}{l}26 \\
28 \\
32 \\
37 \\
39\end{array}$ & $\begin{array}{l}31 \\
31 \\
32 \\
32 \\
32\end{array}$ & $\begin{array}{r}110 \\
110 \\
170 \\
810 \\
2470\end{array}$ & $\begin{array}{r}1180 \\
940 \\
780 \\
680 \\
600\end{array}$ & $\begin{array}{l}380 \\
420 \\
470 \\
800 \\
760\end{array}$ & $\begin{array}{l}500 \\
450 \\
420 \\
410 \\
870\end{array}$ & $\begin{array}{l}250 \\
200 \\
180 \\
190 \\
190\end{array}$ & $\begin{array}{l}246 \\
202 \\
182 \\
168 \\
448\end{array}$ & $\begin{array}{l}78 \\
78 \\
71 \\
64 \\
58\end{array}$ \\
\hline $\begin{array}{l}21 \\
22 \\
23 \\
24 \\
25\end{array}$ & $\begin{array}{l}210 \\
160 \\
100 \\
210 \\
260\end{array}$ & $\begin{array}{r}510 \\
380 \\
570 \\
1280 \\
970\end{array}$ & $\begin{array}{l}150 \\
150 \\
150 \\
140 \\
140\end{array}$ & $\begin{array}{l}41 \\
41 \\
41 \\
41 \\
41\end{array}$ & $\begin{array}{l}34 \\
36 \\
39 \\
42 \\
45\end{array}$ & $\begin{array}{r}8660 \\
9110 \\
10700 \\
9780 \\
3560\end{array}$ & $\begin{array}{l}550 \\
520 \\
480 \\
440 \\
540\end{array}$ & $\begin{array}{l}600 \\
490 \\
420 \\
380 \\
356\end{array}$ & $\begin{array}{l}710 \\
470 \\
360 \\
290 \\
260\end{array}$ & $\begin{array}{l}200 \\
220 \\
190 \\
180 \\
200\end{array}$ & $\begin{array}{r}1930 \\
1300 \\
950 \\
2680 \\
2100\end{array}$ & $\begin{array}{l}64 \\
64 \\
64 \\
64 \\
64\end{array}$ \\
\hline $\begin{array}{l}26 \\
27 \\
28 \\
29 \\
30 \\
31\end{array}$ & $\begin{array}{r}240 \\
160 \\
120 \\
79 \\
79 \\
86\end{array}$ & $\begin{array}{l}730 \\
540 \\
460 \\
390 \\
350 \\
-\end{array}$ & $\begin{array}{l}140 \\
140 \\
140 \\
130 \\
130 \\
130\end{array}$ & $\begin{array}{l}39 \\
39 \\
39 \\
39 \\
39 \\
39\end{array}$ & $\begin{array}{r}47 \\
49 \\
52 \\
--- \\
--- \\
---\end{array}$ & $\begin{array}{r}2110 \\
1610 \\
1430 \\
1430 \\
5280 \\
10800\end{array}$ & $\begin{array}{r}1410 \\
1690 \\
1140 \\
810 \\
2330 \\
0\end{array}$ & $\begin{array}{l}300 \\
280 \\
280 \\
270 \\
380 \\
420\end{array}$ & $\begin{array}{r}230 \\
220 \\
211 \\
817 \\
1680 \\
-\end{array}$ & $\begin{array}{r}160 \\
140 \\
110 \\
92 \\
85 \\
130\end{array}$ & $\begin{array}{l}920 \\
660 \\
620 \\
520 \\
450 \\
370\end{array}$ & $\begin{array}{l}64 \\
11 \\
64 \\
58 \\
64 \\
-\end{array}$ \\
\hline $\begin{array}{l}\text { MAX } \\
\text { MIN }\end{array}$ & $\begin{array}{r}1280 \\
79\end{array}$ & $\begin{array}{r}1280 \\
86\end{array}$ & $\begin{array}{l}300 \\
130\end{array}$ & $\begin{array}{l}48 \\
26\end{array}$ & $\begin{array}{l}52 \\
28\end{array}$ & $\begin{array}{r}10800 \\
53\end{array}$ & $\begin{array}{r}6250 \\
440\end{array}$ & $\begin{array}{r}2620 \\
270\end{array}$ & $\begin{array}{r}1690 \\
210\end{array}$ & $\begin{array}{r}985 \\
85\end{array}$ & $\begin{array}{r}4590 \\
92\end{array}$ & $\begin{array}{r}380 \\
58\end{array}$ \\
\hline
\end{tabular}

WTR YR 1979 MAX 10800 MIN 26

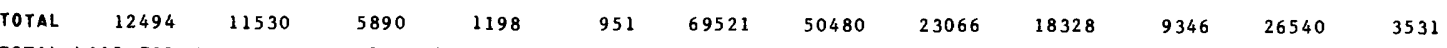
TOTAL LOAD FOR YEAR: 232835 POUNDS

TOTAL ORGANIC NITROGEN. WATER YEAR OCTOBER 1979 TO SEPTEMBER 1980

\begin{tabular}{|c|c|c|c|c|c|c|c|c|c|c|c|c|}
\hline DAY & $\mathrm{OCT}$ & NOV & DEC & JAN & FEB & MAR & APR & MAY & JUN & JUL & AUG & SEP \\
\hline $\begin{array}{l}1 \\
2 \\
3 \\
4 \\
5\end{array}$ & $\begin{array}{l}66 \\
66 \\
73 \\
66 \\
73\end{array}$ & $\begin{array}{r}118 \\
140 \\
120 \\
110 \\
94\end{array}$ & $\begin{array}{l}140 \\
120 \\
110 \\
110 \\
110\end{array}$ & $\begin{array}{l}200 \\
170 \\
160 \\
140 \\
140\end{array}$ & $\begin{array}{l}86 \\
92 \\
94 \\
97 \\
97\end{array}$ & $\begin{array}{l}67 \\
58 \\
52 \\
48 \\
54\end{array}$ & $\begin{array}{r}370 \\
360 \\
400 \\
1500 \\
2940\end{array}$ & $\begin{array}{l}630 \\
550 \\
460 \\
380 \\
330\end{array}$ & $\begin{array}{l}370 \\
320 \\
290 \\
280 \\
379\end{array}$ & $\begin{array}{l}120 \\
120 \\
120 \\
120 \\
110\end{array}$ & $\begin{array}{l}140 \\
130 \\
140 \\
150 \\
150\end{array}$ & $\begin{array}{l}500 \\
470 \\
440 \\
320 \\
290\end{array}$ \\
\hline $\begin{array}{r}6 \\
7 \\
8 \\
9 \\
10\end{array}$ & $\begin{array}{r}80 \\
80 \\
94 \\
110 \\
100\end{array}$ & $\begin{array}{r}100 \\
140 \\
140 \\
130 \\
92\end{array}$ & $\begin{array}{l}110 \\
120 \\
140 \\
240 \\
140\end{array}$ & $\begin{array}{r}130 \\
120 \\
110 \\
100 \\
94\end{array}$ & $\begin{array}{l}94 \\
92 \\
86 \\
76 \\
70\end{array}$ & $\begin{array}{l}56 \\
39 \\
39 \\
41 \\
41\end{array}$ & $\begin{array}{l}1520 \\
1170 \\
2840 \\
5400 \\
4650\end{array}$ & $\begin{array}{l}300 \\
270 \\
248 \\
250 \\
220\end{array}$ & $\begin{array}{l}1450 \\
2590 \\
3160 \\
3140 \\
2580\end{array}$ & $\begin{array}{l}130 \\
130 \\
120 \\
100 \\
100\end{array}$ & $\begin{array}{r}170 \\
172 \\
1930 \\
4280 \\
2260\end{array}$ & $\begin{array}{l}250 \\
230 \\
220 \\
230 \\
260\end{array}$ \\
\hline $\begin{array}{l}11 \\
12 \\
13 \\
14 \\
15\end{array}$ & $\begin{array}{r}94 \\
87 \\
100 \\
80 \\
73\end{array}$ & $\begin{array}{l}86 \\
92 \\
86 \\
86 \\
86\end{array}$ & $\begin{array}{r}120 \\
54 \\
49 \\
47 \\
43\end{array}$ & $\begin{array}{r}87 \\
87 \\
80 \\
80 \\
190\end{array}$ & $\begin{array}{l}68 \\
68 \\
68 \\
65 \\
62\end{array}$ & $\begin{array}{l}39 \\
34 \\
37 \\
39 \\
43\end{array}$ & $\begin{array}{r}3030 \\
1710 \\
1260 \\
980 \\
1000\end{array}$ & $\begin{array}{l}240 \\
240 \\
250 \\
390 \\
550\end{array}$ & $\begin{array}{l}803 \\
397 \\
400 \\
380 \\
440\end{array}$ & $\begin{array}{r}100 \\
92 \\
92 \\
83 \\
100\end{array}$ & $\begin{array}{r}1270 \\
943 \\
721 \\
499 \\
370\end{array}$ & $\begin{array}{l}240 \\
230 \\
350 \\
440 \\
410\end{array}$ \\
\hline $\begin{array}{l}16 \\
17 \\
18 \\
19 \\
20\end{array}$ & $\begin{array}{r}87 \\
87 \\
87 \\
94 \\
190\end{array}$ & $\begin{array}{l}92 \\
96 \\
92 \\
92 \\
92\end{array}$ & $\begin{array}{l}41 \\
41 \\
41 \\
41 \\
43\end{array}$ & $\begin{array}{r}640 \\
3800 \\
2900 \\
2000 \\
1000\end{array}$ & $\begin{array}{l}59 \\
54 \\
51 \\
54 \\
62\end{array}$ & $\begin{array}{r}54 \\
390 \\
510 \\
2300 \\
2350\end{array}$ & $\begin{array}{r}1990 \\
1560 \\
1160 \\
920 \\
770\end{array}$ & $\begin{array}{l}480 \\
420 \\
470 \\
640 \\
600\end{array}$ & $\begin{array}{l}430 \\
300 \\
260 \\
280 \\
480\end{array}$ & $\begin{array}{r}201 \\
355 \\
222 \\
159 \\
2240\end{array}$ & $\begin{array}{l}380 \\
330 \\
340 \\
340 \\
990\end{array}$ & $\begin{array}{l}334 \\
841 \\
817 \\
547 \\
838\end{array}$ \\
\hline $\begin{array}{l}21 \\
22 \\
23 \\
24 \\
25\end{array}$ & $\begin{array}{l}110 \\
100 \\
140 \\
220 \\
260\end{array}$ & $\begin{array}{r}95 \\
210 \\
290 \\
230 \\
170\end{array}$ & $\begin{array}{r}43 \\
47 \\
55 \\
70 \\
1800\end{array}$ & $\begin{array}{l}650 \\
420 \\
270 \\
200 \\
160\end{array}$ & $\begin{array}{r}97 \\
150 \\
260 \\
210 \\
170\end{array}$ & $\begin{array}{r}1030 \\
580 \\
350 \\
310 \\
330\end{array}$ & $\begin{array}{l}650 \\
570 \\
490 \\
410 \\
370\end{array}$ & $\begin{array}{l}440 \\
350 \\
300 \\
280 \\
260\end{array}$ & $\begin{array}{l}440 \\
300 \\
260 \\
200 \\
180\end{array}$ & $\begin{array}{r}3470 \\
1650 \\
813 \\
350 \\
260\end{array}$ & $\begin{array}{r}1750 \\
1200 \\
690 \\
450 \\
380\end{array}$ & $\begin{array}{l}1820 \\
8430 \\
6920 \\
6360 \\
3730\end{array}$ \\
\hline $\begin{array}{l}26 \\
27 \\
28 \\
29 \\
30 \\
31\end{array}$ & $\begin{array}{l}190 \\
140 \\
140 \\
140 \\
110 \\
110\end{array}$ & $\begin{array}{l}230 \\
550 \\
520 \\
290 \\
150 \\
---\end{array}$ & $\begin{array}{r}1600 \\
550 \\
350 \\
290 \\
240 \\
220\end{array}$ & $\begin{array}{l}140 \\
120 \\
120 \\
110 \\
100 \\
100\end{array}$ & $\begin{array}{r}170 \\
160 \\
100 \\
71 \\
\hdashline-0 \\
\hdashline-\end{array}$ & $\begin{array}{l}430 \\
256 \\
300 \\
580 \\
620 \\
460\end{array}$ & $\begin{array}{l}350 \\
220 \\
310 \\
470 \\
580 \\
--0\end{array}$ & $\begin{array}{l}240 \\
210 \\
200 \\
220 \\
380 \\
400\end{array}$ & $\begin{array}{l}170 \\
150 \\
150 \\
170 \\
160 \\
-\cdots\end{array}$ & $\begin{array}{l}230 \\
240 \\
200 \\
170 \\
140 \\
130\end{array}$ & $\begin{array}{l}470 \\
520 \\
484 \\
820 \\
700 \\
540\end{array}$ & $\begin{array}{r}1780 \\
1280 \\
1130 \\
938 \\
728 \\
-\end{array}$ \\
\hline $\begin{array}{l}\operatorname{Max} \\
\operatorname{MIN}\end{array}$ & $\begin{array}{r}260 \\
66\end{array}$ & $\begin{array}{r}550 \\
86\end{array}$ & $\begin{array}{r}1800 \\
41\end{array}$ & $\begin{array}{r}3800 \\
80\end{array}$ & $\begin{array}{r}260 \\
51\end{array}$ & $\begin{array}{r}2350 \\
34\end{array}$ & $\begin{array}{r}5400 \\
220\end{array}$ & $\begin{array}{l}640 \\
200\end{array}$ & $\begin{array}{r}3160 \\
150\end{array}$ & $\begin{array}{r}3470 \\
83\end{array}$ & $\begin{array}{r}4280 \\
130\end{array}$ & $\begin{array}{r}8430 \\
220\end{array}$ \\
\hline
\end{tabular}

WTR YR 1980 MAX $8430 \quad$ MIN 34

TOTAL $3447 \quad 4819 \quad 7125 \quad 14618$

2883

$39950 \quad 11198 \quad 20909$

12467

23709

41373 
Table 25. Total nitrogen load, in pounds per day, for Onion River at Hingham, 1979 and 1980 water years

\begin{tabular}{|c|c|c|c|c|c|c|c|c|c|c|c|c|}
\hline \multirow[b]{2}{*}{ DAY } & \multirow[b]{2}{*}{ DCT } & \multirow[b]{2}{*}{ Nov } & \multirow{2}{*}{$\begin{array}{l}\text { TOTAL } \\
\text { DEC }\end{array}$} & \multirow{2}{*}{$\begin{array}{l}\text { NITROGFN, } \\
\text { JAN }\end{array}$} & \multicolumn{3}{|c|}{ WATER YEAR OCTOBER 1978} & \multicolumn{2}{|c|}{ TO SEPTEMBER 1979} & \multirow[b]{2}{*}{ JUL } & \multirow[b]{2}{*}{ AUG } & \multirow[b]{2}{*}{ SEP } \\
\hline & & & & & FEB & MAR & $A P R$ & MAY & JUN & & & \\
\hline $\begin{array}{l}1 \\
2 \\
3 \\
4 \\
5\end{array}$ & $\begin{array}{r}2400 \\
1850 \\
1210 \\
900 \\
680\end{array}$ & $\begin{array}{l}200 \\
210 \\
210 \\
210 \\
210\end{array}$ & $\begin{array}{l}680 \\
650 \\
630 \\
580 \\
580\end{array}$ & $\begin{array}{l}240 \\
240 \\
240 \\
230 \\
230\end{array}$ & $\begin{array}{l}180 \\
180 \\
180 \\
180 \\
180\end{array}$ & $\begin{array}{l}360 \\
370 \\
400 \\
490 \\
490\end{array}$ & $\begin{array}{r}21300 \\
12600 \\
8220 \\
8030 \\
8960\end{array}$ & $\begin{array}{l}7940 \\
6250 \\
9780 \\
5720 \\
4230\end{array}$ & $\begin{array}{l}470 \\
490 \\
380 \\
720 \\
510\end{array}$ & $\begin{array}{r}2870 \\
1920 \\
1560 \\
860 \\
780\end{array}$ & $\begin{array}{l}350 \\
270 \\
240 \\
200 \\
200\end{array}$ & $\begin{array}{l}850 \\
770 \\
650 \\
490 \\
430\end{array}$ \\
\hline $\begin{array}{r}6 \\
7 \\
8 \\
9 \\
10\end{array}$ & $\begin{array}{l}2830 \\
2660 \\
2080 \\
1700 \\
1340\end{array}$ & $\begin{array}{l}210 \\
210 \\
210 \\
210 \\
210\end{array}$ & $\begin{array}{l}550 \\
530 \\
500 \\
500 \\
500\end{array}$ & $\begin{array}{l}230 \\
220 \\
220 \\
220 \\
220\end{array}$ & $\begin{array}{l}180 \\
190 \\
190 \\
200 \\
220\end{array}$ & $\begin{array}{l}480 \\
480 \\
480 \\
480 \\
480\end{array}$ & $\begin{array}{l}6930 \\
5410 \\
3720 \\
3120 \\
3050\end{array}$ & $\begin{array}{l}3330 \\
2500 \\
1910 \\
1570 \\
1310\end{array}$ & $\begin{array}{l}450 \\
2670 \\
1940 \\
1410 \\
5740\end{array}$ & $\begin{array}{l}670 \\
460 \\
370 \\
320 \\
270\end{array}$ & $\begin{array}{l}390 \\
330 \\
154 \\
217 \\
6950\end{array}$ & $\begin{array}{l}410 \\
350 \\
250 \\
220 \\
200\end{array}$ \\
\hline $\begin{array}{l}11 \\
12 \\
13 \\
14 \\
15\end{array}$ & $\begin{array}{r}1080 \\
930 \\
730 \\
600 \\
500\end{array}$ & $\begin{array}{l}210 \\
210 \\
270 \\
370 \\
550\end{array}$ & $\begin{array}{l}500 \\
480 \\
460 \\
430 \\
410\end{array}$ & $\begin{array}{l}220 \\
220 \\
220 \\
160 \\
140\end{array}$ & $\begin{array}{l}220 \\
220 \\
220 \\
220 \\
220\end{array}$ & $\begin{array}{l}480 \\
500 \\
530 \\
540 \\
540\end{array}$ & $\begin{array}{r}3280 \\
12120 \\
14840 \\
10850 \\
6260\end{array}$ & $\begin{array}{r}1130 \\
1070 \\
960 \\
900 \\
1020\end{array}$ & $\begin{array}{l}3350 \\
2050 \\
1510 \\
1120 \\
950\end{array}$ & $\begin{array}{l}350 \\
460 \\
370 \\
600 \\
460\end{array}$ & $\begin{array}{r}10600 \\
4910 \\
1840 \\
1190 \\
843\end{array}$ & $\begin{array}{l}200 \\
220 \\
220 \\
200 \\
190\end{array}$ \\
\hline $\begin{array}{l}16 \\
17 \\
18 \\
19 \\
20\end{array}$ & $\begin{array}{l}550 \\
550 \\
530 \\
480 \\
500\end{array}$ & $\begin{array}{r}430 \\
960 \\
2830 \\
2400 \\
1620\end{array}$ & $\begin{array}{l}410 \\
390 \\
370 \\
370 \\
370\end{array}$ & $\begin{array}{l}130 \\
140 \\
160 \\
180 \\
190\end{array}$ & $\begin{array}{l}200 \\
200 \\
220 \\
220 \\
220\end{array}$ & $\begin{array}{r}540 \\
540 \\
1800 \\
4510 \\
9380\end{array}$ & $\begin{array}{l}3930 \\
3050 \\
2500 \\
2150 \\
1880\end{array}$ & $\begin{array}{r}800 \\
900 \\
1070 \\
2130 \\
1980\end{array}$ & $\begin{array}{r}860 \\
740 \\
670 \\
630 \\
2050\end{array}$ & $\begin{array}{l}290 \\
220 \\
170 \\
200 \\
200\end{array}$ & $\begin{array}{l}603 \\
500 \\
456 \\
428 \\
898\end{array}$ & $\begin{array}{l}170 \\
170 \\
160 \\
140 \\
130\end{array}$ \\
\hline $\begin{array}{l}21 \\
22 \\
23 \\
24 \\
25\end{array}$ & $\begin{array}{l}480 \\
370 \\
230 \\
480 \\
580\end{array}$ & $\begin{array}{r}1150 \\
840 \\
1280 \\
2830 \\
2160\end{array}$ & $\begin{array}{l}350 \\
350 \\
350 \\
330 \\
330\end{array}$ & $\begin{array}{l}200 \\
200 \\
200 \\
200 \\
200\end{array}$ & $\begin{array}{l}230 \\
240 \\
260 \\
280 \\
300\end{array}$ & $\begin{array}{l}32900 \\
35900 \\
43000 \\
39800 \\
16500\end{array}$ & $\begin{array}{l}1720 \\
1600 \\
1450 \\
1350 \\
1660\end{array}$ & $\begin{array}{r}1440 \\
1130 \\
900 \\
800 \\
641\end{array}$ & $\begin{array}{r}1510 \\
780 \\
530 \\
370 \\
320\end{array}$ & $\begin{array}{l}220 \\
240 \\
200 \\
170 \\
220\end{array}$ & $\begin{array}{l}4250 \\
2720 \\
2560 \\
5350 \\
4610\end{array}$ & $\begin{array}{l}140 \\
1 \leqslant 0 \\
140 \\
140 \\
140\end{array}$ \\
\hline $\begin{array}{l}26 \\
27 \\
28 \\
29 \\
30 \\
31\end{array}$ & $\begin{array}{l}550 \\
370 \\
270 \\
180 \\
180 \\
200\end{array}$ & $\begin{array}{r}1620 \\
1210 \\
1000 \\
870 \\
790 \\
=-0\end{array}$ & $\begin{array}{l}310 \\
310 \\
310 \\
290 \\
290 \\
290\end{array}$ & $\begin{array}{l}190 \\
190 \\
190 \\
190 \\
190 \\
190\end{array}$ & $\begin{array}{l}310 \\
320 \\
310 \\
--- \\
-2- \\
-\cdots\end{array}$ & $\begin{array}{r}12200 \\
7940 \\
7110 \\
7620 \\
20400 \\
31800\end{array}$ & $\begin{array}{r}4780 \\
5820 \\
3780 \\
2600 \\
8420 \\
-0 .\end{array}$ & $\begin{array}{l}600 \\
560 \\
530 \\
510 \\
800 \\
900\end{array}$ & $\begin{array}{r}270 \\
240 \\
238 \\
1560 \\
5300 \\
-0 .\end{array}$ & $\begin{array}{l}350 \\
310 \\
240 \\
200 \\
190 \\
280\end{array}$ & $\begin{array}{r}2400 \\
1760 \\
1580 \\
1260 \\
1000 \\
820\end{array}$ & $\begin{array}{l}140 \\
160 \\
140 \\
130 \\
140 \\
1\end{array}$ \\
\hline MEAN & 903 & 856 & 432 & 200 & 225 & 9001 & 5846 & 2107 & 1328 & 517 & 1932 & 261 \\
\hline WTR YR & & MEAN & 1980 & $\max$ & 43000 & & IN & 130 & & & & \\
\hline TOTAL & 27990 & 25690 & 13400 & 6190 & 6290 & 279040 & 175380 & 65311 & 39828 & 16020 & 59879 & 7830 \\
\hline
\end{tabular}

TOTAL LOAD FOR YEAR: 722848 POUNDS

TOTAL NITROGEN, WATER YEAR OCTOBER 1979 TO SEPTEMBER 1980

\begin{tabular}{|c|c|c|c|c|c|c|c|c|c|c|c|c|}
\hline \multicolumn{13}{|c|}{ MEAN VALUES } \\
\hline DAY & OCT & Nov & OEC & JAN & FEB & MAR & $A P R$ & MAY & JUN & JUL & AUG & SEP \\
\hline $\begin{array}{l}1 \\
2 \\
3 \\
4 \\
5\end{array}$ & $\begin{array}{l}150 \\
150 \\
160 \\
150 \\
160\end{array}$ & $\begin{array}{l}260 \\
300 \\
260 \\
250 \\
210\end{array}$ & $\begin{array}{l}300 \\
260 \\
250 \\
250 \\
250\end{array}$ & $\begin{array}{l}450 \\
380 \\
360 \\
320 \\
300\end{array}$ & $\begin{array}{l}490 \\
540 \\
560 \\
580 \\
580\end{array}$ & $\begin{array}{l}470 \\
390 \\
330 \\
290 \\
350\end{array}$ & $\begin{array}{r}590 \\
570 \\
830 \\
3790 \\
11800\end{array}$ & $\begin{array}{r}1110 \\
940 \\
750 \\
600 \\
510\end{array}$ & $\begin{array}{l}700 \\
580 \\
520 \\
500 \\
558\end{array}$ & $\begin{array}{l}160 \\
160 \\
160 \\
160 \\
150\end{array}$ & $\begin{array}{l}180 \\
160 \\
180 \\
200 \\
200\end{array}$ & $\begin{array}{l}710 \\
670 \\
600 \\
400 \\
360\end{array}$ \\
\hline $\begin{array}{r}6 \\
7 \\
8 \\
9 \\
10\end{array}$ & $\begin{array}{l}180 \\
180 \\
210 \\
250 \\
230\end{array}$ & $\begin{array}{l}230 \\
320 \\
320 \\
280 \\
250\end{array}$ & $\begin{array}{l}250 \\
260 \\
300 \\
540 \\
320\end{array}$ & $\begin{array}{l}280 \\
260 \\
250 \\
230 \\
210\end{array}$ & $\begin{array}{l}560 \\
540 \\
490 \\
410 \\
370\end{array}$ & $\begin{array}{l}370 \\
220 \\
220 \\
240 \\
240\end{array}$ & $\begin{array}{r}6660 \\
4860 \\
12600 \\
21800 \\
18900\end{array}$ & $\begin{array}{l}470 \\
400 \\
306 \\
360 \\
320\end{array}$ & $\begin{array}{r}6340 \\
6540 \\
11600 \\
7380 \\
8250\end{array}$ & $\begin{array}{l}180 \\
180 \\
160 \\
130 \\
130\end{array}$ & $\begin{array}{r}240 \\
223 \\
4380 \\
10300 \\
6430\end{array}$ & $\begin{array}{l}290 \\
260 \\
240 \\
260 \\
310\end{array}$ \\
\hline $\begin{array}{l}11 \\
12 \\
13 \\
14 \\
15\end{array}$ & $\begin{array}{l}210 \\
190 \\
230 \\
180 \\
160\end{array}$ & $\begin{array}{l}230 \\
250 \\
230 \\
230 \\
230\end{array}$ & $\begin{array}{l}260 \\
230 \\
120 \\
165 \\
160\end{array}$ & $\begin{array}{l}190 \\
190 \\
180 \\
180 \\
313\end{array}$ & $\begin{array}{l}350 \\
350 \\
350 \\
330 \\
310\end{array}$ & $\begin{array}{l}220 \\
180 \\
200 \\
220 \\
250\end{array}$ & $\begin{array}{r}10800 \\
5840 \\
4020 \\
2930 \\
3010\end{array}$ & $\begin{array}{r}340 \\
340 \\
360 \\
760 \\
1030\end{array}$ & $\begin{array}{r}2300 \\
866 \\
631 \\
730 \\
890\end{array}$ & $\begin{array}{l}130 \\
120 \\
120 \\
100 \\
130\end{array}$ & $\begin{array}{r}3390 \\
2120 \\
1340 \\
771 \\
485\end{array}$ & $\begin{array}{l}280 \\
260 \\
680 \\
880 \\
820\end{array}$ \\
\hline $\begin{array}{l}16 \\
17 \\
18 \\
19 \\
20\end{array}$ & $\begin{array}{l}190 \\
190 \\
190 \\
210 \\
430\end{array}$ & $\begin{array}{l}261 \\
260 \\
250 \\
250 \\
250\end{array}$ & $\begin{array}{l}150 \\
150 \\
150 \\
150 \\
160\end{array}$ & $\begin{array}{l}1660 \\
4860 \\
4280 \\
5140 \\
3300\end{array}$ & $\begin{array}{l}290 \\
250 \\
240 \\
250 \\
310\end{array}$ & $\begin{array}{r}350 \\
660 \\
1400 \\
5480 \\
5140\end{array}$ & $\begin{array}{l}7030 \\
5220 \\
3610 \\
2720 \\
2180\end{array}$ & $\begin{array}{r}990 \\
820 \\
960 \\
1450 \\
1330\end{array}$ & $\begin{array}{l}860 \\
550 \\
440 \\
500 \\
990\end{array}$ & $\begin{array}{r}270 \\
433 \\
280 \\
205 \\
6440\end{array}$ & $\begin{array}{r}408 \\
364 \\
373 \\
382 \\
1730\end{array}$ & $\begin{array}{r}522 \\
2040 \\
1820 \\
1150 \\
1610\end{array}$ \\
\hline $\begin{array}{l}21 \\
22 \\
23 \\
24 \\
25\end{array}$ & $\begin{array}{l}250 \\
230 \\
320 \\
500 \\
590\end{array}$ & $\begin{array}{l}260 \\
470 \\
670 \\
520 \\
380\end{array}$ & $\begin{array}{r}160 \\
180 \\
230 \\
320 \\
5160\end{array}$ & $\begin{array}{r}2230 \\
1560 \\
1100 \\
880 \\
745\end{array}$ & $\begin{array}{r}450 \\
670 \\
1190 \\
1080 \\
970\end{array}$ & $\begin{array}{r}2320 \\
1490 \\
998 \\
470 \\
500\end{array}$ & $\begin{array}{r}1750 \\
1480 \\
1230 \\
1000 \\
890\end{array}$ & $\begin{array}{l}890 \\
670 \\
550 \\
500 \\
440\end{array}$ & $\begin{array}{l}890 \\
550 \\
440 \\
320 \\
280\end{array}$ & $\begin{array}{r}10300 \\
4350 \\
2080 \\
640 \\
420\end{array}$ & $\begin{array}{r}3630 \\
2230 \\
1090 \\
622 \\
495\end{array}$ & $\begin{array}{l}3610 \\
15500 \\
17700 \\
17000 \\
10800\end{array}$ \\
\hline $\begin{array}{l}26 \\
27 \\
28 \\
29 \\
30 \\
31\end{array}$ & $\begin{array}{l}430 \\
300 \\
300 \\
300 \\
250 \\
250\end{array}$ & $\begin{array}{r}520 \\
1250 \\
1190 \\
670 \\
340 \\
---\end{array}$ & $\begin{array}{r}1140 \\
1250 \\
800 \\
670 \\
540 \\
500\end{array}$ & $\begin{array}{l}720 \\
630 \\
580 \\
510 \\
470 \\
470\end{array}$ & $\begin{array}{r}1030 \\
920 \\
600 \\
470 \\
0-0 \\
-0 .\end{array}$ & $\begin{array}{r}802 \\
1071 \\
1118 \\
950 \\
1050 \\
750\end{array}$ & $\begin{array}{r}800 \\
730 \\
700 \\
790 \\
1000 \\
-0-0\end{array}$ & $\begin{array}{l}390 \\
350 \\
320 \\
370 \\
730 \\
790\end{array}$ & $\begin{array}{l}260 \\
220 \\
220 \\
260 \\
240 \\
-\cdots\end{array}$ & $\begin{array}{l}350 \\
370 \\
300 \\
240 \\
180 \\
160\end{array}$ & $\begin{array}{r}667 \\
759 \\
673 \\
1370 \\
1150 \\
800\end{array}$ & $\begin{array}{r}5720 \\
4160 \\
3560 \\
2690 \\
2120 \\
-\cdots\end{array}$ \\
\hline MEAN & 249 & 380 & 603 & 1072 & 536 & 927 & 4671 & 650 & 1847 & 942 & 1527 & 3234 \\
\hline WTR YF & & MEAN & 1379 & $\operatorname{MAX}$ & 21800 & & In & 100 & & & & \\
\hline TOTAL & 7720 & 11391 & 18695 & 33228 & 15530 & 28739 & 140130 & 20146 & 55405 & 29188 & 47352 & 97022 \\
\hline
\end{tabular}

TOTAL LOAD FOR YEAR: 504546 POUNDS 
Table 26. Total nitrite plus nitrate nitrogen load, in pounds per day, for Onion River near Sheboygan Falls, 1979 and 1980 water years

TOTAL NITRITE PLUS NITKATE, WATER YEAR OCTOBER 1978 TO SEPTEMBER 1979

\begin{tabular}{|c|c|c|c|c|c|c|c|c|c|c|c|c|}
\hline DAY & OCT & NoV & DEC & JAN & FEB & MAR & APR & maY & JUN & JUL & AUG & SEP \\
\hline $\begin{array}{l}1 \\
2 \\
3 \\
4 \\
5\end{array}$ & $\begin{array}{r}1370 \\
1050 \\
690 \\
510 \\
380\end{array}$ & $\begin{array}{l}110 \\
120 \\
120 \\
120 \\
120\end{array}$ & $\begin{array}{l}520 \\
500 \\
490 \\
470 \\
470\end{array}$ & $\begin{array}{l}250 \\
250 \\
250 \\
240 \\
240\end{array}$ & $\begin{array}{l}180 \\
180 \\
180 \\
180 \\
180\end{array}$ & $\begin{array}{l}230 \\
240 \\
260 \\
320 \\
320\end{array}$ & $\begin{array}{r}14000 \\
8744 \\
5330 \\
4190 \\
5820\end{array}$ & $\begin{array}{l}7200 \\
5220 \\
9510 \\
4630 \\
3090\end{array}$ & $\begin{array}{l}160 \\
170 \\
120 \\
290 \\
180\end{array}$ & $\begin{array}{r}1939 \\
1087 \\
530 \\
170 \\
140\end{array}$ & $\begin{array}{l}60 \\
35 \\
26 \\
18 \\
18\end{array}$ & $\begin{array}{l}460 \\
420 \\
350 \\
270 \\
240\end{array}$ \\
\hline $\begin{array}{r}6 \\
7 \\
8 \\
9 \\
10\end{array}$ & $\begin{array}{r}1620 \\
1520 \\
1190 \\
970 \\
760\end{array}$ & $\begin{array}{l}120 \\
120 \\
120 \\
120 \\
120\end{array}$ & $\begin{array}{l}454 \\
440 \\
430 \\
430 \\
430\end{array}$ & $\begin{array}{l}230 \\
220 \\
220 \\
220 \\
220\end{array}$ & $\begin{array}{l}150 \\
160 \\
160 \\
160 \\
170\end{array}$ & $\begin{array}{l}310 \\
310 \\
310 \\
310 \\
310\end{array}$ & $\begin{array}{l}4470 \\
3470 \\
2370 \\
1970 \\
1930\end{array}$ & $\begin{array}{r}2240 \\
1530 \\
1060 \\
820 \\
640\end{array}$ & $\begin{array}{r}150 \\
1470 \\
690 \\
430 \\
6320\end{array}$ & $\begin{array}{r}100 \\
52 \\
35 \\
26 \\
18\end{array}$ & $\begin{array}{r}110 \\
88 \\
47 \\
74 \\
3410\end{array}$ & $\begin{array}{l}220 \\
190 \\
140 \\
120 \\
120\end{array}$ \\
\hline $\begin{array}{l}11 \\
12 \\
13 \\
14 \\
15\end{array}$ & $\begin{array}{l}620 \\
530 \\
420 \\
340 \\
280\end{array}$ & $\begin{array}{l}120 \\
120 \\
150 \\
210 \\
310\end{array}$ & $\begin{array}{l}410 \\
400 \\
380 \\
370 \\
360\end{array}$ & $\begin{array}{l}220 \\
220 \\
220 \\
150 \\
130\end{array}$ & $\begin{array}{l}170 \\
170 \\
160 \\
160 \\
160\end{array}$ & $\begin{array}{l}310 \\
320 \\
340 \\
350 \\
350\end{array}$ & $\begin{array}{l}2080 \\
7930 \\
9750 \\
7080 \\
4030\end{array}$ & $\begin{array}{l}530 \\
490 \\
420 \\
390 \\
460\end{array}$ & $\begin{array}{r}2260 \\
890 \\
490 \\
280 \\
200\end{array}$ & $\begin{array}{l}30 \\
52 \\
35 \\
85 \\
52\end{array}$ & $\begin{array}{r}5840 \\
2590 \\
754 \\
327 \\
214\end{array}$ & $\begin{array}{l}120 \\
120 \\
120 \\
120 \\
110\end{array}$ \\
\hline $\begin{array}{l}16 \\
17 \\
18 \\
19 \\
20\end{array}$ & $\begin{array}{l}310 \\
310 \\
300 \\
270 \\
280\end{array}$ & $\begin{array}{r}240 \\
550 \\
1620 \\
1370 \\
930\end{array}$ & $\begin{array}{l}360 \\
350 \\
340 \\
320 \\
320\end{array}$ & $\begin{array}{l}120 \\
130 \\
150 \\
170 \\
180\end{array}$ & $\begin{array}{l}150 \\
150 \\
150 \\
150 \\
150\end{array}$ & $\begin{array}{r}350 \\
350 \\
970 \\
3540 \\
6320\end{array}$ & $\begin{array}{l}2500 \\
1930 \\
1570 \\
1350 \\
1180\end{array}$ & $\begin{array}{r}330 \\
390 \\
490 \\
1230 \\
1120\end{array}$ & $\begin{array}{r}170 \\
130 \\
100 \\
95 \\
890\end{array}$ & $\begin{array}{r}22 \\
12 \\
8 \\
10 \\
16\end{array}$ & $\begin{array}{l}152 \\
124 \\
112 \\
104 \\
321\end{array}$ & $\begin{array}{l}99 \\
99 \\
90 \\
82 \\
74\end{array}$ \\
\hline $\begin{array}{l}21 \\
22 \\
23 \\
24 \\
25\end{array}$ & $\begin{array}{l}270 \\
210 \\
130 \\
270 \\
320\end{array}$ & $\begin{array}{r}650 \\
480 \\
730 \\
1620 \\
1230\end{array}$ & $\begin{array}{l}310 \\
310 \\
310 \\
300 \\
300\end{array}$ & $\begin{array}{l}190 \\
180 \\
180 \\
180 \\
180\end{array}$ & $\begin{array}{l}160 \\
170 \\
180 \\
180 \\
2170\end{array}$ & $\begin{array}{l}22700 \\
25300 \\
30500 \\
27400 \\
11000\end{array}$ & $\begin{array}{r}1080 \\
990 \\
900 \\
840 \\
1030\end{array}$ & $\begin{array}{l}730 \\
530 \\
390 \\
330 \\
261\end{array}$ & $\begin{array}{r}490 \\
140 \\
67 \\
34 \\
26\end{array}$ & $\begin{array}{r}12 \\
15 \\
10 \\
8 \\
12\end{array}$ & $\begin{array}{l}2140 \\
1360 \\
1110 \\
3010 \\
2220\end{array}$ & $\begin{array}{l}82 \\
82 \\
82 \\
82 \\
82\end{array}$ \\
\hline $\begin{array}{l}26 \\
27 \\
28 \\
29 \\
30 \\
31\end{array}$ & $\begin{array}{l}310 \\
210 \\
150 \\
100 \\
100 \\
110\end{array}$ & $\begin{array}{l}930 \\
690 \\
580 \\
500 \\
450 \\
--0\end{array}$ & $\begin{array}{l}270 \\
270 \\
270 \\
260 \\
260 \\
260\end{array}$ & $\begin{array}{l}170 \\
170 \\
170 \\
170 \\
160 \\
160\end{array}$ & $\begin{array}{l}200 \\
210 \\
224 \\
--- \\
---\end{array}$ & $\begin{array}{r}6650 \\
4960 \\
4290 \\
4470 \\
13700 \\
19200\end{array}$ & $\begin{array}{l}3050 \\
3740 \\
2410 \\
1640 \\
7780 \\
-\end{array}$ & $\begin{array}{l}230 \\
200 \\
190 \\
180 \\
330 \\
390\end{array}$ & $\begin{array}{r}18 \\
8 \\
8 \\
622 \\
3630 \\
-0-\end{array}$ & $\begin{array}{l}60 \\
46 \\
26 \\
18 \\
15 \\
40\end{array}$ & $\begin{array}{r}1070 \\
780 \\
740 \\
518 \\
540 \\
440\end{array}$ & $\begin{array}{r}82 \\
90 \\
82 \\
74 \\
82 \\
---\end{array}$ \\
\hline MEAN & 513 & 489 & 367 & 192 & 171 & 6009 & 3838 & 1469 & 684 & 151 & 915 & 146 \\
\hline WTR YR & 1979 & MFAN & 1254 & $\max$ & 30500 & & IN & 8 & & & & \\
\hline TOTAL & 15903 & 14670 & 11377 & 5952 & 4788 & 186279 & 115140 & 45539 & 20520 & 4681 & 28365 & 4380 \\
\hline
\end{tabular}

TOTAL NITRITE PLUS NITRATE. WATER YEAR OCTOBER 1979 TO SEPTEMBER 1980

\begin{tabular}{|c|c|c|c|c|c|c|c|c|c|c|c|c|}
\hline DAY & OCT & Nov & DEC & JAN & FEB & MAR & APR & MAY & JUN & JUL & AUG & SEP \\
\hline $\begin{array}{l}1 \\
2 \\
3 \\
4 \\
5\end{array}$ & $\begin{array}{l}90 \\
90 \\
99 \\
90 \\
99\end{array}$ & $\begin{array}{l}150 \\
170 \\
150 \\
140 \\
120\end{array}$ & $\begin{array}{l}170 \\
150 \\
140 \\
140 \\
140\end{array}$ & $\begin{array}{l}250 \\
220 \\
200 \\
180 \\
170\end{array}$ & $\begin{array}{l}260 \\
280 \\
280 \\
290 \\
290\end{array}$ & $\begin{array}{l}300 \\
260 \\
230 \\
210 \\
240\end{array}$ & $\begin{array}{r}370 \\
350 \\
400 \\
2060 \\
8500\end{array}$ & $\begin{array}{l}49 \\
35 \\
22 \\
14 \\
10\end{array}$ & $\begin{array}{r}220 \\
160 \\
140 \\
130 \\
74\end{array}$ & $\begin{array}{l}24 \\
24 \\
24 \\
24 \\
21\end{array}$ & $\begin{array}{l}28 \\
24 \\
28 \\
37 \\
33\end{array}$ & $\begin{array}{r}170 \\
150 \\
130 \\
70 \\
60\end{array}$ \\
\hline $\begin{array}{r}6 \\
7 \\
8 \\
9 \\
10\end{array}$ & $\begin{array}{l}110 \\
110 \\
120 \\
140 \\
130\end{array}$ & $\begin{array}{l}130 \\
180 \\
180 \\
160 \\
140\end{array}$ & $\begin{array}{l}140 \\
150 \\
170 \\
290 \\
180\end{array}$ & $\begin{array}{l}160 \\
150 \\
140 \\
130 \\
120\end{array}$ & $\begin{array}{l}280 \\
280 \\
260 \\
230 \\
210\end{array}$ & $\begin{array}{l}240 \\
160 \\
160 \\
170 \\
170\end{array}$ & $\begin{array}{r}5300 \\
3980 \\
9570 \\
16100 \\
14400\end{array}$ & $\begin{array}{l}9 \\
6 \\
4 \\
5 \\
4\end{array}$ & $\begin{array}{l}3830 \\
2550 \\
7760 \\
4060 \\
5600\end{array}$ & $\begin{array}{l}28 \\
28 \\
24 \\
17 \\
17\end{array}$ & $\begin{array}{r}43 \\
43 \\
1674 \\
5482 \\
3952\end{array}$ & $\begin{array}{l}44 \\
37 \\
34 \\
37 \\
48\end{array}$ \\
\hline $\begin{array}{l}11 \\
12 \\
13 \\
14 \\
15\end{array}$ & $\begin{array}{r}120 \\
120 \\
130 \\
110 \\
99\end{array}$ & $\begin{array}{l}130 \\
140 \\
130 \\
130 \\
130\end{array}$ & $\begin{array}{l}150 \\
13 n \\
120 \\
110 \\
99\end{array}$ & $\begin{array}{l}120 \\
120 \\
110 \\
110 \\
240\end{array}$ & $\begin{array}{l}200 \\
200 \\
200 \\
190 \\
190\end{array}$ & $\begin{array}{l}160 \\
140 \\
150 \\
160 \\
170\end{array}$ & $\begin{array}{r}8080 \\
1340 \\
630 \\
340 \\
360\end{array}$ & $\begin{array}{r}5 \\
5 \\
5 \\
250 \\
400\end{array}$ & $\begin{array}{r}1380 \\
407 \\
278 \\
230 \\
320\end{array}$ & $\begin{array}{l}17 \\
14 \\
14 \\
12 \\
17\end{array}$ & $\begin{array}{r}1983 \\
986 \\
469 \\
202 \\
99\end{array}$ & $\begin{array}{r}40 \\
37 \\
160 \\
230 \\
210\end{array}$ \\
\hline $\begin{array}{l}16 \\
17 \\
18 \\
19 \\
20\end{array}$ & $\begin{array}{l}120 \\
120 \\
120 \\
120 \\
240\end{array}$ & $\begin{array}{l}147 \\
150 \\
140 \\
140 \\
140\end{array}$ & $\begin{array}{l}90 \\
90 \\
90 \\
90 \\
99\end{array}$ & $\begin{array}{r}755 \\
3310 \\
3640 \\
3340 \\
2030\end{array}$ & $\begin{array}{l}170 \\
150 \\
140 \\
150 \\
170\end{array}$ & $\begin{array}{r}220 \\
480 \\
530 \\
1940 \\
1900\end{array}$ & $\begin{array}{r}1940 \\
1070 \\
510 \\
290 \\
190\end{array}$ & $\begin{array}{l}370 \\
280 \\
350 \\
670 \\
580\end{array}$ & $\begin{array}{l}300 \\
150 \\
110 \\
130 \\
370\end{array}$ & $\begin{array}{r}54 \\
119 \\
55 \\
40 \\
3210\end{array}$ & $\begin{array}{r}96 \\
75 \\
80 \\
80 \\
613\end{array}$ & $\begin{array}{r}160 \\
1110 \\
992 \\
592 \\
735\end{array}$ \\
\hline $\begin{array}{l}21 \\
22 \\
23 \\
24 \\
25\end{array}$ & $\begin{array}{l}140 \\
130 \\
183 \\
270 \\
320\end{array}$ & $\begin{array}{l}150 \\
260 \\
350 \\
280 \\
220\end{array}$ & $\begin{array}{r}99 \\
110 \\
130 \\
180 \\
2220\end{array}$ & $\begin{array}{r}1440 \\
1050 \\
780 \\
640 \\
420\end{array}$ & $\begin{array}{l}230 \\
320 \\
310 \\
700 \\
590\end{array}$ & $\begin{array}{l}960 \\
660 \\
480 \\
280 \\
300\end{array}$ & $\begin{array}{r}120 \\
86 \\
60 \\
39 \\
31\end{array}$ & $\begin{array}{l}320 \\
200 \\
150 \\
130 \\
110\end{array}$ & $\begin{array}{r}320 \\
150 \\
110 \\
68 \\
54\end{array}$ & $\begin{array}{r}6620 \\
2580 \\
580 \\
190 \\
100\end{array}$ & $\begin{array}{r}1840 \\
890 \\
310 \\
140 \\
96\end{array}$ & $\begin{array}{r}1560 \\
7520 \\
10500 \\
10600 \\
6890\end{array}$ \\
\hline $\begin{array}{l}26 \\
27 \\
28 \\
29 \\
30 \\
31\end{array}$ & $\begin{array}{l}240 \\
170 \\
170 \\
170 \\
140 \\
140\end{array}$ & $\begin{array}{l}280 \\
620 \\
590 \\
350 \\
190 \\
--.\end{array}$ & $\begin{array}{r}1820 \\
620 \\
420 \\
350 \\
290 \\
270\end{array}$ & $\begin{array}{l}450 \\
370 \\
330 \\
280 \\
270 \\
270\end{array}$ & $\begin{array}{l}640 \\
570 \\
400 \\
340 \\
-\square \\
---\end{array}$ & $\begin{array}{l}490 \\
709 \\
950 \\
700 \\
750 \\
500\end{array}$ & $\begin{array}{r}25 \\
21 \\
19 \\
24 \\
39 \\
---\end{array}$ & $\begin{array}{r}92 \\
75 \\
68 \\
83 \\
230 \\
260\end{array}$ & $\begin{array}{r}48 \\
37 \\
37 \\
48 \\
43 \\
---\end{array}$ & $\begin{array}{l}75 \\
83 \\
61 \\
43 \\
25 \\
24\end{array}$ & $\begin{array}{l}150 \\
180 \\
197 \\
430 \\
320 \\
190\end{array}$ & $\begin{array}{r}3820 \\
2800 \\
2360 \\
1700 \\
1350 \\
-\end{array}$ \\
\hline MEAN & 144 & 206 & 298 & 703 & 311 & 476 & 2541 & 155 & 970 & 457 & 670 & 1805 \\
\hline WTR YR & & & 723 & $\max$ & 16100 & & & 4 & & & & \\
\hline TOTAL & 4464 & 6180 & 9238 & 21793 & 9019 & 14756 & 76230 & 4805 & 29100 & 14167 & 20770 & 54150 \\
\hline
\end{tabular}

TOTAL LOAD FOR YEAR: 264672 POUNDS 
Table 27. Total ammonia nitrogen load, in pounds per day, for Onion River near Sheboygan Falls, 1979 and 1980 water years

TOTAL AMMONIA NITROGEN, WATER YEAR OCTOBER 1978 TO SEPTEMBER 1979

\begin{tabular}{|c|c|c|c|c|c|c|c|c|c|c|c|c|}
\hline DAY & OCT & NoV & DEC & JAN & FEB & MAR & APR & MAY & JUN & JUL & AUG & SEP \\
\hline $\begin{array}{l}1 \\
2 \\
3 \\
4 \\
5\end{array}$ & $\begin{array}{l}64 \\
53 \\
40 \\
33 \\
27\end{array}$ & $\begin{array}{l}12 \\
12 \\
12 \\
12 \\
12\end{array}$ & $\begin{array}{l}27 \\
26 \\
26 \\
24 \\
24\end{array}$ & $\begin{array}{l}18 \\
18 \\
18 \\
17 \\
17\end{array}$ & $\begin{array}{l}14 \\
14 \\
14 \\
14 \\
14\end{array}$ & $\begin{array}{l}44 \\
46 \\
50 \\
61 \\
61\end{array}$ & $\begin{array}{r}1140 \\
494 \\
300 \\
290 \\
330\end{array}$ & $\begin{array}{r}130 \\
100 \\
150 \\
95 \\
73\end{array}$ & $\begin{array}{r}11 \\
11 \\
9 \\
16 \\
12\end{array}$ & $\begin{array}{l}72 \\
62 \\
53 \\
39 \\
37\end{array}$ & $\begin{array}{l}17 \\
14 \\
13 \\
11 \\
11\end{array}$ & $\begin{array}{l}33 \\
31 \\
27 \\
22 \\
20\end{array}$ \\
\hline $\begin{array}{r}6 \\
7 \\
8 \\
9 \\
10\end{array}$ & $\begin{array}{l}71 \\
68 \\
58 \\
50 \\
43\end{array}$ & $\begin{array}{l}12 \\
12 \\
12 \\
12 \\
12\end{array}$ & $\begin{array}{l}23 \\
23 \\
22 \\
22 \\
22\end{array}$ & $\begin{array}{l}17 \\
16 \\
16 \\
16 \\
16\end{array}$ & $\begin{array}{l}14 \\
14 \\
14 \\
15 \\
16\end{array}$ & $\begin{array}{l}59 \\
59 \\
59 \\
59 \\
59\end{array}$ & $\begin{array}{r}240 \\
170 \\
110 \\
85 \\
83\end{array}$ & $\begin{array}{l}60 \\
47 \\
37 \\
31 \\
27\end{array}$ & $\begin{array}{r}10 \\
70 \\
60 \\
50 \\
100\end{array}$ & $\begin{array}{l}34 \\
28 \\
25 \\
23 \\
21\end{array}$ & $\begin{array}{r}18 \\
16 \\
12 \\
12 \\
178\end{array}$ & $\begin{array}{l}19 \\
17 \\
14 \\
12 \\
11\end{array}$ \\
\hline $\begin{array}{l}11 \\
12 \\
13 \\
14 \\
15\end{array}$ & $\begin{array}{l}37 \\
34 \\
28 \\
25 \\
22\end{array}$ & $\begin{array}{l}12 \\
12 \\
14 \\
18 \\
23\end{array}$ & $\begin{array}{l}22 \\
21 \\
21 \\
20 \\
19\end{array}$ & $\begin{array}{l}16 \\
16 \\
16 \\
12 \\
10\end{array}$ & $\begin{array}{l}16 \\
16 \\
16 \\
16 \\
16\end{array}$ & $\begin{array}{l}59 \\
62 \\
66 \\
68 \\
68\end{array}$ & $\begin{array}{r}91 \\
490 \\
640 \\
420 \\
210\end{array}$ & $\begin{array}{l}24 \\
22 \\
20 \\
19 \\
21\end{array}$ & $\begin{array}{l}79 \\
61 \\
52 \\
45 \\
41\end{array}$ & $\begin{array}{l}24 \\
28 \\
25 \\
32 \\
28\end{array}$ & $\begin{array}{r}233 \\
172 \\
78 \\
51 \\
36\end{array}$ & $\begin{array}{l}11 \\
12 \\
12 \\
11 \\
11\end{array}$ \\
\hline $\begin{array}{l}16 \\
17 \\
18 \\
19 \\
20\end{array}$ & $\begin{array}{l}23 \\
23 \\
23 \\
21 \\
22\end{array}$ & $\begin{array}{l}20 \\
34 \\
71 \\
64 \\
49\end{array}$ & $\begin{array}{l}19 \\
18 \\
18 \\
18 \\
18\end{array}$ & $\begin{array}{l}10 \\
10 \\
12 \\
14 \\
14\end{array}$ & $\begin{array}{l}15 \\
15 \\
16 \\
16 \\
16\end{array}$ & $\begin{array}{r}68 \\
68 \\
120 \\
370 \\
480\end{array}$ & $\begin{array}{r}110 \\
83 \\
64 \\
52 \\
44\end{array}$ & $\begin{array}{l}17 \\
19 \\
22 \\
40 \\
38\end{array}$ & $\begin{array}{l}39 \\
36 \\
34 \\
33 \\
61\end{array}$ & $\begin{array}{l}22 \\
19 \\
17 \\
18 \\
18\end{array}$ & $\begin{array}{l}26 \\
21 \\
20 \\
18 \\
31\end{array}$ & $\begin{array}{r}10 \\
10 \\
9 \\
9 \\
8\end{array}$ \\
\hline $\begin{array}{l}21 \\
22 \\
23 \\
24 \\
25\end{array}$ & $\begin{array}{l}21 \\
18 \\
13 \\
21 \\
24\end{array}$ & $\begin{array}{l}38 \\
31 \\
41 \\
71 \\
59\end{array}$ & $\begin{array}{l}17 \\
17 \\
17 \\
16 \\
16\end{array}$ & $\begin{array}{l}15 \\
15 \\
15 \\
15 \\
15\end{array}$ & $\begin{array}{l}17 \\
18 \\
19 \\
28 \\
30\end{array}$ & $\begin{array}{r}1540 \\
1580 \\
1990 \\
2530 \\
982\end{array}$ & $\begin{array}{l}39 \\
36 \\
32 \\
29 \\
38\end{array}$ & $\begin{array}{l}29 \\
24 \\
19 \\
17 \\
15\end{array}$ & $\begin{array}{l}52 \\
37 \\
30 \\
25 \\
23\end{array}$ & $\begin{array}{l}19 \\
20 \\
18 \\
17 \\
19\end{array}$ & $\begin{array}{r}91 \\
59 \\
67 \\
150 \\
120\end{array}$ & $\begin{array}{l}9 \\
9 \\
9 \\
9 \\
9\end{array}$ \\
\hline $\begin{array}{l}26 \\
27 \\
28 \\
29 \\
30 \\
31\end{array}$ & $\begin{array}{l}23 \\
18 \\
14 \\
11 \\
11 \\
12\end{array}$ & $\begin{array}{r}49 \\
40 \\
36 \\
32 \\
30 \\
-\end{array}$ & $\begin{array}{l}16 \\
16 \\
16 \\
15 \\
15 \\
15\end{array}$ & $\begin{array}{l}14 \\
14 \\
14 \\
14 \\
14 \\
14\end{array}$ & $\begin{array}{r}31 \\
32 \\
43 \\
-0- \\
-0- \\
-\infty-0\end{array}$ & $\begin{array}{r}550 \\
380 \\
300 \\
291 \\
1080 \\
1890\end{array}$ & $\begin{array}{r}150 \\
190 \\
110 \\
67 \\
130 \\
-\end{array}$ & $\begin{array}{l}14 \\
13 \\
12 \\
12 \\
17 \\
19\end{array}$ & $\begin{array}{r}21 \\
20 \\
20 \\
40 \\
112 \\
-\end{array}$ & $\begin{array}{l}17 \\
16 \\
13 \\
11 \\
11 \\
15\end{array}$ & $\begin{array}{l}66 \\
51 \\
49 \\
26 \\
38 \\
32\end{array}$ & $\begin{array}{r}9 \\
9 \\
9 \\
9 \\
-9\end{array}$ \\
\hline$\underset{\operatorname{MAX}}{\operatorname{MAN}}$ & $\begin{array}{l}71 \\
11\end{array}$ & $\begin{array}{l}71 \\
12\end{array}$ & $\begin{array}{l}27 \\
15\end{array}$ & $\begin{array}{l}18 \\
10\end{array}$ & $\begin{array}{l}43 \\
14\end{array}$ & $\begin{array}{r}2530 \\
44\end{array}$ & $\begin{array}{r}1140 \\
29\end{array}$ & $\begin{array}{r}150 \\
12\end{array}$ & $\begin{array}{l}112 \\
9.0\end{array}$ & $\begin{array}{l}72 \\
11\end{array}$ & $\begin{array}{r}233 \\
11\end{array}$ & $\begin{array}{r}33 \\
8.0\end{array}$ \\
\hline WTR YR 1979 & $\max$ & 2530 & MIN 8.0 & & & & & & & & & \\
\hline TOTAL & 951 & 864 & 608 & 458 & 519 & 15099 & 6267 & 1183 & 3210 & 801 & 1737 & 389 \\
\hline
\end{tabular}

TOTAL AMMONIA NITROGEN, WATER YEAR OCTOBER 1979 TO SEPTEMBER 1980

\begin{tabular}{|c|c|c|c|c|c|c|c|c|c|c|c|c|}
\hline DAY & OCT & NOV & DEC & JAN & FEB & MAR & APR & MAY & JUN & JUL & AUG & SEP \\
\hline $\begin{array}{l}1 \\
2 \\
3 \\
4 \\
5\end{array}$ & $\begin{array}{l}10 \\
10 \\
10 \\
10 \\
10\end{array}$ & $\begin{array}{l}14 \\
16 \\
14 \\
14 \\
12\end{array}$ & $\begin{array}{l}16 \\
14 \\
14 \\
14 \\
14\end{array}$ & $\begin{array}{l}20 \\
18 \\
18 \\
16 \\
16\end{array}$ & $\begin{array}{l}26 \\
28 \\
28 \\
29 \\
29\end{array}$ & $\begin{array}{l}25 \\
22 \\
19 \\
18 \\
20\end{array}$ & $\begin{array}{r}60 \\
60 \\
60 \\
108 \\
274\end{array}$ & $\begin{array}{l}69 \\
63 \\
57 \\
51 \\
47\end{array}$ & $\begin{array}{l}50 \\
46 \\
44 \\
43 \\
50\end{array}$ & $\begin{array}{l}26 \\
26 \\
26 \\
26 \\
25\end{array}$ & $\begin{array}{l}3 \\
3 \\
3 \\
3 \\
3\end{array}$ & $\begin{array}{r}18 \\
17 \\
15 \\
9 \\
8\end{array}$ \\
\hline $\begin{array}{r}6 \\
7 \\
8 \\
9 \\
10\end{array}$ & $\begin{array}{l}11 \\
11 \\
12 \\
14 \\
13\end{array}$ & $\begin{array}{l}13 \\
16 \\
16 \\
15 \\
14\end{array}$ & $\begin{array}{l}14 \\
14 \\
16 \\
23 \\
16\end{array}$ & $\begin{array}{l}15 \\
14 \\
14 \\
13 \\
12\end{array}$ & $\begin{array}{l}28 \\
28 \\
26 \\
23 \\
21\end{array}$ & $\begin{array}{l}21 \\
14 \\
14 \\
15 \\
15\end{array}$ & $\begin{array}{l}154 \\
131 \\
238 \\
333 \\
252\end{array}$ & $\begin{array}{l}45 \\
42 \\
52 \\
40 \\
37\end{array}$ & $\begin{array}{l}122 \\
194 \\
175 \\
137 \\
152\end{array}$ & $\begin{array}{l}27 \\
27 \\
26 \\
23 \\
23\end{array}$ & $\begin{array}{r}4 \\
191 \\
354 \\
159\end{array}$ & $\begin{array}{l}7 \\
6 \\
5 \\
6 \\
7\end{array}$ \\
\hline $\begin{array}{l}11 \\
12 \\
13 \\
14 \\
15\end{array}$ & $\begin{array}{l}12 \\
12 \\
13 \\
11 \\
10\end{array}$ & $\begin{array}{l}13 \\
14 \\
13 \\
13 \\
13\end{array}$ & $\begin{array}{r}11 \\
13 \\
12 \\
7 \\
10\end{array}$ & $\begin{array}{l}12 \\
12 \\
11 \\
11 \\
20\end{array}$ & $\begin{array}{l}20 \\
20 \\
20 \\
19 \\
19\end{array}$ & $\begin{array}{l}14 \\
13 \\
14 \\
14 \\
16\end{array}$ & $\begin{array}{l}220 \\
160 \\
130 \\
110 \\
110\end{array}$ & $\begin{array}{l}38 \\
38 \\
40 \\
52 \\
59\end{array}$ & $\begin{array}{l}66 \\
43 \\
53 \\
51 \\
56\end{array}$ & $\begin{array}{l}23 \\
22 \\
22 \\
21 \\
23\end{array}$ & $\begin{array}{l}90 \\
63 \\
45 \\
28 \\
20\end{array}$ & $\begin{array}{r}6 \\
6 \\
11 \\
15 \\
14\end{array}$ \\
\hline $\begin{array}{l}16 \\
17 \\
18 \\
19 \\
20\end{array}$ & $\begin{array}{l}12 \\
12 \\
12 \\
12 \\
20\end{array}$ & $\begin{array}{l}17 \\
14 \\
14 \\
14 \\
14\end{array}$ & $\begin{array}{l}10 \\
10 \\
10 \\
10 \\
10\end{array}$ & $\begin{array}{r}84 \\
350 \\
280 \\
350 \\
210\end{array}$ & $\begin{array}{l}18 \\
16 \\
15 \\
16 \\
25\end{array}$ & $\begin{array}{r}20 \\
45 \\
320 \\
1280 \\
936\end{array}$ & $\begin{array}{r}170 \\
150 \\
120 \\
110 \\
96\end{array}$ & $\begin{array}{l}58 \\
54 \\
58 \\
70 \\
67\end{array}$ & $\begin{array}{l}55 \\
45 \\
41 \\
43 \\
58\end{array}$ & $\begin{array}{r}5 \\
4 \\
7 \\
133\end{array}$ & $\begin{array}{l}12 \\
10 \\
10 \\
10 \\
50\end{array}$ & $\begin{array}{l}11 \\
79 \\
31 \\
18 \\
30\end{array}$ \\
\hline $\begin{array}{l}21 \\
22 \\
23 \\
24 \\
25\end{array}$ & $\begin{array}{l}14 \\
13 \\
16 \\
22 \\
25\end{array}$ & $\begin{array}{l}14 \\
21 \\
27 \\
23 \\
18\end{array}$ & $\begin{array}{l}10 \\
11 \\
13 \\
16 \\
110\end{array}$ & $\begin{array}{r}130 \\
88 \\
57 \\
44 \\
36\end{array}$ & $\begin{array}{l}30 \\
45 \\
80 \\
75 \\
65\end{array}$ & $\begin{array}{r}350 \\
210 \\
130 \\
110 \\
90\end{array}$ & $\begin{array}{l}86 \\
79 \\
72 \\
65 \\
61\end{array}$ & $\begin{array}{l}56 \\
49 \\
45 \\
43 \\
41\end{array}$ & $\begin{array}{l}56 \\
45 \\
41 \\
35 \\
33\end{array}$ & $\begin{array}{r}209 \\
84 \\
25 \\
11 \\
7\end{array}$ & $\begin{array}{r}120 \\
66 \\
29 \\
16 \\
12\end{array}$ & $\begin{array}{l}168 \\
356 \\
470 \\
443 \\
267\end{array}$ \\
\hline $\begin{array}{l}26 \\
27 \\
28 \\
29 \\
30 \\
31\end{array}$ & $\begin{array}{l}20 \\
16 \\
16 \\
16 \\
14 \\
14\end{array}$ & $\begin{array}{r}23 \\
41 \\
40 \\
27 \\
17 \\
-\end{array}$ & $\begin{array}{l}92 \\
41 \\
30 \\
27 \\
23 \\
22\end{array}$ & $\begin{array}{l}34 \\
31 \\
29 \\
27 \\
25 \\
25\end{array}$ & $\begin{array}{r}70 \\
65 \\
40 \\
35 \\
--0 \\
-\end{array}$ & $\begin{array}{l}80 \\
99 \\
85 \\
80 \\
85 \\
70\end{array}$ & $\begin{array}{r}58 \\
56 \\
55 \\
58 \\
65 \\
-\end{array}$ & $\begin{array}{l}38 \\
36 \\
35 \\
37 \\
51 \\
53\end{array}$ & $\begin{array}{r}32 \\
30 \\
30 \\
32 \\
31 \\
-\end{array}$ & $\begin{array}{l}6 \\
6 \\
5 \\
4 \\
3 \\
3\end{array}$ & $\begin{array}{r}17 \\
20 \\
9 \\
38 \\
30 \\
20\end{array}$ & $\begin{array}{r}137 \\
94 \\
73 \\
48 \\
44 \\
\end{array}$ \\
\hline $\begin{array}{l}\operatorname{MAX} \\
\operatorname{MIN}\end{array}$ & $\begin{array}{l}25 \\
10\end{array}$ & $\begin{array}{l}41 \\
12\end{array}$ & $\begin{array}{l}110 \\
7.0\end{array}$ & $\begin{array}{r}350 \\
11\end{array}$ & $\begin{array}{l}80 \\
15\end{array}$ & $\begin{array}{r}1280 \\
13\end{array}$ & $\begin{array}{r}333 \\
55\end{array}$ & $\begin{array}{l}70 \\
35\end{array}$ & $\begin{array}{r}194 \\
30\end{array}$ & $\begin{array}{l}209 \\
3.0\end{array}$ & $\begin{array}{l}354 \\
3.0\end{array}$ & $\begin{array}{l}470 \\
5.0\end{array}$ \\
\hline
\end{tabular}

WTR YR 1980 MAX 1280 MIN 3.0

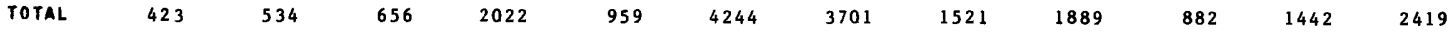
TOTAL LOAO FOR YEAR: 20692 POUMDS 
Table 28. Total phosphorus load, in pounds per day, for Onion River near Sheboygan Falls, 1979 and 1980 water years

TOTAL PHOSPHORUS, WATER YEAR OCTOBER 1978 TO SEPTEMBER 1979

\begin{tabular}{|c|c|c|c|c|c|c|c|c|c|c|c|c|}
\hline DAY & OCT & NOV & DEC & JAN & FEB & MAR & APR & MAY & JUN & JUL & AUG & SEP \\
\hline $\begin{array}{l}1 \\
2 \\
3 \\
4 \\
5\end{array}$ & $\begin{array}{r}200 \\
150 \\
99 \\
74 \\
50\end{array}$ & $\begin{array}{l}16 \\
17 \\
17 \\
17 \\
17\end{array}$ & $\begin{array}{l}55 \\
53 \\
51 \\
47 \\
47\end{array}$ & $\begin{array}{l}21 \\
21 \\
21 \\
20 \\
20\end{array}$ & $\begin{array}{l}14 \\
14 \\
14 \\
14 \\
14\end{array}$ & $\begin{array}{l}41 \\
43 \\
49 \\
67 \\
67\end{array}$ & $\begin{array}{r}1510 \\
610 \\
450 \\
440 \\
490\end{array}$ & $\begin{array}{l}390 \\
320 \\
470 \\
290 \\
220\end{array}$ & $\begin{array}{l}31 \\
33 \\
26 \\
46 \\
34\end{array}$ & $\begin{array}{r}206 \\
200 \\
150 \\
96 \\
89\end{array}$ & $\begin{array}{l}65 \\
53 \\
47 \\
41 \\
41\end{array}$ & $\begin{array}{r}120 \\
110 \\
98 \\
79 \\
70\end{array}$ \\
\hline $\begin{array}{r}6 \\
7 \\
8 \\
9 \\
10\end{array}$ & $\begin{array}{l}240 \\
220 \\
170 \\
140 \\
110\end{array}$ & $\begin{array}{l}17 \\
17 \\
17 \\
17 \\
17\end{array}$ & $\begin{array}{l}45 \\
43 \\
41 \\
41 \\
41\end{array}$ & $\begin{array}{l}20 \\
18 \\
18 \\
18 \\
18\end{array}$ & $\begin{array}{l}14 \\
16 \\
16 \\
17 \\
18\end{array}$ & $\begin{array}{l}64 \\
64 \\
64 \\
64 \\
64\end{array}$ & $\begin{array}{l}370 \\
290 \\
200 \\
170 \\
160\end{array}$ & $\begin{array}{r}180 \\
140 \\
110 \\
92 \\
79\end{array}$ & $\begin{array}{r}30 \\
220 \\
170 \\
140 \\
370\end{array}$ & $\begin{array}{l}80 \\
62 \\
53 \\
47 \\
41\end{array}$ & $\begin{array}{r}71 \\
57 \\
44 \\
48 \\
886\end{array}$ & $\begin{array}{l}68 \\
60 \\
47 \\
41 \\
39\end{array}$ \\
\hline $\begin{array}{l}11 \\
12 \\
13 \\
14 \\
15\end{array}$ & $\begin{array}{l}89 \\
76 \\
60 \\
49 \\
41\end{array}$ & $\begin{array}{l}17 \\
17 \\
21 \\
30 \\
45\end{array}$ & $\begin{array}{l}41 \\
39 \\
37 \\
35 \\
33\end{array}$ & $\begin{array}{r}18 \\
18 \\
18 \\
12 \\
9\end{array}$ & $\begin{array}{l}18 \\
18 \\
18 \\
18 \\
18\end{array}$ & $\begin{array}{l}64 \\
69 \\
76 \\
79 \\
79\end{array}$ & $\begin{array}{l}180 \\
660 \\
810 \\
590 \\
340\end{array}$ & $\begin{array}{l}69 \\
66 \\
60 \\
56 \\
63\end{array}$ & $\begin{array}{l}350 \\
180 \\
140 \\
120 \\
100\end{array}$ & $\begin{array}{l}50 \\
62 \\
53 \\
74 \\
62\end{array}$ & $\begin{array}{r}1050 \\
541 \\
234 \\
156 \\
114\end{array}$ & $\begin{array}{l}39 \\
41 \\
41 \\
39 \\
36\end{array}$ \\
\hline $\begin{array}{l}16 \\
17 \\
18 \\
19 \\
20\end{array}$ & $\begin{array}{l}45 \\
45 \\
43 \\
39 \\
41\end{array}$ & $\begin{array}{r}35 \\
79 \\
240 \\
200 \\
130\end{array}$ & $\begin{array}{l}33 \\
31 \\
30 \\
30 \\
30\end{array}$ & $\begin{array}{r}8 \\
9 \\
12 \\
14 \\
16\end{array}$ & $\begin{array}{l}17 \\
17 \\
18 \\
18 \\
18\end{array}$ & $\begin{array}{r}79 \\
79 \\
80 \\
290 \\
490\end{array}$ & $\begin{array}{l}210 \\
160 \\
130 \\
110 \\
100\end{array}$ & $\begin{array}{r}50 \\
57 \\
66 \\
120 \\
110\end{array}$ & $\begin{array}{r}96 \\
86 \\
80 \\
77 \\
180\end{array}$ & $\begin{array}{l}44 \\
36 \\
30 \\
33 \\
33\end{array}$ & $\begin{array}{r}85 \\
73 \\
69 \\
66 \\
132\end{array}$ & $\begin{array}{l}34 \\
34 \\
31 \\
29 \\
27\end{array}$ \\
\hline $\begin{array}{l}21 \\
22 \\
23 \\
24 \\
25\end{array}$ & $\begin{array}{l}39 \\
30 \\
18 \\
39 \\
47\end{array}$ & $\begin{array}{r}94 \\
69 \\
100 \\
240 \\
180\end{array}$ & $\begin{array}{l}28 \\
28 \\
28 \\
26 \\
26\end{array}$ & $\begin{array}{l}17 \\
17 \\
17 \\
17 \\
17\end{array}$ & $\begin{array}{l}19 \\
21 \\
25 \\
28 \\
31\end{array}$ & $\begin{array}{r}1680 \\
1860 \\
2240 \\
1960 \\
799\end{array}$ & $\begin{array}{l}91 \\
84 \\
77 \\
71 \\
88\end{array}$ & $\begin{array}{l}85 \\
69 \\
56 \\
51 \\
45\end{array}$ & $\begin{array}{r}140 \\
89 \\
68 \\
53 \\
47\end{array}$ & $\begin{array}{l}36 \\
39 \\
33 \\
30 \\
36\end{array}$ & $\begin{array}{l}474 \\
252 \\
260 \\
590 \\
490\end{array}$ & $\begin{array}{l}29 \\
29 \\
29 \\
29 \\
29\end{array}$ \\
\hline $\begin{array}{l}26 \\
27 \\
28 \\
29 \\
30 \\
31\end{array}$ & $\begin{array}{l}45 \\
30 \\
21 \\
14 \\
14 \\
16\end{array}$ & $\begin{array}{r}130 \\
99 \\
84 \\
71 \\
64 \\
-0-\end{array}$ & $\begin{array}{l}25 \\
25 \\
25 \\
23 \\
23 \\
23\end{array}$ & $\begin{array}{l}16 \\
16 \\
16 \\
16 \\
16 \\
16\end{array}$ & $\begin{array}{r}33 \\
35 \\
36 \\
--0 \\
--\infty \\
--\infty\end{array}$ & $\begin{array}{r}480 \\
360 \\
320 \\
296 \\
1160 \\
2930\end{array}$ & $\begin{array}{l}260 \\
310 \\
200 \\
140 \\
410 \\
-\cdots\end{array}$ & $\begin{array}{l}39 \\
37 \\
35 \\
34 \\
51 \\
56\end{array}$ & $\begin{array}{r}41 \\
39 \\
39 \\
151 \\
332 \\
---\end{array}$ & $\begin{array}{l}65 \\
59 \\
47 \\
41 \\
39 \\
56\end{array}$ & $\begin{array}{l}250 \\
190 \\
180 \\
123 \\
140 \\
120\end{array}$ & $\begin{array}{r}29 \\
31 \\
29 \\
27 \\
29 \\
- \\
-\end{array}$ \\
\hline MEAN & 74 & 70 & 34 & 16 & 19 & 518 & 324 & 115 & 117 & 63 & 224 & 45 \\
\hline WTR YR & 79 & EAN & 136 & $\max$ & 2930 & & & 8 & & & & \\
\hline TOTAL & 2294 & 2114 & 1083 & 510 & 557 & 16057 & 9711 & 3566 & 3508 & 1982 & 6942 & 1373 \\
\hline
\end{tabular}

TOTAL PHOSPHORUS, WATER YEAR OCTOBER 1979 TO SEPTEMBER 1980

\begin{tabular}{|c|c|c|c|c|c|c|c|c|c|c|c|c|}
\hline OAY & OCT & NOV & DEC & JAN & FER & MAR & APR & MAY & JUN & JUL. & AUG & 5EP \\
\hline $\begin{array}{l}1 \\
2 \\
3 \\
4 \\
5\end{array}$ & $\begin{array}{l}31 \\
31 \\
34 \\
31 \\
34\end{array}$ & $\begin{array}{l}49 \\
54 \\
49 \\
47 \\
41\end{array}$ & $\begin{array}{l}22 \\
19 \\
17 \\
17 \\
17\end{array}$ & $\begin{array}{l}33 \\
28 \\
26 \\
23 \\
22\end{array}$ & $\begin{array}{l}17 \\
18 \\
19 \\
19 \\
19\end{array}$ & $\begin{array}{l}17 \\
15 \\
13 \\
12 \\
14\end{array}$ & $\begin{array}{r}50 \\
40 \\
50 \\
295 \\
727\end{array}$ & $\begin{array}{r}110 \\
94 \\
81 \\
69 \\
62\end{array}$ & $\begin{array}{r}100 \\
92 \\
86 \\
82 \\
88\end{array}$ & $\begin{array}{l}38 \\
38 \\
38 \\
38 \\
35\end{array}$ & $\begin{array}{l}41 \\
38 \\
41 \\
44 \\
44\end{array}$ & $\begin{array}{r}110 \\
100 \\
96 \\
69 \\
64\end{array}$ \\
\hline $\begin{array}{r}6 \\
7 \\
8 \\
9 \\
10\end{array}$ & $\begin{array}{l}36 \\
36 \\
41 \\
47 \\
44\end{array}$ & $\begin{array}{l}44 \\
57 \\
57 \\
52 \\
47\end{array}$ & $\begin{array}{l}17 \\
19 \\
22 \\
40 \\
23\end{array}$ & $\begin{array}{l}20 \\
19 \\
17 \\
16 \\
15\end{array}$ & $\begin{array}{l}19 \\
18 \\
17 \\
15 \\
14\end{array}$ & $\begin{array}{l}14 \\
10 \\
10 \\
10 \\
10\end{array}$ & $\begin{array}{r}206 \\
162 \\
711 \\
1130 \\
659\end{array}$ & $\begin{array}{l}58 \\
53 \\
58 \\
49 \\
45\end{array}$ & $\begin{array}{l}492 \\
572 \\
649 \\
616 \\
591\end{array}$ & $\begin{array}{l}41 \\
41 \\
38 \\
32 \\
32\end{array}$ & $\begin{array}{r}49 \\
49 \\
612 \\
1160 \\
571\end{array}$ & $\begin{array}{l}54 \\
49 \\
46 \\
49 \\
56\end{array}$ \\
\hline $\begin{array}{l}11 \\
12 \\
13 \\
14 \\
15\end{array}$ & $\begin{array}{l}41 \\
39 \\
44 \\
36 \\
34\end{array}$ & $\begin{array}{l}44 \\
47 \\
44 \\
44 \\
44\end{array}$ & $\begin{array}{r}19 \\
16 \\
13 \\
12 \\
9\end{array}$ & $\begin{array}{r}13 \\
13 \\
12 \\
12 \\
100\end{array}$ & $\begin{array}{l}14 \\
14 \\
14 \\
13 \\
12\end{array}$ & $\begin{array}{r}10 \\
9 \\
9 \\
10 \\
11\end{array}$ & $\begin{array}{l}550 \\
340 \\
260 \\
210 \\
210\end{array}$ & $\begin{array}{r}47 \\
47 \\
28 \\
110 \\
140\end{array}$ & $\begin{array}{r}176 \\
77 \\
110 \\
110 \\
120\end{array}$ & $\begin{array}{l}32 \\
30 \\
30 \\
27 \\
32\end{array}$ & $\begin{array}{r}320 \\
233 \\
174 \\
117 \\
86\end{array}$ & $\begin{array}{l}52 \\
49 \\
77 \\
96 \\
91\end{array}$ \\
\hline $\begin{array}{l}16 \\
17 \\
18 \\
19 \\
20\end{array}$ & $\begin{array}{l}39 \\
39 \\
39 \\
41 \\
70\end{array}$ & $\begin{array}{l}52 \\
49 \\
47 \\
47 \\
47\end{array}$ & $\begin{array}{l}10 \\
10 \\
10 \\
10 \\
11\end{array}$ & $\begin{array}{l}350 \\
880 \\
600 \\
450 \\
230\end{array}$ & $\begin{array}{l}12 \\
11 \\
10 \\
11 \\
12\end{array}$ & $\begin{array}{r}14 \\
130 \\
170 \\
680 \\
490\end{array}$ & $\begin{array}{l}382 \\
310 \\
240 \\
200 \\
170\end{array}$ & $\begin{array}{l}130 \\
120 \\
130 \\
180 \\
170\end{array}$ & $\begin{array}{r}120 \\
89 \\
76 \\
82 \\
130\end{array}$ & $\begin{array}{r}59 \\
99 \\
59 \\
41 \\
663\end{array}$ & $\begin{array}{r}76 \\
71 \\
76 \\
81 \\
290\end{array}$ & $\begin{array}{r}99 \\
253 \\
174 \\
110 \\
213\end{array}$ \\
\hline $\begin{array}{l}21 \\
22 \\
23 \\
24 \\
25\end{array}$ & $\begin{array}{l}47 \\
44 \\
57 \\
79 \\
90\end{array}$ & $\begin{array}{l}49 \\
35 \\
50 \\
38 \\
27\end{array}$ & $\begin{array}{r}11 \\
12 \\
16 \\
23 \\
447\end{array}$ & $\begin{array}{r}120 \\
70 \\
40 \\
27 \\
24\end{array}$ & $\begin{array}{l}24 \\
32 \\
65 \\
45 \\
42\end{array}$ & $\begin{array}{r}230 \\
100 \\
60 \\
40 \\
40\end{array}$ & $\begin{array}{r}150 \\
130 \\
110 \\
98 \\
91\end{array}$ & $\begin{array}{r}120 \\
100 \\
88 \\
82 \\
76\end{array}$ & $\begin{array}{r}120 \\
89 \\
76 \\
61 \\
55\end{array}$ & $\begin{array}{r}867 \\
344 \\
170 \\
98 \\
73\end{array}$ & $\begin{array}{r}314 \\
236 \\
160 \\
99 \\
82\end{array}$ & $\begin{array}{r}545 \\
2650 \\
2800 \\
2240 \\
1170\end{array}$ \\
\hline $\begin{array}{l}26 \\
27 \\
28 \\
29 \\
30 \\
31\end{array}$ & $\begin{array}{l}70 \\
54 \\
54 \\
54 \\
47 \\
47\end{array}$ & $\begin{array}{r}38 \\
98 \\
93 \\
50 \\
25 \\
--\end{array}$ & $\begin{array}{r}353 \\
98 \\
61 \\
50 \\
40 \\
37\end{array}$ & $\begin{array}{l}23 \\
21 \\
19 \\
18 \\
17 \\
17\end{array}$ & $\begin{array}{r}44 \\
40 \\
30 \\
27 \\
--0 \\
---\end{array}$ & $\begin{array}{r}70 \\
98 \\
100 \\
80 \\
90 \\
60\end{array}$ & $\begin{array}{r}85 \\
79 \\
77 \\
83 \\
98 \\
- \\
-\end{array}$ & $\begin{array}{r}70 \\
64 \\
61 \\
67 \\
110 \\
110\end{array}$ & $\begin{array}{r}52 \\
46 \\
46 \\
52 \\
49 \\
- \\
-\end{array}$ & $\begin{array}{l}64 \\
67 \\
58 \\
49 \\
41 \\
38\end{array}$ & $\begin{array}{l}100 \\
120 \\
161 \\
190 \\
160 \\
120\end{array}$ & $\begin{array}{l}520 \\
320 \\
242 \\
183 \\
154 \\
---\end{array}$ \\
\hline MEAN & 46 & 48 & 47 & 106 & 22 & 84 & 263 & 88 & 170 & 107 & 191 & 424 \\
\hline WTR YP & 80 & & 33 & $\max$ & 2800 & \multicolumn{2}{|c|}{ MIN } & 9 & & & & \\
\hline TOTAL & 1430 & 1465 & 1481 & 3275 & 647 & 2626 & 7903 & 2729 & 5104 & 3312 & 5915 & 12731 \\
\hline
\end{tabular}

TOTAL LOAD FOR YEAR: 48618 POUNDS 
Table 29. Total orthophosphate phosphorus load, in pounds per day, for Onion River near Sheboygan Falls, 1979 and 1980 water years

TOIAL ORTHOPHOSPHORUS, WATER YEAR OCTOBER 1978 TO SEPTEMBER 1979

\begin{tabular}{|c|c|c|c|c|c|c|c|c|c|c|c|c|}
\hline DAY & OCT & Nov & DEC & JAN & FEB & MAR & APR & MAY & JUN & JUL. & AUG & SEF \\
\hline $\begin{array}{l}1 \\
2 \\
3 \\
4 \\
5\end{array}$ & $\begin{array}{r}110 \\
86 \\
61 \\
48 \\
38\end{array}$ & $\begin{array}{l}14 \\
15 \\
15 \\
15 \\
15\end{array}$ & $\begin{array}{l}32 \\
21 \\
21 \\
12 \\
12\end{array}$ & $\begin{array}{l}12 \\
12 \\
12 \\
11 \\
11\end{array}$ & $\begin{array}{l}14 \\
14 \\
14 \\
14 \\
14\end{array}$ & $\begin{array}{l}34 \\
35 \\
38 \\
46 \\
46\end{array}$ & $\begin{array}{l}854 \\
371 \\
190 \\
190 \\
210\end{array}$ & $\begin{array}{l}140 \\
110 \\
170 \\
100 \\
75\end{array}$ & $\begin{array}{r}9 \\
10 \\
8 \\
14 \\
10\end{array}$ & $\begin{array}{l}73 \\
53 \\
51 \\
34 \\
31\end{array}$ & $\begin{array}{l}23 \\
19 \\
17 \\
15 \\
15\end{array}$ & $\begin{array}{l}47 \\
44 \\
38 \\
30 \\
27\end{array}$ \\
\hline $\begin{array}{r}6 \\
7 \\
8 \\
9 \\
10\end{array}$ & $\begin{array}{r}120 \\
120 \\
95 \\
80 \\
66\end{array}$ & $\begin{array}{l}15 \\
15 \\
15 \\
15 \\
15\end{array}$ & $\begin{array}{l}\text { 11 } \\
\text { i1 } \\
\text { il } \\
\text { il } \\
\text { il }\end{array}$ & $\begin{array}{l}11 \\
11 \\
11 \\
11 \\
11\end{array}$ & $\begin{array}{l}14 \\
15 \\
15 \\
15 \\
16\end{array}$ & $\begin{array}{l}45 \\
45 \\
45 \\
45 \\
45\end{array}$ & $\begin{array}{r}150 \\
110 \\
69 \\
55 \\
54\end{array}$ & $\begin{array}{l}59 \\
45 \\
35 \\
29 \\
24\end{array}$ & $\begin{array}{r}9 \\
74 \\
59 \\
47 \\
130\end{array}$ & $\begin{array}{l}28 \\
22 \\
19 \\
17 \\
15\end{array}$ & $\begin{array}{r}25 \\
22 \\
17 \\
19 \\
330\end{array}$ & $\begin{array}{l}26 \\
23 \\
18 \\
16 \\
15\end{array}$ \\
\hline $\begin{array}{l}11 \\
12 \\
13 \\
14 \\
15\end{array}$ & $\begin{array}{l}56 \\
49 \\
40 \\
34 \\
30\end{array}$ & $\begin{array}{l}15 \\
15 \\
18 \\
23 \\
32\end{array}$ & $\begin{array}{r}11 \\
10 \\
10 \\
10 \\
9\end{array}$ & $\begin{array}{r}11 \\
11 \\
11 \\
8 \\
7\end{array}$ & $\begin{array}{l}16 \\
16 \\
16 \\
16 \\
16\end{array}$ & $\begin{array}{l}45 \\
47 \\
50 \\
51 \\
51\end{array}$ & $\begin{array}{r}59 \\
320 \\
410 \\
270 \\
140\end{array}$ & $\begin{array}{l}21 \\
20 \\
18 \\
17 \\
19\end{array}$ & $\begin{array}{l}87 \\
62 \\
50 \\
40 \\
36\end{array}$ & $\begin{array}{l}18 \\
22 \\
19 \\
26 \\
22\end{array}$ & $\begin{array}{r}425 \\
240 \\
107 \\
69 \\
49\end{array}$ & $\begin{array}{l}15 \\
16 \\
16 \\
15 \\
14\end{array}$ \\
\hline $\begin{array}{l}16 \\
17 \\
18 \\
19 \\
20\end{array}$ & $\begin{array}{l}32 \\
32 \\
31 \\
28 \\
30\end{array}$ & $\begin{array}{r}26 \\
51 \\
120 \\
110 \\
77\end{array}$ & $\begin{array}{l}9 \\
9 \\
9 \\
9 \\
9\end{array}$ & $\begin{array}{r}6 \\
7 \\
8 \\
9 \\
10\end{array}$ & $\begin{array}{l}15 \\
15 \\
16 \\
16 \\
16\end{array}$ & $\begin{array}{r}51 \\
51 \\
23 \\
180 \\
320\end{array}$ & $\begin{array}{l}74 \\
54 \\
41 \\
34 \\
29\end{array}$ & $\begin{array}{l}15 \\
17 \\
20 \\
39 \\
36\end{array}$ & $\begin{array}{l}34 \\
30 \\
28 \\
27 \\
62\end{array}$ & $\begin{array}{l}16 \\
13 \\
11 \\
12 \\
12\end{array}$ & $\begin{array}{l}35 \\
29 \\
27 \\
25 \\
46\end{array}$ & $\begin{array}{l}13 \\
13 \\
12 \\
11 \\
10\end{array}$ \\
\hline $\begin{array}{l}21 \\
22 \\
23 \\
24 \\
25\end{array}$ & $\begin{array}{l}28 \\
23 \\
16 \\
28 \\
33\end{array}$ & $\begin{array}{r}58 \\
45 \\
64 \\
120 \\
98\end{array}$ & $\begin{array}{l}8 \\
8 \\
8 \\
8 \\
B\end{array}$ & $\begin{array}{l}10 \\
10 \\
10 \\
10 \\
10\end{array}$ & $\begin{array}{l}22 \\
23 \\
25 \\
27 \\
29\end{array}$ & $\begin{array}{r}1070 \\
1120 \\
1330 \\
1320 \\
533\end{array}$ & $\begin{array}{l}26 \\
23 \\
21 \\
19 \\
24\end{array}$ & $\begin{array}{l}27 \\
21 \\
17 \\
15 \\
10\end{array}$ & $\begin{array}{l}50 \\
31 \\
24 \\
19 \\
17\end{array}$ & $\begin{array}{l}13 \\
14 \\
12 \\
11 \\
13\end{array}$ & $\begin{array}{r}167 \\
86 \\
100 \\
240 \\
188\end{array}$ & $\begin{array}{l}\text { I1 } \\
11 \\
11 \\
11 \\
\text { i1 }\end{array}$ \\
\hline $\begin{array}{l}26 \\
27 \\
28 \\
29 \\
30 \\
31\end{array}$ & $\begin{array}{l}32 \\
23 \\
18 \\
13 \\
13 \\
14\end{array}$ & $\begin{array}{r}77 \\
61 \\
53 \\
47 \\
41 \\
-\end{array}$ & $\begin{array}{l}8 \\
8 \\
8 \\
8 \\
8 \\
8\end{array}$ & $\begin{array}{l}10 \\
10 \\
10 \\
10 \\
10 \\
10\end{array}$ & $\begin{array}{r}30 \\
31 \\
33 \\
\ldots-. \\
\hdashline-\end{array}$ & $\begin{array}{r}320 \\
240 \\
200 \\
179 \\
570 \\
1360\end{array}$ & $\begin{array}{r}95 \\
120 \\
71 \\
44 \\
140 \\
-.-\end{array}$ & $\begin{array}{l}12 \\
11 \\
10 \\
10 \\
15 \\
17\end{array}$ & $\begin{array}{r}15 \\
14 \\
13 \\
47 \\
122 \\
-\end{array}$ & $\begin{array}{l}23 \\
21 \\
17 \\
15 \\
14 \\
20\end{array}$ & $\begin{array}{l}99 \\
76 \\
72 \\
45 \\
55 \\
46\end{array}$ & $\begin{array}{l}11 \\
12 \\
11 \\
10 \\
11 \\
-0\end{array}$ \\
\hline $\begin{array}{l}\operatorname{MAX} \\
\operatorname{MIN}\end{array}$ & $\begin{array}{r}120 \\
13\end{array}$ & $\begin{array}{r}120 \\
14\end{array}$ & $\begin{array}{r}32 \\
8.0\end{array}$ & $\begin{array}{r}12 \\
6.0\end{array}$ & $\begin{array}{l}33 \\
14\end{array}$ & $\begin{array}{r}1360 \\
23\end{array}$ & $\begin{array}{r}854 \\
19\end{array}$ & $\begin{array}{r}170 \\
10\end{array}$ & $\begin{array}{l}130 \\
8.0\end{array}$ & $\begin{array}{l}73 \\
11\end{array}$ & $\begin{array}{r}425 \\
15\end{array}$ & $\begin{array}{l}47 \\
10\end{array}$ \\
\hline
\end{tabular}

WTR YR 1979 MAX 1360 MIN 6.0

$\begin{array}{lllllllllllll}\text { TOTAL } & 1427 & 1300 & 336 & 311 & 523 & 9535 & 4267 & 1174 & 1178 & 687 & 2728 & 529\end{array}$ TOTAL LOAD FOR YEAR: 23995 POUNDS

TOTAL ORTHOPHOSPHORUS. WATER YEAR OCTOBER 1979 TO SEPTEMBER 1980

\begin{tabular}{|c|c|c|c|c|c|c|c|c|c|c|c|c|}
\hline DAY & OCr & NOV & DEC & JAN & FE8 & MAR & APR & MAY & JUN & JUL & AUG & SEP \\
\hline $\begin{array}{l}1 \\
2 \\
3 \\
4 \\
5\end{array}$ & $\begin{array}{l}11 \\
11 \\
12 \\
11 \\
12\end{array}$ & $\begin{array}{l}18 \\
20 \\
18 \\
17 \\
15\end{array}$ & $\begin{array}{l}20 \\
18 \\
17 \\
17 \\
17\end{array}$ & $\begin{array}{l}27 \\
24 \\
23 \\
21 \\
20\end{array}$ & $\begin{array}{l}17 \\
18 \\
19 \\
19 \\
19\end{array}$ & $\begin{array}{l}17 \\
15 \\
13 \\
12 \\
14\end{array}$ & $\begin{array}{r}48 \\
48 \\
52 \\
105 \\
228\end{array}$ & $\begin{array}{r}19 \\
16 \\
13 \\
10 \\
9\end{array}$ & $\begin{array}{l}20 \\
17 \\
15 \\
14 \\
14\end{array}$ & $\begin{array}{l}5 \\
5 \\
5 \\
5 \\
5\end{array}$ & $\begin{array}{r}10 \\
9 \\
10 \\
11 \\
11\end{array}$ & $\begin{array}{l}48 \\
45 \\
41 \\
27 \\
25\end{array}$ \\
\hline $\begin{array}{r}6 \\
7 \\
8 \\
9 \\
10\end{array}$ & $\begin{array}{l}13 \\
13 \\
15 \\
17 \\
16\end{array}$ & $\begin{array}{l}16 \\
54 \\
54 \\
45 \\
41\end{array}$ & $\begin{array}{l}17 \\
18 \\
20 \\
31 \\
21\end{array}$ & $\begin{array}{l}19 \\
18 \\
17 \\
16 \\
15\end{array}$ & $\begin{array}{l}19 \\
18 \\
17 \\
15 \\
14\end{array}$ & $\begin{array}{l}14 \\
10 \\
10 \\
10 \\
10\end{array}$ & $\begin{array}{r}105 \\
80 \\
206 \\
341 \\
243\end{array}$ & $\begin{array}{l}8 \\
7 \\
4 \\
6 \\
6\end{array}$ & $\begin{array}{l}176 \\
210 \\
217 \\
209 \\
177\end{array}$ & $\begin{array}{l}6 \\
6 \\
5 \\
4 \\
4\end{array}$ & $\begin{array}{r}13 \\
11 \\
344 \\
731 \\
350\end{array}$ & $\begin{array}{l}20 \\
18 \\
17 \\
18 \\
21\end{array}$ \\
\hline $\begin{array}{l}11 \\
12 \\
13 \\
14 \\
15\end{array}$ & $\begin{array}{l}15 \\
14 \\
16 \\
13 \\
12\end{array}$ & $\begin{array}{l}39 \\
41 \\
39 \\
39 \\
39\end{array}$ & $\begin{array}{r}18 \\
16 \\
14 \\
8 \\
12\end{array}$ & $\begin{array}{l}14 \\
14 \\
13 \\
13 \\
24\end{array}$ & $\begin{array}{l}14 \\
14 \\
14 \\
13 \\
12\end{array}$ & $\begin{array}{r}10 \\
9 \\
9 \\
10 \\
11\end{array}$ & $\begin{array}{r}203 \\
100 \\
69 \\
50 \\
51\end{array}$ & $\begin{array}{r}6 \\
6 \\
6 \\
21 \\
28\end{array}$ & $\begin{array}{l}59 \\
30 \\
22 \\
21 \\
25\end{array}$ & $\begin{array}{l}4 \\
4 \\
4 \\
3 \\
4\end{array}$ & $\begin{array}{r}179 \\
123 \\
88 \\
56 \\
39\end{array}$ & $\begin{array}{l}19 \\
18 \\
22 \\
28 \\
26\end{array}$ \\
\hline $\begin{array}{l}16 \\
17 \\
18 \\
19 \\
20\end{array}$ & $\begin{array}{l}14 \\
14 \\
14 \\
15 \\
26\end{array}$ & $\begin{array}{l}41 \\
43 \\
41 \\
41 \\
41\end{array}$ & $\begin{array}{l}11 \\
11 \\
11 \\
11 \\
12\end{array}$ & $\begin{array}{r}75 \\
390 \\
380 \\
380 \\
220\end{array}$ & $\begin{array}{l}12 \\
11 \\
10 \\
11 \\
12\end{array}$ & $\begin{array}{r}27 \\
44 \\
124 \\
466 \\
370\end{array}$ & $\begin{array}{r}120 \\
89 \\
62 \\
47 \\
37\end{array}$ & $\begin{array}{l}27 \\
23 \\
26 \\
39 \\
36\end{array}$ & $\begin{array}{l}24 \\
16 \\
13 \\
14 \\
27\end{array}$ & $\begin{array}{r}14 \\
24 \\
15 \\
10 \\
261\end{array}$ & $\begin{array}{r}34 \\
29 \\
30 \\
30 \\
110\end{array}$ & $\begin{array}{r}22 \\
116 \\
63 \\
41 \\
111\end{array}$ \\
\hline $\begin{array}{l}21 \\
22 \\
23 \\
24 \\
25\end{array}$ & $\begin{array}{l}17 \\
16 \\
21 \\
29 \\
33\end{array}$ & $\begin{array}{l}43 \\
28 \\
37 \\
30 \\
24\end{array}$ & $\begin{array}{r}12 \\
13 \\
16 \\
21 \\
190\end{array}$ & $\begin{array}{r}130 \\
70 \\
45 \\
22 \\
19\end{array}$ & $\begin{array}{l}24 \\
32 \\
65 \\
61 \\
42\end{array}$ & $\begin{array}{r}155 \\
100 \\
66 \\
40 \\
42\end{array}$ & $\begin{array}{l}30 \\
25 \\
21 \\
17 \\
15\end{array}$ & $\begin{array}{l}25 \\
19 \\
16 \\
14 \\
13\end{array}$ & $\begin{array}{r}25 \\
16 \\
13 \\
10 \\
8\end{array}$ & $\begin{array}{r}315 \\
120 \\
62 \\
31 \\
21\end{array}$ & $\begin{array}{r}230 \\
140 \\
72 \\
42 \\
34\end{array}$ & $\begin{array}{r}344 \\
924 \\
1570 \\
1390 \\
735\end{array}$ \\
\hline $\begin{array}{l}26 \\
27 \\
28 \\
29 \\
30 \\
31\end{array}$ & $\begin{array}{l}26 \\
20 \\
20 \\
20 \\
17 \\
17\end{array}$ & $\begin{array}{r}30 \\
61 \\
59 \\
37 \\
22 \\
-\end{array}$ & $\begin{array}{r}160 \\
61 \\
43 \\
37 \\
31 \\
29\end{array}$ & $\begin{array}{l}18 \\
16 \\
16 \\
14 \\
13 \\
13\end{array}$ & $\begin{array}{r}44 \\
40 \\
30 \\
27 \\
- \\
\end{array}$ & $\begin{array}{l}54 \\
71 \\
95 \\
75 \\
80 \\
58\end{array}$ & $\begin{array}{r}14 \\
12 \\
12 \\
13 \\
17 \\
- \\
\end{array}$ & $\begin{array}{l}12 \\
10 \\
10 \\
11 \\
21 \\
22\end{array}$ & $\begin{array}{r}8 \\
7 \\
7 \\
8 \\
7 \\
-\end{array}$ & $\begin{array}{l}18 \\
19 \\
16 \\
13 \\
10 \\
10\end{array}$ & $\begin{array}{l}45 \\
51 \\
51 \\
90 \\
74 \\
53\end{array}$ & $\begin{array}{r}327 \\
186 \\
129 \\
93 \\
78 \\
-\end{array}$ \\
\hline $1 \mathrm{~A}$ & $\begin{array}{l}33 \\
11\end{array}$ & $\begin{array}{l}61 \\
15\end{array}$ & $\begin{array}{l}190 \\
8.0\end{array}$ & $\begin{array}{r}390 \\
13\end{array}$ & $\begin{array}{l}65 \\
10\end{array}$ & $\begin{array}{l}466 \\
9.0\end{array}$ & $\begin{array}{r}341 \\
12\end{array}$ & $\begin{array}{r}39 \\
4.0\end{array}$ & $\begin{array}{l}217 \\
7.0\end{array}$ & $\begin{array}{l}315 \\
3.0\end{array}$ & $\begin{array}{l}731 \\
9.0\end{array}$ & $\begin{array}{r}1570 \\
17\end{array}$ \\
\hline
\end{tabular}

WTR YR 1980 MAX 1570 MIN 3.0

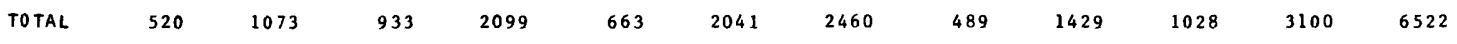
TOTAL LOAD FOR YEAR: 22357 POUNDS 
Table 30. Specific conductance for Onion River at Hingham, 1979 and 1980 water years

SPECIFIC CONDUCTANCE (MICROMHOS/CM AT 25 DEG. C), WATER YEAR OCTOBER 1978 TO SEPTEMBER 1979

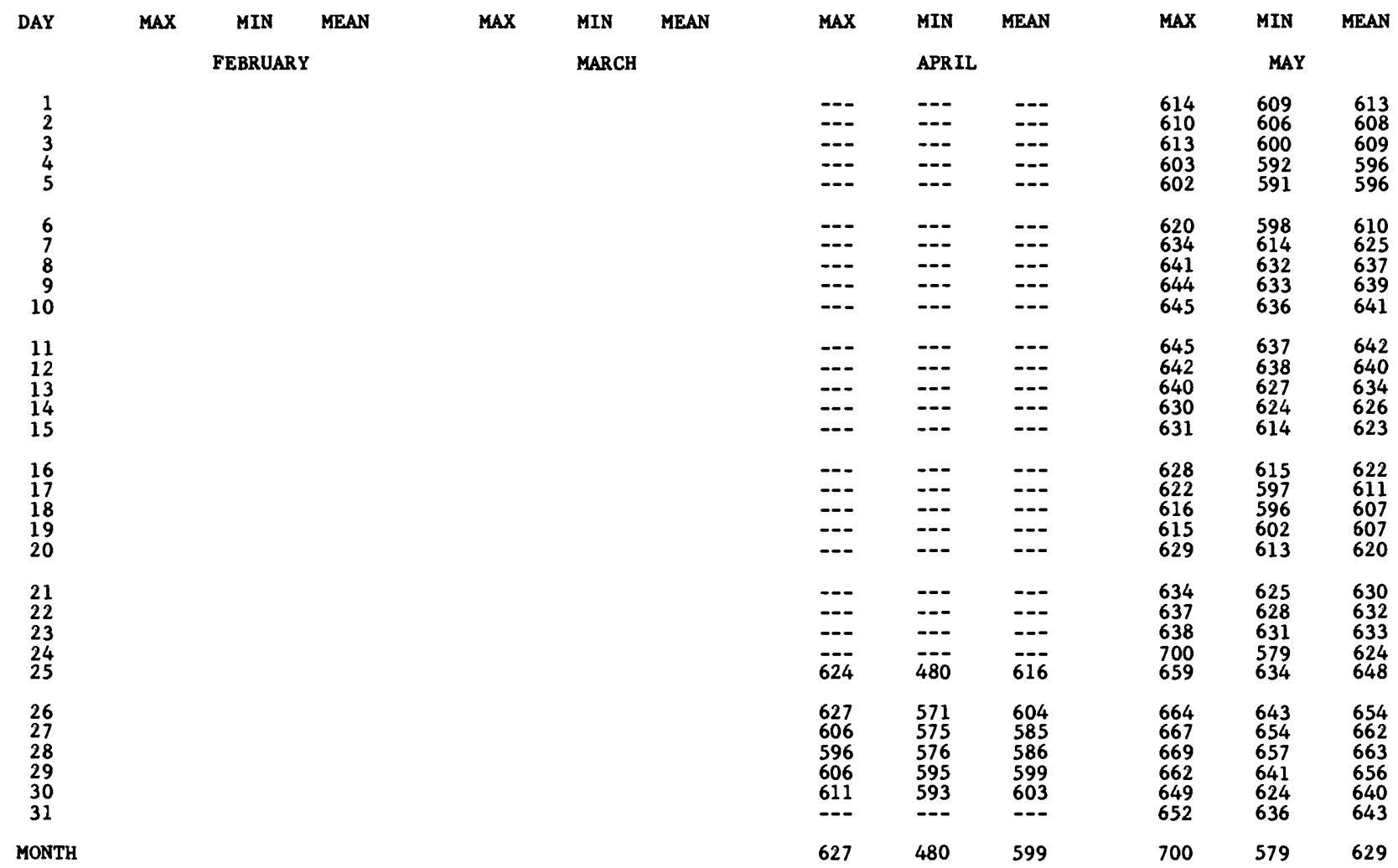

SPECIFIC CONDUCTANCE (MICROMHOS/CM AT 25 DEG. C), WATER YEAR OCTOBER 1978 TO SEPTEMBER 1979

\begin{tabular}{|c|c|c|c|c|c|c|c|c|c|c|c|c|}
\hline \multirow[t]{2}{*}{ DAY } & $\max$ & MIN & MEAN & MAX & MIN & MEAN & MAX & MIN & MEAN & $\operatorname{MAX}$ & MIN & MEAN \\
\hline & \multicolumn{3}{|c|}{ JUNE } & \multicolumn{3}{|c|}{ JULY } & \multicolumn{3}{|c|}{ AUGUST } & \multicolumn{3}{|c|}{ SEPTEMBER } \\
\hline $\begin{array}{l}1 \\
2 \\
3 \\
4 \\
5\end{array}$ & $\begin{array}{l}648 \\
647 \\
646 \\
646 \\
648\end{array}$ & $\begin{array}{l}638 \\
635 \\
628 \\
636 \\
639\end{array}$ & $\begin{array}{l}643 \\
640 \\
636 \\
643 \\
642\end{array}$ & $\begin{array}{l}492 \\
522 \\
524 \\
522 \\
538\end{array}$ & $\begin{array}{l}477 \\
486 \\
513 \\
513 \\
526\end{array}$ & $\begin{array}{l}486 \\
507 \\
520 \\
518 \\
531\end{array}$ & $\begin{array}{l}584 \\
580 \\
567 \\
509 \\
510\end{array}$ & $\begin{array}{l}563 \\
565 \\
478 \\
496 \\
474\end{array}$ & $\begin{array}{l}578 \\
575 \\
531 \\
504 \\
501\end{array}$ & $\begin{array}{l}600 \\
594 \\
589 \\
591 \\
588\end{array}$ & $\begin{array}{l}584 \\
576 \\
578 \\
575 \\
567\end{array}$ & $\begin{array}{l}592 \\
587 \\
583 \\
586 \\
577\end{array}$ \\
\hline $\begin{array}{r}6 \\
7 \\
8 \\
9 \\
10\end{array}$ & $\begin{array}{l}647 \\
637 \\
624 \\
609 \\
606\end{array}$ & $\begin{array}{l}632 \\
616 \\
583 \\
591 \\
553\end{array}$ & $\begin{array}{l}641 \\
629 \\
603 \\
603 \\
596\end{array}$ & $\begin{array}{l}542 \\
528 \\
488 \\
493 \\
497\end{array}$ & $\begin{array}{l}528 \\
489 \\
475 \\
473 \\
473\end{array}$ & $\begin{array}{l}536 \\
510 \\
484 \\
482 \\
489\end{array}$ & $\begin{array}{l}507 \\
507 \\
648 \\
535 \\
489\end{array}$ & $\begin{array}{l}499 \\
498 \\
468 \\
404 \\
249\end{array}$ & $\begin{array}{l}502 \\
501 \\
509 \\
507 \\
374\end{array}$ & $\begin{array}{l}575 \\
566 \\
567 \\
561 \\
553\end{array}$ & $\begin{array}{l}555 \\
558 \\
554 \\
550 \\
544\end{array}$ & $\begin{array}{l}564 \\
562 \\
561 \\
557 \\
549\end{array}$ \\
\hline $\begin{array}{l}11 \\
12 \\
13 \\
14 \\
15\end{array}$ & $\begin{array}{l}613 \\
610 \\
601 \\
611 \\
610\end{array}$ & $\begin{array}{l}589 \\
594 \\
593 \\
598 \\
600\end{array}$ & $\begin{array}{l}604 \\
602 \\
598 \\
602 \\
606\end{array}$ & $\begin{array}{l}509 \\
492 \\
511 \\
535 \\
541\end{array}$ & $\begin{array}{l}479 \\
481 \\
485 \\
511 \\
532\end{array}$ & $\begin{array}{l}491 \\
487 \\
496 \\
523 \\
537\end{array}$ & $\begin{array}{l}419 \\
469 \\
521 \\
574 \\
597\end{array}$ & $\begin{array}{l}385 \\
422 \\
467 \\
506 \\
439\end{array}$ & $\begin{array}{l}395 \\
448 \\
496 \\
542 \\
594\end{array}$ & $\begin{array}{l}565 \\
580 \\
623 \\
591 \\
595\end{array}$ & $\begin{array}{l}549 \\
565 \\
539 \\
580 \\
583\end{array}$ & $\begin{array}{l}560 \\
571 \\
580 \\
587 \\
589\end{array}$ \\
\hline $\begin{array}{l}16 \\
17 \\
18 \\
19 \\
20\end{array}$ & $\begin{array}{l}625 \\
649 \\
590 \\
581 \\
567\end{array}$ & $\begin{array}{l}594 \\
585 \\
579 \\
560 \\
548\end{array}$ & $\begin{array}{l}607 \\
605 \\
586 \\
570 \\
559\end{array}$ & $\begin{array}{l}549 \\
556 \\
555 \\
553 \\
546\end{array}$ & $\begin{array}{l}536 \\
545 \\
503 \\
494 \\
525\end{array}$ & $\begin{array}{l}543 \\
550 \\
538 \\
535 \\
533\end{array}$ & $\begin{array}{l}609 \\
616 \\
630 \\
635 \\
623\end{array}$ & $\begin{array}{l}581 \\
588 \\
605 \\
625 \\
543\end{array}$ & $\begin{array}{l}596 \\
600 \\
618 \\
629 \\
601\end{array}$ & $\begin{array}{l}595 \\
599 \\
597 \\
585 \\
586\end{array}$ & $\begin{array}{l}589 \\
587 \\
585 \\
577 \\
575\end{array}$ & $\begin{array}{l}592 \\
595 \\
590 \\
582 \\
581\end{array}$ \\
\hline $\begin{array}{l}21 \\
22 \\
23 \\
24 \\
25\end{array}$ & $\begin{array}{l}549 \\
542 \\
537 \\
543 \\
563\end{array}$ & $\begin{array}{l}536 \\
522 \\
522 \\
534 \\
538\end{array}$ & $\begin{array}{l}544 \\
531 \\
531 \\
539 \\
551\end{array}$ & $\begin{array}{l}537 \\
522 \\
524 \\
530 \\
521\end{array}$ & $\begin{array}{l}507 \\
515 \\
517 \\
506 \\
508\end{array}$ & $\begin{array}{l}522 \\
520 \\
520 \\
523 \\
515\end{array}$ & $\begin{array}{l}584 \\
572 \\
547 \\
556 \\
592\end{array}$ & $\begin{array}{l}536 \\
454 \\
500 \\
524 \\
562\end{array}$ & $\begin{array}{l}566 \\
549 \\
533 \\
541 \\
579\end{array}$ & $\begin{array}{l}584 \\
576 \\
569 \\
554 \\
530\end{array}$ & $\begin{array}{l}570 \\
555 \\
553 \\
525 \\
499\end{array}$ & $\begin{array}{l}576 \\
570 \\
561 \\
538 \\
516\end{array}$ \\
\hline $\begin{array}{l}26 \\
27 \\
28 \\
29 \\
30 \\
31\end{array}$ & $\begin{array}{l}573 \\
594 \\
584 \\
569 \\
495 \\
---\end{array}$ & $\begin{array}{l}555 \\
548 \\
559 \\
387 \\
471 \\
---\end{array}$ & $\begin{array}{l}563 \\
567 \\
575 \\
509 \\
483 \\
--\end{array}$ & $\begin{array}{l}532 \\
551 \\
559 \\
577 \\
581 \\
574\end{array}$ & $\begin{array}{l}512 \\
529 \\
546 \\
557 \\
537 \\
559\end{array}$ & $\begin{array}{l}522 \\
544 \\
554 \\
567 \\
567 \\
564\end{array}$ & $\begin{array}{l}593 \\
591 \\
595 \\
594 \\
599 \\
595\end{array}$ & $\begin{array}{l}590 \\
585 \\
586 \\
587 \\
569 \\
579\end{array}$ & $\begin{array}{l}592 \\
589 \\
590 \\
591 \\
582 \\
587\end{array}$ & $\begin{array}{l}502 \\
492 \\
482 \\
478 \\
481 \\
---\end{array}$ & $\begin{array}{l}476 \\
471 \\
469 \\
468 \\
471 \\
---\end{array}$ & $\begin{array}{l}492 \\
482 \\
477 \\
473 \\
475 \\
---\end{array}$ \\
\hline MONTH & 649 & 387 & 587 & 581 & 473 & 523 & 648 & 249 & 545 & 623 & 468 & 557 \\
\hline
\end{tabular}

YEAR $\quad 700 \quad 249 \quad 569$

NOTE: NUMBER OF MISSING DAYS OF RECORD EXCEEDED $20 \%$ OF YEAR 
Table 30. Specific conductance for Onion River at Hingham, 1979 and 1980 water years-Continued

SPECIFIC CONDUCTANCE (MICROMHOS/CM AT 25 DEG. C), WATER YEAR OCTOBER 1979 TO SEPTEMBER 1980

\begin{tabular}{|c|c|c|c|c|c|c|c|c|c|c|c|c|}
\hline \multirow[t]{2}{*}{ DAY } & MAX & MIN & MEAN & MAX & MIN & MEAN & $\operatorname{MAX}$ & MIN & MEAN & MAX & MIN & MEAN \\
\hline & \multicolumn{3}{|c|}{ OCTOBER } & \multicolumn{3}{|c|}{ NOVEMBER } & \multicolumn{3}{|c|}{ DECEMBER } & \multicolumn{3}{|c|}{ JANUARY } \\
\hline $\begin{array}{l}1 \\
2 \\
3 \\
4 \\
5\end{array}$ & $\begin{array}{l}484 \\
493 \\
493 \\
500 \\
510\end{array}$ & $\begin{array}{l}469 \\
480 \\
489 \\
490 \\
497\end{array}$ & $\begin{array}{l}476 \\
487 \\
491 \\
494 \\
503\end{array}$ & $\begin{array}{l}575 \\
562 \\
563 \\
563 \\
569\end{array}$ & $\begin{array}{l}562 \\
552 \\
550 \\
558 \\
561\end{array}$ & $\begin{array}{l}568 \\
559 \\
557 \\
561 \\
565\end{array}$ & $\begin{array}{l}681 \\
700 \\
711 \\
715 \\
704\end{array}$ & $\begin{array}{l}651 \\
677 \\
700 \\
696 \\
670\end{array}$ & $\begin{array}{l}665 \\
692 \\
705 \\
710 \\
686\end{array}$ & $\begin{array}{l}652 \\
653 \\
654 \\
657 \\
661\end{array}$ & $\begin{array}{l}646 \\
643 \\
647 \\
651 \\
652\end{array}$ & $\begin{array}{l}651 \\
648 \\
650 \\
655 \\
657\end{array}$ \\
\hline $\begin{array}{r}6 \\
7 \\
8 \\
9 \\
10\end{array}$ & $\begin{array}{l}514 \\
517 \\
520 \\
522 \\
527\end{array}$ & $\begin{array}{l}508 \\
511 \\
509 \\
520 \\
521\end{array}$ & $\begin{array}{l}511 \\
514 \\
515 \\
521 \\
523\end{array}$ & $\begin{array}{l}567 \\
559 \\
555 \\
558 \\
558\end{array}$ & $\begin{array}{l}554 \\
554 \\
548 \\
553 \\
553\end{array}$ & $\begin{array}{l}558 \\
555 \\
554 \\
554 \\
554\end{array}$ & $\begin{array}{l}677 \\
651 \\
636 \\
633 \\
633\end{array}$ & $\begin{array}{l}645 \\
633 \\
620 \\
617 \\
620\end{array}$ & $\begin{array}{l}657 \\
640 \\
627 \\
625 \\
628\end{array}$ & $\begin{array}{l}662 \\
684 \\
677 \\
712 \\
717\end{array}$ & $\begin{array}{l}653 \\
663 \\
661 \\
661 \\
694\end{array}$ & $\begin{array}{l}659 \\
674 \\
668 \\
687 \\
702\end{array}$ \\
\hline $\begin{array}{l}11 \\
12 \\
13 \\
14 \\
15\end{array}$ & $\begin{array}{l}521 \\
526 \\
520 \\
527 \\
532\end{array}$ & $\begin{array}{l}517 \\
509 \\
514 \\
518 \\
519\end{array}$ & $\begin{array}{l}519 \\
518 \\
518 \\
523 \\
525\end{array}$ & $\begin{array}{l}569 \\
583 \\
586 \\
573 \\
642\end{array}$ & $\begin{array}{l}552 \\
570 \\
568 \\
565 \\
563\end{array}$ & $\begin{array}{l}560 \\
575 \\
578 \\
568 \\
610\end{array}$ & $\begin{array}{l}623 \\
623 \\
626 \\
622 \\
632\end{array}$ & $\begin{array}{l}614 \\
606 \\
590 \\
600 \\
610\end{array}$ & $\begin{array}{l}618 \\
615 \\
608 \\
613 \\
618\end{array}$ & $\begin{array}{l}718 \\
673 \\
677 \\
685 \\
632\end{array}$ & $\begin{array}{l}660 \\
651 \\
655 \\
625 \\
620\end{array}$ & $\begin{array}{l}686 \\
661 \\
669 \\
648 \\
625\end{array}$ \\
\hline $\begin{array}{l}16 \\
17 \\
18 \\
19 \\
20\end{array}$ & $\begin{array}{l}530 \\
529 \\
536 \\
543 \\
574\end{array}$ & $\begin{array}{l}524 \\
524 \\
527 \\
530 \\
537\end{array}$ & $\begin{array}{l}527 \\
528 \\
532 \\
538 \\
549\end{array}$ & $\begin{array}{l}639 \\
627 \\
608 \\
614 \\
611\end{array}$ & $\begin{array}{l}620 \\
595 \\
592 \\
600 \\
600\end{array}$ & $\begin{array}{l}628 \\
605 \\
600 \\
606 \\
606\end{array}$ & $\begin{array}{l}645 \\
655 \\
691 \\
707 \\
673\end{array}$ & $\begin{array}{l}633 \\
624 \\
653 \\
672 \\
652\end{array}$ & $\begin{array}{l}637 \\
644 \\
666 \\
687 \\
659\end{array}$ & $\begin{array}{l}624 \\
458 \\
422 \\
485 \\
546\end{array}$ & $\begin{array}{l}448 \\
385 \\
387 \\
425 \\
479\end{array}$ & $\begin{array}{l}542 \\
420 \\
400 \\
454 \\
511\end{array}$ \\
\hline $\begin{array}{l}21 \\
22 \\
23 \\
24 \\
25\end{array}$ & $\begin{array}{l}556 \\
551 \\
544 \\
538 \\
543\end{array}$ & $\begin{array}{l}551 \\
542 \\
529 \\
528 \\
539\end{array}$ & $\begin{array}{l}555 \\
548 \\
532 \\
533 \\
541\end{array}$ & $\begin{array}{l}608 \\
614 \\
620 \\
627 \\
639\end{array}$ & $\begin{array}{l}590 \\
595 \\
598 \\
614 \\
620\end{array}$ & $\begin{array}{l}600 \\
602 \\
613 \\
619 \\
627\end{array}$ & $\begin{array}{l}659 \\
647 \\
614 \\
586 \\
549\end{array}$ & $\begin{array}{l}646 \\
613 \\
580 \\
537 \\
521\end{array}$ & $\begin{array}{l}651 \\
631 \\
597 \\
566 \\
540\end{array}$ & $\begin{array}{l}600 \\
628 \\
648 \\
660 \\
612\end{array}$ & $\begin{array}{l}545 \\
598 \\
610 \\
588 \\
578\end{array}$ & $\begin{array}{l}576 \\
619 \\
633 \\
625 \\
600\end{array}$ \\
\hline $\begin{array}{l}26 \\
27 \\
28 \\
29 \\
30 \\
31\end{array}$ & $\begin{array}{l}544 \\
551 \\
560 \\
581 \\
582 \\
582\end{array}$ & $\begin{array}{l}533 \\
542 \\
545 \\
555 \\
574 \\
553\end{array}$ & $\begin{array}{l}539 \\
547 \\
554 \\
572 \\
578 \\
574\end{array}$ & $\begin{array}{l}633 \\
639 \\
630 \\
623 \\
654 \\
---\end{array}$ & $\begin{array}{l}611 \\
627 \\
603 \\
603 \\
623 \\
---\end{array}$ & $\begin{array}{l}626 \\
633 \\
615 \\
610 \\
644 \\
---\end{array}$ & $\begin{array}{l}535 \\
575 \\
608 \\
631 \\
641 \\
651\end{array}$ & $\begin{array}{l}513 \\
533 \\
573 \\
609 \\
629 \\
642\end{array}$ & $\begin{array}{l}523 \\
552 \\
592 \\
623 \\
636 \\
646\end{array}$ & $\begin{array}{l}600 \\
633 \\
629 \\
636 \\
646 \\
649\end{array}$ & $\begin{array}{l}563 \\
591 \\
617 \\
609 \\
627 \\
638\end{array}$ & $\begin{array}{l}581 \\
611 \\
622 \\
622 \\
636 \\
646\end{array}$ \\
\hline MONTH & 582 & 469 & 529 & 654 & 548 & 590 & 715 & 513 & 631 & 718 & 385 & 614 \\
\hline
\end{tabular}

SPECIFIC CONDUCTANCE (MICROMHOS/CM AT 25 DEg. C), WATER YEAR OCTOBER 1979 TO SEPTEMBER 1980

\begin{tabular}{|c|c|c|c|c|c|c|c|c|c|c|c|c|}
\hline \multirow[t]{2}{*}{ DAY } & $\operatorname{MAX}$ & MIN & MEAN & $\operatorname{MAX}$ & MIN & MEAN & MAX & MIN & MEAN & $\operatorname{MAX}$ & MIN & MEAN \\
\hline & \multicolumn{3}{|c|}{ FEBRUARY } & \multicolumn{3}{|c|}{ MARCH } & \multicolumn{3}{|c|}{ APRIL } & \multicolumn{3}{|c|}{ MAY } \\
\hline $\begin{array}{l}1 \\
2 \\
3 \\
4 \\
5\end{array}$ & $\begin{array}{l}645 \\
648 \\
643 \\
615 \\
634\end{array}$ & $\begin{array}{l}629 \\
625 \\
608 \\
593 \\
603\end{array}$ & $\begin{array}{l}635 \\
633 \\
624 \\
606 \\
613\end{array}$ & $\begin{array}{l}670 \\
670 \\
662 \\
635 \\
619\end{array}$ & $\begin{array}{l}625 \\
616 \\
619 \\
604 \\
515\end{array}$ & $\begin{array}{l}642 \\
647 \\
643 \\
621 \\
579\end{array}$ & $\begin{array}{l}570 \\
569 \\
566 \\
571 \\
576\end{array}$ & $\begin{array}{l}542 \\
537 \\
523 \\
545 \\
548\end{array}$ & $\begin{array}{l}559 \\
554 \\
546 \\
561 \\
565\end{array}$ & $\begin{array}{l}690 \\
698 \\
705 \\
706 \\
710\end{array}$ & $\begin{array}{l}683 \\
678 \\
690 \\
694 \\
692\end{array}$ & $\begin{array}{l}686 \\
688 \\
695 \\
701 \\
701\end{array}$ \\
\hline $\begin{array}{r}6 \\
7 \\
8 \\
9 \\
10\end{array}$ & $\begin{array}{l}625 \\
605 \\
596 \\
577 \\
580\end{array}$ & $\begin{array}{l}602 \\
586 \\
571 \\
552 \\
561\end{array}$ & $\begin{array}{l}614 \\
596 \\
582 \\
566 \\
569\end{array}$ & $\begin{array}{l}607 \\
641 \\
628 \\
625 \\
619\end{array}$ & $\begin{array}{l}510 \\
569 \\
478 \\
478 \\
504\end{array}$ & $\begin{array}{l}566 \\
590 \\
562 \\
558 \\
567\end{array}$ & $\begin{array}{l}580 \\
596 \\
608 \\
572 \\
593\end{array}$ & $\begin{array}{l}549 \\
566 \\
489 \\
543 \\
549\end{array}$ & $\begin{array}{l}566 \\
582 \\
563 \\
555 \\
569\end{array}$ & $\begin{array}{l}702 \\
705 \\
689 \\
684 \\
645\end{array}$ & $\begin{array}{l}691 \\
666 \\
667 \\
630 \\
613\end{array}$ & $\begin{array}{l}698 \\
688 \\
677 \\
657 \\
631\end{array}$ \\
\hline $\begin{array}{l}11 \\
12 \\
13 \\
14 \\
15\end{array}$ & $\begin{array}{l}589 \\
589 \\
--- \\
---\end{array}$ & $\begin{array}{l}577 \\
555 \\
--- \\
---\end{array}$ & $\begin{array}{c}584 \\
581 \\
--- \\
---\end{array}$ & $\begin{array}{l}597 \\
613 \\
628 \\
659 \\
625\end{array}$ & $\begin{array}{l}510 \\
559 \\
572 \\
604 \\
597\end{array}$ & $\begin{array}{l}555 \\
582 \\
593 \\
618 \\
613\end{array}$ & $\begin{array}{l}618 \\
646 \\
657 \\
664 \\
662\end{array}$ & $\begin{array}{l}596 \\
622 \\
644 \\
651 \\
646\end{array}$ & $\begin{array}{l}607 \\
633 \\
652 \\
659 \\
655\end{array}$ & $\begin{array}{l}631 \\
598 \\
585 \\
602 \\
624\end{array}$ & $\begin{array}{l}579 \\
570 \\
559 \\
576 \\
594\end{array}$ & $\begin{array}{l}608 \\
586 \\
573 \\
586 \\
611\end{array}$ \\
\hline $\begin{array}{l}16 \\
17 \\
18 \\
19 \\
20\end{array}$ & $\begin{array}{l}--- \\
=-- \\
--- \\
---\end{array}$ & $\begin{array}{l}--- \\
-:- \\
--- \\
---\end{array}$ & $\begin{array}{l}--- \\
=-- \\
--- \\
---\end{array}$ & $\begin{array}{l}607 \\
469 \\
334 \\
373 \\
385\end{array}$ & $\begin{array}{l}419 \\
297 \\
301 \\
327 \\
361\end{array}$ & $\begin{array}{l}559 \\
370 \\
321 \\
357 \\
372\end{array}$ & $\begin{array}{l}666 \\
670 \\
682 \\
693 \\
697\end{array}$ & $\begin{array}{l}655 \\
662 \\
663 \\
677 \\
688\end{array}$ & $\begin{array}{l}660 \\
666 \\
671 \\
686 \\
691\end{array}$ & $\begin{array}{l}644 \\
646 \\
642 \\
660 \\
666\end{array}$ & $\begin{array}{l}616 \\
627 \\
631 \\
641 \\
651\end{array}$ & $\begin{array}{l}630 \\
638 \\
638 \\
6550 \\
658\end{array}$ \\
\hline $\begin{array}{l}21 \\
22 \\
23 \\
24 \\
25\end{array}$ & $\begin{array}{l}--- \\
=-- \\
--- \\
---\end{array}$ & $\begin{array}{l}--- \\
--- \\
--- \\
---\end{array}$ & $\begin{array}{l}--- \\
=-- \\
--- \\
---\end{array}$ & $\begin{array}{l}404 \\
445 \\
482 \\
515 \\
528\end{array}$ & $\begin{array}{l}363 \\
395 \\
439 \\
480 \\
474\end{array}$ & $\begin{array}{l}390 \\
420 \\
457 \\
491 \\
503\end{array}$ & $\begin{array}{l}698 \\
705 \\
706 \\
698 \\
696\end{array}$ & $\begin{array}{l}686 \\
693 \\
694 \\
692 \\
690\end{array}$ & $\begin{array}{l}692 \\
698 \\
699 \\
693 \\
692\end{array}$ & $\begin{array}{l}671 \\
670 \\
661 \\
654 \\
643\end{array}$ & $\begin{array}{l}655 \\
656 \\
647 \\
638 \\
625\end{array}$ & $\begin{array}{l}659 \\
659 \\
652 \\
646 \\
636\end{array}$ \\
\hline $\begin{array}{l}26 \\
27 \\
28 \\
29 \\
30 \\
31\end{array}$ & $\begin{array}{l}--- \\
594 \\
622 \\
635 \\
--- \\
---\end{array}$ & $\begin{array}{l}57 \\
572 \\
572 \\
588 \\
---\end{array}$ & $\begin{array}{l}--- \\
585 \\
598 \\
615 \\
--- \\
--\end{array}$ & $\begin{array}{l}625 \\
620 \\
581 \\
562 \\
579 \\
573\end{array}$ & $\begin{array}{l}474 \\
580 \\
530 \\
534 \\
555 \\
541\end{array}$ & $\begin{array}{l}611 \\
594 \\
552 \\
549 \\
570 \\
564\end{array}$ & $\begin{array}{l}697 \\
698 \\
690 \\
688 \\
689 \\
---\end{array}$ & $\begin{array}{l}691 \\
689 \\
687 \\
683 \\
681 \\
--\end{array}$ & $\begin{array}{l}693 \\
694 \\
688 \\
684 \\
683 \\
---\end{array}$ & $\begin{array}{l}627 \\
620 \\
606 \\
595 \\
586 \\
575\end{array}$ & $\begin{array}{l}608 \\
601 \\
587 \\
580 \\
564 \\
552\end{array}$ & $\begin{array}{l}620 \\
607 \\
597 \\
588 \\
575 \\
563\end{array}$ \\
\hline MONTH & 648 & 552 & 600 & 670 & 297 & 536 & 706 & 489 & 637 & 710 & 552 & 639 \\
\hline
\end{tabular}


Table 30. Specific conductance for Onion River at Hingham, 1979 and 1980 water years-Continued SPECIFIC CONDUCTANCE (MICROMHOS/CM AT 25 DEG. C), WATER YEAR OCTOBER 1979 TO SEPTEMBER 1980

\begin{tabular}{|c|c|c|c|c|c|c|c|c|c|c|c|c|}
\hline \multirow[t]{2}{*}{ DAY } & $\operatorname{MAX}$ & MIN & MEAN & MAX & MIN & MEAN & $\operatorname{MAX}$ & MIN & MEAN & $\operatorname{MAX}$ & MIN & MEAN \\
\hline & \multicolumn{3}{|c|}{ JUNE } & \multicolumn{3}{|c|}{ JULY } & \multicolumn{3}{|c|}{ AUGUST } & \multicolumn{3}{|c|}{ SEPTEMBER } \\
\hline $\begin{array}{l}1 \\
2 \\
3 \\
4 \\
5\end{array}$ & $\begin{array}{l}574 \\
621 \\
642 \\
628 \\
628\end{array}$ & $\begin{array}{l}545 \\
552 \\
620 \\
598 \\
587\end{array}$ & $\begin{array}{l}553 \\
587 \\
629 \\
612 \\
614\end{array}$ & $\begin{array}{l}469 \\
457 \\
466 \\
453 \\
-\cdots\end{array}$ & $\begin{array}{l}453 \\
446 \\
444 \\
449 \\
---\end{array}$ & $\begin{array}{l}458 \\
451 \\
453 \\
450 \\
-\end{array}$ & $\begin{array}{l}433 \\
441 \\
440 \\
444 \\
454\end{array}$ & $\begin{array}{l}420 \\
430 \\
434 \\
429 \\
428\end{array}$ & $\begin{array}{l}427 \\
436 \\
437 \\
440 \\
442\end{array}$ & $\begin{array}{l}587 \\
592 \\
596 \\
597 \\
602\end{array}$ & $\begin{array}{l}578 \\
552 \\
590 \\
585 \\
565\end{array}$ & $\begin{array}{l}583 \\
583 \\
593 \\
594 \\
585\end{array}$ \\
\hline $\begin{array}{r}6 \\
7 \\
8 \\
9 \\
10\end{array}$ & $\begin{array}{l}614 \\
598 \\
558 \\
479 \\
528\end{array}$ & $\begin{array}{l}586 \\
565 \\
480 \\
466 \\
481\end{array}$ & $\begin{array}{l}595 \\
583 \\
532 \\
471 \\
502\end{array}$ & 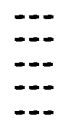 & $\begin{array}{l}\cdots \\
\cdots \\
\cdots \\
\cdots-\end{array}$ & $\begin{array}{l}\cdots \\
\cdots \\
\cdots \\
\cdots \\
\cdots\end{array}$ & $\begin{array}{l}482 \\
476 \\
458 \\
513 \\
506\end{array}$ & $\begin{array}{l}458 \\
448 \\
333 \\
387 \\
488\end{array}$ & $\begin{array}{l}472 \\
459 \\
391 \\
475 \\
494\end{array}$ & $\begin{array}{l}575 \\
583 \\
597 \\
601 \\
594\end{array}$ & $\begin{array}{l}563 \\
565 \\
581 \\
583 \\
581\end{array}$ & $\begin{array}{l}569 \\
574 \\
589 \\
593 \\
586\end{array}$ \\
\hline $\begin{array}{l}11 \\
12 \\
13 \\
14 \\
15\end{array}$ & $\begin{array}{l}554 \\
603 \\
583 \\
604 \\
593\end{array}$ & $\begin{array}{l}499 \\
494 \\
555 \\
565 \\
577\end{array}$ & $\begin{array}{l}525 \\
560 \\
571 \\
585 \\
583\end{array}$ & $\begin{array}{l}\cdots \\
\cdots \\
\cdots \\
\cdots\end{array}$ & $\begin{array}{l}\cdots- \\
\cdots \\
\cdots- \\
\cdots-\end{array}$ & $\begin{array}{l}\cdots \\
\cdots \\
\cdots \\
\cdots\end{array}$ & $\begin{array}{l}518 \\
559 \\
585 \\
612 \\
625\end{array}$ & $\begin{array}{l}497 \\
517 \\
560 \\
589 \\
602\end{array}$ & $\begin{array}{l}502 \\
535 \\
575 \\
600 \\
615\end{array}$ & $\begin{array}{l}592 \\
590 \\
570 \\
572 \\
565\end{array}$ & $\begin{array}{l}576 \\
561 \\
568 \\
564 \\
563\end{array}$ & $\begin{array}{l}583 \\
576 \\
569 \\
567 \\
564\end{array}$ \\
\hline $\begin{array}{l}16 \\
17 \\
18 \\
19 \\
20\end{array}$ & $\begin{array}{l}584 \\
567 \\
539 \\
508 \\
529\end{array}$ & $\begin{array}{l}568 \\
533 \\
508 \\
490 \\
495\end{array}$ & $\begin{array}{l}575 \\
554 \\
523 \\
501 \\
506\end{array}$ & $\begin{array}{l}559 \\
511 \\
510 \\
493 \\
484\end{array}$ & $\begin{array}{l}524 \\
496 \\
494 \\
485 \\
321\end{array}$ & $\begin{array}{l}534 \\
505 \\
501 \\
489 \\
392\end{array}$ & $\begin{array}{l}630 \\
627 \\
621 \\
572 \\
555\end{array}$ & $\begin{array}{l}611 \\
616 \\
534 \\
519 \\
414\end{array}$ & $\begin{array}{l}618 \\
621 \\
592 \\
550 \\
503\end{array}$ & $\begin{array}{l}572 \\
568 \\
586 \\
606 \\
605\end{array}$ & $\begin{array}{l}551 \\
547 \\
554 \\
588 \\
592\end{array}$ & $\begin{array}{l}564 \\
556 \\
568 \\
597 \\
598\end{array}$ \\
\hline $\begin{array}{l}21 \\
22 \\
23 \\
24 \\
25\end{array}$ & $\begin{array}{l}528 \\
516 \\
533 \\
526 \\
514\end{array}$ & $\begin{array}{l}492 \\
495 \\
505 \\
498 \\
493\end{array}$ & $\begin{array}{l}515 \\
508 \\
519 \\
509 \\
506\end{array}$ & $\begin{array}{l}432 \\
415 \\
446 \\
464 \\
492\end{array}$ & $\begin{array}{l}355 \\
395 \\
414 \\
437 \\
462\end{array}$ & $\begin{array}{l}405 \\
405 \\
428 \\
452 \\
477\end{array}$ & $\begin{array}{l}503 \\
510 \\
522 \\
542 \\
549\end{array}$ & $\begin{array}{l}406 \\
497 \\
504 \\
513 \\
525\end{array}$ & $\begin{array}{l}462 \\
503 \\
510 \\
524 \\
541\end{array}$ & $\begin{array}{l}591 \\
592 \\
465 \\
523 \\
553\end{array}$ & $\begin{array}{l}579 \\
311 \\
441 \\
467 \\
511\end{array}$ & $\begin{array}{l}584 \\
436 \\
454 \\
492 \\
529\end{array}$ \\
\hline $\begin{array}{l}26 \\
27 \\
28 \\
29 \\
30 \\
31\end{array}$ & $\begin{array}{l}525 \\
498 \\
495 \\
485 \\
479 \\
-\end{array}$ & $\begin{array}{l}499 \\
490 \\
479 \\
474 \\
457 \\
---\end{array}$ & $\begin{array}{l}511 \\
495 \\
488 \\
478 \\
469 \\
--\end{array}$ & $\begin{array}{l}507 \\
517 \\
534 \\
530 \\
464 \\
468\end{array}$ & $\begin{array}{l}490 \\
500 \\
510 \\
444 \\
441 \\
417\end{array}$ & $\begin{array}{l}501 \\
510 \\
521 \\
495 \\
452 \\
445\end{array}$ & $\begin{array}{l}560 \\
568 \\
560 \\
558 \\
569 \\
579\end{array}$ & $\begin{array}{l}542 \\
558 \\
532 \\
515 \\
553 \\
570\end{array}$ & $\begin{array}{l}554 \\
563 \\
550 \\
536 \\
563 \\
575\end{array}$ & \begin{tabular}{l}
560 \\
\hdashline- \\
\hdashline- \\
\hdashline- \\
-
\end{tabular} & \begin{tabular}{l}
554 \\
$\cdots$ \\
\hdashline- \\
\hdashline- \\
\hdashline-
\end{tabular} & \begin{tabular}{c}
557 \\
0 \\
\hdashline \\
\hdashline
\end{tabular} \\
\hline MONTH & 642 & 457 & 539 & 559 & 321 & 466 & 630 & 333 & 518 & 606 & $3 i 1$ & 563 \\
\hline YEAR & 718 & 297 & 574 & & & & & & & & & \\
\hline
\end{tabular}


Table 31. Specific conductance for Onion River near Sheboygan Falls, 1979 and 1980 water years

SPECIFIC CONDUCTANCE (MICROMHOS/CM AT 25 DEG. C), WATER YEAR OCTOBER 1978 TO SEPTEMBER 1979

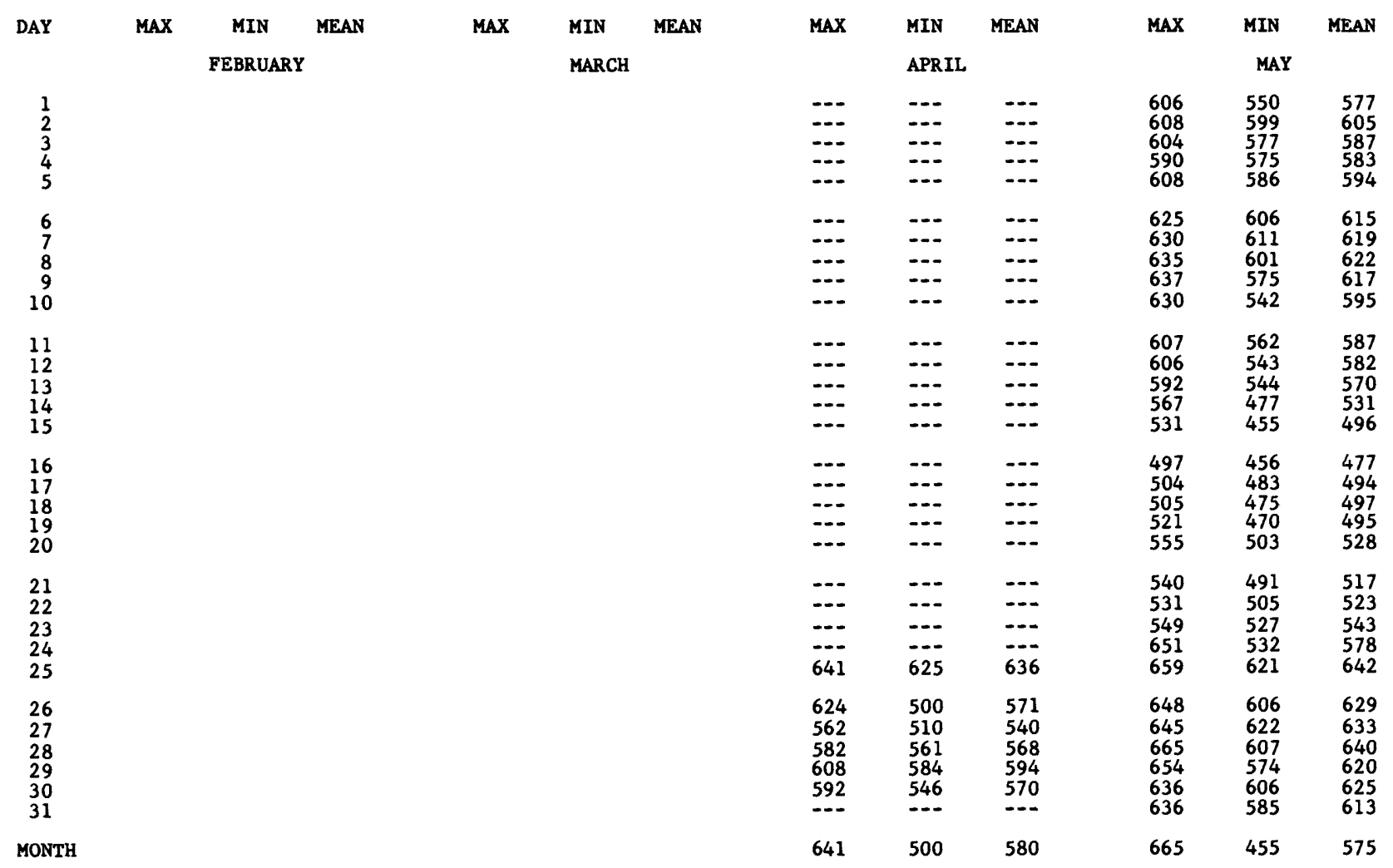

SPECIFIC CONDUCTANCE (MICROMHOS/CM AT 25 DEG. C), WATER YEAR OCTOBER 1978 TO SEPTEMBER 1979

\begin{tabular}{|c|c|c|c|c|c|c|c|c|c|c|c|c|}
\hline \multirow[t]{2}{*}{ DAY } & MAX & MIN & MEAN & MAX & MIN & MEAN & MAX & MIN & MEAN & MAX & MIN & MEAN \\
\hline & \multicolumn{3}{|c|}{ JUNE } & \multicolumn{3}{|c|}{ JULY } & \multicolumn{3}{|c|}{ AUGUST } & \multicolumn{3}{|c|}{ SEPTEMBER } \\
\hline $\begin{array}{l}1 \\
2 \\
3 \\
4 \\
5\end{array}$ & $\begin{array}{l}633 \\
620 \\
627 \\
628 \\
628\end{array}$ & $\begin{array}{l}582 \\
598 \\
599 \\
618 \\
607\end{array}$ & $\begin{array}{l}608 \\
610 \\
616 \\
625 \\
619\end{array}$ & $\begin{array}{l}572 \\
553 \\
542 \\
571 \\
570\end{array}$ & $\begin{array}{l}545 \\
540 \\
524 \\
531 \\
552\end{array}$ & $\begin{array}{l}552 \\
549 \\
534 \\
549 \\
558\end{array}$ & $\begin{array}{l}555 \\
601 \\
601 \\
551 \\
554\end{array}$ & $\begin{array}{l}542 \\
562 \\
554 \\
525 \\
522\end{array}$ & $\begin{array}{l}549 \\
588 \\
582 \\
540 \\
534\end{array}$ & $\begin{array}{l}713 \\
745 \\
763 \\
773 \\
795\end{array}$ & $\begin{array}{l}701 \\
711 \\
748 \\
757 \\
771\end{array}$ & $\begin{array}{l}705 \\
725 \\
757 \\
766 \\
780\end{array}$ \\
\hline $\begin{array}{r}6 \\
7 \\
8 \\
9 \\
10\end{array}$ & $\begin{array}{l}629 \\
634 \\
615 \\
622 \\
626\end{array}$ & $\begin{array}{l}614 \\
602 \\
593 \\
600 \\
601\end{array}$ & $\begin{array}{l}621 \\
619 \\
605 \\
612 \\
612\end{array}$ & $\begin{array}{l}570 \\
551 \\
543 \\
513 \\
537\end{array}$ & $\begin{array}{l}552 \\
533 \\
475 \\
477 \\
498\end{array}$ & $\begin{array}{l}557 \\
543 \\
517 \\
497 \\
526\end{array}$ & $\begin{array}{l}535 \\
609 \\
610 \\
551 \\
464\end{array}$ & $\begin{array}{l}508 \\
537 \\
558 \\
447 \\
390\end{array}$ & $\begin{array}{l}517 \\
568 \\
587 \\
518 \\
426\end{array}$ & $\begin{array}{l}806 \\
841 \\
849 \\
760 \\
739\end{array}$ & $\begin{array}{l}785 \\
813 \\
758 \\
737 \\
728\end{array}$ & $\begin{array}{l}795 \\
832 \\
813 \\
743 \\
732\end{array}$ \\
\hline $\begin{array}{l}11 \\
12 \\
13 \\
14 \\
15\end{array}$ & $\begin{array}{l}620 \\
627 \\
649 \\
650 \\
654\end{array}$ & $\begin{array}{l}605 \\
618 \\
628 \\
642 \\
643\end{array}$ & $\begin{array}{l}613 \\
623 \\
639 \\
648 \\
650\end{array}$ & $\begin{array}{l}534 \\
531 \\
526 \\
519 \\
497\end{array}$ & $\begin{array}{l}526 \\
513 \\
512 \\
493 \\
488\end{array}$ & $\begin{array}{l}532 \\
525 \\
520 \\
506 \\
492\end{array}$ & $\begin{array}{l}466 \\
486 \\
525 \\
545 \\
563\end{array}$ & $\begin{array}{l}397 \\
468 \\
490 \\
527 \\
542\end{array}$ & $\begin{array}{l}432 \\
474 \\
508 \\
535 \\
553\end{array}$ & $\begin{array}{l}730 \\
705 \\
712 \\
692 \\
697\end{array}$ & $\begin{array}{l}703 \\
656 \\
631 \\
680 \\
681\end{array}$ & $\begin{array}{l}717 \\
686 \\
667 \\
685 \\
690\end{array}$ \\
\hline $\begin{array}{l}16 \\
17 \\
18 \\
19 \\
20\end{array}$ & $\begin{array}{l}651 \\
644 \\
637 \\
646 \\
623\end{array}$ & $\begin{array}{l}630 \\
634 \\
594 \\
592 \\
598\end{array}$ & $\begin{array}{l}644 \\
639 \\
620 \\
618 \\
609\end{array}$ & $\begin{array}{l}505 \\
505 \\
500 \\
504 \\
508\end{array}$ & $\begin{array}{l}492 \\
487 \\
481 \\
476 \\
495\end{array}$ & $\begin{array}{l}499 \\
499 \\
490 \\
489 \\
501\end{array}$ & $\begin{array}{l}580 \\
588 \\
612 \\
670 \\
703\end{array}$ & $\begin{array}{l}558 \\
582 \\
591 \\
614 \\
676\end{array}$ & $\begin{array}{l}568 \\
585 \\
601 \\
637 \\
689\end{array}$ & $\begin{array}{l}698 \\
718 \\
708 \\
687 \\
565\end{array}$ & $\begin{array}{l}683 \\
703 \\
681 \\
664 \\
614\end{array}$ & $\begin{array}{l}691 \\
711 \\
698 \\
678 \\
650\end{array}$ \\
\hline $\begin{array}{l}21 \\
22 \\
23 \\
24 \\
25\end{array}$ & $\begin{array}{l}636 \\
656 \\
660 \\
657 \\
646\end{array}$ & $\begin{array}{l}599 \\
634 \\
645 \\
645 \\
586\end{array}$ & $\begin{array}{l}617 \\
645 \\
654 \\
652 \\
622\end{array}$ & $\begin{array}{l}517 \\
552 \\
586 \\
585 \\
591\end{array}$ & $\begin{array}{l}493 \\
518 \\
549 \\
557 \\
557\end{array}$ & $\begin{array}{l}506 \\
541 \\
561 \\
572 \\
578\end{array}$ & $\begin{array}{l}705 \\
624 \\
635 \\
588 \\
563\end{array}$ & $\begin{array}{l}616 \\
615 \\
595 \\
489 \\
494\end{array}$ & $\begin{array}{l}632 \\
619 \\
624 \\
537 \\
530\end{array}$ & $\begin{array}{l}618 \\
610 \\
602 \\
607 \\
617\end{array}$ & $\begin{array}{l}587 \\
582 \\
576 \\
575 \\
596\end{array}$ & $\begin{array}{l}607 \\
595 \\
590 \\
595 \\
606\end{array}$ \\
\hline $\begin{array}{l}26 \\
27 \\
28 \\
29 \\
30 \\
31\end{array}$ & $\begin{array}{l}587 \\
606 \\
608 \\
589 \\
592 \\
-\end{array}$ & $\begin{array}{l}560 \\
578 \\
572 \\
512 \\
527 \\
-\end{array}$ & $\begin{array}{l}576 \\
596 \\
595 \\
557 \\
559 \\
---\end{array}$ & $\begin{array}{l}603 \\
581 \\
587 \\
569 \\
558 \\
552\end{array}$ & $\begin{array}{l}572 \\
567 \\
567 \\
553 \\
537 \\
529\end{array}$ & $\begin{array}{l}593 \\
574 \\
575 \\
564 \\
546 \\
542\end{array}$ & $\begin{array}{l}592 \\
610 \\
627 \\
636 \\
685 \\
703\end{array}$ & $\begin{array}{l}568 \\
594 \\
612 \\
623 \\
631 \\
691\end{array}$ & $\begin{array}{l}581 \\
603 \\
618 \\
629 \\
648 \\
696\end{array}$ & $\begin{array}{l}597 \\
581 \\
628 \\
746 \\
779 \\
---\end{array}$ & $\begin{array}{l}556 \\
555 \\
569 \\
632 \\
747 \\
---\end{array}$ & $\begin{array}{l}584 \\
568 \\
596 \\
691 \\
762 \\
-\end{array}$ \\
\hline MONTH & 660 & 512 & 617 & 603 & 475 & 535 & 705 & 390 & 571 & 849 & 555 & 691 \\
\hline
\end{tabular}

YEAR $\quad 849 \quad 390 \quad 597$

NOTE: NUMBER OF MISSING DAYS OF RECORD EXCEEDED 20\% OF YEAR 
Table 31. Specific conductance for Onion River near Sheboygan Falls, 1979 and 1980 water years--Continued SPECIFIC CONDUCTANCE (MICROMHOS/CM AT 25 DEG. C), WATER YEAR OCTOBER 1979 TO SEPTEMBER 1980

\begin{tabular}{|c|c|c|c|c|c|c|c|c|c|c|c|c|}
\hline \multirow[t]{2}{*}{ DAY } & $\operatorname{MAX}$ & MIN & MEAN & $\operatorname{MAX}$ & MIN & MEAN & $\operatorname{MAX}$ & MIN & MEAN & $\operatorname{MAX}$ & MIN & MEAN \\
\hline & \multicolumn{3}{|c|}{ OCTOBER } & \multicolumn{3}{|c|}{ NOVEMBER } & \multicolumn{3}{|c|}{ DECEMBER } & \multicolumn{3}{|c|}{ JANUARY } \\
\hline $\begin{array}{l}1 \\
2 \\
3 \\
4 \\
5\end{array}$ & $\begin{array}{l}776 \\
738 \\
716 \\
702 \\
692\end{array}$ & $\begin{array}{l}737 \\
715 \\
701 \\
691 \\
669\end{array}$ & $\begin{array}{l}756 \\
730 \\
711 \\
697 \\
683\end{array}$ & $\begin{array}{l}798 \\
830 \\
812 \\
802 \\
819\end{array}$ & $\begin{array}{l}706 \\
803 \\
785 \\
775 \\
807\end{array}$ & $\begin{array}{l}741 \\
820 \\
804 \\
787 \\
813\end{array}$ & $\begin{array}{l}717 \\
732 \\
746 \\
761 \\
744\end{array}$ & $\begin{array}{l}702 \\
720 \\
735 \\
741 \\
698\end{array}$ & $\begin{array}{l}709 \\
728 \\
743 \\
750 \\
719\end{array}$ & $\begin{array}{l}709 \\
707 \\
734 \\
730 \\
742\end{array}$ & $\begin{array}{l}690 \\
699 \\
703 \\
715 \\
722\end{array}$ & $\begin{array}{l}700 \\
702 \\
717 \\
723 \\
729\end{array}$ \\
\hline $\begin{array}{r}6 \\
7 \\
8 \\
9 \\
10\end{array}$ & $\begin{array}{l}670 \\
658 \\
650 \\
654 \\
655\end{array}$ & $\begin{array}{l}650 \\
648 \\
631 \\
632 \\
643\end{array}$ & $\begin{array}{l}662 \\
653 \\
642 \\
644 \\
648\end{array}$ & $\begin{array}{l}821 \\
810 \\
807 \\
785 \\
806\end{array}$ & $\begin{array}{l}808 \\
779 \\
780 \\
762 \\
775\end{array}$ & $\begin{array}{l}812 \\
791 \\
795 \\
771 \\
790\end{array}$ & $\begin{array}{l}705 \\
690 \\
715 \\
744 \\
759\end{array}$ & $\begin{array}{l}653 \\
649 \\
655 \\
710 \\
689\end{array}$ & $\begin{array}{l}687 \\
677 \\
690 \\
725 \\
724\end{array}$ & $\begin{array}{l}742 \\
804 \\
861 \\
873 \\
895\end{array}$ & $\begin{array}{l}734 \\
730 \\
805 \\
853 \\
825\end{array}$ & $\begin{array}{l}739 \\
762 \\
831 \\
863 \\
875\end{array}$ \\
\hline $\begin{array}{l}11 \\
12 \\
13 \\
14 \\
15\end{array}$ & $\begin{array}{l}650 \\
648 \\
650 \\
641 \\
640\end{array}$ & $\begin{array}{l}644 \\
648 \\
643 \\
632 \\
630\end{array}$ & $\begin{array}{l}647 \\
648 \\
646 \\
637 \\
635\end{array}$ & $\begin{array}{l}818 \\
--- \\
--- \\
\overline{865}\end{array}$ & $\begin{array}{c}807 \\
--- \\
- \\
830\end{array}$ & \begin{tabular}{c}
813 \\
\hdashline-- \\
859
\end{tabular} & $\begin{array}{l}704 \\
701 \\
707 \\
723 \\
679\end{array}$ & $\begin{array}{l}664 \\
651 \\
648 \\
609 \\
648\end{array}$ & $\begin{array}{l}685 \\
685 \\
667 \\
678 \\
668\end{array}$ & $\begin{array}{l}817 \\
789 \\
821 \\
823 \\
708\end{array}$ & $\begin{array}{l}739 \\
747 \\
797 \\
751 \\
682\end{array}$ & $\begin{array}{l}765 \\
767 \\
809 \\
784 \\
700\end{array}$ \\
\hline $\begin{array}{l}16 \\
17 \\
18 \\
19 \\
20\end{array}$ & $\begin{array}{l}647 \\
658 \\
677 \\
683 \\
646\end{array}$ & $\begin{array}{l}641 \\
645 \\
659 \\
648 \\
630\end{array}$ & $\begin{array}{l}644 \\
650 \\
668 \\
670 \\
637\end{array}$ & $\begin{array}{l}841 \\
838 \\
841 \\
818 \\
811\end{array}$ & $\begin{array}{l}815 \\
823 \\
810 \\
798 \\
768\end{array}$ & $\begin{array}{l}827 \\
827 \\
823 \\
808 \\
788\end{array}$ & $\begin{array}{l}691 \\
722 \\
780 \\
790 \\
715\end{array}$ & $\begin{array}{l}660 \\
695 \\
726 \\
718 \\
649\end{array}$ & $\begin{array}{l}678 \\
707 \\
754 \\
750 \\
676\end{array}$ & $\begin{array}{l}681 \\
702 \\
589 \\
588 \\
639\end{array}$ & $\begin{array}{l}444 \\
577 \\
563 \\
572 \\
598\end{array}$ & $\begin{array}{l}660 \\
611 \\
573 \\
578 \\
619\end{array}$ \\
\hline $\begin{array}{l}21 \\
22 \\
23 \\
24 \\
25\end{array}$ & $\begin{array}{l}681 \\
694 \\
695 \\
735 \\
705\end{array}$ & $\begin{array}{l}641 \\
679 \\
671 \\
672 \\
640\end{array}$ & $\begin{array}{l}656 \\
686 \\
680 \\
705 \\
654\end{array}$ & $\begin{array}{l}785 \\
822 \\
830 \\
713 \\
689\end{array}$ & $\begin{array}{l}754 \\
764 \\
687 \\
682 \\
673\end{array}$ & $\begin{array}{l}767 \\
780 \\
726 \\
697 \\
686\end{array}$ & $\begin{array}{l}673 \\
655 \\
628 \\
599 \\
590\end{array}$ & $\begin{array}{l}653 \\
628 \\
602 \\
538 \\
520\end{array}$ & $\begin{array}{l}663 \\
639 \\
614 \\
561 \\
552\end{array}$ & $\begin{array}{l}689 \\
718 \\
764 \\
812 \\
814\end{array}$ & $\begin{array}{l}650 \\
695 \\
726 \\
775 \\
804\end{array}$ & $\begin{array}{l}675 \\
710 \\
747 \\
792 \\
809\end{array}$ \\
\hline $\begin{array}{l}26 \\
27 \\
28 \\
29 \\
30 \\
31\end{array}$ & $\begin{array}{l}675 \\
708 \\
736 \\
749 \\
769 \\
751\end{array}$ & $\begin{array}{l}647 \\
676 \\
709 \\
733 \\
750 \\
701\end{array}$ & $\begin{array}{l}659 \\
693 \\
725 \\
739 \\
758 \\
721\end{array}$ & $\begin{array}{l}676 \\
721 \\
697 \\
677 \\
718 \\
---\end{array}$ & $\begin{array}{l}658 \\
679 \\
670 \\
670 \\
680 \\
---\end{array}$ & $\begin{array}{l}667 \\
701 \\
678 \\
672 \\
702 \\
---\end{array}$ & $\begin{array}{l}596 \\
646 \\
663 \\
678 \\
705 \\
721\end{array}$ & $\begin{array}{l}549 \\
607 \\
625 \\
644 \\
667 \\
683\end{array}$ & $\begin{array}{l}575 \\
620 \\
639 \\
662 \\
687 \\
699\end{array}$ & $\begin{array}{l}807 \\
809 \\
815 \\
827 \\
833 \\
832\end{array}$ & $\begin{array}{l}794 \\
794 \\
808 \\
815 \\
825 \\
821\end{array}$ & $\begin{array}{l}799 \\
803 \\
812 \\
822 \\
828 \\
825\end{array}$ \\
\hline MONTH & 776 & 630 & 677 & 865 & 658 & 768 & 790 & 520 & 678 & 895 & 444 & 746 \\
\hline
\end{tabular}

SPECIFIC CONDUCTANCE (MICROMHOS/CM AT 25 DEg. C), WATER YEAR OCTOBER 1979 TO SEPTEMBER 1980

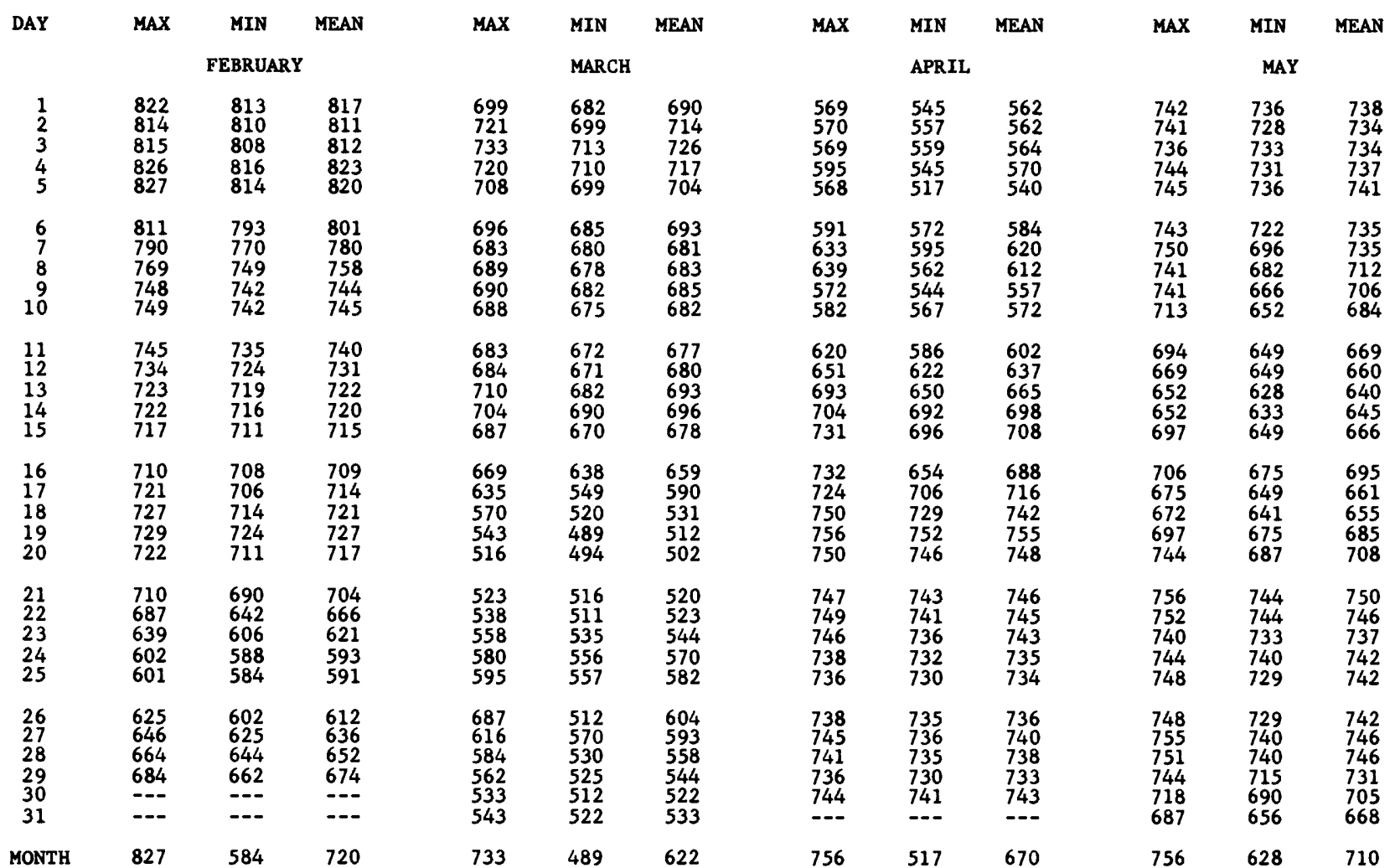


Table 31. Specific conductance for Onion River near Sheboygan Falls, 1979 and 1980 water years--Continued SPECIFIC CONDUCTANCE (MICROMHOS/CM AT 25 DEG. C), WATER YEAR OCTOBER 1979 TO SEPTEMBER 1980

\begin{tabular}{|c|c|c|c|c|c|c|c|c|c|c|c|c|}
\hline \multirow[t]{2}{*}{ DAY } & $\operatorname{MAX}$ & MIN & MEAN & MAX & MIN & MEAN & MAX & MIN & MEAN & $\max$ & MIN & MEAN \\
\hline & \multicolumn{3}{|c|}{ JUNE } & \multicolumn{3}{|c|}{ JULY } & \multicolumn{3}{|c|}{ AUGUST } & \multicolumn{3}{|c|}{ SEPTEMBER } \\
\hline $\begin{array}{l}1 \\
2 \\
3 \\
4 \\
5\end{array}$ & $\begin{array}{l}674 \\
665 \\
674 \\
671 \\
674\end{array}$ & $\begin{array}{l}662 \\
656 \\
656 \\
665 \\
645\end{array}$ & $\begin{array}{l}669 \\
661 \\
664 \\
668 \\
659\end{array}$ & $\begin{array}{l}724 \\
712 \\
690 \\
627 \\
713\end{array}$ & $\begin{array}{l}697 \\
639 \\
590 \\
603 \\
621\end{array}$ & $\begin{array}{l}715 \\
682 \\
641 \\
616 \\
684\end{array}$ & $\begin{array}{l}577 \\
596 \\
597 \\
590 \\
607\end{array}$ & $\begin{array}{l}557 \\
567 \\
581 \\
580 \\
580\end{array}$ & $\begin{array}{l}565 \\
587 \\
589 \\
585 \\
595\end{array}$ & $\begin{array}{l}808 \\
798 \\
795 \\
855 \\
832\end{array}$ & $\begin{array}{l}789 \\
755 \\
733 \\
784 \\
792\end{array}$ & $\begin{array}{l}800 \\
779 \\
754 \\
817 \\
817\end{array}$ \\
\hline $\begin{array}{r}6 \\
7 \\
8 \\
9 \\
10\end{array}$ & $\begin{array}{l}650 \\
677 \\
671 \\
650 \\
621\end{array}$ & $\begin{array}{l}632 \\
624 \\
653 \\
540 \\
525\end{array}$ & $\begin{array}{l}642 \\
657 \\
661 \\
611 \\
563\end{array}$ & $\begin{array}{l}709 \\
774 \\
755 \\
732 \\
756\end{array}$ & $\begin{array}{l}682 \\
709 \\
671 \\
706 \\
725\end{array}$ & $\begin{array}{l}698 \\
741 \\
695 \\
723 \\
741\end{array}$ & $\begin{array}{l}592 \\
647 \\
615 \\
534 \\
611\end{array}$ & $\begin{array}{l}573 \\
587 \\
513 \\
487 \\
537\end{array}$ & $\begin{array}{l}584 \\
617 \\
561 \\
502 \\
579\end{array}$ & $\begin{array}{l}843 \\
853 \\
868 \\
879 \\
846\end{array}$ & $\begin{array}{l}801 \\
821 \\
799 \\
831 \\
800\end{array}$ & $\begin{array}{l}826 \\
838 \\
824 \\
863 \\
821\end{array}$ \\
\hline $\begin{array}{l}11 \\
12 \\
13 \\
14 \\
15\end{array}$ & $\begin{array}{l}642 \\
624 \\
714 \\
733 \\
726\end{array}$ & $\begin{array}{l}624 \\
616 \\
643 \\
710 \\
707\end{array}$ & $\begin{array}{l}632 \\
620 \\
672 \\
722 \\
720\end{array}$ & $\begin{array}{l}744 \\
741 \\
675 \\
647 \\
641\end{array}$ & $\begin{array}{l}721 \\
679 \\
647 \\
638 \\
602\end{array}$ & $\begin{array}{l}734 \\
718 \\
665 \\
643 \\
627\end{array}$ & $\begin{array}{l}650 \\
707 \\
727 \\
751 \\
779\end{array}$ & $\begin{array}{l}612 \\
654 \\
708 \\
724 \\
752\end{array}$ & $\begin{array}{l}627 \\
683 \\
715 \\
737 \\
765\end{array}$ & $\begin{array}{l}847 \\
825 \\
844 \\
831 \\
728\end{array}$ & $\begin{array}{l}797 \\
794 \\
814 \\
727 \\
704\end{array}$ & $\begin{array}{l}822 \\
806 \\
829 \\
774 \\
711\end{array}$ \\
\hline $\begin{array}{l}16 \\
17 \\
18 \\
19 \\
20\end{array}$ & $\begin{array}{l}726 \\
722 \\
722 \\
711 \\
692\end{array}$ & $\begin{array}{l}707 \\
695 \\
707 \\
680 \\
632\end{array}$ & $\begin{array}{l}716 \\
709 \\
716 \\
690 \\
672\end{array}$ & $\begin{array}{l}602 \\
638 \\
652 \\
628 \\
610\end{array}$ & $\begin{array}{l}578 \\
570 \\
605 \\
609 \\
454\end{array}$ & $\begin{array}{l}586 \\
585 \\
622 \\
619 \\
512\end{array}$ & $\begin{array}{l}787 \\
796 \\
797 \\
786 \\
794\end{array}$ & $\begin{array}{l}772 \\
780 \\
774 \\
763 \\
552\end{array}$ & $\begin{array}{l}778 \\
789 \\
785 \\
773 \\
694\end{array}$ & $\begin{array}{l}711 \\
734 \\
788 \\
793 \\
790\end{array}$ & $\begin{array}{l}677 \\
687 \\
723 \\
785 \\
767\end{array}$ & $\begin{array}{l}699 \\
709 \\
756 \\
790 \\
777\end{array}$ \\
\hline $\begin{array}{l}21 \\
22 \\
23 \\
24 \\
25\end{array}$ & $\begin{array}{l}638 \\
613 \\
626 \\
645 \\
654\end{array}$ & $\begin{array}{l}596 \\
581 \\
573 \\
607 \\
617\end{array}$ & $\begin{array}{l}619 \\
596 \\
597 \\
636 \\
639\end{array}$ & $\begin{array}{l}535 \\
585 \\
601 \\
616 \\
611\end{array}$ & $\begin{array}{l}453 \\
536 \\
575 \\
602 \\
603\end{array}$ & $\begin{array}{l}498 \\
569 \\
589 \\
608 \\
607\end{array}$ & $\begin{array}{l}637 \\
650 \\
728 \\
805 \\
814\end{array}$ & $\begin{array}{l}555 \\
618 \\
648 \\
756 \\
787\end{array}$ & $\begin{array}{l}601 \\
633 \\
684 \\
787 \\
802\end{array}$ & $\begin{array}{l}779 \\
768 \\
448 \\
491 \\
551\end{array}$ & $\begin{array}{l}722 \\
417 \\
423 \\
451 \\
495\end{array}$ & $\begin{array}{l}750 \\
520 \\
436 \\
472 \\
519\end{array}$ \\
\hline $\begin{array}{l}26 \\
27 \\
28 \\
29 \\
30 \\
31\end{array}$ & $\begin{array}{l}689 \\
723 \\
739 \\
747 \\
813 \\
---\end{array}$ & $\begin{array}{l}645 \\
685 \\
667 \\
667 \\
724 \\
---\end{array}$ & $\begin{array}{l}676 \\
709 \\
709 \\
711 \\
765 \\
---\end{array}$ & $\begin{array}{l}612 \\
619 \\
647 \\
651 \\
640 \\
611\end{array}$ & $\begin{array}{l}601 \\
605 \\
620 \\
636 \\
610 \\
559\end{array}$ & $\begin{array}{l}605 \\
611 \\
636 \\
646 \\
631 \\
596\end{array}$ & $\begin{array}{l}862 \\
825 \\
782 \\
767 \\
772 \\
807\end{array}$ & $\begin{array}{l}815 \\
773 \\
755 \\
710 \\
741 \\
773\end{array}$ & $\begin{array}{l}836 \\
806 \\
769 \\
743 \\
758 \\
794\end{array}$ & $\begin{array}{l}624 \\
665 \\
696 \\
713 \\
729 \\
--\end{array}$ & $\begin{array}{l}557 \\
627 \\
665 \\
697 \\
710 \\
---\end{array}$ & $\begin{array}{l}594 \\
650 \\
682 \\
706 \\
721 \\
---\end{array}$ \\
\hline MONTH & 813 & 525 & 666 & 774 & 453 & 640 & 862 & 487 & 688 & 879 & 417 & 729 \\
\hline YEAR & 895 & 417 & 692 & & & & & & & & & \\
\hline
\end{tabular}

